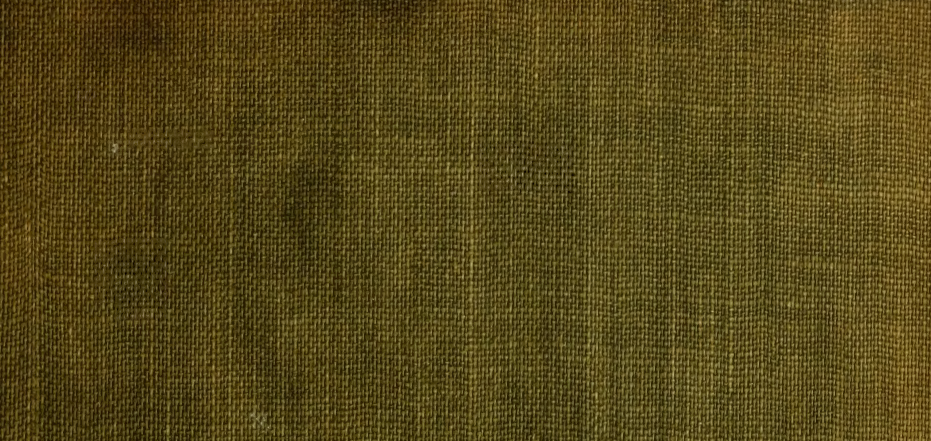




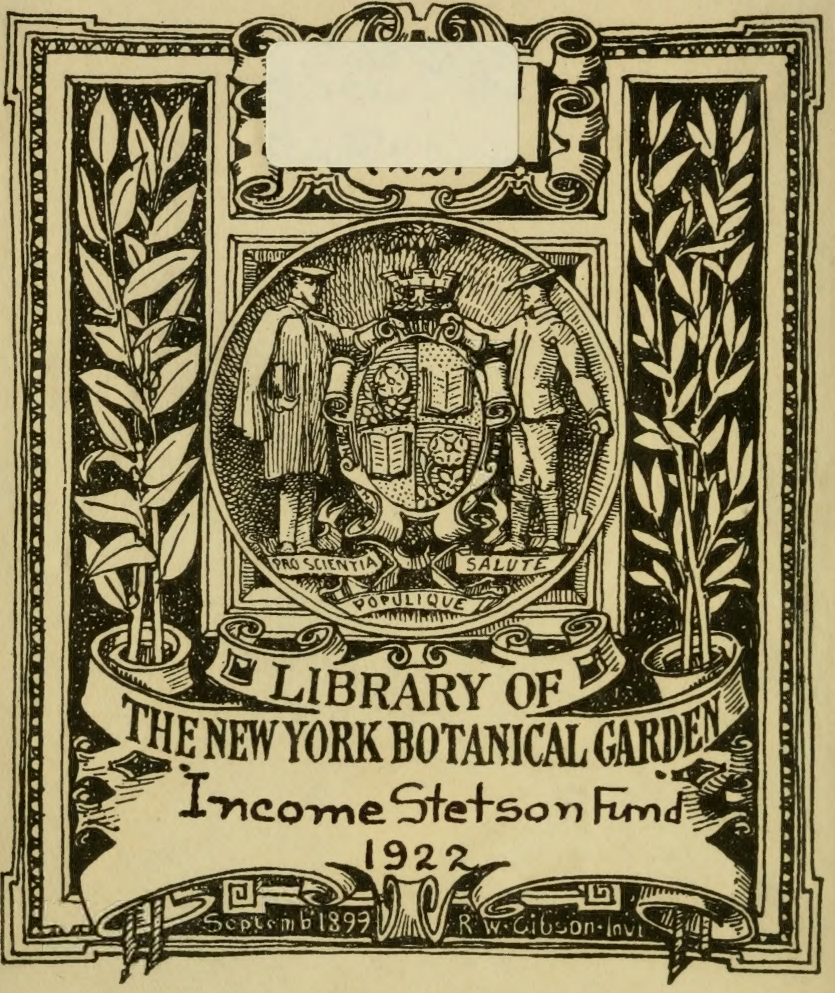






\section{A}

FLORA SEGUSINA

DI G. FRANGESCO RE

RIPRODOTTA NEL MRTODO NATURALE DI DE CANDOLLE

E CONENTATA

\section{DA BENIAMINO CASO}

PUBBLICATA

PER INIZIATIVA E CURA DELLA SEZIONE DI SUSA

del Club Alpino Italiano

PRECEDUTA DALLA VITA DELL'AUTORE

scritta dal Prof. M. Lessona

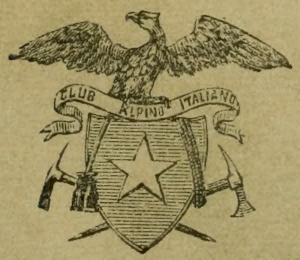

TORINO

A ХGLO BAGLIONE, TIPOG'RAFO

Via Bogino, Numero 23

1881 


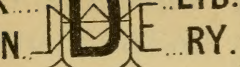




\title{
L A
}

\section{FLORA \\ SEGUSINA}

\section{G. FRANGESCO RE}

RIPRODOTTA NEL METODO NATURALE DI DR CANDOLIE

E COMENTATA

\section{DA BENIAIMINO GASO}

\author{
PUBBLICATA
}

PER INIZIATIVA E CURA DELLA SEZIONE DI SUSA

del Club lpino Italiano

PRECEDUTA DALLA VITA DELL' AUTORE

seritta dal Prof. M. Lessona

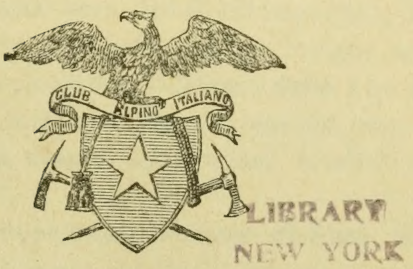

BUTANCAAL

TORINO

ANgelo BaglioNe, TIPOGRAFO

Via Bogino, Numero 23

$1 \overline{881}$ 
$Q K 332$

I R 4

1881 


\section{LIER A AR W \\ NEV \\ BOT \\ GAKLAN \\ xh TRADURTORB}

al Benevolo Lettore

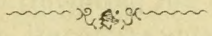

La Sezione di Susa del Club Alpino Italiano, alla quale presiede un uomo non comune per doti egregie di mente e di cuore, il cav. Felice Chiapusso, volle affidarci l'onorevole incarico di tradurre dal latino nella nostra lingua un libro pregevolissimo, scritto nel primo quinquennio del nostro secolo e diventato in oggi una vera rarità bibliografica, la Floru Segusiensis del dottore G. Francesco Re, insigne scienziato nato a Condove ed esploratore indefesso delle Alpi in tempi nei quali l'alpinismo non era entrato ancora, non diremo nei costumi, ma nella mente della quasi totalità dei suoi contemporanei.

Ed ecco come la benemerita Sezione di Susa in tutti i suoi atti manifesta di avere appieno compreso lo scopo altissimo a cui deve tendere la sua nobile instituzione. Essa, dopo di avere vendicato della ingiura di un obblio ingrato i nomi di Colombano Romean e di G. F. Francesco Medail, rende con la presente pubblicazione non solo un omaggio alla memoria di un suo illustre conterraneo, ma ben anche un servizio ai cultori della più bella tra le umane discipline, la Botanica. 
Chi sia stato il dottor G. Francesco Re dirà più appresso una penna illustre, alla quale dobbiamo esprimere tutta la nostra riconoscenza, per non aver disdegnato d'intervenire in queste pagine.

Ed ora una parola sul traduttore e sulla traduzione del volume del dottore condoviano.

Confessiamo francamente innanzi tutto che l'ambizione di rendere un servizio agli amici e colleghi carissimi della Sezione di Susa ci velò, forse per la prima volta in nostra vita, la coscienza che abbiamo sempre avuta della nostra pochezza, e ci dette la forza di vincere la ritrosia di presentarci al pubblico da noi parimenti costantemente sentita; epperò, a scanso di apprezzamenti inesatti sul valore del nostro lavoro, noi dichiariamo di non aver fatto altro che riprodurre fedelmente la Flora Segusina del Re, apportandovi, nella misura delle nostre povere forze, e con la speranza che altri, spronato da questa, faccia cosa più completa, alcune modificazioni nella forma ed ampliandola con annotazioni.

Noi abbiamo trasportato la Flora Segusina dal sistema artificiale di Linneo, in cui fu scritta, nel metodo naturale di De Candolle, il quale, se non è il più razionale, è sicuramente il più pratico; ed abbiamo aggruppato di qua e di là i generi e le specie sotto un carattere comune, perchè la loro determinazione si rendesse men difficile in campagna, specialmente ai giovani non ancora del tutto diventati familiari con la Fitografia. Notammo l'epoca della fioritura delle specie, e, per soddisfare ad un desiderio dei nostri amici di Susa, il nome italiano e francese delle medesime. Qui ci sia concesso di aprire una parentesi, con l'osservare che i nomi italiani delle specie spontanee, che noi togliemmo dal Bertoloni, sono in massima parte barocchi, taluni addirittura indecenti, poco, anzi niente corrispondenti al nome latino ed ai caratteri delle piante. Speriamo ch'essi vengano riformati col progresso degli studi botanici in Italia.

Abbiamo rettificato la nomenclatura, in molte parti poco esatta, segnatamente per le specie supposte di Linneo dal- 
l'autore. E se qualche volta abbiamo errato, il benevolo lettore ricordi che l'erbario del Re trovasi nell' Università di Sassari e a noi non fu dato di riscontrarlo.

Parecchie piante sono comparse nel territorio Segusino dopo la pubblicazione della Flora; parecchie esistenti a quell'epoca ed in oggi, furono dimenticate dall'autore; alcune altre sono scomparse (1). Noi abbiamo aggiunto le prime e le seconde, e per fermo non son tutte, desumendole da fonti sicure, e notandole con un asterisco; abbiamo fatto cenno altresi delle specie che, per quanto abbiamo potuto indagare, non furono più rinvenute, nonchè delle principali coltivate. Abbiamo in ultimo spesse volte citato $\mathrm{i}$ nomi dei botanici posteriori al Re sia per aver essi rinvenuto le piante nei siti indicati dall'autore, sia per averle raccolte in altri luoghi. Le località non seguite da un nome in parentesi sono nostre.

E qui ci corre l'obbligo strettissimo di ringraziare vivamente tutti coloro che ci vollero prestare il loro valido concol'so: l'Arcangeli, direttore dell' Orto botanico torinese; il nostro pregiato e caro amico prof. Bruno, di lui primo assistente, uomo tanto modesto quanto intelligente della materia; il giardiniere dell' Orto medesimo, signor De Filippi, esperto e antico esploratore della Flora piemontese. Manifestiamo parimenti i nostri caldi ringraziamenti al gentile libraio cav. F. Casanova che ci fece tenere un prezioso Elenco delle principali piante spontance crescenti nei dintorni di Susa e sul Cenisio, del dotto e diligentissimo botanico

(1) È risaputo che una Flura locale è stabile a condizione che invariati rimangano il suolo e l'ambiente nel quale essa è nata, locchè è assai difficile a verificarsi per tutta la sua estensione, e specialmente in prossimità delle stazioni dell'uomo modificatore potentissimo della superficie terrestre e dei climi.

Leggasi a questo proposito la stupenda prolusione agli appunti botanici del ch. prof. A. Goiran nella Cronaca alpina 1878-79 della sezione Veronese del Club Alpino Italiano, pag. 59 (Verona, 1880, per Gius. Civelli). 
avv. Francesco Negri di Casale, della di cui amicizia noi ci sentiamo altamente onorati, elenco che, destinato ad essere pubblicato nella Guida al traforo del Frejus, rimase, non sappiamo per quali ragioni, inedito. Ringraziamo in ultimo la signora Gras che ci concedette di riscontrare i manoscritti e le note botaniche lasciate dal defunto di lei marito, Augusto Gras, troppo presto rapito alla scienza.

Ma fra tutto cio che noi abbiamo potuto, in gran parte da semplici e deboli compilatori, eseguire in aggiunta alla Flora Segusina, ed una personale, accurata revisione della vegetazione della valle di Susa, fatta con tutti quei criterii che la scienza oggi reclama, corre gran differenza. Questa sarà opera dell avvenire e di polsi assai più robusti dei nostri. Noi siamo paghi di avere posto una pietra nelle fondamenta del futuro edifizio, e la Sezione di Susa si è resa meritevole della gratitudine dei giovani studiosi di botanica. i quali troveranno nel facile acquisto del libro di G. Francesco Re più agevole la via ad investigazioni più accurate.

Ci rimane a dire un' altra parola. Il Re, dottore in medicina, dava alla sua Flora un'impronta medica, notando tutte le virtu medicinali delle piante; noi, senza entrare in ardue discussioni di materia medica, abbiamo notato in molti punti ciò che vi è di vero o di esagerato nelle asserzioni dell'autore a questo riguardo.

Ed ora non ci resta che ad implorare benigno il giudizio dei nostri lettori.

Torino, Settembre 1881.

Beniamino Caso. Socio della Sezione di Napoli del Club Alpino Italiano

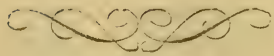




\section{GIOVANNI FRANCESCOO RE}

In sul finire dello scorso secolo lo Allioni diede un impulso poderosissimo in Piemonte agli studi della botanica, la quale scienza, del resto, in questa provincia era a un dipresso la sola fra le scienze naturali che avesse culto intenso ed esteso. Dico a un dipresso, perchè in quel tratto di tempo furono fatti alcuni studi intorno alla mineralogia del Piemonte. Della geologia non si era guari parlato, e la zoologia doveva sorgere e grandeggiare, con Franco Andrea Bonelli, solo nel principio di questo secolo.

Lo Allioni, come avviene sovente di un uomo che veramente ami la scienza sua, come avvenne al tempo nostro in Genova al De Notaris, giovò alla botanica in due modi: colle sue proprie ricerche, coi suoi studi e colle sue pubblicazioni, e in secondo luog'o coll'invogliare altri di questi studi e suscitare alle investigazioni, segnatamente gli scolari. 
Nella schiera dei botanici valenti che diedero opera agli studi intorno alla bella scienza in Piemonte dopo l'Allioni ha un posto segnalato Gio. vanni Francesco Re, di cui la memoria vive fra gli studiosi e si apprezzano oggi i meriti e si ricercano grli șcritti, come appunto avviene di chi ha fatto qualche cosa di veramente utile, per cui vive nel tempo.

Nella bellissima valle di Susa, dove veramente sono oltremodo favorevoli le condizioni per lo studio delle piante, nacque Giovanni Francesco Re. Il suo nascimento segui il giorno 27 di settembre dell'anno 1772. Suo padre si chiamava Giovanni Battista, sua madre Lucia Franzone. Egli nacque in Condove, piccolo villaggio sulla riva sinistra della Dora, a mezzo della valle, quasi in faccia alla pittoresca e storica. Sacra di S. Michele.

Il padre del Re, da quanto pare, possedeva qualche po' di terra e se la coltivava, era quello che allora si chiamava, e anche ogrgi in Piemonte si chiama, un particolare. Non si sa, o almeno, per quanta cura io ci abbia posto, non ho potuto sapere, dove Giovanni Francesco Re facesse i primi studi. Tutto quello che allora fosse possibile imparare a Condove era l'alfabeto, e ciò ancora non per cura del Municipio o del Governo. Nei paesetti come Condove i particoluri che avevano figli, ed erano generalmente molto prolifici, si associavano, mettendo insieme colla spesa in comune una somma che variava fra le 50 e le 200 lire all'anno, face- 
vano venire un prete a insegnare ai loro figliuoli. Qualche volta il prete maestro si allogava dal parroco cui dava aiuto nel confessare e nelle altre operazioni della chiesa, aveva le messe che allora costituivano una rendita certa, era invitato frequentemente a pranzo nelle famiglie dei suoi scolari. Questi scolari portavano ciascheduno alla scuola durante tutto l'inverno un pezzo di legno ogni mattina. Tutti questi pezzi di legno, numerosi pel numero degli scolari, servivano non solo pel riscaldamento della scrola, ma auche per la cucina e per la fiammata serale del maestro. Quest'uso si protrasse abbastanza avanti anche nel secolo corrente e io, che scrivo queste linee, ho il rimorso di avere, nel mio nativo paesetto, dato un mattino, in una contestazione al momento d' entrare in scuola, il mio legrno sulla testa a un compagno. - Fra i vantaggi del maestro c'era anche il genipodio. (Cercare la etimologia). Il genipodio consisteva in ciò, che durante tutta la quaresima lo scolaro, oltre al legno, portara al maestro qualche provvigione alimentare, ova fresche o sode, formagrgi e caciaiuole e via dicendo. Col pieno consenso delle famiglie, anzi non essendovi pur uno che si sogrnasse potersi far la cosa in altro modo, lo insegnamento andava avanti a furia di staffilate.

Da Condove, come da tutta la valle di Susa, i fanciulli destinati a proseguire negli studi, imparato il leggere e lo scrivere, ciò che avveniva piuttosto tardi, eran messi in collegio. 
Due collegi si contendevano il merito di educare quei fanciulli, il collegio di Giareno e quello di Susa. Il primo sopratutto era numeroso e accoglieva giovani da varie parti del Piemonte. La bellezza del luogo, la bontà del clima e il valore dello insegnamento concorrevano a dare a quel collegrio una grande e meritata riputazione. L'insegnamento consisteva tutto nel latino, salvo negli ultimi anni, che ora diciamo del Liceo e che allora si dicevano di Filosofia, che ora son tre e allora erano due, nei quali si insegnavano gli elementi della logica e dell'etica, della fisica e della greometria. Quest'ultima, sebbene più limitatamente che non oggi, s' insegnava a dovere; la fisica s'insegnava pochissimo, la logica e l'etica s'insegnavano in quel modo in cui si possono insegnare la logica e l'etica. Ma il latino, e anche le lettere italiane, compatibilmente colla età degli alunni, si insegnavano molto bene. La parte più larga era fatta al latino fin dalle prime scuole, e non credo d'essere fuori del vero nello asserire che per questo rispetto non si è progredito oggi in comparazione di quel tempo. Rimarrebbe ora da domandare quale e quanta fosse la bontà di quel metodo, ma non è questo il luogro di rispondere a una tale domanda.

Probabilmente Giovanni Francesco Re avrà fatto gli studi secondari nel collegio di Giaveno oppure in quello di Susa. Suppongo ciò, ma non posso asserire nulla, perchè le ricerche fatte non mi diedero nessun risultamento. Certo è che egli imprese 
poi a Torino e compi gli studii della medicina, mostrando subito una specialissima vocazione per le scienze naturali, vocazione la quale fini per prevalere in lui, per modo che egli la segui poi pienamente.

Fra le scienze naturali, siccome già sopra ho accennato, la sola che aresse un insegnamento e materiali di studio era la botanica, e il giovine studente si applicd con ardore allo studio di questa scienza. L' orto botanico del Valentino era bene accudito e ricchissimo di piante e il Re si applicò a conoscere a una a una quelle piante; inoltre fin dal primo anno degli studi incominciò a farsi un erbario, il quale sempre arricchì durante i cinqui anni del corso, per modo che appena laureato egli si trovava già possessore di un ricco materiale che tanto più gli riusciva prezioso, per ciò che se l'era fatto tutto da sè e ne aveva personalmente determinato ogni esemplare.

L'amore delle scienze naturali era cosi vivo in quel giovinotto che, mentre pure con molta lode dava opera allo studio di tutte le materie del corso, si rese anche famigliari le cognizioni fondamentali della mineralogia e della zoologia. Di quest'ultima scienza studiò segnatamente con predilezione due parti, la entomologia e la ornitologia, tutto ciò sempre praticamente e applicato alla sua valle nativa; fece anzi uno studio degli uccelli di val di Susa che fu ben accolto dall'Accademia delle Scienze di Torino. 
Appena laureato, siccome egli pensava a fare il medico, si occupò di studi clinici e patologici, e imprese l'esercizio della medicina nella città di Susa.

Una traccia di questo periodo della sua vita scientifica si ha in un suo volumetto stampato a Carmagnola nella stamperia di Pietro Barbier nell'anno 1797. Questo volumetto è intitolato: Riflessioni soria alcuni articoli della mura dottrina medica di Bronn. Tutto questo scritto si aggira sulla debolezza indiretta, sull'azione direttamente debilitante del freddo e sulla virtic sempre eccitante dell oppio. Per quanto consentivano tal sorta di argornenti e il modo di trattar le quistioni mediche allora in voga, questo libro fin apprezzato al suo tempo. Al. lora il dottor Re era rappresentante il protomedicato della città e provincia di Susa, posizione elevata e tanto più rla notare in così giovane età e in un tempo in cui la grioventù per gli uffici importanti era tutt'altro che una raccomandazione.

Venuto il governo francese si moltiplicarono le scuole per la istruzione secondaria e vi ebbe un posto la storia naturale. Il dottore Re si sentiva fatto per l'insegnamento e in particolare per l'insegnamento della storia naturale. Fbbe nel collegrio di Carignano il doppio insegnamento della matematica e della storia naturale.

La qualità di professore di matematica fece sì che egli pubblicasse poi una Istruzione facite e po. polare sul nuoro sistema delle misure e dei pesi. 
Questo volumetto aveva per scopo di far conoscere il sistema metrico ed è in gran parte condotto in forma di dialogo; fu tenuto in molto pregio per la limpidezza del dettato. Venne stampato a Carmagnola nel 1809 dalla stamperia di Pietro Barbier.

Ma lo insegnamento della storia naturale gli consentiva allora e anzi gli imponeva di volgersi tutto quanto ai suoi studi prediletti, e nella botanica si spinse avanti con tutte le sue forze, tanto nella parte teorica quanto nella pratica, e riusci veramente ad acquistare in essa cognizioni profonde.

Tutti gli anni, ogni qualvolta gli veniva concesso, da Carignano si recava a Condove e qui passava la maggior parte del suo tempo di vacanza, e quindi i mesi dell'autunno e i griorni del Natale e della Pasqua. A Condove non facera altro da mane a sera che erborizzare e studiare le piante raccolte. Si trovava perció, come ho detto fin dal principio, in una situazione felicissima. La valle di Susa, solcata dalla Dora per tutta la sua lunghezza, procede flessuosamente, ora stretta ed ora ampia, fra montagne con culmini elevati e passaggi di minor altezza, vertici frastagliati e pendii rocciosi, per una quarantina di chilometri nella direzione da ponente a levante, dal Moncenisio fino a Rivoli e Casellette dove è lo sbocco della valle e comincia la pianura di Torino percorsa per altri dieci chilometri circa dalla Dora, che va a sboccare nel Po dopo d'aver toccato la parte a Nord della città. 
Stupendi massi erratici di varia mole, qualcuno di mole imponentissima, giacciono sparsi allo sbocco della valle. Qui è la vegetazione della pianura, poi su pei pendii quella vegetazione diversa che colpisce tanto l'occhio del volgo, castagni e faggi, poi larici e pini, poi rododendri e poi licheni e muschi ed altre minutissime crittogame sotto e sopra la neve. Ma se il volgo giustamente si meraviglia di questo variare della vegetazione, guardato solo nelle sue forme più vistose, incomparabilmente maggiore è la meraviglia del botanico che cerca in quelle piante che non colpiscono l'occhio del volgo, nei fiorellini della vegetazione erbacea, le differenze a seconda delle altitudini e della varia esposizione dei pendii al sole e al vento.

Era un campo nuovo della scienza, era un mondo ignoto e attraente che si spiegrava agli occhi del giovane botanico che non riusciva a saziarsi dal contemplarlo. Perciò, ripeto, egli percorreva infaticato per ogni recesso la sua bella valle, da quei tratti intorno a Susa dove appare come un'oasi di vita meridionale, e cresce il mandorlo e neregrgia l'uva, fino alle cime eternamente nevose del Rocciamelone, ove si trovano colle temperature certe produzioni naturali che ricordano le plaghe gelate delle zone circumpolari.

Io mi fermo sopra ciò perchè veramente ne vale la spesa; certi luoghi presso Susa, per la espo. sizione pienissima al Sud e il riparo dai venti 
freddi, presentano prodotti vegetali e animali al tutto diversi da quelli dei luoghi vicini, e anche vicinissimi; si trovano là insetti e molluschi della Liguria, si trovano certe condizioni e certe produzioni naturali che ricordano le Isole Belle nel Lago Maggiore. In queste isole, per spiegare la eccezionalità del clima e dei prodotti naturali, si ebbe ricorso al calore centrale; si disse che il lagro è profondo, in certi luoghi profondissimo; che a quella maggior profondità il calore centrale deve già farsi sentire, che scaldandosi l'acqua nel profondo porta salendo quella più elevata temperatura alle spiagrge cui bagna. Questa spiegazione, la quale è tutt'altro che immune da obbiezioni per le Isole Belle, non fa per quelle singolari oasi di vita meridionale in val di Susa. Fatto sta che la cosa è cosi; non è d'uopo poi insistere sull' altro fatto che alle maggiori altitudini sui monti col clima si trova la vita delle terre settentrionali.

Il prof. Re, percorrendo diligentissimamente nel modo detto sopra il contorno e la valle di Susa e i vertici dei monti che la serrano, trovò una quantità di specie vegetali tanto nurnerosa che ne provò grande meraviglia. Lo Hudson, in Inghilterra, nel fare la Flor delle Isole Britanniche, aveva annoverato 1600 specie. Il prof. Re ne areva raccolto 1682 in val di Susa.

Allora egli pensò a pubblicare la Flora Segusiensis, quella appunto che qui ora si ristampa tradotta. Quella pubblicazione in latino venne fuori 
nel 1805, con una prefazione nella stessa lingua, che si dà pure in questa edizione tradotta, e che ha la dats da Condove del giorno 3 settembre di quell'anno. La stampa fu fatta in Torino dalla tipografia di Bernardino Barberis, in typographorum vico, $\mathrm{n}^{0} 272$.

Non è còmpito mio il dire dei meriti di questo lavoro; tutti i naturalisti sanno quanto grande sia il valore di una Flora locale, quando è fatta con accuratezza, con coscienza e con dottrina; il valore cresce col tempo, e ciò si dimostra qui appunto ora colla nuova edizione. Il plauso degli intelligenti fu grande compenso all'opera del giovane botanico e incitamento a studi ulteriori.

In questi studi egli prosegui alacremente senza dubbio; qui tuttavia, nella sua vita, m'incontro in una lacuna: non ho potuto sapere che cosa egli abbia fatto dal tempo in cui stampó la Flora Segu. siensis fino al 1818. i probabile che sia rimasto professore a Carignano per tutto il tempo in cui durò in Piemonte il governo imperiale di Francia, vale a dire fino al 1814. Ma colla ristaurazione sabauda fu tolto, insieme con tante altre cose, l'insegnamento della storia naturale nei licei. Allora il professore Re dovette certamente lasciare Carignano.

Il governo trancese aveva istituito in Piemonte, o piuttosto ricostituito, una scuola di veterinaria. Dico che il governo francese aveva ricostituito e non istituito, una scuola di veterinaria in Piemonte, percliè la istituzione di una scuola veterinaria in 
questa provincia italiana aveva avuto origine molto anteriormente e di poco aveva tenuto dietro alla istituzione in Francia della prima scuola di veterinaria. Come in Francia il nome di Claudio Bourgelat vivrà immortalmente, e non solo in Francia ma in tutto il mondo, per aver quell'uomo insigne proposto, promosso e compiuto la istituzione di una scuola veterinaria, la prima che fosse mai stata, cosi il Brug’none è benemerito in Italia, e appunto in Piemonte, dove la scuola veterinaria che fu istituita fu di gran lunga la prima in Italia e seconda solo a quella di Francia.

La prima scuola veterinaria in Italia fu in Piemonte, in un paesello poco discosto da Torino, dalla parte di ponente, luogo di caccia dei principi di Casa Savoia, e perciò chiamato Venaria Reale. La scuola della Venaria fu fondata nell'anno 1769. Quella scuola non diede gran frutto per questa principale ragione che gli allievi di essa non avevano avuto nessun ammaestramento preliminare, ed erano per lo più maniscalchi dei reggimenti, sovente al tutto illetterati. La rivoluzione fece sì che quella scuola si chiudesse, e il governo francese, siccome ho detto, la riaperse al Valentino, presso Torino (1) nell' anno 1800 ; due anni dopo

(1) La storia della scuola veterinaria del Piemonte fu scritta da parechi; citeró solo il lavoro del prof. cav. Domenico Vallada, attuale direttore di essa, lavoro meritevole per accuratezza di ricerche e di dati, e ricco di ragguagli, pubblicato in Torino nel 1872. " La Scuola veterinaria del Piemonte. Saggio storico della medesima, dall'epoca di sua fondazione (1769) a' tempi attuali." 
la aggregò alla Università; ma, a malgrado degli nomini insigni che vi davano opera allo insegnamento, quella scuola non fece che deperire, tanto che era a un dipresso cadavere nel 1814, quando venne la ristaurazione.

In breve la Casa di Savoia pensò a ricostituire in Piemonte la scuola di veterinaria e scelse per essa novellamente il luogo dove primieramente l'aveva collocata, la Venaria Reale. La nuova scuola di veterinaria alla Venaria Reale fu istituita nell'anno 1818.

Qui ritroviamo il professore Giovanni Francesco Re. Probabilmente, dopo cessato il dominio francese, egli si era ritirato a Condove, proseguendo i suoi studi botanici, e tenendosi in rapporto coi suoi amici dotti, e taluni anche potenti. Taluno di questi amici, probabilmente, lo propose al governo siccome insegnante mella scuola della $\mathrm{Ve}-$ naria, e certamente fu quella per ogni rispetto lodevolissima e benefica proposta. La nomina del Re fu a professore di materia medica, collo incarico della ispezione ed economia della farmacia; inoltre egli doveva istruire nel primo e secondo anno gli alunni intorno agli elementi della fisica, della chimica e della botanica; nel terzo anno insegnava la materia medica veterinaria e la botanica pratica; nel quarto ed ultimo continuava a svolgere il trattato di materia medica e dava inoltre precetti di terapeutica.

Di tutti questi insegnamenti ed uffici il profes- 
sore Re si disimpegnava coscienziosamente e degnamente; ma la parte di cui si occupava di più, sia come studioso, sia come insegnante, era, naturalmente, la botanica.

La Venaria è luogo molto acconcio per le collezioni botaniche; giace ai piedi di collinette selvose, che per una decina di chilometri occupano il tratto dove cominciano le montagne. Dalla Venaria al Musiné, salendo fino alla cima del monte, si può far la gita in una giornata, da un buon camminatore. Parlo per esperienza personale. Ora il Musiné è celebre nei fasti della botanica piemontese e menzionato in tutte le flore per la ricchezza mirabile della sua vegetazione. Il Musiné è allo sbocco della valle di Susa, tantochè il professore Giovanni Francesco Re potera ancora dalla Venaria sentir l'alito del vento della sua valle nativa. Dalla cima del Musiné, procedendo dal sud al nord, si elevano parecchie creste di non molto grande altezza, con pendii pienamente solegrgiati, e quindi essi pure rigogliosi di varia vegetazione. Così è di tutto quel tratto a sud che sta tra la valle di Susa e la valle di Lanzo, con piccole vallette intermedie. Son luoghi pieni di bellezza, e nello stesso tempo pel naturalista campo vasto di studi. I colli selvosi che dal piede del Monte Basso vengono fino alla Venaria, sono interrotti da vallette, torrenti, ruscelli e fontane, e s'intende che vi debba essere pure molto varia e ricca la regetazione. Ciò vuole esser detto principalmente 
clei boschi intorno alla Mandria, a poca distanza dalla Venaria. I piani poi immediatamente intorno alla Venaria, le rive della Ceronda e della Stura, offrono pure campo al botanico per un ricchissimo bottino. Fra la Ceronda e la Stura, lungo la strada che mena dalla Venaria a Caselle, ma molto più accosto alla Venaria, v'ha un punto cui i contadini del luogo chiamano i Tre buchet, dove muore la collinetta nella aperta pianura e dove si vengono a raccogliere con mirabile varietà i prodotti vegetali della montagna e della pianura, e che il Re prediligeva sopra ogni altro e visitava ogni griorno piu d'una volta, fino all' ultimo della sua vita. Curro, spossato, cadente, travagliato dalla tosse, io lo vedeva in tal modo ritornare dai Tre buchet poco prima che morisse. Egli mi prendeva allora per mano e mi spiegava quanto felice fosse quel sito per trovarvi i fiori più rari, e mi raccomandava di visitarlo poi assiduamente $\mathrm{i}$ giorni in cui avessi incominciato lo studio dei fiori. Io era allora un ragazzo, aveva appena dieci anni; ma pure, per quel fatto della persistenza tenace nella memoria dellc prime impressioni, io ho presente ora il suo aspetto, sento il suono della sua voce, ricordo l'amorevolezza che mi dimostrava. Ricordo il bene che si diceva di lui da quanti lo conoscevano, e il fatto che raccontavano i suoi vecchi amici dello avere egli, quando era giovinetto, camminato a piedi quaranta miglia per restituire sette soldi e mezzo che s'era fatto imprestare. 
Ricordo un'altra cosa, ed è che tutti gli allievi della scuola veterinaria avevano allora ciascuno il suo erbario, tutti conoscevano le piante del contorno e delle montagne vicine, tutti erano botanici. Io non credo che ci sia stata mai nessuna scuola dove si sia tanto studiata la botanica da tutti gli alunni, e tanto sia stata imparata. Molti di quegli alunni diventarono poi segnalati in altri rami della scienza, diedero opera all'inseg'namento, si fecero conoscere con pubblicazioni, di anatomia, di fisiologia, e via dicendo; ma tutti conservarono il gusto della botanica, e per tutta la rita continuarono ad accudire e aumentare il loro erbario compiacersi di esso. Chiunque sappia che cosa sono gli studî, chiunque abbia potuto riconoscere tutto il vantaggio che trae la mente per gli altri stuli da uno studio ben fatto, può comprendere quanto fosse in quel tempo benefica l'opera di questo professore.

Dalla Venaria il Re si spingeva nelle sue escursioni botaniche non solo fino a Torino, ma anche su per la collina che tanto pittorescamente sovrasta alla città, sollevandosi a notevoli altezze. Cosi imprese uno studio della vegretazione della collina comparativamente a quella della montagna e, come frutto di queste sue escursioni, pubblicò nel 18251826 una Flora Torinese non inferiore per merito alla Flora della valle di Susa. Pubblicò pure in quel tempo una Dissertazione sul Lycopus europers come succedaneo della china china. Molto piu arrebbe 
voluto pubblicare; ma egli voleva compiere coscienziosamente tutti i suoi uffici, e ciò gli prendeva molto tempo. Trovava poi nelle escursioni, segnatamente in montagna, sia per le impressioni diverse, sia anche per la stanchezza fisica in cui si induceva, un conforto a certi suoi dolori morali. Un suo figliuolo si era fatto frate ed egli aveva provato di ciò molta pena. Sovente, quando tenendomi per la mano mi menava ai Tre buchet, egli mi diceva:

- Bambino mio, ricordati di queste mie parole; qualunque cosa ti dicano, non t'indurre mai a farti frate. -

Io non capiva allora il significato di quella raccomandazione, che non mi doveva servire a gran cosa, perchè nessuno si prendeva la briga di consigliarmi il convento, ed io non ci pensava spontaneamente. Capii più tardi il significato doloroso di quelle parole. La moglie del professore Re era di famiglia nobile; apparteneva ai conti di San Secondo; molti anni dopo il nascimento del figliuolo frate, lo fece padre di una figliuoletta, cui diedero il nome di Sofia. Il nascimento di quella bambina aveva tenuto dietro di poco al mio, ed era stato convenuto fra i grenitori delle due parti che ci avrebbero sposati insieme. Da quella età di dieci anni non l'ho più veduta nè più ne ho inteso parlare.

Un grave dolore venne poi a colpire il professore Re e forse ebbe non poca parte nella sua 
morte, e fu la deliberazione in cui renne il Governo di trasportare la scuola veterinaria dalla Venaria a Fossano. Tutti i professori della scuola furono desolati di quella deliberazione; affranto e malaticcio, egli la sentì più dolorosamente di tutti. La scuola fu trasportata nel 1834, ma il Re non visse fino a quel giorno. Fgli mori il giorno 2 di novembre dell'anno 1833. Io ricordo il dolore che quella morte produsse nei colleghi suoi e nei suoi scolari, e posso ben dire che quel dolore fu grande.

Il dolore di quella morte fu sentito ben oltre la piccola cerchia del paesetto dove fu sepolto. Le accademie scientifiche più segnalate d'Italia e di altre nazioni lo avevano a socio, i cultori più insigni della scienza erano in corrispondenza con lui; tutti giustamente si dolsero dell' averlo perduto. Quel bene che si dice ogni griorno degli uomini morti di recente, parlando della loro intima vita, posso ben dire io ora di lui, morto da tanto tempo: fu veramente un uomo buono, un uomo dabbene, sommamente modesto, alieno da brighe, avverso agli intrighi, amante sovra ogni cosa del vero e del bene. Fu di statura piuttosto piccolo, di corporatura sottile, pallido d'aspetto, curvo negli ultimi anni della sua vita. Guando non parlava di scienza, parlava delle grandi cose che aveva veduto e cui avera anche partecipato, e il passagroio dell'esercito francese pel Cenisio, e l'opera sua di medico in quel tempo, erano rimembranze di cui grandemente si compiaceva, e di cui 
spesso mi teneva parola. Non so trattenermi dal ripetere ancora una volta che quel vecchio di sessant'anni, taciturno in generale e poco espansivo, si compiaceva singolarmente a parlare con me, fanciullo di dieci anni, e raccontarmi i fatti della occupazione francese.

Il professore Re aveva un erbario preziosissimo, sia per la quantità dei materiali, sia per la precisione delle indicazioni, sia per la copia dei tipi. Quell' erbario, morto il Re, fu acquistato dal professore Maurizio Reviglio, di Rivoli, che gli era scolaro carissimo, e gli succedette nell' insegnamento andando colla scuola veterinaria a Fossano. Il professore Maurizio Reviglio entrò poi nell' insegnamento universitario e fu per molti anni professore a Sassari e rettore di quell'Università. Nel lasciare Sassari, cio che segui recentemente, essendo ora venuto il Reviglio a prendere in Torino un po' di quel riposo di cui è tanto degno dopo una vita nobilmente spesa, fece dono delle sue collezioni botaniche, e quindi anche dell' erbario del Re, all'Università di Sassari. L'animo generoso del professore Reviglio, di cui egli dà quotidianamente non dubbie prove, si manifesto anche per quell' atto. Non so trattenermi tuttavia dal considerare che il vantaggio che può ritrarre Sassari da quell'erbario non è tanto grande quanto è quello che potrebbe ritrarne Torino. Le specie raccolte dal Re sono tutte di val di Susa e del contorno di Torino; molte, come ho detto, tipi delle sue de- 
scrizioni; tutte autentiche e sicurissime; pel botanico torinese quell' erbario sarebbe quindi un sussidio sommamente prezioso. L' erbario dell'orto botanico di Torino ha invece un gran numero di esemplari della flora sarda del Moris, che starebbero molto bene a Sassari. Forse di buon accordo fra l'Università di Sassari e l'Università di Torino si potrebbe fare un ricambio giovevole a entrambe. Con questo voto io chiudo il mio cenno intorno al professore Giovanni Francesco Re, e ringrazio l'avv. Felice Chiapusso, che, invitandomi a farlo, mi diede una prova di stima di cui gli sono molto grato, e, nello stesso tempo, fece in modo che io abbia dovuto riportarmi col pensiero ai lnoghi e agli anni della mia fanciullezza, che tanto più volontieri si ricordano quanto più si è avanti nella vita.

Mrchele Lessona. 



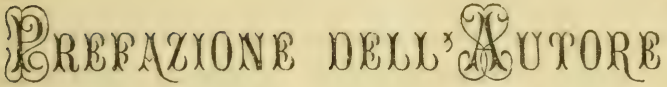

In nessun'altra scienza, tranne la medicina, io ho posto tanto amore, quanto ne posi nelle scienze naturali. Fin dai primi anni della mia grioventù ho impiegato, per quanto mi è stato possibile, tutto il tempo avanzatomi dallo studio ed esercizio dell'arte salutare e consentitomi dai doveri di Professore di Filosofia, a ricercare ed esaminare ogni corpo appartenente ai tre Regni della Natura.

Trovandomi a Torino addetto agli studi di Medicina, non mancava di recarmi ogni giomo all' Orto Botaniço ed ivi porre ogni mia cura nell'acquistar conoscenza di tutte le piante, di cui summamente abbondava esso in quell'epoca, cosicchè giunsi nello spazio di cinque anni a farmi una ricchissima raccolta di erbe.

Ritornato nella provincia, mia terra nativa, e chiamato dal Municipio di Susa ad esercitare la professione di Medico, non solo non trascurai gli studi botanici, ma presi altresì a coltivare con amore l'Ornitologia, l'Entomologia, ed un poco anche la Mineralogia.

Per lo che nella primavera e nell' estate io faceva non pochi viaggi per la provincia fin sulle più alte 
giogaie delle vicine Alpi e notava in un libro ogni cosa che giornalmente andava osservando e scoprendo; ne fanno fede gli Atti delle Scienze, segnatamente le Miscellanee Botaniche del Chiar. ${ }^{m o}$ Prof. Balbis, in cui trovai parecchie piante non ancora vedute da altri e da me raccolte in quel di Susa.

Molte ragioni secondavano questa mia inclinazione allo studio delle Scienze naturali, ma erami sommamente di sprone il vedere come il suolo di Susa più d'ogni altro fosse riceo di cose riguardanti quelle scienze; come pure mi animava la costante amicizia e corrispondenza epistolare, sì del lodato chiar. ${ }^{\circ}$ Prof. Balbis, che dell'Egregio Ignazio Molineri, custode dell'Orto botanico.

Con quest'espertissimo nomo visitai nell'anno 1803 il Moncenisio e le vicine Alpi, e colla guida di lui mi venne fatto di raccogliere moltissime specie in quel tempo rarissime e non scoperte da me fin allora.

La copia delle cose naturali raccolte in due lustri crescendo ogni giorno di più, io pensava di dar alla luce la Flora e la Fauna di Susa; ma accortomi di quante fatiche ancora abbisognasse perchè fosse in grado di soddisfar pienamente ai desiderii dei cultori della Storia Naturale, mi posi invece a siffatti studi con maggior lena ed alacrità. $\mathrm{E}$ quando finalmente $\mathrm{mi}$ era deciso di por mano all'opera, venni altrove chiamato dal chiar. ${ }^{\circ}$ Laville, allora Prefetto della Provincia del Po, e mi fu. affidato l'incarico d' insegnar Matematica e Storia Naturale nel Collegio di Carignano, lungi dalla patria mia.

Desideroso oltre ogni dire di far cosa utile alla patria, io stava in dubbio se dovessi o no rendere di pubblica ragione ciò che aveva ottenuto da diuturni ed improbi lavori, ma altrimenti pensarono i miei amici, tra i quali sono da annoverarsi l'illustre Prof. di Anatomia Giulio, 
ora Profetto del Dipartimento della Śesia e l'egregio Prof. di botanica, Balbis.

Mosso dalle esortazioni di questi Sommi, terminato l'anno, composi un libro sull'Ornitologia di Susa e l'offrii all'Accademia Imperiale delle Scienze di Torino. E fu a quell'insigne Consesso cotanto accetto e grato lo specchio delle mie fatiche intorno alle cose naturali, che quei Dottissimi decretarono unanimamente di farlo stampare ed inserire negli Atti dell'Accademia.

Spinto da questo nuovo incitamento io più non esitai a dare alla luce la Flora di Susa; la stampai in foggia di catalogo, affinchè il libro riescisse di minor mole possibile, indicandovi i luoghi natii delle piante, e vi agogiunsi puranco i termini proprii della medicina, affinchè tornasse di giovamento ai Medici ed ai Farmacisti. Nè ho taciuto, credendolo necessario, qual parte della pianta si dovesse adoperare, notando eziandio con un asterisco quelle piante che in questi tempi sono disusate (1).

Io ti presento, o Lettore, in piccola mole condensata cuna grand'opera, giacchè ove taluno desiderasse più ampie descrizioni delle specie potrà facilmente riscontrar quegli Autori, dai quali, come apparisce nel mio libro, furono loro imposti i nomi. In esso si contengono non solo $i$ frutti delle assidue fatiche durate da me per lunghi anni, ma altresì gli ammaestramenti di altri chiarissimi personaggi Allioni, Bellardi e Balbis, i quali scorsero ed esplorarono tutto il territorio di Susa, e spe-

(1) Nella traduzione abbiamo, invece dell'asterisco, scritto Farmaco o Rimedio disusato in caratteri grandi del testo: abbiamo scritto le medesime parole oppure Disusato in farwracia a carattere piccolo delle annotazioni quando l'osservazione è nostra. 
cialmente del Botanico Ignazio Molineri. Del consiglio di questi Sommi io mi sono giovato nel mandare a compimento quest'opera, dacchè Essi, con cortesia senza pari, mi diedero contezza di molte piante, coll'aggiunta delle quali si è non poco accresciuta e felicemente condotta. a termine.

Chi non sa tra quali angusti confini è ristretta la Provincia di Susa? Eppure in più luoghi è sì grande la differenza e la varietà del clima, che facilmente il Botanico vi può trovare quelle specie che attecchiscono nei luoghi freddi ed umidi del pari con quelle proprie di clima caldissimi, di cui sarebbe vana la ricerca in altre terre del Piemonte. A questa varietà di clima della Provincia di Susa devesi attribuire la ragione, per la quale questa mia Flora faccia menzione di 1682 specie di piante, mentre nella Flora Inglese di Hudson non ne sono nominate che 1600 .

Condove, 3 Settembre 1805.

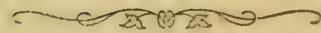




\section{A VVERTENZA}

Il testo è stampato in caratteri grandi, e nello stesso carattere, preceduti da un asterisco, i generi e le specie che furono aggiunte alla Flora del Re.

Tutto il resto, variazione di nomi, epoca della fioritura, varietà precedute da asterisco, comenti, ecc., stampato in carattere più piccolo, è del traduttore. Per. significa perenne. Ann. vuol dire annua. 



\section{Classe I. - DICOTILEDONI}

DIVISIONE PRIMA

Talamillore - (Talamifores)

Ordine 1. - Ranunculacee. (Ranunculacea Juss.)

Sez. - Trutif. per Acheni snonøsperni inateiscenti A) - Foglie opposte.

Gen. $1^{\circ}$ - Clematis Lin.

(Fiori bianchi)

Cl. recta Lin. - Lungo la Dora. Acre, caustica. Per. Giugno, Luglio.

Vitalbino it. - Clematite droite fr.

Caselette: Musiné.

Malamente nel testo Clematis erecta Lin. - Cl. erecta è di Jacq.-All. non di Lin.

Cl. Vitalba Lis. - Clematis o Viorne Off. - Ovunque nelle siepi. Acre, caustica.

Per. Giugno, Luglio.

Vitalba it. - Viorne: vigne blanche fr.

Le foglie contuse della Vitalba, come della specie precedente, applicate alla pelle riescono epispastiche. Però questa 
proprietà irritante si manifesta a stagione avanzata. I germogli della Vitalba sono mangiati impunemente dalle bestie $e$ in taluni paesi anche dagli uomini.

I semi della Vitalba sono vantati da Krauss come potentemente diuretici.

\section{Gen. $2^{\circ}$ - Atrageme Lin.}

Atragene alpina Lis. Vabies vern. - Ovunque nei monti di Bussolino, nel Cenisio e verso l' Assietta.

Per. Giugno, Luglio.

Vitalbino dei sassi it. - Atragène fr.

Musiné (Re Fl. Tor.). Dal colletto di Meana al colle delle Finestre.

Bellissima pianta, che decora le rupi delle nostre Alpi. Ha sepali grandi violetti.

Molti botanici moderni considerano, e forse a ragione, come stami trasformati (staminodi) i cosi detti petali dell'Atragene.

B) - Foglie tutte radicali: tre brattee verticillate sul caule (involucro) più o meno lontane dal fiore: prossime, caliciformi, nell' A. hepatica.

Gen. $3^{\circ}$ - memone Lin.

1. Acheni scodati.

* Fiori violetti, raramente rosei o bianchi.

\section{Anemone hepatica Lrs. - Hepatica nobilis - Hepatica, Trinitas, Herba trinitatis Off. - Comune nelle selve.}

Per. Aprile Maggio.

Erba trinità it. - Anémone hépatique fr.

Un giorno fu ritenuta come rimedio alle malattie epatiche; oggi è farmaco disusato. 
* Fiori bianchi almeno superiormente.

A Baldensis Lin. Mant. (A. Fragifera Re-TVulf). Nei pascoli sassosi del Cenisio.

Per. Luglio, Agosto.

Anemone fragolino it. - A. du Mont Baldo fr.

Cenisio a Ronche (Negri).

Presso le antiche fortificazioni del Colle delle Finestre.

Nel testo A. Frag. Lin. dal Murr. Syst.

A. nemorosa Lin. - Ovunque nelle selve.

Per. Marzo, Aprile.

Auemone bianco it. - A. sylvie fr.

A. narcissiflora LrN. - Abbonda nei monti di Giaveno, nei prati del Cenisio e presso li Gonté nei monti di Bussolino.

Per. Giugno, Agosto.

Anemone narcissino it. - A. à fleurs de narcisse fr.

$\mathrm{Ha}$ i fiori disposti ad ombrella; pianta alpina bellissima.

Rocca o passo del Mouton nei monti di Giaveno (De Fllippi).

A. sylvestris Liv. - Trovata da Ignazio Molineri nei monti sopra Coazze.

Per. Maggio, Giugno.

Specie assai dubbia come pertinente alla Flora. Per quanto sappiamo, A. sylvestris non è pianta italiana. - Allioni scrive che una volta sola (semel) occorse nella località accennata. (Fl. Ped.).

*. Fiori giallo-dorati.

A. ranunculoides Lin. - Frequente in primavera presso Susa e specialmente nei prati delle Blaccie e di Croale.

Per. Maggio.

Anemone dei boschi it. - A. à fleurs de Renoncule fr. 


\section{Acheni codato-piumosi.}

* Fiori bianchi, almeno superiormente.

A. vernalis Liv. - Abbonda nei prati alpini di Monpantero e del Cenisio, come nei pascoli della Comba sopra Chianoc.

Per. Maggio, Giugno.

Anemone primaticcio it. - A. du printemps fr.

A. alpina Lin. - Copiosa nei prati pressu la fabbrica del marmo; nel Cenisio e nelle Alpi di Giaveno.

Per. Giugno, Luglio.

Anemone d'Alpe - A. des Alpes fr.

* * Fiori violetti o purpureo-violetti.

A. pulsatilla Lin. - Pulsatilla Off. - Niente di più volgare nei pascoli sterili intorno a Susa.

Per. Aprile, Maggio.

Anemone pulsatilla it. - Coquelourde fr.

Anemone Halleri ALL. - Volgarissima nel Cenisio sopra la Posta: sopra la Gran Croce: nella contrada di Fossimagna, e presso Susa ad oriente della roccia detta Tre pene.

Per. Maggio, Giugno.

An. frastagliata it. - A. d'Haller fr.

In generale le Anemoni sono acri ed irritanti, atteso una sostanza speciale di esse caratteristica (Anemonina) che contengono, specialmente allo stato fresco. Una virtù terapeutica (malattie della pelle e della vista) pressochè prodigiosa fu attribuita all' A. Pulsatilla, massime dai medici omeopatici. - Per essi la Pulsatilla era quasi una panacea; il suo grido oggi è di molto scemato.

Le Anemoni sono rifiutate istintivamente dal bestiame. 
C) - F'oglie o tutte radicali o radicali e cauline, alterne. GeN. $4^{0}$ - Thaliet arem Lin.

* Acheni triangolari alati, lisci, pedicillati.

Th. Aquilegifolium Lin. - Comune nelle selve subalpine.

Per. Maggio, Giugno.

Talittro colombino it. - Pigamon à feuilles d'Ancolie fr. *' Acheni non alati, oblunghi, striati, sessili.

Th. foetidum Lis. - Cenisio.

Per. Luglio, Agosto.

Pigamo fetido it. - Pigamon fétide fr.

Alle Blaccie ed a Gravere (Negri).

Th. minus Liv. - Nei prati presso Susa, e nel Cenisio.

Per. Maggio, Giugno.

Erba Scopaia,it. - Petit Pigamon fr.

Th. flavum Lin. - Th. Off. - Nei prati sabbiosi ed umidetti. Eccoprotico.

Per. Maggio, Giugno.

Pigamo it. - Pigamon Jaunâtre fr.

Rimedio disusato.

* Th. odoratum Gren. e God. - Cenisio alla Ramasse (Bouvier).

Per. Luglio, Agosto,

Pigamo odoroso it. - Pigamon odorant fr.

\section{Gen. $5^{\circ}$ - domis LIN.}

Ad. Aestivalis Lin. (Ad. miniata Re-Ald.). - Abbonda nelle messi e specialmente di Foresto.

Ann. Maggio, Giugno.

Camomilla rossa it. - Adonide d'été fr. 


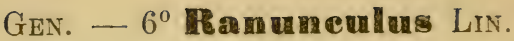

A) - Acquatici: petali bianchi con l'unghia gialla.

Ran. aquatilis Lin. - Presso le acque a lento corso o stagnanti. Acre come tutto il genere dei ranuncoli.

Per. Giugno, Agosto.

Ranuncolo soldinello it. - Renoncule aquatique fr.

Ran. pantothrix DC. (R. peucedanifolius RE-ALL.) - Nei fossi e nelle acque limpide.

Per. Aprile, Luglio.

Finocchio d'acqua it. - R. à feuilles capillaires fr.

$$
\text { B) - Terrestri, al più di siti umidi. }
$$

1. Petali gialli: Foglie semplici intere o quasi.

Ran. flammula Lin. - Nei prati umidi. Acre, velenoso.

Per. Giugno, Agosto.

Ranuncolo delle passere it. - R. Flammette fir.

Ran. ficaria Lin. - Chelidonia minor, Scrophularia minor, Ficaria Off. - Frequente nei prati irrigui. Abbonda presso Susa nei prati della Bretagna sotto l'Arnaudera. La radice fresca è diuretica.

Per. Marzo, Maggio.

Flavagello it. - Petite Éclaire fr.

Disusato in farmacia. Questa specie di Ranuncolo è forse la meno acre di tutte le altre: in qualche paese sono mangiate impunemente le sue foglie condite di olio e di aceto. 
Ran. gramineus LiN. - Raccolsi questa rara specie di Ranuncolo, e in copia, nelle rupi apriche delle Blaccie e la comunicai al chiaris. mio amico prof. di botanica Balbis, come si rileva dalla Miscellanea botanica dello stesso autore a p. 26. Trovasi pure al Monte Oliveto presso Meana. Radice e fiore acri.

Per. Maggio, Giugno.

Ranuncolo barbuto it. - R. à feuilles de gramen fr.

Foglie lanceolate intere a nervi paralelli : petali triangolari. A Gravere (Negri).

Ran. lingua Lin. - Sponde dei laghi d' Avigliana. Acre, velenoso.

Per. Giugno, Agosto.

Ranuncolo delle sanne it. $-R$. grande douve fr.

2. Petali gialli: Foglie più o meno partite, o composte.

\section{* Peduncolo solcato.}

Ran. bulbosus Lin. - Ran. tuberosus o bulbosus Off. - Ovunque nei prati e nei pascoli. Acre, caustico.

Per. Maggio, Giugno.

Lappio it. - R. bulbeuse, Rave de Saint-Antoine, Pied de coq fr. - (Sepali riflessi).

Ran. Sardous Crantz. (Ran. agrarius Re-Ald.) - Nei campi.

Ann. Maggio, Giugno.

Stroscione it. $-R$. des mares fr.

(Ran. philonotis Ehrh). - (Sep..riflessi). 
Ran. repens Lrs. - Nei prati umidetti. Acre, caustico.

Per. Aprile, Giugno. (Sepali patenti).

Stellettine it. - R. rampante fr.

* * Peduncolo cilindrico.

(Sepali patenti in tutte le specie).

Ran. auricomus Lin. - Il chiaris. Allioni scrisse che questa specie sia stata trovata nella valle di Oulx.

Per. Aprile, Maggio.

Bottoncini d'oro it. - R. à tête d'or fr.

Specie dubbia come pertinente alla flora di Susa. Re nota solamente che Allioni ci abbia tramandato ch' essa vegeta nella valle d' Oulx. Il testo dell' Allioni (Fl. ped.) non rischiara il dubbio. Allioni serive :

" La posseggo raccolta in Piemonte. Bartolomeo Caccia "la annovera fra le specie della valle d'Oulx ».

Ran. montanus Willd. (Ran. nivalis RE-JACQ.) In tutti i prati alpini.

Per. Giugno, Luglio.

Ranuncolo montano it. - $R$. des montagnes fr.

* B. Villarsii (R. Villarsii DC.) - Cenisio, pendii erbosi e sassosi sotto l'Eau blanche (Verlot).

Ran. lanuginosus Lis. - Luoghi montani presso le selve.

Per. Maggio, Giugno.

Ran. lanato it. - R. laineuse fr.

Ran. acris LrN. - In tutti i prati. Acre, caustico.

Per. Maggio, Giugno.

Batrachio it. - Ren. acre fr. 
Ran. arvensis Lis. - Frequente nei campi. Acre, caustico.

Per. Maggio, Giugno.

Ranunculo dei campi it. - R. des champs fr.

\section{Petali bianchi.}

" Foglie intere.

Ranunculus pyrenaeus Lin. Mant. - In tutte le Alpi.

Per. Giugno, Luglio.

Ran. lanciolo it. - R. des Pyrenées fr.

* Foglie partite o composte.

a) Fosselta dell' unghia dei petali squamata.

Ran. aconitifolius LIN. - Presso ai rivi e nei luoghi pingui del Cenisio, nei prati del Collet e nelle Alpi di Giaveno.

Per. Giugno.

Piè di cornacchia it. $-R$. à feuilles d'Aconit fr.

Ran. glacialis Lis. - Sommità del Cenisio presso le nevi in liquefazione. Velenoso.

Per. Luglio, Agosto.

Carolina it. - R. des glaciers fi.

M. Chaberton presso la cima.

b) Fossetta nuda.

Ran. alpestris Lis. - Raccolto intorno al Lago delle Alpi di Giaveno da Ignazio Molineri: comune anche nelle Alpi di Matthie, nel monte Clot de Brun presso il Vallon e nel Cenisio.

Per. Giugno, Luglio.

R. Alpestre it. - R. Alpestre fr. 
R. rutaefolius Lin. - Prati del Cenisio presso la Ramasse.

Per. Luglio, Agosto.

Ran. Coriandolino it. - R. à feuilles de rue fr.

Bella e rara specie alpina. Ha i petali a base aranciata. Cima d'Eau Blanche (Negri).

Sez. $\mathbb{P}^{2}$ - Fruttif. per eapsule polisperme deiscenti Gen. $7^{\circ}$ - Caltha Lin.

Cal. Palustris Lin. - Abbonda nelle Alpi presso le fonti. Acre, velenosa.

Per. Maggio, Agosto.

Calta it. - Populage. Souci des marais fr.

Sagra di S. Michele.

Dannosa al bestiame, se la mangia nei fieni. La pianta viva è rifiutata.

La Calta è l'unica specie di questa Sezione priva di petali. Ha 5 sepali petaloidi giallo-dorati.

\section{GEN. $8^{\circ}$ - Trollius LIN.}

Tr. europeus Lis. - Comune nei prati umidi alpini.

Per. Maggio, Agosto.

Luparia it. - Trolle fr.

Sagra di S. Michele nei prati. (De Filippi).

Ha petali da taluni tenuti per staminodi, piccoli, piani, e sepali petaloidi citrini.

Gen $9^{\circ}$ - Helleborus Lin.

Hel. foetidus Lin. - Helleborus foetidus o Helleboraster Off. - In tutta la Valle d'Exilles. Emetico, catartico, sospetto.

Per. Aprile, Maggio.

Cavolo di Lupo it. - Pattes de griffon; Pattes d'ours fr. 
Ha odore viroso ributtante: si adoperava un giorno come purgante. Una specie di Hel. (Hel. orientalis), che abbonda nell'isola di Anticira, era tenuta dagli antichi greci come rimedio della follia.

E rifiutato dal bestiame e, se da esso ingerito con altre erbe, gli riesce dannosissimo.

Hel. foetidus ha i petali (staminodi?) piccoli, tubolosi, e sepali verdi orlati di rosso cupo.

* H. Viridis Lrn. Da Almese a Rubiana (De Filippi).

Per. Fine di Marzo, Aprile.

Elleboro verde it. - Hellébore vert. fr.

Sepali totalmente verdi.

\section{Gen. 10 - Aquileging LIN.}

* Cornetti lunghi quanto i sepali con la punta a forma di amo.

A. vulgaris Lin. - Aquilegia o Aquilina Off. - Ovunque nei siti ombrosi dei monti o presso le acque. Velenosa.

Per. Maggio, Luglio.

Aquilegia it. - Ancolie: gants de Nôtre-Dame fr.

Farmaco disusato.

* B. Atrata (Aquilegia atrata Koch) Sagra di S. Michele nei prati. (De Filippi). - Nella specie i fiori sono cerulei, rosei o rar. bianchi; nella varietà violetto-scuri; in questa le foglie sono più profondamente partite ed i fiori più piccoli che nella specie.

* Cornetti piu corti dei sepali dritti, o areuati a punta non uncinata.

Aq. alpina Lrs. - Nei pascoli e nei prati macilenti del Cenisio. Rimedio disusato.

Per. Luglio, Agosto.

Aquilegia maggiore it. - A. des alpes fr. 
GEN. 11. - Delphiminum LiN.

D. consolida Lin. - Flores consolidae regalis Off. Fra le stoppie nei campi. Rimedio disusato.

Ann. Giugno, Agosto.

Cappucci it. - Pied d'Alouette fr.

Gen. 12. - Acomidum Lin.

* Fiori gialli.

Ac. anthora LIN. - Anthora o Antithora Off. - Visto nei prati alpini soleggiati sopra Ferrières dall' esimio Ign. Molineri. Acre, amaro, catartico, velenoso.

Per. Agosto.

Antora it. - Aconit Anthora fr.

Piccolo Cenisio (Bonjean in Bertol.).

Ac. Lycoctonum Lin. e Off. - Non raro nei luoghi alpini e montani. La radice è buona ad ammazzare i pidocchi.

Per. Luglio, Agosto.

Lupaja it. - Tue-loup fr.

* * Fiori cerulei o violacei.

Ac. cammarum LrN. - Nei siti ombrosi e freddi del Cenisio e alla Fabbrica del Marmo. Velenoso.

Per. Giugno, Agosto.

Cammaro it. - A. de Jacquin fr.

Elmo ampio elevato-conico; lato superiore dei follicoli pubescente. Fiore ceruleo.

* Ac. napelius Lin. Cenisio (?) (Balbis e Bonjean in Bertol.).

Per. Giugno. Agosto.

Aconito napello it. - Ac. napel, Casque de Jupiter, Capuche de moine fr. 
Elmo emisferico poco elevato; follicoli pubescenti o glabri. Fiore violaceo o bianco-ceruleo, rar. bianco.

* Aconitum cernuum Wulf. - Cenisio (?) (Balbis e Bonjean in Bertol.).

Per. Giugno, Luglio.

Aconito pannocchiuto it. - Ac. penché fr.

Nell'ordine delle Ranunculacee gli Aconiti sono i più venefici; dalle loro radici e specialmente del Napello si ricava un terribile alcaloide (l'Aconitina), della quale bastano pochi milligrammi ad estinguere un uomo. Dell' estratto semplice dell'Aconito napello, come dell' Aconitina, si vale la terapia moderna per combattere talune nevralgie, il reumatismo ed i dolori gottosi, sebbene taluni clinici, tra i quali il Cantani, siano avversi al suo impiego.

\section{Gen. 13. - Paeonia LiN.}

Paeonia officinalis Lin. - Paeonia Off. - Rupi della Sagra di San Michele.

Per. Maggio, Giugno.

Peonia it. - Pivoine fr.

Musiné, lato che guarda Susa (Re, Fl. Tor. sotto Paeonia peregrina).

La Peonia è velenosa. Una volta era molto in voga come rimedio antispasmodico. Oggi è pressochè disusata.

Sez. $\mathfrak{B}^{2}-$ Fruttif. per capsule baceiforma Gen. 14. - etaen Lin.

Ac. spicata LrN. - Aconitum bacciferum o Chrystophoriana Off. - Luoghi ombrosi dei monti. Velenosa.

Per. Maggio, Luglio. Attea it. - Actée fr.

(Fiore bianco -4 petali). 
Le radici di Attea partecipano delle-qualità acri degli Aconiti e furono un giorno adoperate a combattere le stesse malattie, in cui oggi si fa uso del Napello.

Ordine II. - Berberidee (Berberideae Vent.)

\section{Perberis LIN.}

B. vulgaris Lin. - Berberis Off. - Nelle siepi, nei pascoli; in copia presso les Ferrières. Le bacche acide sono rinfrescanti.

Arbusto. Maggio, Giugno.

Berberi it. - Épine vinette fr.

Le radici del Berberi danno una sostanza amarissima (il berberino) la quale venne adoperata con successo dal Maggiorani per ridurre i tumori splenici malarici.

Le bacche del Berberi contengono acido citrico e malico e sono grandemente adoperate in Francia sotto forma di sciroppi e conserve come toniche e rinfrescanti.

Ordine III. - Ninfeacee (Nympluaucece Salisb.) Gex. $1^{\circ}$ - Nymplnaea LiN.

\section{N. alba Lin. - Nym. alba o Nenuphar album 0ff.}

- Nei laghi di Avigliana. Fiori ipnotici.

Per. Giugno.

Ninfea it: - Nénuphar fr.

Lago di Caselette (Re, Fl. Tor.).

Fiori grandi bianchi, odoranti; splendida pianta degna di decorare le vasche dei giardini. Una volta i fiori e le radici furono adoperati come calmanti e anafrodisiaci. 
Gen. $2^{\circ}$ - Wrphar Suith.

* Nuph. luteum Sмiтtн. - Laghi di Avigliana. (De-Filippi).

Per. Maggio, Giugno.

Ninfea gialla it. - Nuphar fr.

Lago di Caselette (Re, Fl. Tor. sotto Nymphaea lutea).

Fiore giallo odorante, foglie grandi coriacee, bellissime.

Ordine IV. - Papaveracee (Papareracece DC.)

Gen. $1^{0}$ - Papaver Lin.

- Capsula ispida.

P. argemone LrN. - Nei campi.

Ann. Maggio, Giugno.

Argemone it. - Pavot Argémone fr.

(Capsula oblunga claviforme - Petali rosei).

P. hybridum Lis. - Intorno la Brunetta per asserzione del $\mathrm{Ch}^{\circ}{ }^{\circ}$ Allioni.

Ann. Maggio, Giugno.

Papavero spinoso it. - P. hybride fr.

(Capsula globosa - Petali purpurei).

* Capsula glabra.

P. Rhoeas Lin. - Papaver erraticum, rubrum Rhoeas Off. - Papaver vern. - Comunissimo nelle messi. Fiori diaforetici, subnarcotici.

Ann. Maggio, Luglio.

Papavero it. - Coquelicot fr.

Ł̀ molto dubbio (Cantani) che i fiori del papavero contengano morfina e narcotina. Comunque sia essi sono oggi in disuso ed hanno ceduto il posto al Papavero sonnifero originario dell' Asia e dell' Africa. 
P. dubium Lis. - Nei pascoli declivi.

Per. Maggio, Giugno.

Rosolaceio a mazza it. - P. douteux fr.

* P. somniferum LiN. - Coltivato in qualche giardino.

Ann. Giugno, Luglio.

Papavero bianco it. - Pavot fr.

Dalle capsule verdi incise di questa specie cola un liquido che poi si rapprende e si dissecca all' aria. È l'oppio usato in farmacia come calmante e antispasmodico.

Dall' oppio si estraggono varii alcaloidi, fra i quali primeggiano per virtù terapeutiche la morfina e la codeina. La morfina è veleno potentissimo.

\section{Gen. $2^{\circ}$ - Clnelidoming Tournf.}

Chel. majus LIN. - Chelidonium majus - Chelidonia major o Hirundinaria Off. - Freq. intorno alle case e sulle mura. Rad. diuretica.

Per. Maggio, Agosto.

Chelidonia it. - Chelidoine, Éclaire fr.

Farmaco non del tutto abbandonato dalla terapia moderna: radice e caule agirebbero come drastico potente. Il suo succo giallo atrofizza le verruche.

Ordine V. - Fumariacee (Fumariacea DC.) Gen. $1^{\circ}$ - Corydalis DC.

* Pedicilli molto piú brevi delle capsule.

C. tuberosa DC. (Fumaria bulbosa RE-LIN.) - F. bulbosa o Aristolochia fabacea Off. - Nelle siepi. Rad. amara.

Per. Marzo, Maggio.

Radice Cava it. - Corydalis Creuse fr.

Farmaco disusato. 
* Pedicilli lunghi quanto la capsula.

G. bulbosa DC. (Fumaria Halleri RE-WILLd). Raccolta da me copiosamente sul Cenisio allorquando percorsi quel monte in compagnia dell'esimio botanico Ignaz. Molineri.

Per. Aprile, Maggio.

Fumaria ditaruola it. - Corydale pleine fi.

\section{GEN. $2^{\circ}$ - vamaria LiN.}

F. officinalis Lrs. - Fum. Off. e vern. - Volgarissima nelle vigne. Amara, stomatica.

Ann. Maggio, Agosto. (Fiori rosei).

Fumaria fumosterno it, - Fumeterre fr.

Rimedio ancora in uso come amaro digestivo.

F. parviflora Lamk (Fum. spicata Re-All.) - Anche nelle vigne, ma più rara della precedente.

Ann. Giugno, Agosto. (Fiori bianchi).

Fumosterno sottile it. - Fumeterre à petites fleurs fr.

Nel testo F. Spicata Lin. dal sinonimo di Allioni (Ped.) o dal Murr. (Syst.) - F. spicata Lin. (Platycapnos spicatus Bernh. Parlat.) è tutt'altra pianta, e di altri siti. Re emendò l'errore nella sua Fl. Torinese, vol. $2^{\circ}$, pag. 3 , ove a F. Parviflora DC. Prodr., ch'è la nostra specie, dà per sinonimo F. Spicata All. Balbis. 


\section{Ordine VI. - Crocifere (Cruciferce Juss.)}

\section{Sez. 1 - Siliquose: Siliqua lineare deiscente} assai pin lunga che larga.

A) - Cotiledoni accombenti. (Cot. accumbentes) Gen. $1^{0}$ - Mathiolla R. Brown.

Mat. tristis R. Brown (Cheiranthus tristis RE-LiN.) - Raccolta dal Ch. Allioni nei siti aprici della Brunetta.

Per. Maggio, Giugno.

Violaciocca notturna it. - Matthiole triste fr.

Gen. $2^{\circ}$ - Nasturfiun R. Brown.

(Semi inegualmente biseriati).

* Fiori bianchi.

Nast. Officinale R. Brown (Sisymbrium Nast. Re-Lin.) - Nast. aquaticum Off. - Cresson vern. - Ovunque nelle acque a lento corso. Antiscorbutico.

Per. Giugno, Settembre.

Crescione it. - Cresson fr.

Questa pianta ha un succo piccante ed alquanto amaro. È gustoso alimento, e sino ad un certo punto un buon rimedio digestivo e antiscorbutico.

* Fiori gialli.

Nast. Pyrenaicum R. Brown (Sisymbrium Pyr. RELin.) - Prati e pascoli macilenti. In copia presso Giaveno.

Per. Giugno, Luglio.

Radicina sottile it. - Cresson des Pyrenées fr. 
Nast. sylvestre R. Brown (Sisymb. sylvestre RE-LiN.) - Eruca palustris Off. - Nei pascoli umidi. Antiscorbutica.

Per. Giugno, Luglio.

Radicina serpeggiante it. - Cresson sauvage fr. Farmaco disusato.

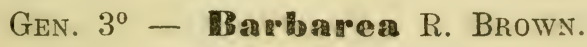

(Semi uniseriati)

B. vulgaris R. Brown (Erysimum Barbarea RE-LiN.) - Ovunque nei margini dei fossi e nei prati uriidi. Antiscorbutica.

Bien. - Per. Aprile. (Fiori gialli).

Erba di Santa Barbara it. - Barbarée, Herbe de Sainte Barbe fr.

Rimedio disusato.

\section{Gen. 4. - Teuroitis LIN.}

(Semi biseriati)

T. glabra Lis. - Intorno alle selve.

Bienn. Maggio, Giugno. (Fiore bianco-gialliccio.)

Baccellina verdemare it. - Tourette glabre fr.

\section{GEN. 5 - Arabig LiN.}

(Semi uniseriati).

(Fiori bianchi in tutte le specie, salvo nell' A. caerulea).

1. Semi sprovvisti di ala

o cinti di un' ala angusta dilatata solamente all' apice.

* Foglie cauline semplici, amplessicaule - cuoriformi.

a) - Foglie intere glabre.

A. brassicaeformis Wallroth. (Brassica alpina RE- 
Liv.) - Nella convalle presso il torrente sopra Foresto.

Per. Maggio, Giugno.

Brassica d'alpe it. - Arabette à feuilles de chou fr.

Moncenisio (Bouvier).

b) - Foglie piit o meno irsute e piu o meno dentute.

A. alpina Lin. - Nei prati alpini, specialmente nei pascoli ombrosi di Monpantero e del Cenisio.

Per. Maggio, Settembre.

Pelosella d'Alpe it. - Arab. des Alpes fr.

* B Crispata Koch. (A. crispata Willd.). - Cenisio al lago bianco (Bouvier).

A. saxatilis AlL. - Comune a Fossimagna ed intorno Susa e la Novalesa in siti aridi e sassosi. Trovasi pure presso Cesana.

Ann. Giugno, Luglio.

Arabetta rupina it. - Arab. des Rochers fr.

A. hirsuta Scop. (Turritis hirsuta RE-Lin.) - Nei muri e nei siti ombrosi dei colli.

Ann. Aprile, Giugno.

Baccellino it. - Arab. herissée fr.

A. auriculata LANK. (A. aspera Re-AlL.) - Nelle vigne presso Susa.

Ann. Aprile, Maggio.

Arabetta orecchiata it. - Arab. auriculée fr. 
* Foglie cauline semplici sessili talvolta amplessicaule, ma non cuoriformi, più o meno irsute.

* A. ciliata R. Brown. - Altipiano del Cenisio (Negri). Cenisio (Bonjean in Bertol.).

Bien. Giugno, Luglio. Arabetta cigliosa it. - Arab. ciliée fr:

* A. muralis Bertor. - Alle Blaccie e dintorni di Gravere (Negri).

Per. Maggio, Giugno.

Arabetta dei muri it. - Arab. des mus fr.

A. serpyllifolia Vilu. (A. scabra Re-AlL.) - Siti sassosi delle Blaccie e di Monpantero.

Per. Giugno, Luglio.

Arabetta pepolino it. - Arab, à feuilles de serpolet fr.

2. Semi cinti di un'ala larga membranosa.

* Foglie glabre intere o quasi.

A. caerulea HaEnK. (Turritis caerulea Re-Ald.) Presso le nevi in liquefazione: siti umidi ed elevati del Cenisio presso l'Eau blanche.

Per. Luglio, Agosto. (Foglie spesso cigliate).

Arabetta fioraliso it. - Arab. bleue fr.

Bella specie alpina. Ha i fiori cerulei.

Altipiano del Cenisio (Negri).

A. bellidifolia Jace. Lin. Mant. - Non rara nei luoghi umidi e freddi del Cenisio e nelle Alpi di Giaveno.

Per. Giugno, Luglio.

Arabetta lucida it. - Arab. à feuilles de paquerette fr. 
* Foglie peloso - dentate,

spesso intere nell' Arabis pumila.

* A. pumila JACQ. - Cenisio (Bouvier)

Per. Giugno, Luglio.

Arabetta stellata it. - Arab. naine fr.

A. turrita Lin. - Comune intorno Susa nei siti ombrosi e sassosi delle Blaccie e della Madonna della Consolata.

Bien. Aprile, Giugno. (Foglie cuoriformi amplessicaule). Cavolessa selvatica it. - Arab. tourette fr.

Gen. $6^{\circ}$ - Candamine LIn.

Semi uniseriati.

(Tutte le specie della Flora hanno fiori bianchi)

- Foglie semplici interé o quasi.

* C. asarifolia Lin. - Siti sassosi presso i torrenti alle falde del Cenisio specialmente sotto le Ferrere. Cochlearia d' montagna vern. (All. ped., I, p. 260).

Per. Giugno, Agosto.

Billeri rotondifoglio it. - Cardamine à feuilles d'Asaret fr.

Bella e distinta specie dimenticata dal Re. Presso i rivoli nelle Alpi di Condove.

(Foglie orbicolari, cuoriformi, sinuato-dentate).

C. alpina Wrrcd. (C. bellidifolia Re-Alc.) - Nel Cenisio all'Eau blanche: Alpi di Giaveno.

Per. Giugno, Agosto.

Billeri d'Alpe it. - Card. des Alpes fr.

Cenisio, cima di Ronche (Negri).

Graziosa pianticella alpina a foglie peziolate, ovato-ot. tuse. Le cauline talvolta trilobate.

Nel testo C. bellidif. Lin. dal sinon. di Allioni, specie diversa, svedese (Koch, Bertol.). 
* Foglie radicali infime semplici

le restanti pennate o pennate e ternate.

C. resedifolia Lis. - Freq. nelle rupi alpine.

Per. Giugno, Agosto.

Billeri pennato it. - Card. à feuilles de reseda fr.

Cenisio, cima di Ronche (Negri).

C. Plumierii VILL. (C. thalictroides RE-AlL.) - Siti montani di Foresto e del Cenisio. Luoghi sassosi ed ombrosi delle Alpi di Giaveno.

Bien. Maggio, Giugno. (Unghia dei petali gialla).

Billeri colombino it. - Faux pigamon fr.

Sagra di San Michele. (Arcangeli).

C. amara Lin. - Presso le fonti nei siti mon$\operatorname{tani}$ ed alpini.

Per. Maggio, Giugno.

Billeri amaro it. - Card. amère fr.

*** Foglie tutte pennate.

C. impatiens Lin. - Comune lungo i fossi e nei siti ombrosi delle selve; presso Susa lungo i fossi della via che mena a San Lazzaro.

Ann. Aprile, Giugno.

Billeri it. - Card. impatiente fr.

C. hirsuta Lis. (C. parviflora RE-ALL. dal sito) - Ovunque in primavera nei pascoli e sui muri.

Ann. Marzo, Aprile.

Billeri primaticcio it. - Card. velue fr.

Nel testo C. parviflora Lin. evidentemente dall'Allioni. C. parviflora Lin. è specie diversa che abita siti palustri. 


\section{Gen. ro $^{\circ}$ Dentaria Lin.}

(Fiori violacei)

D. pentaphyllos $\beta$ e y Lin. - D. minor Off. - Nella contrada di Fossimagna, ed in abbondanza sopra Bussolino. - Rimedio disusato.

Per. Aprile, Maggio.

Dentaria it. - Dentaire à feuilles digitées fr.

B) Cotiledoni incombenti. (Cot. incumbentes).

\section{Gen. $8^{\circ}$ - Hesperis Lin.}

(Stigma bilobato.)

H. matronalis LiN. - H. o Viola matronalis Off. - Presso la Dora al ponte di San Rocco ad occi. dente di Susa. Rimedio disusato.

Bien. Maggio, Giugno.

Esperide it. - Julienne des dames fr.

Fiori lilla, fragranti di sera.

\section{Gen. $9^{0}$ - Sisyambarienar Lin.}

* Fiori bianchi - Fogtie indivise.

S. alliaria Scop. (Erysimum alliaria Re-Lin.) - Alliaria Off. - Presso Susa in copia nei prati della Bretagna e di Croale. Acre, antiscorbutica.

Bien. Aprile, Maggio.

Alliaria it. - Alliaire fr.

Disusata come rimedio; le bestie la mangiano avidamente. Le foglie stropicciate danno odore di aglio.

S. Thalianum GAud. (Arabis. Thaliana Re-Lin.) - Comunissima nei siti macilenti.

Ann. Aprile, Maggio.

Pelosella it. - Sisymbre de Thalius fr. 
* Fiori gialli, foglie pennatifide o 3 pennate.

S. officinale Scop. (Erysimum officinale RE-LiN.) Ery. vulgare o Irio 0ff. - Frequente nei siti pingui, incolti. Acre, antiscorbutico.

Ann. Maggio, Settembre.

Erba cornacchia it. - Sisymbre fr.

Un giorno si usava contro i catarri polmonari cronici e l'abbassamento della voce in sciroppo o infuso. Rimedio per altrő non del tutto disusato in Francia ed in Svizzera.

* S. austriacum JACQ. - Cenisio a piedi delle mura dell' Ospizio (Bouvier).

Bienn. Maggio, Luglio.

Sisimbro attortigliato it. - Sysim. d'Autriche fr.

y) Acutangulum Koch (Sinapis pyrenaica RE-LIN. In copia presso le case sul Cenisio.

S. Irio Lin. - Comune nella Valle di Exilles e specialmente sulle rive della Dora ed a Susa nei luoghi incolti lungo le mura. Acre.

Ann. Aprile, Magigio.

Erba irida it. - Vélaret fr.

S. Sophia Liv. - Ovunque nei siti incolti e lungo le vie. Semi vermicidi.

Ann. Maggio, Luglio. (Foglie 3 pennate).

Erba Sofia it. - Sys. Sagesse - Sagesse des chirurgiens fr. Rimedio disusato. 


\section{Gen. 10 - Huguenimaa Reichn.}

H. tanacetifolia RetchNв, (Sisymbrium tanacetifolium Re-Lin.) - Copiosa nelle montagne di Chiomonte e del Cenisio.

Per. Luglio, Agosto.

Sisimbro zolfino it. - Hugueninie tanaisie fr.

Fiori gialli - Foglie pennatifide. Siliqua trinervata. Nel Gen. Sisymbrium la Siliqua è uninervata.

Gen. 11. - Braya Sternb. e Hopp.

B. pinnatifida Косн. (Sisymbrium bursifolium REVilu.) - Nei pascoli del Cenisio.

Per. Luglio,. Agosto.

Sisimbro ruculoso it. - Braya pinnatifide fr.

(Sysimbrium pinnatifidum DC. Sisym. dentatum All.)

Siliqua a semi biseriati - Fiori bianchi. - Graziosa pianticella alpina, come la Hugueninia. Nella Hugueninia e nel Sysimbrium i semi sono uniseriati.

Nel testo: S. bursifolium Lin. specie diversa affatto, non alpestre.

\section{GeN. 12. - Erysimuma.}

(Fiori gialli)

- Pedicilli due volte pit corti del calice.

E. pumilum Gaud. (Cheiranthus alpinus Re-LiN.) Non raro all'Assietta ed al Cenisio.

Per. Luglio, Agosto.

Crespinaccio d'Alpe it. - Vélar nain fr.

Colle delle Finestre.

Ch. alpinus All. non Lin. sec. Bertol. (Fl. it. Vol. VII, p. 89.)

* E. rheticum DC. - Sulla Brunetta (Negri).

Per. Maggio, Giugno.

Crespinaccio Retico it. - V. de Rhétie fr. 
- Pedicilli lunghi press'a poco quanto il calice.

E. canescens Roth. (Cheiranthus Boccone Re-AlL.) - Frequente nei pascoli aridi a Susa e nei siti aprici delle Blaccie.

Ann. Giugno, Luglio.

Crespinaccio giallo it. - Vélar canescent fr.

C) - Cotiledoni raddoppiati. (Cot. conduplicatae).

Gen. 13. - Brassiea Lin.

(Fiori gialli).

B. Richerii ALL. - Nei gioghi altissimi di Fossimagna e del Vallon. In copia specialmente al piccolo Cenisio nel luogo detto Comba d'Ambin.

Per. Luglio.

Brassica bislunga it. - Chou de Richer fr.

Bella pianta alpina a fiori disposti in lunghi grappoli.

B. repanda. DC. (Sysimbrium repandum RE-WILLD.) - In copia nella valle di Bardonêche e nelle località alte e sassose tra Foresto e Bussolino.

Per. Giugno, Luglio.

Brassica carnosa it. - Chou sinué fr.

* B. oleracea Lin.

Bien. Mag. Giugno.

Cavolo it. - Chou potager fr.

Originaria dei nostri littorali; coltivata, e profondamente modificata dalla coltura presenta moltissime varietà e sottovarietà, fra le quali noteremo;

Il cavolo, Coi vern. suddiviso in molte sottovarietà. (Br. oleracea capitata Lin.) Il Cavolfiore, Cavolifior vern. (Br. oleracea Botrytis Lin.) è una sotto varietà di esso, il Broccolo, Broccole vern. 
* B. napus Lin.

Ann. o bienn. Aprile, Giugno.

Ravizzone it. - Chou Navet, Navet fr.

Dai semi di questa pianta si estrae un olio buono per ardere e per condire alimenti.

\section{* B. rapa LIN.}

Ann.-bien. Aprile, Giugno.

A) Oleifera. - Colza it. - Navette fr.

Dai semi si estrae un olio simile a quello del Ravizzone. $B$ ) Esculenta. - Rapa it. - Rava vern. - Rave fr. Coltivata per le sue radici carnose come alimento.

Gen. 14. - Erueastaum Schimper e Spenner.

E. obtusangulum RercbH (Brassica erucastrum ReArr.) - Nelle vie, nei luoghi sabbiosi presso le acque e nei pascoli.

Ann. Aprile, Maggio.

Brassica rucola it. - Erucastrum à angle obtus fr.

Ha fiori gialli; le foglie pennatifide.

Nel testo Br. Erucastrum Lin. dal sinonimo di Allioni; specie diversa come ben dimostra il Bertol. (Fl. it. Vol. VII, pag. 159). Vedi anche Kioch. Syn. ed. 3a, pag. 49.

* Gen. 15. - Diplotaxis DC.

D. tenuifolia DC. - Presso la Cappella di Ean Grato, passato il Castello di Rivoli; sulla Brunetta. Per. Aprile, Ottobre. (Fiori citrini).

Ruchetta selvatica it. - Diplotaxie à feuilles menues fr.

Gen. 16. - Simapis LIN.

S. arvensis Lin. - Freq. nei campi e nei luoghi pingui.

Ann. Maggio, Luglio. (Fiori gialli).

Rapaccini it. - Moutarde des champs fr. 
S. Tournefortii AlL. - Raccolta da Ignazio Molineri nei siti elevati presso il torrente di Fossimagna.

Per. Luglio.

Brassica pennata it. - Moutarde giroflè fr.

(Brassica cheiranthus DC. - Sinapis cheiranthus Koch.) Fiori giallo-pallidi grandi, foglie pennatifide. - Cenisio (Bonjean in Bertol.)

La senapa medicinale tanto nota è propriamente Brassica Nigra Koch. (Sinapis Nigra Lin.).

\section{* Gen. 1\%. - Eruca DC.}

\section{E. sativa DC. - Coltivata.}

Ann. Maggio, Settembre.

Eruca, ruchetta it. - Aruga vern. - Roquette fr.

Notissima come gustoso alimento in insalata.

\section{Sezione $2^{a}$ - Siliculose: Siliqua breve,}

\section{lunga presso a poco quanto larga (\$ilicula).}

1) Silicula bivalve deiscente, compressa pit o meno dal dorsn o piana.

A) Cotiledoni accombenti.

\section{Gen. 18. - Veseicaria LAMK.}

V. utricolata. Lamk. (Alyssum utriculatum. Re LiN. Mant. ) - Trovasi in copia nelle rupi presso Susa, specialmente delle Blaccie: la vidi anche nei siti aprici di Condove nella contrada detta la Mura.

Per. Maggio, Giugno. (Fiori gialli).

Alisso otricellato it. - Vescicaire renflée fr.

Foglie interissime. Silicula globosa a stile lunghissimo. 


\section{Gen. 19. - lyssum LIN.}

(Fiori gialli : filamenti pit o meno appendiculati)

* Cellette della Silicula contenenti ciascuna un solo seme.

A. alpestre Lin. Mant. - Sommità dell' Assietta e del Cenisio.

Per. Luglio, Agosto.

Alisso alpestre it. - Alysson des Alpes fr.

A. argenteum Willd. (Lunaria argentea RE-ALL.). - Trovato da me nei monti di Bussolino verso la fabbrica del marmo e da Ignazio Molineri nei monti di Caselette, nè manca a S. Michele della Chiusa.

Per. Aprile, Maggio.

Alisso del gabro it. - Alys. argenté fr.

È pascolo molto grato alle pecore.

Abbonda sul Musinè (Re, Fl. Tor.).

* " Cellette della Silicula contenenti ciascuna due semi.

A. montanum, Lis. - Luoghi montani di Monpantero; Cenisio.

Per. Maggio, Giugno.

Alisso montanino it. - Alys. des montagnes fr.

Cima di Ronche al Cenisio (Negri).

A. calycinum Lin. - Nei campi e nei pascoli sabbiosi. Alle Blaccie presso Susa.

Ann. Maggio, Giugno.

Alisso calicino it. - Alys. des montagnes fr. 


\section{Gen. 20. - Lunaria Lin.}

L. rediviva Lin. - Dirupi di S. Michele della Chiusa; presso la fabbrica del marmo ed i laghi d'Avigliana.

Per. Maggio, Giugno.

Lunaria odorosa it. - Lunaire vivace fr.

Fiori violacei fragranti, silicula grande.

\section{Gen. 21. - Petrocallis R. Brown.}

P. pyrenaica Brown. (Draba pyrenaica Re-LiN.). Culmini delle Alpi all'Assietta, al Vallon, al Cenisio.

Per. Giugno, Luglio.

Pelosella palmata it. - Petrocallis des Pyrenées fr.

Graziosa pianticella alpina. Fiori rosei o pallido-violacei: foglie cuneato-trifide cigliate.

\section{GEN. 22. - Draba LiN.}

" Fiori gialli.

D. aizoides Lin. - Nei pascoli delle vette alpine.

Per. Maggio, Giugno.

Aizoide it. - Drave faux aïzoon fr.

Caule nudo, glabro. - Foglie lineari rigide cigliate. Elegante pianticella alpina. Cima di Ronche (Negri).

* D. nemoralis Willd. - Sopra Bussolino a Balmaforte (Balbis in Bertol.).

Ann. Aprile, Giugno.

Draba boschereccia it. - Drave des bois fr.

(Draba nemorosa Lin. Spec. Pl. Ed. $1^{2}$ ). 
* D. Johannis Hosr. - Cenisio all' Eau blanche (Negri) (Bonjean in Bertol.).

Per. Luglio, Agosto.

Pelosella barbellata it. - Drave des neiges fr. (Draba nivalis Willd. Balbis Misce bot. $1^{2}$ ).

D. frigida Saut. (D. hirta Re-Alz.) - Sul Cenisio. Per. Giugno, Luglio.

Pelosella spuntata it. - Drave des frimas fr.

Nel testo Draba hirta Lin. dal sinonimo dell'Allioni. Draba hirta Lin. è pianta Lapponica.

D. verna LiN. - Dovunque nei siti aridi e nei pascoli sterili.

Ann. Marzo, Aprile.

Pelosella it. - Drave printanière fr.

\section{Gen. 23. - Coelnleania LIN.}

c. saxatilis Lamk. (Myagrum saxatile Re-LiN.) Abbonda nelle alte rupi presso il Vallon nella valle d'Exilles, e nei siti sassosi di Fossimagna.

Per. Giugno, Luglio (Fiori bianchi).

Cochlearia alpina it. - Cochléaria des rochers fi.

Comba di Meana nei siti umidi delle Traverse.

B) Cotiledoni incombenti.

Gen. 24. - Camelima Crantz.

G. Sativa Crantz. (Myagrum sativum, Re-LiN.) Myagrum o Chamaelina Off. - Frequente nei campi.

Ann. Maggio, Luglio.

Camelina it. - Cameline cultivée fr.

Dai semi della Camelina coltivata si estrae circa il 300,0 
di olio buoilo per lampade e per condire vivande nei siti dove manca l'olio d'olivo. Erano i semi della Camelina tenuti una volta come purgativi, oggi sono caduti in disuso.

2) Silicula bivalve deiscente, riu o meno compressa dai lati; valve piegate a forma di navicella.

A) Cotiledoni accombenti.

Gen. 25. - Thlaspi LIN.

1. Silicula largamente alata.

- Stilo sub-nullo.

T. arvense LIN. - Nei campi.

Ann. Maggio, Giugno. (Fiori bianchi).

Erba storna it. - Tabouret des champs - Herbe aux écus fr.

T. perfoliatum LiN. - B. Th. perfoliatum minus, Baun. Pinax 106. - Nei pascoli. La varietà B fu da me trovata nelle vigne delle Blaccie presso Susa.

Ann. Aprile, Maggio. (Fiori bianchi).

Erba montanella it. - Tab. perfolié fr.

* Stilo più o meno lungo.

T. alpustre Lrv. - Abbonda nei prati del Cenisio e nelle Alpi d'Exilles.

Bienn. Giugno, Luglio. (Fiori bianchi o bianco-rosei).

Tlaspi azzurrognolo it. - Tab. alpestre fr.

T. montanum Lin. - Raccolto da Ignazio Molineri nella contrada di Fossimagna ed a Caselette.

Per. Maggio, Giugno. (Fiori bianchi).

Tlaspi montanino it. - Tab. des montagnes fr.

Cenisio, Rocciamelone (Bonjean, De TVelden in Bertol.). 
2. Silicula cinta di ala angustissima.

* T. rotundifolium Gaud. - Cima di Ronche (Negri). - Cenisio (Bonjean in Bertol).

Per. Luglio, Agosto. (Fiori violacei).

Iberidella it. - Tab. à feuilles rondes fr.

Bella pianta alpina che vegeta su parecchie cime delle alpi Piemontesi. (Hutchinsia rotundifolia DC.)

GEN. 26. - Bberis LiN.

(Petali ineguali - Silicula alata).

I. saxatilis LIN. - Trovata da me in copia nelle rupi di Foresto presso la Cateratta. Trovasi pure presso i torrenti ad Exilles.

Per. Maggio, Giugno. (Fiori bianchi).

Tlaspi rupino it. - Ibéride des rochers fr.

1. pinnata LiN. - Frequente nei campi.

Ann. Giugno, Luglio. (Fiori bianchi, talvolta violacei).

Caspi it. - Ibéride à feuilles pennées fr.

\section{GEN. 2\%. - Miseutella LIN.}

(Fiori gialli)

- Sepali esterni cornicolati.

B. auricolata LIN. - Siti sassosi sopra Foresto. Ann. Maggio.

Biscutella beccuta it. - Lunetière à oreillettes fr.

- Sepali esterni non cornicolati, uniformi agli interni.

B. laevigata LiN. Mant. - Nei prati alpini macilenti. 
Per. Maggio, Giugno.

Biscutella montanina it. - Lunetière lisse fr.

ß. Coronopifolia. Foglie ispide profondamente dentate ( $\boldsymbol{B}$. Coronopifolia RE-ALL.). Raccolta da Ignazio Molineri nelle alpi altissime di Cesana.

Dintorni di Cesana (De-Filippi)

Nel testo: B. Coronopifolia Lin. dall'All. e dal Willd.

Abbiamo potuto riscontrare, mercè la gentilezza del prof. Arcangeli, gli esemplari dell'erbario di Allioni, i quali però non concordano molto con la varieti $\beta$ del Bertoloni, alla quale questi rapporta B. Coronopifolia All. (Fl. it., vol, VI, pag. 526).

B) Cotiledoni incombenti.

GEN. 28. - Lepidinaren LIN.

(Fiori bianchi: un seme per celletta).

* Silicula sprovvista di ala o appena alata all'apice.

\section{Iatifolium Lis. - L. vulgare - Lepidium Off. -} Abbonda presso la Dora a Piazza Savoia attigua a Susa. Acre.

Per. Giugno, Agosto.

Erba mostardina it. - Passérage à larges feuilles fr. Antico farmaco stimolante antiscorbutico.

\section{L. ruderale Lin. - Presso le case.}

Ann. Aprile, Maggio. (Silicula brev. alata all'apice). Lepidio dei calcinacoi it. - Pass. des décombres fr.

I. graminifolium LiN. (L. Iberis $\mathrm{RE}_{\mathrm{E}} \mathrm{DC}$. syst. nat.) Iberis cardamantica ovvero lberis Off. - Abbonda nei pascoli e presso le mura nei luoghi secchi. Acre, diuretica. 
Per. Giugno, Settembre.

Erba da sciatica it. - Pass. graminée fr.

Rimedis disusato.

$\mathrm{Nel}$ testo L. Iberis Lin. confuso da parecehi autori col vero L. graminifolium Lin., il quale ha costantemente sci stami non due come L. Iberis. (Vedi Bertol, Fl it., vol. VI, pag. 584 e Kock Syn., ed. $3^{\text {a }}$, pag. 63).

\section{* Silicula alata.}

I. campestre R. Brown (Thlaspi osmpesire Re-liN.) - Presso i campi.

Ann. Aprile, Maggio.

Erba storna it. - Pass. champêtre fr.

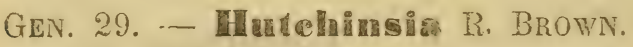

(Fiori bianchi).

F. alpina R. Brown (Lepidium alpium Re-lsN.) -Volgare nei luoghi alpini freddi ed umidi.

Per. Luglio, Agosto.

Iberidella alpina it. - Ilutchinsic des rocailles fr.

Caule nudo - Foglie pennatipartite.

A Mustion salendo da Villarfocchiardo al piano delle $\mathrm{Ca}$ valle (Bruno).

E. petraea R. Brown (Lepiclium petracum Re-LIN.) - Comunissimo nei siti aridi presso Susa.

Ann. Maggio.

Iberidella rupina it. - Hut. des pierres fr.

\section{GEn. 30. - Capsella Midis.}

\section{c. bursa pasioris Moexch (Thlaspi burse pesion's RE-} LIN.),

Per. Marzo, Settembre.

Borsa di pastore it. - Capselle bourse a pasteur fr. 
GeN. 31. - Aethiomema R. BRown.

A. saxatile R. Brown (Thlaspi saxatile RE-LIN.) - Ovunque nei luoghi sassosi presso Susa e presso le vigne a Monpantero.

Ann. Aprile, Maggio. (Fiori rosei).

Thlaspi carnicino it. - Ethionème des rochers fr.

Sagra di S. Michele (De-Filippi).

Sezione $3^{*}-$ Silicula indeiscente biloculare, - spesse, per distruzione del tramezzo, uniloculare.

A) Cotiledoni incombenti.

GEN. 32. - Isatis LIN.

I. tinctoria Lis. - Isatis o Glastum Off. - Abbonda presso Susa nelle località dette dei Pusin e di S. Martino. Comune ancora nei monti di Giaveno ed intorno al tempio di San Michele della Chiusa. Le foglie danno un colore ceruleo.

Bienn. Aprile, Maggio.

Guado it. - Pastel fr.

Musinè, lato che guarda la Dora. (Re, Fl. Tor.)

Le foglie dell' Indigofera tinctoria Lin. e di altre specie consorelle, che ci provengono da varie regioni tropicali, hanno fatto abbandonare quasi del tutto la coltura del Guado per l'industria tintoria.

\section{Gen. 33. - Myagram LiN.}

\section{M. perfoliatum LiN. - Nei campi.}

Bienn. Maggio, Giugno.

Miagro liscio it. - Myagre fr. 
Fiori gialli - Silicula a tre cellette; le due superiori parallele vuote.

\section{Gen. 34. - Neslia Desv.}

N. paniculata Desv. (Myagrum paniculatum RE-LiN.). Ann. Maggio, Giugno.

Miagro pannocchiuto it. - Neslie fr.

Fiori giallo-pallidi. Silicula globosa ad una celletta contenente un seme.

B) Cotiledoni raddoppiati.

\section{GeN. 35. - CaRepima Desv.}

C. corvini Desv. (Crambe Corvini Re-All.) - Comunissima nelle vigne presso Susa.

Ann. Maggio, Giugno.

Miagro stellato it. - Calepine de Corvin fr.

Fiori bianchi con unghia gialliccia.

C) Cotiledoni lineari ripiegati trasversalmente.

(Cot. biplicatae).

Gen. 36. - Semebiera Pers.

S. coronopus Porret (Cochlearia coronopus RE-LiN. - Ambrosia campestris Off.) - Presso le case. Acre. Ann. Giugno, Agosto.

Lappolina it. - Senebière corne de cerf fr.

Fiori bianchi - Silicula a forma di cresta a valve rugose biloculare.

Rimedio disusato. 
D) Cotiledoni lineari ravvolti a spirale.

(Cot. spirales).

GeN. 37. - Bunias LiN.

\section{B. erucago LiN. - Nelle messi.}

Ann. Luglio. (Fiori gialli).

Cascellare it. - Bunias fausse roquette fr.

In molte parti del mezzogiorno d'Italia il Bunias erucago costituisce una vera risorsa alimentaria per la gente povera. Sono mangiate le foglie condite con olio.

\section{Sezione ${ }^{2}$ - Siliçมа o Silicala articolata;}

ogmi articolo contenente wn seme.

$$
\text { (Cotiledoni raddoppiati). }
$$

- Siliquose.

\section{GEN. 38, - Remphamus LIN.}

R. raphanisîrum LIN. - Nelle messi.

Ann. Giugno, Luglio. (Petali bianchicci o violetti). Rafanistro it. - Ravenelle fr.

* R. sativus Lin. - Coltivato.

Ravanin vern. - Ravanello it. - Radis fr.

Presenta molte varietà, tutte coltivate.

- Siliculose.

Gen. 39. - Bapistum BoerH.

R. rugosum ALL. (開yagrum rugosum RE-LIN.) Nelle messi.

Ann. Maggio, Giugno. (Fiori gialli).

Miagro peloso it. - Rapistre ridè fr. 
* Ordine VII. - Capparidee (Capparidece Juss.). Gen. Unico - Camparis Lin.

C. spinosa Lin. - Castello di Caselette (Re, App. $1^{a}$ ad Fl. Ped.). Cimitero di Rivoli (De Filippi).

Suffrut. Giugno, Luglio.

Cappero it. - Caprier épineux fr.

Il Cappero spinoso è coltivato nella Francia meridionale e specialmente a Tolone; le sue gemme fiorifere vengono colte e preparate all'aceto e sono oggetto di lucroso commercio. Crediamo peró che esso non sia spontaneo nel territorio di Susa e che vegeti nelle localiti sopra accennate od in altre per mero incidente.

\section{Ordine VIII. - Cistinee (Cistinece DC.) \\ Gen. $1^{0}$. - Cistus Lin.}

C. salvifolius LIN. - Abbonda nelle rupi aride di Condove.

Suffrut. Aprile, Maggio. (Fiori bianchi - Foglie ovate). Cisto it. - Ciste à feuilles de sauge fr.

Gen. 2. - Hellamthemum Tournf.

1) - Foglie, o tutte, o le inferiori opposte stipulate: eccetto nell' $H$. italicum.

* Fiori gialli.

H. vulgare Gagrtn. (Cistus helianthemum Re-Lin.) Helianthemum 0ff. - In tutti i pascoli sterili. Farmaco disusato.

Per. Giugno, Luglio.

Eliantemo it. - Helianthème commun fr.

* $\beta$. hirsutum Koch - Helianthemum obscurum Pers. Dintorni di Susa (Re, app. alt. ad Fl. ped.). Cenisio (Bonjean in Bertol.). 
๖. Grandiflorum Косн. (Cistus grandiflorus RE-ALL.). - Frequente nelle Alpi.

* H. croceum Bertol., Fl. it. in parte. (H. hirtum RE, Fl. Tor., vol. $1^{\circ}$, pag. 281). (H. vulgare Parlat. Fl. it.) - Musinè.

Per. Maggio, Luglio.

H. italicum Pers. (Cistus alpestris Re-Ald.) - Nei siti montani ed alpini. Discende in pianura ed abbonda ad Oriente della Brunetta e nelle vigne di Monpantero.

Per. Giugno, Agosto.

Eliantemo rupino it. - Heliant. d'Italie fr.

Assietta.

H. guttatum MrLl. (Cistus guttatus Re-LiN.) - Nei pascoli secchi, sterili.

Ann. Giugno, Agosto.

Fior gallinaccio it. - Heliant. tachè fr.

H. glutinosum Pers. (Cistus glutinosus Re. Lin. Mant.). - Fra i sassi presso le vigne di Monpantero e nelle rupi di Foresto.

Per. Maggio.

Eliantemo peverella it. - Heliant. glutineux fr.

È da notarsi che questa specie è propria dei litorali marini Tirreni, delle nostre isole, ed in generale delle latitudini calde della penisola.

* Fiori bianchi.

H. polifolium DC. (Cistus pilosus RE-Alc.) - Abbonda nei colli solegrgiati da Susa a Borgone.

Per. Maggio, Luglio.

Eliantemo Canutola it. - Heliant. poilu fr.

A Monpantero (Parlat. Fl. it.). 
Nel testo C. pilosus Lin. dall Allioni che ritenne per una specie sola Cistus pilosus e polifolius Lin. (Ped., 2, p. 105).

2) Foglie sparse senza stipole. Fiori gialli.

H. fumana MrLl. (Cistus fumana Re-Lin.). - Volgare nei siti aprici dei colli soleggiati. Abbonda a Condove nei siti sterili della contrada detta Bialera della Reisia.

Per. Giugno, Luglio.

Fumana it. - Heliant. à feuilles menues fr.

Musinè (Re, Fl. Tor.)

Ordine IX. - Violacee (Violacece Vent.).

Gen. UnICO - Violla Lin.

1. I due petali intermedii pressochè orizzontali.

A) Acauli.

- Foglie subintere, al pit crenate, reniformi o cuoriformi.

V. hirta Lin. - Nei prati macilenti.

Per. Marzo, Aprile.

Violetta senza odore it. - Violette herissée fr.

Viola odorata LiN. - Viola martia Off. - Violette vern. - Abbonda nei siti pingui incolti. Radice emetica diuretica ed i fiori in infusione, emollienti.

Per. Marzo, Aprile.

Mammola it. - Violette odorante - Fleur de mars fr.

I fiori, e sopratutto le radici della V. odorata, sono emetici e non abbandonati dalla farmacia moderna.

* Viola palustris Lin. - Cenisio (Bouvier). Piccolo Cenisio alla Combe des Cevinnes (Bonjean in Bertol.).

Per. Maggio, Giugno.

Violetta palustre it. - Violette des marais fr. 
* Foglie partite a lacinie - 2 - 3 dentate.

Viola pinnata Lis. - Abita i monti di Cesana ed i gioghi dell' Assietta fra Albergian e Fenestrelle. Trovasi pure al Cenisio, ma è rara.

Per. Giugno, Luglio.

Violetta ventaglina it. - Violette decoupée fr.

\section{B) - Caulescenti.}

(Foglie sub-intere al pit crenate o seghettate).

Viola canina Lin. - Intorno alle selve nei siti montani e collinosi. Radice emetica, catartica. Cenisio (Bonjean in Bertol.).

Per. Aprile, Giugno.

Viola mammola senza odore it. - Violette canine fr.

Rimedio disusato.

\section{Viola montana Lin. - Nelle selre.}

Per. Maggio.

Viola arborea it. - Violette des chiens fi.

Viola Allionii Pro (Viola balbis RE.) - Raccolta da me sul Cenisio, ma è rara. Cauli prostrati, foglie orbicolari crenate piuttosto consistenti.

Questa specie di viola fu già notata dal Chiar. Allioni nel suo Auctariúm ad Fl. Pedem., pag. 29, nel quale ci lasciò di essa un' ampia descrizione, ma non osò proporla come specie nuova non avendo ancora la coltura confermati i suoi caratteri. Su di ciò consultai l'esimio botanico Ignazio Molineri e da questi fui assicurato che questa specie di viola da molti anni coltivata non mutò giammai il suo abito; non dubito punto per conseguenza 
che si possa ritenere come specie distinta. E volontieri colgo quest'occasione per dedicarla al $\mathrm{Ch}$. prof. di botanica Balbis a testimonianza della mia gratitudine verso quest'illustre personaggio.

Per. Maggio, Giugno.

Mammoletta scabra it. - Violette des sables fr.

Come apparisce dalla nota precedente il Re diede a questa specie il nome di Viola Balbis. Noi abbiamo stimato di segnarla col nome di V. Allionii, ad essa dato con [maggior ragione da G. B. Pio.

Cenisio (Bouvier) (Bonjean in Bertol.).

\section{Viola mirabilis LIN. - Nelle selve montane.}

Per. Aprile, Maggio.

Viola magica it. - Violette singulière fr.

Peduncoli radicali misti a caulini.

\section{I due petali intermedi}

rivolti in su e coprenti $\mathrm{i}$ due superiori.

* Petali gialli, l'inferiore striato di rosso cupo.

Viola biflora Lin. Frequentissima nei luoghi ombrosi ed umidetti delle Alpi.

Per. Maggio, Giugno.

Viola gialla it. - Violette à deux fleurs fr.

Graziosissima pianticella alpina.

* Petali variegati, bianco-azzurro-gialli.

Viola tricolor Lin. - Ovunque nei campi. Rimedio per la crosta lattea.

Ann. Maggio, Giugno.

Viola a tre colori it. - Pensé vern. - Pensèe fr.

Non ancora disusato come farmaco emetico e purgativo e anche per l'uso sovraccennato dal Re. 
La V. tricolor ha risposto mirabilmente alla coltura, la sua corolla assunse per essa grandi proporzioni e si vesti di colori splendidi e gentili.

* * * Petali violetti con sfumatura gialla alla base del petalo speronato o ceruleo violetti.

\section{Viola calcarata Lrn. - Prati e pascoli alpini.}

Per. Luglio, Agosto.

Pensieri odorosi it. - Violette à long éperon fi.

Bellissima specie a fiori grandi di un odore poco intenso ma soavissimo.

* B. lutea Kock (Viola Zoysii TVuif) - Corolla gialla Cenisio (Bouvier). Per lo più mista alla specie.

Viola cenisia Lin. - Luoghi sassosi elevati del Cenisio e copiosa specialmente al sito detto Ronche.

Per. Luglio, Agosto.

Mammola rupina it. - Violette du mont Cenis fr.

Bella specie anch'essa; ha tutte lo foglie interissime, la corolla ceruleo-violetta.

Ordine X. - Resedacee (Resedacea DC.)

\section{GEN. UNICO - MReseda LIN.}

Reseda lutea Lin. - Nelle rupi presso Susa.

Ann. Giugno, Agosto (Fiori giallicci).

Guaderella cruciata it. - Réséda jaune fr.

R. phyîeuma Lin. - Abbonda nelle vigne. Ann. Giugno, Agosto. (Fiori bianchi). Reseda selvatica it. $-R$. raiponce fr. La specie coltivata per la sua fragranza delicatissima. (Amorino it. Reseda odorata Lin.) è originaria dell' Egitto. 


\section{Ordine XI. - Droseracee (Droserucece DC.).}

\section{GEN. $1^{\circ}$ - Drosera LIN.}

(Fiori bianchi).

D. rotundifolia Lis. - Ros. o Rorella Off. - Tro. vata da Ignazio Molineri nei siti paludosi sopra Coazze. Acre, corrodente.

Per. Giugno. Agosto.

Rosolida it. -- Rossolis à feuilles rondes. Rosée du soleil fr.

\section{Iongifolia Lin. - Ros solis o Rorella longifolia} Off. - Virtù della precedente. Copiosa nelle paludi dei laghi d'Avigliana.

Per. Giugno, Agosto.

Rosolida lunga it. - Rossolis à longues feuilles fr.

Nei prati umidi in val della Torre, sotto il Dubion col nome di Drosera Anglica - Hudson. (Re, Fl. Tor.).

Rimedio disusato.

La Drosera rotundifolia e longifolia presenta un fenomeno molto interessante. Le sue foglie sono provviste nella pagina superiore e nel contorno di peli rossicci glandulosi, dai quali emana un liquido attaccaticcio; l' insetto imprudente, che cerca d'invaderla vi rimane impigliato. I peli che nello stato normale hanno l'apice curvo da dentro in fuori e che tendono con tutta la foglia a piegarsi, in presenza dell'insetto, da fuori in dentro, favoriscono l'entrata, e impediscono mirabilmente la fuga del prigioniero, che in poco tempo rimane ucciso e consumato, come se fosse divorato dalla foglia. Questo fenomeno singolare ha richiamato l'attenzione di uno dei più grandi naturalisti viventi, Carlo Darwin, il quale opina che tanto la Drosera quanto la Utricularia, la Pinguieula, piante nostre, ed alcune consorelle esotiche, siano addirittura carnivore. In queste pagine noi non possiamo 
nemmeno riassumere le accurate esperienze del Darwin, notiamo solamente che per quanto questa opinione possa essere non ancora pienamente ritenuta come fatto assolutamente assodato, avendo essa valenti oppositori, pur tuttavia non si può passare su di essa coll' indifferenza, colla quale la tratta il botanico Bellynck della Compagnia di Gesù, che scrive: "Nous ne perdrons pas notre temps à discuter de semblables balivernes ".

\section{Gen. $2^{\circ}$ - Parmagsi̊a LIN.}

P. palustris LIN. - P. o hepatica alba Off. - In tutti i prati e pascoli montani ed alpini umidi. Americante, sub-astringente.

Per. Giugno, Settembre. (Fiore bianco grande).

Parnassia it. - Parnassie, Gazon du Parnasse fr.

Rimedio disusato.

Ordine XII. - Polygalacee - (l'olygatece Juss.).

Gen. Unico - Polygala Lin.

- Erbacee.

P. vulgaris LiN. e Off. - Nei prati e nei pascoli ovunque. Radice amara.

Per. Maggio, Giugno.

Bozzolina it. - Bruttairole rousse vern. - Polygala commune fr.

P. amara Lrn. - Nel Cenisio e nelle Alpi d'Exilles. Radice e foglie amare stomatiche.

Per. Giugno, Agosto.

Bozzolina amara it. - - Polyg. amère fr.

La riputazione medicinale di espettorante tonica di queste specie di Poligale indigene è stata ecclissata dalla virtù della 
P. Senega Lin. comunomente detta Pcligala Virginiana dell'America nordica, di gran lunga piu di esse efícace. Nondimeno crediamo, che alla gente che abita le campagne priva di mezzi. le specie nostre e particolarmente la P. amara possono essere sodilisfacenti succedanee dell'esotica.

$$
\text { * Suffruticosa }
$$

P. Chamaebuxus Liv. -- Ovunque nelle selve presso Susa e nella contrada di Fossimagna.

Aprile, Giugno.

Bozzolina fruticolosa it. - Polyg. faux buis fr.

\section{Ordine XIII. - Silenee (SiTeneC DC.)}

A) Calice munito di squame alla base (Calice caliculato). Stili 2.

\section{GEN. $1^{0}$ - Diganthe LIN.}

- Fiori in apitoli o in fascicoli - Petali dentati o quasi interi. Foglie lineari. Fiori carnicini o purpurei.

D. carthusianorum LrN. - In quasi tutti i pascoli dei Colli asciutti. A Susa nelle rupi delle Blaccie.

Per. Giugno, Settembre. (Cenisio, Bonjean in Bertol.).

Garofanini da prato it. - Oeiilet des chartreux fr.

B. atrorubens DC. Prod. (D. atrorubens Re-All. - Pascoli e prati macilenti.

D. armeria Lis. -- Nei siti sabbiosi lungo le vie. Bienn. Giugno, Agosto.

Armeria it. - Oeillet veiu fr.

D. prolifer Lin. - Nei pascoli sterili.

Ann. Giugno, Luglio.

Strigoli it. - Oeillet prolifère fr. 
* Fiori solitari o in pannocchia.

a) Petali dentati o quasi interi.

D. neglectus Lors. (D. alpinus RE ALL. DC. Fl. Fr.). - Frequentissimo nelle alpi.

Per. Luglio, Agosto.

Viola dei monti it. - Oeillet négligé fr.

Cenisio (Negri) (Bonjean in Bertol.) Assietta. Falde dello Chaberton.

Nel testo: D. alpinus Lin. dal sinonimo dell'Allioni, specie diversa, se ben ricordiamo, non italiana.

D. sylvestris Wulf (D. caryophyllus RE a LIN.) - 〔ar. hortensis o Tunica Oif. - Nelle rupi della Brunetta.

I petali sono antispasmodici.

Per. Giugno, Agosto.

Garofanino scempio it. - Garofo vern. - Oeillet sauvage $f r$.

Farmaco disusato, almeno in Italia.

a Koch. Caule piu alto a 230 molti fiori. (D. virgineus RE-JACQ.) - Nei pascoli montani.

Nel testo: D. virgineus Lin. dal sinonimo di Allioni.

Dal D. caryophyllus $\alpha$ Lin. probabilmente derivarono le numerose varietà di garofani coltivati.

b) Petali pennatifido-multifidi.

D. superbus Lin. (D. plumarius Re-All.) - Da me trovato nelle alpi di Mocchie nella contrada detta la Portia e da Ignazio Molineri nelle alpi di Giaveno.

Per. Maggio, Giugno. (Fiori fragranti).

Garofanini it. - Oeillet superbe fr.

Nel testo: D. plumarius Lin. dal sinonimo di Allioni. 


\section{Gen. $2^{\circ}$ - Thanea Scop.}

T. saxifraga Scop. (Gypsophila saxifraga Re-Lis.) Nei pascoli sassosi e sterili.

Per. Giugno, Agosto.

Garofanina spaccasassi it. - Oeillet saxifrage fr.

B) Calice sprovvisto di squame alla. base (Calice non caliculato).

1) \$(ili 2.

Gen. $3^{\circ}$ - Gypsoplioila Lin.

G. repens Liv. (G. prostrata RE-ALL.). - In tutti i pascoli montani ed alpini.

Per. Luglio, Agosto.

Garofanina sdraiata it. - Gypsophile rampante fr.

Nel testo: G. prostrata Lin. dal sinonimo di Allioni, specie diversa, non europea.

G. muralis LiN. - Nei campi dopo la messe. Ann. Giugno, Settembre.

Garofanina minuta it. - Gyps. des murs fr.

Gen. $4^{\circ}$ - Saponaำ LIx.

- Calice angoloso-alato

Petali rosei sprovvisti di squame.

S. vaccaria LiN. - Abbonda nei campi.

Ann. Giugno, Luglio.

Strigolo it. - Saponaire des vaches fr.

* Calice cilindrico.

Petali provvisti ognuno di due squame.

Fiori rosei : gialli nella $S$. Lutea.

S. officinalis Lin. - Saponaria Off. - Frequente nei pascoli sabbiosi, pingui e presso le mura. Diuretica. 
Per. Luglio, Agosto.

Saponaria it. - Saponaire officinale fr.

La radice di questa pianta, quando è strofinata nell'acqua, fa spuma e scioglie i corpi grassi. Taluni in campagna se ne servono per nettare le stoffe di lana. La spuma è dovuta ad un principio acre che essa contione, la saponina.

Come farmaco, nei reumatismi e nelle malattie della pelle non è ancora del tutto disusato.

S. ocymoides LiN. - Volgare lungo la Dora nei siti incolti, nei pascoli e presso le vigne.

Per. Maggio, Agosto.

Ocimoide rossa it. - Saponaire basilic fr.

S. lutea Lrn. - Luoghi sassosi e freddi del Cenisio - Alpi di Giaglione - Assietta.

Per. Luglio, Agosto.

Saponaria gialla it. - Saponaire jaune fr.

Graziosa pianticella alpina, cespugliosa, foglie lineari glabre.

\section{æ) Stili æ.}

\section{Gen. $5^{\circ}$ - Cuerubalus Lin.}

C. bacciferus Lin. - Ovunque nelle siepi.

Per. Luglio, Agosto.

Baccolo it. - Cucubale porte-baies, Coulichon fr.

Fiore verde bianchiccio, frutto bacciforme nero.

\section{Gen. - $6^{0}$ จillene Lin.}

\section{A) Calice gonfio.}

- Petali bipartiti.

S. inflata Smitr. (Cucubalus Behen Re-Lin.). Volgare nei prati.

Per. Maggio, Settembre. (Fiori bianchi).

Bubbolini it. - Silène enflée, Carnillet fr. 
* S. alpina Thomas. - Cenisio a Ronche (Bouvier). Siti alpestri nella vallée etroite a Bardonnêche (Valino, Boll. del Club Alp. it., $\left.\mathrm{n}^{0} 35\right)$.

Per. Luglio, Agosto. (Fiori bianchi o violetti).

Silene alpina it. - Silène des alpes fr.

(Silene unifiora $\beta$ DC. Fl. Fr.).

* * Petali interi o quasi.

S. conoidea LIN. - Nei campi.

Silene a trombone it. - Silène conoide fr.

Ann. Giugno, Luglio. (Fiori rosei).

Sulla Brunetta (Negri).

B) Calice tubuloso.

* Lamina dei petali sprovrista di squame.

S. italica Pers. (Cucubalus italicus Re-Lin.). - Siti sassosi intorno Susa.

Per. Maggio, Giugno.

Silene delle selve it. - Silène d'Italie fr.

Petali bifidi bianchi con vene violacee al disotto.

S. otites Smith. (Cucubalus otites Re-LrN.). - 0 vunque nei siti sabbiosi lungo la Dora ed i torrenti.

Per. Giugno, Luglio. (Petali verdognoli).

Sesamoide grande it. - Silène à petites fleurs fr.

- Lamina dei petali provvista alla base di due squame.

a) Pelali smarginati.

S. armeria Lis. - Abbonda nei declivi sassosi di Condove, delle Blaccie e lungo la Dora.

Ann. Giugno, Agosto. (Petali rosei).

Silene a mazzetti it. - Silène armeria fr. 
b) Petali bilobati.

S. rupesiris Lin. - Raccolta da me nei siti montani tra Monpantero e Foresto.

Per. Luglio, Agosto.

Silene a orecchio di topo it. - Silene des rochers fr.

Fiori bianchi o roseo-sbiaditi.

Cenisio (Bouvier) - Colle della Portia - Sagra di S. Michele.

\section{S. acaulis Lis. - In tutti i pascoli alpini.}

B. Elongata BeLl. - Questa varietà di S. acaulis si trova nelle selve alpine sopra Monpantero e nei siti montani del Cenisio.

C. Excapa AlL. - Frequente nei pascoli alpini.

Per. Luglio, Agosto.

Ocimoide muscoso it. - Silène à courte tige fr.

Bellissima specie alpina a foglie di color verde gaio ed a fiori talvolta di color roseo vivissimo talvolta d' un roseopallido delicatissimo (nella Excapa). La S. acaulis cresce in fitti cespugli che i frequentatori delle alpi ammirano e ricordano sempre con piacere.

c) Petali 4-dentati.

* S. quadrifida Lis. - Cenisio. (Bonjean in Bertol.).

Per. Giugno, Agosto. (Fiori bianchi).

Silene dei fonti it. - Silène quadrifide fr.

* S. alpestris JACQ. - Alpi di Bussolino fra la Balmetta e il Colle dell' Orsiera.

Per Luglio, Agosto (Fiori bianchi).

Silene dentellata it.

Trovata la prima volta (1876), nella località accennata, dai giardinieri del R. Orto bot. di Torino, Chiuso e Defilippi. Pianta ritenuta prima come propria delle alpi Venete. 
d) Petali bifdi.

S. nutans Lin. - Frequente nei pascoli e presso le selve.

Per. Luglio, Agosto. (Fiori bianchicci).

Silene ciondola it. - S. penchée fr.

S. viridiflora Lin. - Intorno Susa.

Per. Maggio, Giugno. (Fiore bianco verdognolo).

Ocimastro verde it. $-\mathrm{S}$. à fl. vertes fr.

S. saxifraga Lin. - Luoghi aprici nelle rupi delle alpi di Mocchie.

Per. Giugno, Agosto. (Fiori bianchi).

Silene rupina it. - S. saxifraga fr.

S. vallesia Lrs. - Ovunque nei siti montuosi. Per. Giugno, Agosto.

Viscagine persichina it. - S. du Valais fr.

Cenisio alla Mergerie e tra le Ferriere e la Novalesa. (Bonjean in Bertol.).

Petali sopra roseo-pallidi - sotto venati di rosso.

3) Stili 5.

GeN. 10 - Lyehmis LIN.

- Dioiche.

L. diurna Sibthorp. (L. dioica Re a Lin.). - Prati alpini umidi.

Per. Giugno, Agosto. (Fiori porporini).

Ocimoide it. - Lychnide des bois fr.

Cenisio (Bonjean in Bertol.).

* L. vespertina Siвтновр. - Ovunque nelle siepi e nei margini dei campi. 
Bien. Giugno, Agosto. (Fiori bianchi).

Gittone bianco it. - Orie d' gat vern. - Lychnide dioique - Oeiliet de Dieu fr.

(Lychnis dioica $\beta$ ) Lin. Dalla località si può desumere che per Lychnis dioica il Re volle intendere la prima di queste due specie riportata sotto il nome di Lychnis sylvestris nella Fl. Tor. dello stesso autore.

\section{* Ermafrodite.}

(Fiori porporini, raramente bianchi).

a) Petali quadripartiti.

L. flos-cuculi Lin. - Ovunque nei prati irrigui.

Per. Maggio, Giugno.

Tenerone - Fior del cuculio it. - Fleur de coucon fr.

b) Petali bifidi.

L. alpina LiN. - Sommita del Cenisio.

Per. Luglio, Agosto.

Violino d'alpe it. - Lychnide des alpes fr.

Cenisio all'Eau blanche (Bonjean in Bertol.) (Negri).

L. flos-Jovis Lamk. (Agrostemma flos-Jovis RE-LIN.). - Cenisio alla posta, e nella discesa del piccolo Cenisio.

Per. Giugno, Luglio.

Fiore di Giove it. - Fleur de Jupiter fr.

c) Petali smarginati.

L. viscaria LrN. - Frequente nei pascoli.

Per. Maggio, Giugno.

Viscaria it. - Lychnide viscaire fr.

Cenisio (Bonjean in Bertol.). 


\section{Ordine XIV - Alsinee (Alsinea DC.).}

A) Numero delle valve della capsuta pari a quello degli stili.

\section{1) Stili 2 - Valve 2 .}

\section{GEN. $1^{\circ}$ -}

\section{B. tenuifolia Lis. - Nei campi presso Oulx.}

Ann. Maggio, Giugno. (Fiori bianchi, foglie filiformi, pet.4).

Stellina giuncolina it. - Buffonie à feuilles menues $\mathrm{fr}$.

Valle di Oulx (Re in Bertol.).

\section{2) Stili 3 - Walve 3 .}

\section{Gen. $2^{\circ}$ - Cherlerin Lin.}

C. sedoides Lrx. - Pascoli sassosi del Cenisio. Per. Luglio, Agnsto (Apetala).

Renaiola borracina it. - Cherleria faux sedum fi.

\section{Gen. $3^{\circ}$ - Isime Wahlenb.}

(Tutte le specie hanno fiori bianchi).

- Foglie lineari subulate senza nervi, o uninervate

A. laricifolia Wahlenb. (Arenaria striata Re-Lin.) - Nei siti sassosi montani di Chianoc, del Cenisio e delle alpi di Giaveno.

Per. Luglio, Agosto.

Renaiola pelosa it. - Alsine à feuilles de meleze fr. Musinè sotto Aren. laricifolia (Re, Fl. Tor.).

* Foglie angusto-lineari trinervate almeno allo stato secco.

A. villarsii Mert. e Kock. (Aren. austriaca Re-At.c.) - Alpi di Chianoc e del Cenisio. 
Per. Giugno, Agosto.

Renaiola savojarda it. - Alsine de Villar fr.

Nel testo Aren. austriaca Lin. dal Wild. spee.

* A. verna Bartl. - Cenisio (Balbis, Bonjean in Bertol.). (Negri) Assietta.

Per. Giugno, Agosto.

Renaiola primaticcia it. - Alsine du primtemps fr.

* B. Alpina Koch (Arenaria liniflora Jacq.). Cenisio (Bouvier). Fiorisce con la specie.

* A. recurva Wahlenb. - Cenisio (Bouvier).

Per. Luglio, Agosto.

Renaiola falcata it. - Alsine recurbée fr.

* A. rostrata Kock. - Sulla Brunetta: sul Cenisio (Negri).

Per. Giugno, Luglio:

A. Jacquinii Kock. (Aren. fasciculata RE-JAcQ.) e (Alsine mucronata Re-Balbis Misc. alt.). Pascoli sassosi montani sopra Foresto e sopra Chianoc andando alla Comba. Nasce pure a Monpantero ed alle Ferriere.

Ann. Giugno, Luglio.

Renaiola a capolini it. - Alsine de Jacquin fr.

Dintorni sassosi di Susa, Cenisio (Balbis, Re, Badaro, Bonjean in Bertol).

Nel testo Arenaria fasciculata, e Alsine mucronata Lin.

A tenuifolia Wahlenb. (A. tenuifolia Re-Lin.) - Volgarissima nelle rupi presso Susa e nelle mura delle vigne.

Ann. Maggio, Giugno.

Renaiola dei muri it. - Alsine à feuilles menues fr. 
A. lanceolata Mert. E Koch. (A. lanceolata Re-All.). - Cenisio verso la Novalesa.

Per Giugno, Agosto.

Renaiola sassefrica it. - Alsine lancéolée fr.

(Facchinia lanceolata Reichenb).

\section{Gen $4^{\circ}$ - Spergoularia Pers.}

S. rubra Pers. (Arenaria rubra Re-Lin.) - Luoghi sabbiosi presso Susa.

Ann. Maggio, Settembre. (Foglie stipulate - Fiori rosei). Spergola it. - Spergulaire rubre fr.

Cenisio presso le caserme (Bonjean in Bertol.). (Lepigonum rubrum Wahlenb).

3) Stili 1-5 - Valve

GeN. $5^{\circ}$ - Sagina LiN.

(Fiori bianchi - foglie senza stipule, congiunte, vaginate alla base. - Stili 4 - Valve 4.

S. procumbens LiN. - Nei siti ombrosi.

Ann. Maggio, Settembre.

Budellina dei fonti it. - Sagine couchée fr.

S. apetala Lrn. - Siti aridi presso le mura. Ann. Aprile, Miaggio. (Apetala o con petali minutissimi). Budellino dei muri it. - Sagine apetale fr.

* Stili 5 - Valve 5.

* S. saxatilis Wrms. - Cenisio (Bertol.). sotto (Spergula saginoides Lin.).

Per. Giugno, Luglio.

Spergola budellina it. - Spargoute fausse sagine fr. 
* S. glabra Koch. - Cenisio (Bouvier) (Spergula saginoides All.).

Per. Luglio, Agosto.

Spergula serpeggiante it. - Sagine glabre fr.

\section{Gen. $6^{\circ}$ - Spergula Lin.}

(Fiori bianchi, foglie libere alla base; stipulate).

S. arvensis Lin. - Frequente nei campi.

Ann. Giugno, Luglio.

Renaiola, spergola it. - Spergule des champs fr.

B) Valve o denti delle valve in mumero doppio degli stili.

1) Stili $\$$ - Ealve $\$$.

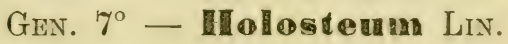

H. umbellatum Lis. - Volgare nei campi collinosi di S. Lazzaro presso Susa.

Ann. Aprile, Maggio. (Fiori bianchi in ombrella).

Centonchio a ombrella it. - Holostée en ombelle fr.

Gen. $8^{\circ}$ - remaria Lin.

(Fiori bianchi).

* Foglie ovato-accuminate o sub-rotonde.

A. serpyllifollia LiN. - Frequente nei siti sterili e caldi. Alle Blaccie presso Susa.

Ann. Maggio, Agosto.

Erba pondina it. - Sablines à feuilles de serpolet fr.

A. ciliata LrN. - Ovunque nei pascoli del Cenisio.

Per. Luglio, Agosto. (Foglie talvolta lanceolate).

Renaiofa sermollino it. - Sabline ciliée fr. 
A. biflora LrN. - Copiosa nel Cenisio a l'Eau Blanche, e nel piccolo Cenisio.

Per. Luglio, Agosto.

Renaiola quattrinella it. - Sabline à deux fleurs fr.

** Foglie lineari lesineformi aristate.

A. grandiflora AlL. - Alpi di Chianoc e del Cenisio.

Per. Luglio, Agosto.

Renaiola restata it. - Sabline à grandes fleurs fr.

A. saxatilis Lin. - Nella valle d'Oulx, secondo scrive il chiar. Allioni.

Specie assai dubbia come pertinente alla Flora. Arenaria saxatilis Lin. non è italiana. La pianta a cui accenna il Re è probabilmente una forma di Alsine verna Bartl. rapportata dal Bertol. ad Arenaria saxatilis All. non Lin. (Bertol., vol. IV; pag. 670-71).

\section{GEN. $9^{\circ}$ - Moellorimgia LIN.}

(Fiori bianchi).

M. trinervia Claint. (Arenaria trinervia RE-LiN.) Ovunque nei siti ombrosi.

Ann. Maggio, Giugno.

Renaiola nervosa it. - Moehringie à trois nervures fr.

* M. polygonoides MerT. e Koch. - Cenisio (Bonjean in Bertol.)

Per. Giugno, Luglio.

Renaiola uccellina it. - Moehringie fausse renouée fr.

M. muscosa Lin. - Tra i sassi nei siti montani ombrosi.

Per. Giugno, Luglio.

Centonchio minore it. - Moehringie mousse fr. 
Nella M. muscosa manca, come in varie Alsinee, una parte dei verticilli fiorali. Ha 4 sepali, 4 petali, 2 stili, 4 valve. Musinè - Fontana del trucchetto - Sagra di S. Michele.

\section{GEN: 10. - Gellaria LIN.}

(Fiori bianchi - Petali bipartiti o bifidi).

- Capsula a deiscenza profonda.

S. nemorum Lin. - Cenisio e in tutte le selve ombrose, montane ed alpine.

Per. Maggio, Luglio.

Centonchio montano it. - Stellaire des bois fr.

S. media Vill. (Alsine media Re-Lin.) A. media 0 morsus gallinae Off́. - Pavaronia vern. - Niente di più volgare negli orti.

Ann. Marzo, Ottobre.

Centonchio it. - Stellaire intermediaire, morgelinet fr.

La capsula coi semi è cibo graditissimo agli uccelli granivori.

Farmaco da lungo tempo disusato.

S. holostea Lin. - Trovata da Ignnazio Molineri sopra Giaveno andando al Colle della Rossa.

Per. Maggio, Giugno.

Stellaria garotanina it. - Stellaire holostée fr.

S. graminea LiN. - Nei pascoli umidetti lungro il Sangone.

Per. Giugno, Luglio.

Stellaria gramignola it. - Stellaire graminée fr.

S. uliginosa Murray. (S. hypericifolia Re-All.). Presso le fonti nei siti montani ed alpini.

Ann. Maggio, Giugno.

Stellaria palustre it. - Stellaire aquatique fr. 
* Deiscenza limitata all'orlo della capsula.

S. cerastioides Lin. (Cerastium refractum RE-ALL.) - Trovato da Ignazio Molineri nei siti elevati del Cenisio a l'Eau Blanche.

Per. Luglio, Agosto.

Stellaria orecchio di topo it. - Stellaire faux ceraiste fr. Eau Blanche nel Cenisio (Bonjean in Bertol.) (Cerastium tryginum Vill.).

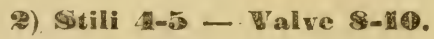

\section{GeN. 11. - Moenchia EHRH.}

(Fiori bianchi - Petali interi o quasi).

M. erecta Smith. (Sagina erecta Re-LIN.) - Trovata da Ignazio Molineri nei campi macilenti presso Caselette.

Ann. Aprile, Maggio.

Burinella it. - Céraiste dressé fr.

M. mantica B.nrtu. (Cerastium manticum Re-LiN.) Nei campi.

Ann. Maggio, Giugno.

Cerastio stellato it. - Céraiste trompeur fr.

* Gen. 12. - Censustium LIN.

(Fiori bianchi - Petali bifidi.).

- Petali uguali ai sepali o pitı brevi. .

G. glomeratum Thuilt. (Cerastium vulgaium RE-LIN.) - Ovunque nei siti macilenti.

Per. Aprile, Agosto.

Cencio molle it. - Céraiste commun fr. 
C. semidecandrum Lin. - Frequente nei pascoli. Ann. Aprile, Maggio.

Muschio di prato it. - Céraiste à 5 anthères fr.

G. triviale Link. (C. viscosum RE-LiN.) - Dovunque nei siti sabbiosi lungo la Dora.

Cerastio viscoso it. - Céraiste visqueux fr.

- Petali del doppio pit lunghi dei sepali.

\section{Alpinum Lin. - Cenisio.}

Per. Luglio, Agosto.

Orecchio di topo d'alpe it. - Céraiste des alpes fr.

Cespitoso - Foglie ovali o lanceolate - Pedicilli sfiorati patenti obliqui: brattee scariose all'apice.

\section{Iatifolium Lin. - Cenisio.}

Per. Luglio, Agosto.

Orecchio di topo d'alpe it. - Céraiste à larges feuilles fr. Cespitoso - Foglie più grandi che nel C. alpinum - Pedicilli sfiorati riflessi: brattee interamente erbacee.

C. arvense Lix. (C. lineare RE-Alt.) - C. sirictum Re-(Lin. ?) - Abbonda nelle rupi intorno Susa e specialmente alle Blaccie. Nel Cenisio e nei siti ombrosi alpini di Giaveno.

Per. Maggio, Luglio a seconda della località.

Ha le foglie lineari-lanceolate o lincari; e le brattee scariose. I Pedicilli sfiorati eretti.

Corastium strictum di Lin., e dei botanici non recenti, fra i quali il Re, che lo riportano sotto questo nome, è una pianta assai dubbia; anzi il Koch opina che Lin. l' abbia scambiata per Arenaria grandiflora All.

Le specie di Cerastium, come in generale le Alsinee, sono polimorfe a sommo grado, e divise e suddivise dagli autor'i in un'infinità di specie e di varietà; la loro sinonimia è per conseguenza intricatissima. 
A queste due supposte specie: Cerastium strictum e Cerastium lineare, che veramente non si possono considerare che come varietà del C. arvense, aggiungasi una terza, anch'essa varietà dell'arvense, Cerastium laricifolium Vill. Balbis, che il Re nella sua quarta appendice inedita alla Fl. Piemontese dice di aver trovato presso Susa a piedi del monte di San Lazzaro. (Colla, Herb. Ped.).

\section{\$) Stin \$ - Valve to profondamente dlentate.}

\section{Gen. 13. - Malachium Fries.}

(Fiori bianchi - Petali bipartiti).

M. aquaticum Fries. (C. aquaticum Re-Lin.). - Volgare nei siti umidi e pingui.

Per. Giugno, Agosto.

Budellina d'acqua it. - Malachie aquatique fr.

Ordine XV. - Linee (LineC DC).

Gen. - Limen Lin.

A) Sepali con ciglia glandulose.

- Petali gialli - Foglie sparse.

L. gallicum Lrs. - Copiosissimo nei pascoli aridi e sterili di Condove nella contrada detta La Rocca e nel Musinè.

Ann. Giugno, Luglio.

Lino spicato it. - Lin de France fr.

L. strictum Lrs. - Abbonda nei pascoli sterili sopra Foresto ed intorno alle vigne di Monpantero.

Ann. Maggio, Giugno.

Passerina bastarda it. - Lin roide fr.

Musinè, lato di mezzogiorno. 
" "Petali lilla-sbiaditi: foglie sparse.

\section{L. tenuifolium LIN. - Nei pascoli montani}

Ann. Giugno.

Lino montano it. - Lin à feuilles menues fr.

Musinè (Re, Fl. Tor.).

* * Petali bianchi : foglie opposte.

L. catharticum Lrs. e Off. - Ovunque nei prati macilenti e ombrosi. Amaricante, catartico.

Ann. Giugno.

Lino catartico it. - Lin purgatif fr.

Purgante, diuretico: disusato.

B) Sepali nudi - Fiori cerulei.

\section{L. alpinum Lis. - Nel Cenisio.}

Per. Giugno, Luglio.

Lino celeste it. - Lin des alpes fr.

Cenisio al Lago, alla Ramasse (Bucci, Malinverni in Parlat. Fl. it.) Assietta.

* L. usitatissimum LiN. - Il lino coltivato.

Ann. Luglio, Agosto.

Questa specie originaria dell' Asia settentrionale ed occidentale è senza dubbio una delle piante industriali più utili all'uomo, e fu da esso coltivata da tempi immemorabili. Basta notar questo, che sono di lino le tele nelle quali sono avvolte le antichissime mummie egiziane.

Oltre la fibra tessile il lino coltivato produce semi dai quali si estrae un olio conosciutissimo sotto il nome di olio di lino, adoperato in pittura e nella composizione dell' inchiostro tipografico. Sfarinati i semi del lino cotti ed applicati esternamente (cataplasmi di farina linosa) sono un rimedio emolliente e antispasmodico eccellente. 
Ordine XVI - Malvacee (Malvacece Juss.).

\section{Gen. $1^{0}$ - Malva LiN.}

- Peduncoli ascellari solitari: fiori rosei.

M. Alcea Lin. - Alcea vulgaris Off. - Piuttosto frequente ai margini delle vie e negli spineti. Mucilaginosa emolliente.

Per. Luglio, Agosto.

Alcea it. - Mauve alcée fr.

* y Fastigiata Koch. (M. Morenii Poll. Fl. Ver.) Caselette (De Filippi).

\footnotetext{
- Peduncoli ascellari fascicolati:

fiori rosei o bianchicci venati di rosso.
}

M. silvestris LIN. - M. vulgaris flore majore Off. Malva, o Riondella vern. - Intorno alle case nei siti incolti e pingui e nelle siepi degli orti. Virtù della precedente.

Bien. Giugno, Settembre. (Caliculo a foglioline oblunghe). Malva selvatica it. - Mauve sauvage fr.

M. rotundifolia LIN. - M. vulgaris flore minore 0ff. - Malva, o Riondella vern. - Frequente nei ruderi. Virtù delle precedenti.

Ann. Giugno, Settembre. (Caliculo a foglioline. lineari). Malva comune it. - Mauve à feuilles rondes fr.

\section{Gen. $2^{\circ}$ - Mlhaea LIN.}

(Fiori rosei o bianco-rosei).

* Foglie ovali a 3-5 lobi.

A. officinalis Lin. - A. Hibiscus - Bismalva o Mal- 
vaviscus Off. - Altea vern. - Lungo i fossi. Emolliente, mucilaginosa.

Ann. Giugno, Agosto.

Altea it. - Guimauve officinale $\mathrm{fr}$.

* Foglie, almeno le superiori, profondamente partite.

A. cannabina Lin. - Nelle siepi e nei spineti.

Per. Luglio, Agosto.

Alcea it. - Guimauve à feuilles de Chanvre fr.

A. hirsuia Lin. - Nei colli aprici.

Ann. Giugno, Agosto.

Cannabina ispida it. - Guimauve herissée fr.

Tanto le tre specie di Malva quanto l'Altea Officinale non hanno perduto il grido di buoni rimedi emollienti. Sono adoperate le foglie in cataplasmi, ed $i$ fiori e le radici, specialmente dell'Altea, in siroppi e infusioni.

\section{Ordine XVil. - Tiliacee (Tiliacere Juss.).}

\section{Gen. Unico - Tilia Lin.}

\section{T. ulmifolia Scop. (T. europaea RE-LiN.) - T. Off.}

- Lungo la via da Susa a Gravere. Fiori antispasmodici.

Tiglio selvaggio it. - Tiglieul, Teit, vern. - Tilleul à petites feuilles fr.

Albero elegante, capace di acquistare, coltivato, grandi proporzioni. Ha fiori fragranti, che si aprono in Giugno, i quali insieme con le brattee infusi nell' acqua bollente spiegano un'azione diaforetica, e la moderna terapia li tiene in buon conto. - Il suo legno è bianco e facile a lavorarsi. 
Ordine XVIII. - Ipericinee (Hypericinece DC.).

\section{GeN. - Hyperieum LiN.}

(Fiori gialli).

A) Pianta irsuta - Sepali interi, glandolosi nel margine.

H. hirsułum Lin. - Nei monti presso le selve. Per. Luglio, Agosto.

Iperico peloso it. - Millepertuis herissé fr.

B) Piante glabre - Sepali interi non glandulosi nel margine. - Caule pit o meno ancipite.

H. humifusum Lin. - Abbonda nei monti di Giaveno.

Per. Luglio, Agosto. (Cauli filiformi prostrati). Iperico filiforme it. -- Millepertuis couchè fr.

H. perforatum Lis. - H. o Perforata Off. - Frequente nei pascoli. Le cime fiorite sono vulnerarie.

Per. Luglio, Agosto.

Iperico, Perforata it. - Ipericon vern. - Millepertuis fr.

Cauli eretti: le foglie di questa specie come delle due seguenti hanno sembianza di essere perforate in moltissimi punti della loro superficie. Questa pianta fu reputata un giorno vulneraria, astringente, diuretica e vermifuga:ilsuo impiego farmaceutico non è però del tutto abbandonato ai giorni nostri.

I fori delle foglie non sono in realta che vescicole trasparenti contenenti un'essenza di odore speciale penetrante, a molte persone nauseoso.

* Caule quadrangolare.

H. tetrapterum PeRs. (H. quadrangulare RE-Willd.) - Nei siti umidi e lungo i fossati. 
Per. Giugno, Luglio.

Iperico quadrangolare it. - Millepertuis tétragone - Ascyron fr.

Nel testo H. quadrangulare Lin. dal Willd. spec.

H. quadrangulum Lin. (H. maculatum Re-CrANTZ) Abbonda nel Cenisio e nelle alpi di Chianoc.

Per. Luglio, Agosto.

Iperico reticolato it. - Millepertuis douteux fr.

Al $5^{\circ}$ ricovero, al lago del Cenisio: (Parlat., Fl. It.).

\section{H. crispum Lin.-Mant. - Cenisio.}

\section{Per. Maggio, Giugno.}

Iperico ondulato it. - Millepertuis crepu fr.

Specie non appartenente alla Flora nostra; H. crispum è proprio del mezzogiorno italiano e vive specialmente in Sicilia (Bertol., Parlat.). Allioni (Ped.) scrisse che si trova sul Cenisio; e del Cenisio, forse su la fede dell Allioni, lo riportarono parecchi autori, fra i quali Re, Decandolle (Fl. Fr.) e più recentemente L. Reichenbach (Fl. Ger. Exc.).

C) Piante glabre - Sepali dentati o frangiati, glandolosi.

* Foglie verticillate a $4-5$.

H. Coris Lis. - Frequentissimo nei siti aprici intorno Susa.

Per. Maggio, Settembre. (Petali spesso striati in rosso). Cori it. - Millepertuis verticillè fr.

* Foglie opposte.

H. montanum Lin. - Cenisio, Comba di Chianoc.

Per. Giugno, A gosto.

Iperico montano it. - Millepertuis de montagne fr. 


\section{H. Richerii Vill. (H. barbatum RE-ALL.) - Il chiar.} Allioni affermò che trovasi nel Cenisio.

Per. Giugno, Luglio.

Iperico frangiato it. - Millepertuis de Richer, Milleper. frangé fr.

Cenisio (Bertero, Barbieri in Parlat. Fl. it.) (Bouvier).

\section{Ordine XIX. - Acerinee (Acerine DC).}

\section{Gen. Unico - Acer Lin.}

* Filamenti irsuti alla base: fiori in racemi pendenti.

\section{A. pseudoplatanus LiN. - Siti montani ed al-} pestri. Frequente nella contrada di Fossimagna.

Fiorisce in Maggio, Giugno.

Acero fico-sicomoro it. - Plaie vern. - Érable faux platane, Sycomore fr.

Musinè (Re, Fl. Tor.).

Albero bello, grandioso, capace di elevarsi oltre i 25 metri. Il suo legno compatto e nel medesimo tempo facile a lavorarsi è adoperato per costruire arnesi domestici, forme per fare il formaggio (Cuppe vern.), strumenti musicali, ecc. Il suo tronco inciso in primavera dà un sugo dolce che, bollito, si rapprende in zucsaro.

* Filamenti glabri.

a) Racemi eretti corimbiformi.

A. platanoides Lin. - Presso la fabbrica del marmo.

Fiorisce in Maggio, Giugno.

Albero riccio it. - Érable de Norvège, Érable plane fr.

Albero di portamento bellissimo, capace anche esso di portarsi a rilevante altezza (18-25 metri); utilissimo anch'esso per lavoro. 
A. campestre LiN. - Obi vern. - Volgare dovunque.

Fiorisce in Maggio.

Oppio loppio it. - Érable commun fr.

Alberetto capace però di acquistare con la coltura e col tempo grandi dimensioni. 亡̀ adoperato, in Toscana specialmente, a sostegno delle viti.

b) Racemi corimbiformi pendenti.

A. opulifolium VILL. - Plaie vern. - Siti montani e caldi sopra Chianoc andando alla Comba. Frequente nei monti di Bussolino, al Tempio della Sagra di S. Michele, ed anche alla Losa nei monti di Susa.

Fiorisce in Maggio.

Dintorni di Susa (Parlat., Fl. It.).

Albero anch'esso bello, ma di minori dimensioni dell' A. pseudoplatanus, denominato in dialetto esso pure Plaje, eccellente, come quello, per lavori svariati.

* Ordine XX. - Ippocastanee (Hippocastanea DC.).

\section{Gen. Unico - Aesculus Lin.}

\section{A. hippocastanum LiN.}

Fiorisce in Maggio.

Castagno d'India it. - Marronier d'Inde fr.

Quest'albero magnifico, originario dell'Asia ed introdotto in Europa da oltre due secoli, sembra fatto a posta per decorare parchi e ombreggiare i pubblici viali. Resiste a fredde latitudini ed è ammirabile per la precocità delle sue foglie. Dalla sua corteccia fu estratta una sostanza (l' Esculina) amara e tonica stimata da taluni terapisti come buon succedaneo dei sali di China. 
Ordine XXI - Ampelidacee (Ampelidece Kunth.).

\section{GEN. $1^{0}$ - Vitis LIN.}

\section{V. vinifera LrN. - Nelle selve e negli spineti.}

Fiorisce in Giugno.

Vite it. - Vigne fr.

Arbusto sarmentoso coltivato a tutti noto e capace di assumere le dimensioni più disparate ed ubbidiro, quasi diremmo, a tutti gli umani capricci. Le varietà di viti sono innumerevoli.

Questa pianta si crede sia originaria dell' Asia, trasportata e naturalizzata in Europa da tempi antichissimi.

* Gex. $2^{\circ}$ - Ampellopsis Michaux.

\section{* A. hederacea Мich.}

Arbusto scandente Luglio, Agosto.

Vite vergine it. - Vigne vierge $\mathrm{fl}$.

Coltivata per tappezzare le mura dei giardini e delle case di campagna.

Ordine XXII. - Geraniacee (Geraniacece DC.).

GeN. $1^{*}$ - Germminm LIN.

(Fiori purpurei, rosei, o violacei).

A) Rizoma più o meno orizzontale.

- Valve con rughe trasversali.

* G. macrorhizum Lrs. - Cenisio (All. Ped.).

Per Giugno, Agosto.

Geranio crestato it. -- Geranium à grosses racines fr.

Re non riporta questa specie e forse a ragione. Il Pallatore (Fl. It., rol. V, pag. 162-63) dice: "Allioni scrive che si dice nascere sul Cenisio, peró non vi è stato trovato, per quanto io mi sappia, da alcun botanico moderno ”. 
G. phaeum Lin. (Ger. fuscum Re-BeLl.) - Alla Comba sopra Chianoc.

Per. Maggio, Giugno.

Geranio stellato it. - Geranium livide fr.

Cenisio (Bonjean in Bertol.) (Huguenin in Parlat. Fl. it.).

* Valve liscie, glabre o pelose.

G. nodosum Lin. - In tutte le selve.

Per. Giugno, Luglio.

Geranio nodoso it. - Geranium nodeux fr.

G. sylvaticum LrN. - Volgare in tutte le alpi Presso Susa, nel sito detto Le Gorgrie.

Per. Giugno, Agosto.

Geranio silvano it. - Geranium des bois fr.

Musinè (Re, Fl. Torin.). ('enisio (Bonjean in Bertol.).

G. palustre LiN. - Trovata dal chiar. Allioni presso il lago del Cenisio.

Per. Luglio, Agosto.

Geranio palustre it. - Geranium des marais fr.

Pianta molto dubbia come pertinente alla nostra Flora. Allioni forse ritenne per Geran. palustre di Linneo qualche forma del Geran. sylvaticum, che vegeta sul Cenisio. Geran. palustre Lin. è proprio delle alpi Bergamasche, Bresciane e Friulane. (Parlat., Fl. It.). Il Colla (Herb. Pedem., vol. 1, pag. 506), scrive ch'egli non lo rinvenne nel sito indicato da Allioni.

G. aconitifolium WILLD. - Trovato da Ignazio Molineri nelle Cave di gesso al di là della Posta. nel Cenisio, come si può rilevare dalla miscell. Bot. del chiar. prof. Balbis.

Per. Giugno, Agosto.

Geranio lupaio it. - Geran. à feuilles d'aconit fr. 
Cenisio alla Ramasse e alla Margerie (Bonjean, in Bertol.) (Huguenin in Parlat., Fl. it.).

G. sanguineum LiN. - Geran. sanguineum ovvero Haematodes sanguinaria Off. - Ovunque nei pascoli aridi e sassosi. Farmaco disusato.

Per. Giugno. Agosto.

Geranio sanguigno it. - Geran. sanguine fr.

B) Radice fusiforme discendente robusta, perenne, Vaive liscie.

G. pyrenaicum Lin.-Mant. - Allioni scrive che fu raccolto intorno Susa.

Per. Aprile, Maggio.

Geranio malvino it. - Geranium des Pyrenées fr.

C) Radice fusiforme discendente, gracile, annua.

- Valve liscie.

G. columbinum LrN. - Ai margini delle vie, nei siti ombrosi.

Ann. Giugno, Luglio.

Piede colombino it. - Geranium colombin fr.

G. dissectum Lin. - Vie e siepi.

Ann. Maggio, Luglio.

Geranio sbrandellato it. - Geran dissequé fr.

G. rotundifolium Lis. - Ovunque nei pascoli. Ann. Giugno, Settembre.

Malvaccino it. - Geran. à feuilles rondes fr. * Valve rugose.

G. robertianum LrN. - Frequentissimo nelle mura ai siti ombrosi. 
Ann. Aprile, Giugno.

Cicuta rossa it. - Geran. herbe à Robert fr.

\section{Geñ. $2^{0}$ - Erodiuam L'Herit.}

- Filamenti glabri.

E. cicutarium L'Herit. (Geranium cicutarium Re-LiN.) - Ovunque, pascoli e vie. Ann. Aprile, Ottobre.

Erba cicutaria it. - Erodium à feuilles de Cigué fr.

E. malacoides Willd. (Geranium malacoides RE-LiN.) - Presso le vigne di Monpantero. Ann. Aprile, Maggio.

Altea bastarda it. - Erodium fausse mauve fr.

- Filamenti cigliati.

E. ciconium Willd. (Geranium ciconium Re-LiN.) Comune nei luoghi incolti presso le vigne. Ann. Maggio, Giugno.

Becco di grue it. - Erodium bec de Cicogne fr.

Ordine XXIII. - Balsamrnee (Balsaminea A. Rich.). Gen. UnICO - Impatiens Lin.

1. Noli tangere LIN. - Siti ombrosi umidetti. Ann. Luglio, Agosto.

Erba impaziente it. - Herbe n'y teuchez pas, Merveille, Herbe de Sainte Cathérine fr.

Le valve della capsula sono elastiche e si aprono appena si toccano, donde il nome. 


\section{Ordine XXIV - Ossalidacee (Oxalidacece DC.).}

\section{Gen. Unico - Oxalis Lin.}

* Acaule: Fiori bianco-rosei venati di rosso.

\section{0. acetosella Lrn. - Acetosella Alleluja e Lujula Off. - Copiosa nei prati ombrosi e nelle selve.} Acida, refrigerante.

Per. Aprile, Maggio.

Alleluja it. - Erba dël Coucou, vern. - Oxalide, Oseille, Surelle, Pain de Coucou fr.

Le foglie deli' $O$. acetosella hanno sapore acidulo piuttosto piacevole. Contengono dell'ossalato di potassio e riescono diuretiche.

\section{* Caulescente: Fiori gialli.}

0. stricta Lin. - Nei siti sassosi sterili di Condove. Virtù della precedente.

Ann. Giugno, Ottobre.

Lujola maggiore it. - Erba givola dël Coucou vern. Oxalide droite fr.

Ordine XXV - Zigofillee (Zygophyllece R. Bronn.).

\section{Gen. Unico - Triboulus Lin.}

T. terrestris Lin. - Lungo la via presso San Giuliano e presso Durbiano andando a Matthie, e copiosamente ad Oriente della Chiesetta della Madonna della Coà.

Ann. Giugno, Settembre.

Tribolo terrestre it. - Tribule terrestre fr. 
Ordine XXVI - Rutacee (Rutace Juss). Gen. $1^{0}$ - Ruta LiN.

\section{R. graveolens Lin. - Nelle rupi presso le vigne di Monpantero.}

Per. Giugno, Luglio.

Ruta it. - Rue fétide fr.

\section{Gen. $2^{\circ}$ - Dietammus LIN.}

\section{D. albus Lin. - D. albus o Fraxinella 0ff. - Nei} colli aprici presso Susa. Antispamodico, vermifugo.

Per. Maggio, Giugno.

Frassinella it. - Fraxinelle d'Europe fr.

Questa bella pianta, degna dei griardini, ha il fiore grande roseo o bianco venato di rosso, e le foglie imparipennate, eccetto le inferiori che sono semplici. - I peli a base glandulosa, dei quali è vestito il racemo della Frassinella, presentano un curioso e singolare fenomeno. Se ad essi, specialmente di sera, e massime quando la fioritura tocea il suo apogeo, si accosta la fiamma d'una candela, l'atmosfera che circonda la pianta si accende a guisa di un piccolo fuoco d'artifizio. Taluni credono che l'olio essenziale delle glandule cvapori e si infiammi, e taluni altri, forse meglio, che l'olio stesso sia bruciato nelle glandule e l'incendio propaghisi rapidamente dall'un all'altro di questi serbatoi.

Come farmaco la Frassinella è pressochè disusata. 


\section{DIVISIONE SECONDA.}

\section{Caliciflore - (Caliciflores)}

Ordine XXVII -- Celastrinee (Celastrinea R. Bronn).

. Gen. - Evomymus Lin.

E. europaeus Lin. - Evon Off. - Barëtte d' preive vern. - Frequente nelle siepi e nelle selve. Velenoso.

Arbusto. Maggio, Giugno.

Evonimo, Fusaggine it. - Fusain d'Europe, Bonnet des pretres fr.

Le foglie ed i semi di Evonimo furono amministrati un giorno come emetici e purgativi, oggi sono rimedi ristretti in qualche paese al ceto campagnuolo.

L'Evonimo ha odore ributtante ed è instintivamente rifiutato dalle bestie.

Ordine XXVIII. - Terebintee (Terebinthacea Juss.).

\section{Gen. - Bh hus Lin.}

\section{R. cotinus LiN. - Cotinus o Coccygria Off. - Nelle} selve sopra Foresto. Astringente.

Arbusto, fiorisce Maggio, Giugno - Fruttifica, Luglio, Agosto.

Cotino scofano it. - Sumac des teinturiers, Fustet, Arbre à perruque fr.

Arbusto elegante per la sua inflorescenza, nella quale i pe- 
dicilli di moltissimi fiori sterili si allungano dopo la fruttificazione dei fertili, e si coprono di lunghi peli rossicci e figurano una bella chioma. Il legno di questo arbusto dà un colore giallo, che serve a tingere le lane e le sue foglie sono un ottimo succedaneo del Rhus coriaria adoperate per la concia delle pelli. Disusato come rimedio.

\section{Ordine XXIX. - Rhaunacee (Rhamnec R. Bronn.).}

\section{GEN. - Rhammus LIN.}
A) Stami 4 - Stilo bi-trifido.

* Foglie opposte o quasi -- Rami spinosi.

\section{R. cathartica Lin. - R. catharticus - R. solutivus 0} Cervi spina Off. - Nelle selve, alle Blaccie presso Susa. Bacche diuretiche, catartiche.

Arbusto, fior. Maggio, Giugno. - frutt. Settembre.

Spin cervino it. - Nerprun purgatif fr.

Le bacche dello Spin cervino contengono una sostanza amara (catartina), purgante energico, il quale però è quasi caduto in disuso nella farmacia umana, e rimasta in uso nella veterinaria.

Dalle bacche stesse mature cola un liquido rosso-violetto, il quale, reso verde dall'allumina o dalla calce, costituisce il verde di vescica dei pittori.

* Foglie alterne - Rami inermi.

R. alpina LrN. - Nelle selve di Fossimagna. Arbusto, fior. Maggio, Giugno - frutt. Agosto, Settemb. Ramno alpino it. - Nerprun des alpes fr.

R. pumila Lis. - Rupi del Cenisio alle Ferriere e sopra Cesana.

Frutice Maggio, Giugno.

Ramno spaccasassi it. - Nerprun nain fr.

Frutice tortuoso, prostrato, disteso sulle rupi; foglie ovatoerenate. 
B) Stami 5 - Stilo indiviso.

Foglie sparse interissime.

R. Frangula LiN. - Frangula Off. - Comune in tutte le selve.

L'alburno è catartico e i semi sono diuretici.

Arbusto, fior. Maggio, Giugno - frutt. Luglio, Agosto.

Frangola, Alno nero it. - Bourdaine, Bois noire fr.

Ha le stesse proprietà catartiche del Rh. catartica ed è adoperata, specialmente in Francia, dalle classi di campagna.

\section{Ordine XXX. - Papiglionacee}

(Papitionacece Lin. or'd. nat.).

Sotto Ordine $1^{\circ}$ - Stani Monadelfi o Diadelfi.

6́ezione ${ }^{2}$ - Legume uniloculare o pira o meno longiudinalmente biloculare (Gen. EPhaca Dxytropis Actragalus) per introflessione di una delle suture. Cotiledoni, nella germogliazione, fogliacei epigei (fuori terra).
A) Stami Monadelf.
i) Foglie semplici.

Gen. $1^{0}$ - Gemista Lin.

(Fiori gialli).

G. tinctoria LiN. - G. tinctoria o Flos tinctorium 0ff. - Genestra vern. - Comune nei prati macilenti e nei pascoli. Tinge in giallo, insipida $\left({ }^{*}\right)$.

Per. Giugno, Agosto. (Inerme, legume glabro).

Baccellina, Genestrella it. - Giansip, Brouttairola d'le tinture vern. - Genêts des teinturiers, Genestrelle fr.

(*) Crediamo che la parola insipida del testo sia un errore materiale. 
Vantata un giorno come farmaco diuretico insieme alle altre specie. La radice contiene una materia colorante gialla atta a tingere tele e lane.

* G. ovata WALDST e Kit. - Tra Alpignano, Rivoli e Caselette.

Suffrut. Giugno, Settembre. (Inerme, legume irsuto).

Ginestra pelosa it. - Gênets ì feuilles ovales fr.

$\beta$ humilior Bertol. (Genista mantica Pollin.) Musinè. (Re, Fl. Tor.).

Questa specie è considerata da parecchi botanici come varietà della precedente.

\section{G. germanica LIN. - G. tinctoria o germanica $\mathbf{0} f f$.} - Frequente nelle selve dei monti poco elevati. Suffrut. Maggio, Giugno. (Spinosa legume irsuto). Ginestra spinosa it. - Gênets d'Allemagne fr.

\section{æ) Foglie trifogliate.}

\section{Gen. $2^{\circ}$ - Cytisus LiN.}

(Fiori gialli).

- Tubo del calice breve, munito o non di una piceola brattea alla base.

a) Racemi laterali pendenti.

C. Laburnum Lrs. - Amborn vern. - Frequente nelle selve montane ed alpine. Legno sospetto.

Arbusto. Maggio, Giugno.

Laburno: Maggio ciondulo it. - Cytise aubour, Cytise ì grappes, C. faux Ebénier fr.

* C. alpinus MrLu. - Cenisio (Balbis Elench. Stirp. rec.). Musinè (Balbis in Re, Fl. Tor.).

Arbusto. Giugno, Luglio.

Avorniello d'alpe it. - Cytise des alpes fr.

I fiori e le foglie di queste due specie ingerite sviluppano nelle bestie dolori gastrici. 
b) Racemi terminali eretti.

C. nigricans LiN. - Presso le selve.

Frutice Giugno, Luglio.

Maggio it. - Cytise noircissant fr.

** Tubo del calice breve, munito di 3 brattee alla base: racemi eretti terminali.

C. sessilifolius Lin. - Nei pascoli sterili. Frutice Maggio, Giugno. Majella it. - Cytise à feuilles sessiles fr. *** Tubo del calice lungo.

a) Fiori terminali numerosi fasciculato-ombrellati.

* C. capitatus JACQ. - Novalesa, e dintorni di Susa (Bonjean in Bertol.).

Frutice Maggio, Giugno.

Citiso ombrellato it. - Cytise en tête fr.

b) Fiori laterali fascicolati a $2-4$.

C. hirsutus Lin. - Ovunque ai margini delle selve.

Frutice Maggio, Giugno.

Citiso sanguigno it. - Cytise herissé fr.

Gen. $3^{\circ}$ - Dmomis Lin.

1) Legumi eretti ovati.

- Fiori rosei, molto raramente bianchi.

0. antiquorum Lin. - Nei siti sassosi sulle sponde della Cenisia presso Susa.

Per. Giugno, Settembre.

Anonide it. - Bugrane épineuse fr.

(0 spinosa $y$. glabra DC. Prod.).

Alla Ferriera (Bonjean in Bertol.). 
0. spinosa $\beta$ Lin. ( 0 . arvensis Re-Alt.) - Anonis, Ononis o Resta boves Off. - Resta beu vern. Ovunque nei pascoli sassosi. Sub-acre, diuretica.

Per. Giugno, Settembre.

Anonide arresta bue it. - Bugrane épineuse fr.

Nel testo 0 . arvensis Lin. dall'Allioni.

La radice dell'Anonide, rimedio diuretico, assai vantato un giorno nella calcolosi, nelle arene renali e nelle idropisie dipendenti da cause removibili, non è del tutto fuori uso nella farmacia moderna.

* Fiori gialli.

O. Columnae ALl. - Nei siti elevati di Monpantero.

Ann. Maggio, Giugno.

Bulimacola montana it. - Bugrane de Columna fr.

0. minutissima Lin. -- Frequentissima nelle rupi intorno Sasa.

Per. Maggio, Luglio.

Bulimacola barbuta it. - Bugrane naine fr.

2) Legumi pendenti lineari turgidi.

* Fiori gialli.

0. natrix LiN. - Nei siti sabbiosi specialmente presso ai torrenti.

Per. Giugno, Settembre.

Erba bacaia it. - Bugrane natrix fr.

* Fiori rosei.

0. Cherleri Re-Alt. - Abbonda nei pascoli sopra Foresto.

Ann. Maggio, Giugno.

Bulimacola pelosa it. - Bugrane renversée fr. 
(0. reclinata LiN. $\beta$ minor Moris FI. Sard.).

Nel testo O. Cherleri Lin. dal sinonimo di Allioni (Vedi l'osservazione del Moris Fl. Sard., vol. 1, pag. 422, e Koch Syn., ed. $3^{\text {a }}$ pag. 137).

0. rotundifolia LrN. - Abbonda nelle rupi dietro la Brunetta verso Monpantero e nel Cenisio sopra Les Ferrières.

Per. Maggio, Giugno.

Cece selvatico it. - Bugrane à feuilles rondes fr.

0. Cenisia Liv. - Nel Cenisio ma rara. Vegeta pure nella valle di Bardonêche.

Per. Luglio.

Bulimacola gambata it. - Bugrane du mont-Cenis fr.

Cenisio (Balbis e Bonjean in Bertol.).

3) Foglie imparipennate.

GEN. 4. - Anthyllis Lin.

- Fiori gialli.

A. vulneraria Lin. - Vulneraria Off. - Frequente nei prati macilenti e nei pascoli.

Per. Maggio, Giugno.

Vulneraria it. - Anthyllide vulnéraire fr.

Farmaco disusato.

* $\beta$ Bertol. brevemente pubescente, apice della carena rosso. Cenisio (Bonjean in Bertol.).

${ }^{*} \varepsilon$ Bertol. nana, irsutissima, carena come in $\beta$ (Astragalus vulnerarioides All.). Cenisio all' Eau Blanche (Bonjean in Bertol.). Cenisio a Ronche (Bouvier).

* y Bertol. ali e carena sanguigno-purpuree. (Anthyllis Dillenii Schult). Cenisio (Bonjean in Bertol.). A Ronche (Bouvier). 
* Fiori porporini.

\section{A. montana Lin. - Nei monti di Cesana.}

Per. Luglio.

Vulneraria montana it. - Anthyllide des montagnes fr.

B) Stami diadelfi.

1) 虽 oglie trifogliate.

\section{Gen. 5. - Medicago Lin.}

(Fiori gialli eccetto nella M. sativa).

- Legume inerme.

M. sativa Lin. - M. sativa Off. - Volgare nei prati e nei pascoli.

Per. Giugno, Luglio. (Fiori violetti).

Erba medica, Cedrangola it. - Luzerne fr.

Questa leguminosa meritò il nome di regina dei prati, tanto per l'abbondanza del suo prodotto quanto pel suo valore nutritivo.

La medica da tempi immemorabili fu introdotta dall'Asia in Europa. Abbonda di calce e di potassa e sviluppa radici lunghissime. Ama percio il terreno calcare argilloso profondo.

M. falcata Lin. - M. sylvestris Off. - Nasce nei luoghi stessi.

Per. Giugno, Agosto.

Erba medica a fior giallo it. - Luzerne en faucille fr. Queste due specie di medica son disusate come rimedii.

M. Iupulina Lrs. - Comune nei prati e nei pascoli. Ann. Maggio, Settembre.

Trifoglino selvatico it. - Luzerne lupuline, Mignonette, Minette dorée fr. 
M. orbicularis All. - Campi e vignne ed anche nei pascoli sterili di Condove nella contrada detta la Roccea.

Ann. Maggio, Giugno.

Fontello it. - Luzerne orbiculaire fr.

Nel testo M. orbicularis Lin. dall'All. e dal Willd. spec.

* Legume spinoso.

M. minima Lamk. (M. hirsura RE-ALL.) - Nei pascoli. Ann. Maggio, Giugno:

Trifoglino lappoloso it, - Luzerne naine fr.

Nel testo M. hirsuta Lin. dall'Allioni.

Tutte le specie di medica a fiori gialli, quantunque di minore conto della sativa, sono mangiate con piacere dal bestiame.

\section{Gen. $6^{\circ}$ - Trigomella LiN.}

T. Monspeliaca Lin. - Nelle vigne di Susa, di Monpantero, di Foresto e di S. Giorgio.

Ann. Giugno, Luglio. (Fiori gialli piccoli in ombrella).

Sertola stellata it. - Trigonelle de Montpellier fr.

\section{Gen. $7^{\circ}$ - Merillotms TounnF.}

(Fiori gialli raramente bianchi).

M. officinalis Lamk. (Trifolium melilotus officinalis RE o. LIN.). - Nei siti incolti presso le acque. Emolliente.

Bienn. Giugno.

Meliloto gigantesco it. - Mélilote officinal fr. 
M. italica PERs. (Trifolium melilotus italica Re-LrN.).

- M. italica Off. - Mi occorse nelle vigne di Monpantero nel sito detto di S. Eusebio.

Bienn. Aprile, Maggio.

Coronella it. - Mélilote d'Italie fr.

Il meliloto officinale contiene un olio etereo (cumina) e non è rifiutato dalla terapia moderna come farmaco interno eccitante, carminativo, ed esterno, in forma di cataplasmi emollienti, risolventi e d'iniezioni eccitanti nel puerperio. Le bestie lo mangiano volentieri.

* M. parviflora Desf. (?) — Presso Susa. (Re, App. $4^{\mathrm{a}}$ ad Fl. Ped. ined. in Colla Herb. Pedem. vol 2., pag. 107).

Ann. Giugno, Luglio.

Tribolo granelloso it. - Mèlilote à petites fleurs fr.

\section{GEN. $8^{\circ}$ - Trifolium Lin.}

1) Fiori sessili disposti a spica sub-rotonda o oblunga sprovvisti di piccole brattee.

* Fiori porporini.

T. medium Lin. (Trifolium flexuosum Re-JACQ.). Siti argillosi ai margini delle vie.

Per: Giugno, Agosto.

Trifoglio serpentino it. - Trèfle intermediaire fr. Ottimo per pascolo.

T. rubens LIN. - Intorno alle selve e nei prati montani.

Per. Giugno, Luglio.

Trifolio rosso it. - Trèfle rouge fr.

Buono per pascolo. 
T. alpestre LiN. - Ovunque nei pascoli montani.

Per. Giugno, Luglio.

Trifoglio alpestre it. - Trêfle alpestre fr.

Eccellente per pascolo.

T. pratense LIN. - Trafeui vern. - Ovunque nei prati.

Per. Maggio, Settem bre.

Trifoglio dei prati it. - Trèfle des près fr.

Ottimo per pascolo.

T. angustifolium Lin. - Pascoli di Condove al sito detto la Roccea.

Ann. Giugno, Luglio.

Coda di volpe it. - Trèfle à feuilles étroites $\mathrm{fr}$.

* Fiori bianchi o bianco-rosei.

T. arvense Lin. - Volgare nei campi.

Ann. Luglio, Settembre.

Erba lepre it. - Trèfle des champs fr.

T. scabrum Lin. - Pascoli aridi e sterili di Foresto.

Ann. Maggio, Luglio.

Trifoglio dei muri it. - Trèfle scabre fr.

T. striatum LiN. - Trovato da Ignazio Molineri nei pascoli a Caselette a piedi del Musinè.

Ann. Maggio, Luglio.

Trifoglio volpino it. - Trèfle strié fr.

* " Fiori giallastri.

T. ochroleucum Lin. - Nei pascoli intorno alle vigne di Monpantero. 
Per. Giugno, Luglio.

Trifoglio giallognolo it. - Tréfle jaunatre fr.

2) Fiori disposti a spica subrotonda o in capitolo, quasi sessili nel $\mathrm{Tr}$ fragiferum, pedicillati piú o meno lungamente nelle altre specie, provvis $t^{i}$ di piccole bratteo.

* Fiori rosei, raramente bianchi.

T. fragiferum LiN. - Presso le vie, nei pascoli umidetti.

Per. Giugno, Settembre.

Trifoglio fragolino pagolino it. - Trèfle fraisier fr.

Eccellente per pascolo.

T. alpinum LiN. - Frequente nei prati e nei pascoli alpini.

Per. Giugno, Luglio.

Trifoglio alpino it. - Trèfle des hautes alpes fr.

(Acaule a fiori grandi speciosi qualche volta bianchi). $\dot{\mathrm{E}}$ mangiato con avidità dal bestiame.

* Fiori bianchi, bianco-rosei o bianco-gialli.

T. repens LirN. - Nei pascoli pingui e nei prati. Per. Maggio, Settembre.

Trifoglio bianco, ladino it. - Trèfle rampant fr.

Ottimo per pascolo.

T. montanum Lis. - Nei prati montani ed alpestri.

Per. Maggio, Luglio.

Trifoglio montano it. - Trèfle des montagnes fr.

Buono per pascolo.

* T. caespitosum Reyn. - Cenisio (Bonjean in Bertol.). Cenisio presso il lago (Negri). 
Per. Luglio, Agosto. (Fiori bianco-giallicci). Trifoglio rupino it. - Trèfle gazonaut fr. (Trifolium Thalii, Vill.).

* T. pallescens Schreb. - Presso il lago del Cenisio (Negri).

Per. Luglio, Agosto.

Trifoglio pagliarino it. - Trèfle palissant fr.

T. hybridum LrN. - Nei campi.

Per. Maggio, Settembre.

Trifoglio fistoloso it. - Trèfle hybride fr.

Buono per pascolo.

- Fiori prima gialli, poscia, invecchiando, pit o meno bruni, bianchicci nel T. filiforme.

T. agrarium LiN. - Abbonda nei prati.

Ann. Maggio, Giugno.

Trifoglio luppolino it. - Trèfle des campagnes fr.

T. procumbens LiN. - Prati e pascoli macilenti. Ann. Maggio, Settembre. Pratolino it. - Trèfle etalée fr.

T. filiforme Lin. - Nei prati.

Ann. Maggio, Giugno.

Trifoglio capillare it. - Trèfle filiforme fr.

T. spadiceum Lrs. - Nei prati e nei pascoli del Cenisio.

Per. Giugno, Agosto.

Trifoglio lucido it. - Trèfle brun fr.

* T. badium Schreb. - Altipiano del Cenisio. (Negri). Cenisio (Bonjean e Balbis in Bertol.). 
Per. Giugno, Luglio.

Trifoglio capolone it. - Trèfle brunissant fr.

Notammo i trifogli migliori per pascolo, ma in generale tutti sono appetiti dal bestiame.

\section{Gen. $9^{\circ}$ - Doryenium Tournf.}

D. herbaceum Vill. (Lotus dorycnium Re-LiN.) Nei pascoli sassosi e caldi.

Per. Giugno, Luglio. (Fiore bianco, carena turchiniccia). Trifoglino it. - Dorycnie herbacée fr.

\section{Gen. 10. - Lotus Lin.}

(Fiori gialli).

L. corniculatus LiN. - Copioso in tutti i prati e pascoli pingui.

Per. Maggio, Settembre. (Vessillo spesso rossegriante). Ginestrina it. - Lotier corniculé fr.

Buono per pascolo.

* $\beta$ humilis Bertol. (Lotus corniculatus alpinus DC. prod.) Cenisio (Bonjean in Bertol.).

* L. uliginosus Schkuhr - Presso i laghi di Avigliana (De Filippi).

Per. Giugno, Agosto.

Loto fistoloso it. - Lotier des marais $\mathrm{fr}$.

(Lotus major Scop.).

Gen. 11. - Tetragomolobue Scop.

-(Fiori gialli, vessillo venato di bruno).

T. siliquosus Rотн, (Lotus siliquosus Re-Lin.).-Frequente nei pascoli umidetti e lungo i fossi.

Per. Maggio, Giugno.

Veccia pisella it. - Tetragonolobe siliqueux fr.

Buono per pascolo. 
B) Foglie imparipennate.

\section{GEN. 12. - Galega LiN.}

G. officinalis LIN. - Galega vulgaris o Ruta capraria Off. - Lungo i fossi dei prati. Rimedio disusato.

Per. Giugno, Luglio. (Fiori cerulei).

Ruta capraria it. - Galega ofincinal, Rue de chèvre fr.

\section{* Gen. 13. - Robimia DC.}

R. pseudo-acacia Lis. - Viali e siepi.

Albero. Maggio, Giugno.

Falsa acacia it. -- Gasia vern. - Robinier faux acacia fr.

Albero bellissimo pei suoi racemi di fiori ordinariamente bianchi, odorosi; è originario dell' America boreale. Cresce rapidamente ed e tenuto in minor conto di quello che merita, poichè quando è adulto presenta un legname bruno, venato di giallo, capace di acquistare col pulimento aspetto bellissimo ed atto per questa ed altre qualità alla costruzione di mobili di lusso. Brucia benissimo anche verde. Le sue foglie non dispiaciono al bestiame.

\section{Gen. 14. - Colutea Lin.}

C. arborescens Lin. - Colutea Off. - Frequente nelle rupi. Le foglie sono succedanee della Senna, ma più deboli.

Arbusto. Giugno, Luglio.?(Fiori gialli).

Erba vescicaria it. - Baguenaudier fr.

Alle Blaccie presso Susa.

Le foglie della Colutea sono purgative e furono chiamate succedanee di quelle della Senna, ma sono disusate nella farmacia moderna. È bene di notare che con esse sovente il commercio sofistica le foglie delle vere specie di Senna. 


\section{Gen. 15. - Phaca Lin.}

* Fiori gialli, o bianchicci.

Ph. alpina $J_{\mathrm{ACQ}}$. - Non rara nei pascoli del Cenisio.

Per. Luglio, Agosto.

Vescicaria gialla it. - Phaque des alpes fr.

Assai appetita dal bestiame.

Nel testo $\mathrm{Ph}$. alpina Lin.

Ph. australis Lin-Mant. - Cenisio.

Per. Luglio, Agosto. (Apice della carena violaceo).

Vescicaria vecciarina it. - Phaque du midì fr.

Cenisio (Negri) (Bonjean in Bertol.) Assietta.

* Ph. frigida Lin., Syst. nat., ed. 10. Altipiano del Cenisio (Negri).

Per. Luglio, Agosto.

Vecciarina pallida it. - Phaque des pays froids fr.

* * Fiori odorosi variopinti bianco-ceruleo-violetti.

Ph. astragalina DC. (Astragalus alpinus RE-LIN.). Sommità alpine di Cesana e del Cenisio.

Per. Luglio, Agosto.

Vescicaria alpina it. - Phaque astragale fr.

Cenisio (Negri) (Balbis, Bonjean in Bertol.) Pascoli del piccolo Moncenisio (Bouvier).

GEN. 16. - Oxytropis DC.

- Fiori gialli o giallastri.

0. pilosa DC. (Astragalus pilosus RE-LiN.). - Nelle alpi di Giaglione e presso Oulx al sito detto il 
Sappé. Nasce anche tra Susa e Bussolino e nella valle di Bardonêche; nè manca nei campi sopra Les Ferrières, ove fu trovata anche da Ignazio Molineri.

Per. Giugno, Luglio.

Astragalina lanuginosa it. - Oxytrope velue fr.

\section{0. campestris DC. (Astragalus campestris RE-LIN.).}

\section{- Copiosa nel Cenisio.}

Per. Luglio, Agosto. (Apice della carena spesso violaceo). Astragalina villosa it. - Oxytrope des campagnes fr.

0. foetida DC. (Astragalus foetidus RE-VILL.). - Co. piosa nel Cenisio.

Per. Giugno, Agosto.

Astragalina vischiosa it. - Oxytrope fétide $\mathrm{fr}$.

Cenisio (Balbis, Bonjean in Bertol.) (Negri).

* Fiori rosei o cerulei.

* O. Iapponica Gaud. - Cenisio dietro l'ospizio (Bonjean in Bertol.). Cenisio (Bouvier) (Negri).

Per. Luglio, Agosto.

Astragalina rosata it. - Oxytrope de Lapponie fr.

* 0. cyanea Breberst. - Cenisio (Bonjean in Bertol.). Altipiano del Cenisio (Negri).

Per. Luglio, Agosto. (Fiori cerulei).

Astragalina azzurra it. - Oxytrope azurrée fr.

0. montana DC. (Astragalus montanus Re-LiN.). Cenisio, alpi di Giaveno.

Per. Luglio, Agosto.

Astragalina montana it. -- Oxytrope des montagnes fr. 


\section{GEN. 17. - Astragalum LiN.}

1) Inermi : fiori porporini violacei, o cerulei.

A. hypoglottis Lin.-Mant. (A. leontinus Re-AlL. auct.)

- Presso la Dora : nel Cenisio.

Per. Giugno, Luglio.

Astragalo serpeggiante it. - Astragale hypoglotte fr.

Cenisio (Bonjean in Bertol.).

Nei prati dietro l'ospizio (Bouvier).

Nel testo: A. leontinus Lin. dal Willd. spec.

A. Onobrychis Lin. - Abbonda nei pascoli : nelle rupi di Monpantero presso Susa, nella contrada detta Madonna della Coà.

Per. Giugno, Luglio.

Cece astragalino it. - A. esparcette fr.

A. austriacus RE-JACQ.-LIN. - Nasce intorno ad Oulx nei siti detti Sappè e Praman.

Per. Luglio, Agosto.

A. d'autriche fr.

Il Bertoloni nella sua Flora italica non riporta questa specie, nonostante ch'essa sia stata notata dall'Allioni, dal Re, dal Colla e da altri autori, che veramente non fecero che eopiare i citati.

Aggiungiamo che il Nyman (Syll. fl. Europ.) non mette l'Astragalus austriacus Lin. (o meglio per priorità A. Austriacus Jacq.) fra le piante italiane.

A. monspessulanus Lin. - In copia nei siti sterili sassosi lungo la Dora presso Susa.

Per. Maggio, Giugno.

Cenisio (Bonjean in Bertol.).

Vecciarino rosato it. - A. de Montpellier fr. 
* A. purpureus Lank. - Altipiano del Cenisio (Negri).

Per. Giugno, Luglio.

Cece montano it. - A. purpre fr.

2) Inermi : fiori gialli, giallastri o bianchicci.

A. Cicer Lin. - Intorno Susa.

Per. Giugno, Luglio.

Cece selvatico it. - A. pois chiche fr.

A. glycyphyllos Lis. - Nei siti incolti.

Per. Giugno, Luglio.

Liquirizia bastarda it. - A. réglisse fr.

* A. depressus Lin. - Cenisio (Bonjean in Bertol.). Per. Giugno, Luglio. (Carena con l'apice purpureo). Astragalo budellino it. - A. nain fr.

3) Spinosi, ciò̀ con pezioli invecchiati persistenti, spiniformi dopo la caduta delle foglioline: fiori rosei o bianchi.

A. aristatus L'Herit. (A. tragacantha Re-AlL. Mise. Taur.) - Gummi tragacantha Off. - Frequente nel Cenisio e nelle alpi di Giaveno: occorre anche nei siti sabbiosi presso la Dora.

Per. Magrio, Luglio.

Dragante bastardo it. - A. à longues dents fr.

La Gummi tragacantha, gomma adragante delle officine, proviene quasi tutta da Astragali esotici, della Persia (Astr. verus Oliv.), della Grecia (Astr. creticus Lamk.), del Monte Libano (Astr. gummifer Labil.).

La farmacia moderna ne fa pochissimo uso. 
Sezione ${ }^{2}$ - Legume diviso brasversalmente in articoli suonosperzi. Cotiledoni, nella germogliazione, fogyliacei, epigei (Fuori terra). Foglie imparipennate. Stami aliadelfi.

A) Fiori in ombrelle.

Gen. 18. - Comomilla LiN.

* Fiori gialli.

C. Emerus Lin. - Nelle selve.

Frutice Maggio, Luglio.

Emero it. - Coronille des jardins fr.

C. minima LiN. - In tutti i pascoli sterili presso Susa.

Per. Luglio, Agosto.

Scorpioide minuta it. - Coronille naine fr.

* C. montana Scop. - Alla Novalesa (Bonjean in Bertol.).

Per. Maggio, Giugno.

Scorpioide montana it. - Coronille de montagne fr.

* Fiori variegati di bianco e di roseo.

C. varia LIN. - Volgare ai margini delle vie e nei campi.

Per. Giugno, Luglio.

Erba ginestrina it. - Coronille bigarrée fr. 


\section{Gen. 19. - IIIppoerepis LIx.}

(Fiori gialli).

H. comosa Lin. - In tutti i pascoli aprici e sassosi.

Per. Maggio, Luglio.

Sferracavallo it. - Hyppocrépide en ombelle fr.

B) Fiori in racemi.

Gex. 20. - Onolbryehis Tourn.

0. sativa LAmk. (Hedisarum onobrychis RE-LIN.) Onobrychis 0ff. - Giallet vern. - Abbonda nei prati. Rimedio disusato.

Per. Maggio, Luglio. (Fiori rosei venati di rosso).

Lupinella; Sanofieno it. - Sanfoin, Herbe éternelle, Esparcette cultivée fr.

La lupinella è mangiata avidamente dal bestiame. $\dot{\mathrm{E}}$ desiderabile che la coltivazione di questa pianta, che resiste all'asciutto e dà un fieno eccellente, sia più diffusa.

Sezione $\mathfrak{S}^{2}$ - Legume uniloculare, foglie paripenuate, terminate da un cirro o da una punta molle spiaiforme, raramente imparipenaate (Gen. Cicer). Cotiledoni, nella germogliazione, farinacei, spessi, ipogei (sotterranei). Stami diadelf, raramente monadelf.

\section{Gen. 21. - Cieer Lin.}

* C. arietinum Lin. - Coltivato.

Ann. Giugno, Lnglio.

Cece it. - Cisi vern. - Pois chiche fr.

$\grave{E}$ da notarsi che Allioni riporta questa specie come spon- 
tanea dei dintorni di Susa (Circa Segusium sponte nascitur, Ped., 1, pag. 332) ed il Re invece non ne fa motto nella sua Flora. 亡̀ peró molto probabile che il Cece non sia spontaneo a Susa ma disseminato per cause meramente accidentali nei siti incolti.

\section{GEN. 22. - Vieia LIN.}

1) Fiori in racemi pit o meno peduncolati.

V. pisiformis Lin. - Nelle selve.

Per. Maggio, Giugno. Fiori giallo-verdognoli).

Veccia pisellina it. - Vesce à feuilles de pois fr.

V. sylvatica Liv. - Nei prati superiori del monte Serre la Garde nella valle di Exilles.

Per. Luglio, Agosto. (Fiori bianchi o violaceo-sbiaditi).

Veccia boschiva it. - Vesce des bois fr.

V. cracca Lrv. - Nei campi e nei prati. Presso Susa a piedi del monte di San Lazzaro.

Per. Giugno, Luglio. (Fiori cerulei).

Cracca it. - Vesce cracca fr.

V. onobrychioides Lis. - Nei prati superiori di Serre la Garde e al Monginevro.

Per. Maggio, Giugno. (Fiori violacei).

Veccia astragalina it. - Vesce fausse esparcette fr.

V. Narbonensis Lin. - Nei prati presso Exilles. Ann. Aprile, Maggio. (Fiori porporini).

Veccia selvatica it. - Vesce de Narbonne fr.

V. sepium LrN. - Margine delle selve: spineti.

Per. Giugno, Luglio. (Fiori cerulei con vene purpuree, raramente bianchi o gialli).

Veccia silvana it. - Vesce des haies fr. 
2) Fiori sessili o quasi, solitari, o a coppie.

V. sativa Lis. - Vessa vern. - Ahi! troppo frequente nelle messi.

Ann. Maggio, Giugno. (Fiori cerulei o violacei).

Veccia buona it. - Vesce cultivée fr.

Il Re si duole che la Veccia invada spesso i seminati, ma la colpa della invasione è dell' agricoltore poco diligente, non già della Veccia, utile pianta che offre nelle sue foglie un pascolo gratissimo al grosso bestiame e nei semi un robusto alimento ai volatili domestici, specialmente ai colombi.

V. angustifolia Rotr. थ. segetalis Kock - (V. angusifolia Re-ALL.). - Nei campi e nei pascoli.

Ann. Maggio, Giugno. (Fiori violacei).

Veccia sottile it. - Vesce à feuilles étroites fr.

V. Iutea Lrs. - Nei pascoli declivi; nella valle d'Exilles tra i macigni presso Jambon e nel monte di San Colombano.

Per. Aprile, Magrgio. (Fiori gialli o purpureo-sbiaditi). Cicerchia pelosa it. - Vesce jaune fr.

V. praecox JACQ. - Nei pascoli declivi. Abbonda nei pascoli aridi di Condove nel sito detto la Rocea. V. lathyroides Lin. - V. minima Riv.

Ann. Marzo, Aprile. (Fiori purpurei o violacei).

Veccia serena it. - Vesce fausse gesse fr.

“V. praecox Jacq. pianta a me ignota omessa dallo Steudel, dal Decandolle e dallo Sprengel. Ebbi però dall' erbario del Biroli una pianta sotto questo nome similissima a Vicia lathyroides ". (Colla Herb. Ped., vol. II, pag. 218).

Aggiungiamo che dall' esemplare mandato da Jacquin ad Allioni col nome di V. praecox, che noi potemmo vedere, 
mercè la gentilezza del prof. Arcangeli, nell'erbario dell'autore della Fl. Pedemontana, risulta che trattasi veramente di una forma di V. lathyroides Lin.

Allioni (Auct. pag. 22) distingue Vicia Lathyroides Lin. dalla pianta descritta ed a lui spedita sotto V. praecox da Jacquin, e molto probabilmente il Re si è tenuto all' opinione dell' Allioni, ch iamando la sua pianta Vicia praecox. È bene notare che il Re nella Fl. Torinese (Vol. II, pag. 14), stampata 20 anni dopo la pubblicazione della Flora Segusiensis, descrive sotto Vicia lathyroides la presunta specie di Jacquin.

\section{* Gen. 23. - Faba Tournf.}

\section{F. vulgaris Moench. - Coltivata.}

Ann. Maggio, Giugno.

Fava it. - Fava vern. - Fère commune fr.

Si dice oriunda delle rive del Caspio, ma veramente, secondo A. Decandolle (Géogrr. bot., v. $2^{\circ}$, pag. 956), sareblee assai dubbio il suo luogo natio primitivo.

È una pianta ricca di materia azotata e costituisce un sano alimento.

\section{Gen. 24. - Ervam Lin.}

\section{E tetraspermum LIN. - Nelle messi.}

Ann. Giugno, Luglio. (Fiori bianchicci, vessillo ceruleo). Tentennino it. - Ers à quattre graines fr.

\section{E. hirsutum LIN. - Ovunque nei campi.}

Ann. Giugno, Luglio. (Fiori bianco-cerulei).

Veccia it. - Ers hérissé fr.

\section{* E. Lens Lin. - Coltivata.}

Ann. Maggio, Giugno.

Lente, Lenticchia it. - Lentie vern. - Lentille fr.

È difficile di stabilire l'origine precisa di questa utilissima specie atteso l'antichità e la diffusione della sua coltura. 


\section{Gen. 25. - Pisum Liv.}

* P. sativum Liv. - Coltivato con parecchie varietà.

Ann. Aprile, Maggio.

Pisello it. - Poïs vern. - Pois fr.

Legume gustoso e nutritivo. Tra le varietà va distinta quella a semi minuti e baccello carnoso commestibile. (Poïs golu vern. piem.).

\section{GEN. 26. - Lathyurus LiN.}

* Picciuolo senza foglioline.

L. aphaca Lin. - Copioso nelle messi.

Ann. Giugno, Luglio. (Fiori gialli).

Veccia bastarda it. - Gesse sans feuilles fr.

- Picciuolo foglioso.

L. hirsutus LiN. - Nei campi.

Bienn. Giugno, Luglio. (Fiori cerulei). Cicerchia pelosa it. - G. hérissée fr.

L. Iatifolius LiN. - Nella valle di Bardonêche e nei prati montani di Gravere nel sito detto del Tat.

Per. Luglio, Agosto. (Fiori rosei).

Cicerchione it. - G. à larges feuilles fr.

L. sphaericus Retz. (L. Coccineus Re-All.) - Comunissimo nei campi.

Ann. Maggio, Giugno. (Fiori rossastri).

Cicerchia scarlata it. - G. sphérique fr. 
L. pratensis LiN. - Nei prati e nei pascoli.

Per. Giugno, Luglio (Fiori gialli; vessillo con vene violacee).

Pisello dei prati it. - G. des prés fr.

Ottimo pascolo come le altre specie.

L. sylvestris Lin. - Presso le selve.

Per. Luglio, Agosto. (Fiori rosei).

Ceserone it. - G. des bois fr.

\section{Gen. 27. - Drobus LIN.}

" Fiori gialli: vessillo spesso rosseggiante.

0. Iuteus Lin. - Nella valle d'Oulx.

Per. Maggio, Giugno.

Galega montana it. - Orobe jaune fr.

* 0. varius Willd. - Nei monti di Giaveno. (Balbis in Bertol.).

Per. Aprile, Maggio. (Vessillo rosseggiante).

Orobo giallo-rossigno it.

(Orobus augustifolius Balbis, misc. alt.).

- Fiori violaceo-purpurei.

0. tuberusus Lin. - Presso le selve.

Per. Aprile, Maggio.

Tartufo di prato it. - Orobe tubéreux fr.

* $\beta$ tenuifolius Koch. (O. tenuifolius Roth.) - Cenisio (Bouvier sotto Lathyrus macrorhizus Wimm.)

0. vernus Lrs. - Orunque nelle selve ombrose. Nelle selve della Bretagna presso Susa.

Per. Aprile, Maggio.

Orobo primaticcio it. - Orobe printanier fr.

0. niger LIs. - Comune nelle selve.

Per. Maggio, Giugno.

Orobo silvano it. - Orobe noir fr. 
Sezione ${ }^{2}$ - Legame bivalve, coi semi divisi da tramezzi cellulosi. Cotiledoni spessi, carnosi, wella germogliazione, epigei (fuori terra). Stami diadelf. Toglie trifogliate.

* Gen. 28. - Pliaseolus Lin.

Ph. vulgaris Lin. - Faseui vern. - Coltivato.

Ann. Luglio, Agosto.

Fagiuolo it. - Haricot fr.

Donde sia venuto in Europa il fagiuolo è molto difficile a sapersi; taluni lo credono originario dell'India o della China meridionale.

Il fagiuolo è un'altra leguminosa assai nutriente da preferirsi ancora alla fava, al pisello ed alle lenticchie, sia perchè di più facile digestione, sia perchè più ricco di materia azotata. Esso merito, o giustamente, il titolo di carne del contadino.

Numerose sono le varieta di fagiuoli coltivate, tutte più o meno pregievoli, tra le quali ra notata quella detta fagiuoli della regina a semi grossi screziati.

Facciamo appena cenno del Dolicos (Dolicos Catiang Lin.). Fagiolino dell'occhio it, a baccello sottile, lungo, cilindrico.

Sotto Ordine $2^{\circ}$ - Stami liberi.

Gen. 29 - Cercis Lin.

\section{Siliquastrum Lin. (Siliquastrum, Arbor ludae Off.). - Presso Susa nei siti scoscesi soleggiati nella contrada detta della Madonna della Consolata. Siccome non si ritrova in un altro sito, dubito che sia indigeno. Rimedio disusato.}


Albero. Maggio.

Siliquastro, Albero di Giuda it. - Cercis gainier, Arbre de Judée fr.

Il Cercis di cui scrive il Re trovasi propriamente nel fondo del sig. avv. Garelli.

Il Siliquastro è indigeno delle parti più australi di Europa: abbonda nel mezzogiorno italiano, ed è a sufficienza giustificato il sospetto dell'autore della Flora Segusina.

\section{Ordine XXXI. - Amiddalee (Amigdalea .Juss.).}

\section{Gex. 1. - Amygdalus Lin.}

\section{A. communis LrN. - A. dulcis o Sativa Off. - Aman-} dole vern. - Così volgare nelle vigne e nelle rupi intorno Susa, che io credo possa ritenersi a buon titolo per indigena. L'olio spremuto dai semi è zutriente, emolliente e alquanto purgativo.

Alberetto. Fior. Marzo, Aprile. - Fruttif. in Agosto.

Mandorlo it. - Amandier fr.

Se il mandorlo sia indigeno ovvero naturalizzato da tempo antico in Italia è assai difficile definire. $\grave{E}$ provato che i Romani l'ebbero dalla Grecia in tempi non molto remoti. locchè farebbe indirettamente argomentare che esso non sia indigeno.

Le mandorle sono nutrienti come sostanze azotate; esse. come l'olio che se ne ricava, sono amministrate in varie forme dalla medicina moderna.

L'olio di mandorla è adoperato anche largamente per uso di profumeria. Le mandorle amare provengono da una varietà dell'A. communis. Esse sono distinte da un odore e sapore speciale di acido cianidrico, il quale fa parte di una sostanza propria delle mandorle amare, l'amigdalina.

La terapia moderna se ne avvale ed il Cantani le annovera tra i rimedi narcotici paralizzanti. 
* Gen. 2. - Persiea Tournf.

\section{P. vulgaris MirL. - Coltivata.}

Alberetto. Fior. Marzo, Aprile. - Fruttif. Luglio, Agosto. Pesco it. - Persi vern. - Pêcher fr.

Pianta originaria dell'Asia, introdotta in Italia dai Romani al principio dell'êra volgare.

Il pesco, di natura polimorfo, obbedì ai comandi dell'uomo e si divise in un grandissimo numero di varietà. Il frutto è sub-acido, rinfrescante.

Lacqua distillata delle foglie e lo sciroppo dei fiori di pesco non sono rimedi disusati, ed agiscono, benchè più debolmente, come i preparati delle mandorle amare.

Gen. 3. - Paumus Tournf.

* P. Armeniaca Lin. - Coltivato.

Arbusto. Fior. Marzo, Aprile. - Fruttif. Giugno, Luglio. Albicocco it. - Arbicoc vern. - Abricotier fr.

Originario dell'Armenia ed in generale della bassa regione caucasica.

* P. domestica Lin. - Coltivato.

Arbusto. Fior. Marzo, Aprile. - Fruttif. Luglio, Settem.

Susino it. - Bërgna vern. - Prunier domestique fr.

Originario della bassa regione caucasica, e polimorfo diede fin dall'antichità un numero considerevole di varietà: ingens turba prunorum, scriveva Plinio fin dai suoi tempi.

Dal P. domestica Koch farebbe derivare tutte le varietà di susine a frutto bislungo.

P. insititia Lrv. - Per testimonianza dell'Allion: trovasi nelle siepi della valle di Bardonêche e di Cesana.

Arbusto. Fior. in Aprile. - Fruttif. Luglio, Settembre.

Prugnolo di siepe it. - Prunier enté fr. 
Originario del Caucaso e probabilmente dei siti temperati dell'Europa australe, questa specie si piegò anch'essa alla coltura e diede, secondo pensa il Koch, le numerose e pregiate varietà di susine a forma rotonda, tra le quali, notis. sima, la susina Reine Claude.

P. spinosa Lin. - P. sylvestris Off. - Bosso vern. Ahi! Troppo comune nelle fratte e nei pascoli. Bacche astringenti.

Arbusto, Aprile.

Susino delle macchie it. - Prunellier fr.

Farmaco disusato.

P. brigantiaca VILL. - Comunissimo nelle siepi fra Oulx e Cesana.

Arbusto, Aprile, Maggio.

Marmotta: Prugno marmotta it. - Prunier de Briaņ̧on fr.

Dai semi del P. Brigantiaca, nella valle d' Culs e in generale nel territorio, che da questa si stende pel Monginevro sino a Briançon, si estrae un olio (olio di marmotta huile de marmote fr.), che è dolce, e serve a quei naturali per condire vivande invece dell'olio di olivo.

\section{* P. laurocerasus Lin. - Coltivato per bellezza} nei giardini.

Lauro ceraso it. - Laurier cerise fr.

Si dice originario dell'Asia minore.

Il frutto del Lauro ceraso si può mangiare impunemente: le foglie sono velenose: l'acqua distillata semplice o concentrata (acqua coobata) di foglie di Lauro ceraso è somministrata tanto internamente che esternamente come antispasmodico. Esse, come i semi di mandorle amare, contengono acido cianidrico. 
* P. avium Lrv. ( $P$. cerasus $\beta$ Bertol. - Cerasus avium Moench). - Coltivato.

Albero. Fior. Aprile. - Fruttif. Giugno, Luglio.

Ciliegio it. - Merisier fr.

\section{$\beta$ juliana Kock. (Cerasus juliana DC.).}

Da questa varietà Kock fa derivare le ciliegie dolci e molli. Ciriegia it. Cerese vern. Guigne, Cerise douce fr.

\section{y duracina Koch. (Cerasus duracina DC.).}

Da questa derivarono, secondo il Koch, le ciliegie dolci ma dure. Ciriegia duracina it. Graffion vern. Bigarreau fr.

\section{P. cerasus Lin. - Nelle selve.}

Albere tto. Fior. Aprile. - Fruttif. Giugno, Luglio.

Ciliegio it. - Cerisier fr. Coltivato nelle sue varietà:

\% acida Koch. - Visciole vern. Gobet fr.

$\beta$ austera Koch. - Prunus austera Ehrh. - Marasca, Amarena it. Griota vern. Griotte fr.

Secondo il Koch. e Alf. Decandolle sareble spontaneo di Europa il P. Avium Lin.; originario del mezzogiorno caucasico, naturalizzato e diventato da lungo tempo spontaneo in Europa il P. Cerasus. Questo sarebbe stato portato da Ceresonte in Italia da Lucullo, generale romano, noto piủ per lussuria e ghiottoneria che per virtù militari e cittadine.

P. Padus Lin. - Cerasus racemosa Off. - Corteccia amara, febbrifuga.

Arbusto. Aprile, Maggio.

Pado it. - Merisier à grappes, Bois joli fr.

Nel testo manca la localita. "Nelle selve e nelle siepi dei luoghi collinosi e montani ". (Allioni, Fl. Ped., vol. II, pag. 134).

Musinè (De-Filippi).

Farmaco disusato. 


\section{P. Mahaleb Lin. - Cerasus amara o Mahaleb Off.}

- Frequente nelle siepi e nei luoghi incolti sassosi soleggiati. Farmaco disusato.

Arbusto. Maggio, Giugno.

Ciliegio canino it. - Cerisier Mahaleb, Bois de Sainte Lucie fr.

\section{Ordine XXXII. - Rosacee (Rosacece Juss.).}

l) Carpelli deiscenti a $2-4$ semi (Follieoli)

talamo concavo.

Gen. $1^{0}$ - Spiraea Lin.

(Fiori bianchi).

- Foglie senza stipule.

S. aruncus Liv. - Nelle fratte e nelle selve. Astringente.

Per. Maggio, Giugno.

Barba di capra it. - Spirée barbe de chèvre fr.

Bella pianta, che spicca pel suo gran racemo terminale a fiorellini odorosi.

Non tanto questa specie quanto le due seguenti furono adoperate come medele astringenti. Analisi recenti scoprirono nelle Spiree l' acido salicilico; sono somministrate come diuretiche in decozioni.

\section{* Foglie stipulate.}

S. ulmaria LIN. - Ulmaria off. - Copiosa nei prati umidi e lung’o i fossi. Virtù della precedente.

Per. Giugno, Luglio.

Olmaria it. - S. Ulmaire, Reine des-prés fr. 


\section{S. filipendula LIN. - Filipendula saxifraga rubra} Off. - Ovunque nei prati secchi e presso le selve. Virtù della precedente.

Per Giugno, Luglio.

Filipendola it. - S. filipendule fr.

2) Carpelli secchi monospermi imaleiscenti (acheni): đaalice 8-9 fido (Bryas), Calice calieulato nelle altre specie.
A) Talamo secco.

GEN. $2^{\circ}$ - Dryas LIN.

(Fiori bianchi: stili persistenti).

D. octopetala Lix. -- Abbonda nelle alpi.

Per. Giugno, Luglio.

Cametrio gentile it. - Dryade à $S$ pétales fr.

Gen. $3^{\circ}$ - Geam Lin.

(Fiori gialli : stili persistenti).

- Canle a molti fiori: stilo articolato.

G. urbanum Lin. - Caryophyllata Off. - Ovunque nei siti incolti ed ombrosi, presso le selve e nelle siepi. Radice subaromatica, tonica-febbrifuga.

Per. Luglio, Agosto.

Cariofillata it. - Benoite, Ilerbe de S. Benoit fr.

Si adopera la radice come tonico ed eccitante degli organi digestivi; come febbrifugo val poco o niente (Cantani).

G. rivale Lin. - Geum rivale o palustre Off. Abbonda nei prati di Bassa Meana e presso i ruscelli ai Cenau e nel Cenisio. Astringente. 
Per. Maggio, Giugno.

Cariofillata acquatica it. - B. des ruisseaux fr.

Farmaco disusato.

" Caule terminato da un solo fiore: stilo non articolato.

G. montanum Lis. - Ovunque nei prati e pascoli montani ed alpini.

Per. Giugno, Agosto.

Cariofillata montana it. - Benoite des montagnes fr.

G. reptans Lis. - Nelle sommità fredde e sassose del Cenisio sovra la Posta: all'Assietta.

Per. Giugno, Agosto.

Cariofillata flagellosa it. - Benoite traçante fr.

Rocciamelone a Casa d'Asti (Sig. ${ }^{2}$ Irene Voli-Chiapusso) ( $\left.{ }^{\star}\right)$.

\section{GEN. $4^{\circ}-$ Siblballa}

(Fiori gialli).

S. procumbens Lin. - Siti freddi, umidetti del Cenisio.

Per. Luglio, Agosto.

Fragola matta it. - Sibbaldie couchée fr.

\section{Gen. 50. - Potemtilla Lin.}

1) Fiori bianchi.

* Foglie 3-fugliate

P. fragariastrum Eнrн. (Fragaria sterilis Re-LiN.). Per. Aprile, Maggio.

Fragola secca it. - Potentille fraisier fr.

(") Questa distinta signora, degna consorte del cav. Felice Chiapusso, ha ritratto egregiamente sul posto e dal vero parecchie piante alpise : e noi siamo lieti di poter registrare in queste pagine, insieme al suo nome, varie località non menzionate dal Re. 
* Foglie radicali ¿-digitate.

P. caulescens Lin. - A Susa sulla rupe detta di Crofassé, e tra i sassi sul Cenisio presso l' $\mathrm{F}_{\text {- }}$ chelle.

Per. Luglio, Agosto.

Potentilla penzola it. - Potentille ascendante fr.

P. alba LiN. - Abbonda nei castagrneti di Mocchie: comune anche altrove nei pascoli montani e collinosi presso le selve.

Per. Maggio, Giugno.

Pentafillo bianco it. - P. blanche fr.

* Foglie radicali pennate.

P. rupestris LIN. - Nei prati montani e declivi. Per. Maggio, Luglio.

Fragolaccia rupina it. - P. des rochers fr.

2) Fiori gialli.

- Foglie radicali pennate.

* P. multifida Lin. - Cenisio presso l'O Ospizio (Bouvier) - Cenisio (Bonjean in Bertol.).

Per. Luglio, Agosto.

Potentilla pennata it. - P. decoupée fr.

* Foglie 3-fogliate.

* P. nivea Lrs. - Cenisio (Bouvier). - Alpi di Oulx (Moris in Bertol.).

Per. Luglio, Agosto.

Fragola canda it. - P. blanc de neige fr.

Pianta nana elegantissima, peziolo corto a tomento niveo. 
* P. frigida Vill. - Cenisio a Ronche (Bouvier)

- Cenisio (Bonjean in Bertol.).

Per. Luglio, Agosto.

Fragolina barbuta it. - P. des frimas fr.

* P. minima Haller fil. - Cenisio a Ronche (Bouvier) - Cenisio (Bonjean in Bertol.).

Per. Luglio, Agosto.

Fragolina piccina it. - P. naine fr.

P. grandiflora Lin. - Nei pascoli alpini.

Per. Luglio, Agosto.

Fragolaccia trifogliata it. - P. ̀̀ grandes fleurs fr.

P. tormentilla Siвthorp. (Tormentilla erecta RE-Lin.). - Tormentilla Off. - Non rara nei siti montani presso le selve. A Susa lungo i rivoli di Monpantero superiore. Tonica, astringente.

Per. Maggio, Settembre.

Tormentilla it. - Tormentille dressée fr.

La radice della $\mathrm{P}$. tormentilla contiene molto tannino ed è ottimo rimedio astringente; può sostituire la radice di Ratania, che non sempre si trova buona (Cantani).

*** Foglie radicali 5-7 digitate:

le cauline conformi o spesso ternate.

P. recta. - Nei pascoli.

Per. Luglio, Agosto.

Cinquefoglio it. - P. dressée fr.

P. argentea Lin. - Nei pascoli sterili e sassosi. Per. Maggio, Luglio.

Cinquefoglio bianco it. - P. argentée fr. 
P. hirta Lin. - Colli aridi delle Blaccie, lato meridionale della Brunetta.

Per. Maggio, Giugno.

Fragolaccia rossa it. - P. hérissée fr.

P. aurea Lin. - Siti freddi ed umidetti delle alpi.

Per. Luglio, Agosto.

Fior d'oro it. - P. dorée fr.

Cenisio (Bonjean in Bertol.).

* P. alpestris Haller fil. - Altipiano del Cenisio (Negri).

Per. Luglio, Agosto.

Eragolaccia alpestre it. - P. des alpes fr.

Questa specie è riportata, e forse a ragione, dal Bouvier col nome di P. maculata Pourret, poichè con questo nome la descrisse il Pourret 30 anni prima di Haller fil.

Potrebbe invero per la ragione stessa portare anche il nome di P. Salisburgensis Haenk, poichè questo botanico (in Jacq. collect.) la descrisse nell'anno medesimo (1788) in cui fu descritta dal Pourret (Bouvier).

P. Verna Lin. - Nelle rupi, specialmente nei monti di Bussolino presso la borgata detta dei Gonté.

Per. Aprile, Maggio.

Fragolaccia primaticcia it. - P. du printemps fr.

$\beta$ hirsutissima Bertol. (P. opaca Re-All. non LiN, secondo Bertol.) - Comune nei pascoli e nelle rupi presso Susa.

Per. Maggio, Giugno.

“Questa varietà è la stessa specie, ma irsutissima, spe- 
“ cialmente nel caule. Trovi individui intermedii che non " sai se rapportarli alla specie o alla varietà. P. opaca " Lin. differisce di gran lunga da questa varietà, nè sinora " fu raccolta in Italia." (Bertoloni, Fl. it., vol. V, pag. 280). Senza occuparci se la specie Linneana sia stata raccolta in Italia dopo la pubblicazione della Flora del Bertoloni noi crediamo, per quanto abbiamo potuto osservare e riscontrare, che il Bertoloni opini rettamente circa P. opaca del Re e degli autori non recenti piemontesi.

\section{P. reptans Liv. - Quinquefolium o Pentaphyllum} Off. - Frequente nei prati e nei pascoli umidetti. Radice astringente.

Per. Giugno, Luglio.

Cinquefoglio it. - P. rampante fr.

Le radici della $P$. reptans e dell 'Argentea hanno un'azione pressochè simile a quella della Tormentilla.

B) Talamo spugnoso-sub-carnoso.

\section{Gen. 6. - Comarum Lin.}

(Fiore di un rosso cupo).

C. palusire Lis. - Siti umidi alpini.

Per Giugno, Luglio.

Cinquefoglio di palude it. - Comaret des marais fr.

C) Talamo carnoso-succulento.

\section{Gen. 7. - Fragaria Lin.}

F. Vosca Lin. - F. Off. - Frola vern. - Ovunque nei pascoli e nei siti incolti. Radice diuretica, frutto refrigerante.

Per. Maggio, Giugno.

Fragola selvatica it. - Fraisier commun fr. 
Le cosidette fragole Ananas coltivate in giardino provengono dalla Fragaria grandiflora e Chilensis Ehrh.

Le fragole contengono acido citrico e malico, sali vegetali, zucchero ed un olio volatile, al quale devono il loro piacevole sapore (Cantani).

Hanno azione diuretica e purgativa moderata, e la radice agisce come astringento e diuretica; questa fu vantata contro il colèra.

3) Carpelli suceulenti (drupe)

aggregati su talam piti meno convesso :

calice non caliculato.

Gen. 8. - Medoudes LiN.

* Caule fruticoso.

R. idaeus LrN. e Off. - Àmpole vern. - Frequente nei monti di Sant'Antonino, nel Cenisio. Fruiti acidulo-dolci, rinfrescanti.

Per. Maggio, Giugno.

Lampone it. - Framboisier fr.

Colle della Russa nei monti di Giaveno.

Il lampone contiene come le fragrole acido citrico e malico, ecc., ed un olio etereo che gli dà l'odore ed il sapore speciale. E diuretico e leggermente purgativo (Cantani).

R. fruticosus Lin. - R. Off. - Ronse, More vern. - Volgare nelle siepi. Frutti subacidi rinfrescanti.

Per Giugno, Settembre.

Rovo it. - Ronche arbrisseau fr.

Le foglie del R. fruticosus, come tanniche, sono astringenti; il frutto (le more) sono acidule e rinfrescanti.

R. coesius LiN. - Ovunque presso le acque.

Per. Luglio, Agosto. (Frutto verde pallido).

Rovo di fior bianco it. - More vern. - Ronche à fruit bleuâtre fr. 
* Caule erbaceo.

R. saxatilis Lin. - Nel Cenisio e nei monti di Meana presso il villaggio delle grandi Traverse. Per. Maggio, Luglio.

Rovo erbaiolo it. - Ronche des rochers fr.

4) Carpelli indeiscenti (acheni) rinchinsi nel tubo del calice fatto al orcialo, alla matsoiti carmoso e succulento. Calice non caliculato.

\section{Gen. $9^{\circ}$. - Rosa Lin.}

(Frutici).

1) Stili strettamente riuniti in una colonna centrale.

R. arvensis LiN. - Nei pascoli.

Maggio, Giugno. (Fiori bianchi).

Rosa corallina it. - Rosier des champs fr.

2) Stili liberi.

* Frutto globoso.

a) Foglie seghettate a denti semplici.

\section{R. spinosissima Lin. - Cenisio.}

Giugno, Luglio.

Rosa di macchia it. - R. pimprenelle fr.

R. cinnamomea Lin. - Trovasi, sulla fede di Allioni, nelle selve di monte Chapé presso Exilles.

Giugno, Luglio.

Rosa cannella it. - R. cannelle fr.

Specie assai dubbia per lo meno come pertinente alla flora di Susa. Il Re la riporta sulla fede dell' Allioni; anzi è da notar questo, che non fu l'Allioni che la ritrovò nella località sovra accennata, ma D. Valle (D. Valle reperit in sylvis etc. All., Fl. Ped., vol. II, pag. 138); nè si rinviene nell'erbario del- 
l'Allioni alcun esemplare di questa specie da lui raccolto in Piemonte, o ricevuto da altri siti, nonostante che il Bertoloni scriva (Fl. it.) che in detto erbario se ne trovi uno di provenienza svizzera.

Il Bertoloni a questo proposito scrive " (Fl. it., vol. 5, pag. 215) che la R. cinnamomea è coltivata nei giardini, ma che egli non la vide mai, nè l'ebbe da altri come spontanea.

b) Foglie seghettate a denti seghettati.

* R. pomifera Smith. - Cenisio al Morallet (Bonjean in Bertol.).

Giugno, Luglio.

Rosa pomifera it. $-R$. pomifère fr.

(Rosa villosa All.).

* * Frutto bislungo.

a) Foglie seghettate a denti semplici.

R. canina LiN. - Cynorrhodon Off. - Gratacuj vern. - Ovunque nelle siepi e nelle selve.

Rosa selvatica it. - R. des chiens, Errlantier sauvage fr.

Il frutto della $R$. canina era adoperato sotto forma di conserva (conserva di Cynorrhodon) come astringente nei profluvii intestinali. L' acqua di Roselline delle farmacie si ottiene mediante la distillazione dei petali ed è leggermente astringente.

b) Foglie seghettate a denti seghettati.

* R. sepium Thuil. - R. rubiginosa Lin. Mant. - R. rubiginosa $\beta$ Bertol.). - Cenisio al Morallet verso Susa (Bonjean in Bertol.).

Maggio, Giugno.

Rosa da siepe it. - R. rouillé fr. 
R. pumila LIN. fil - Nei pascoli montani soleggiati.

Maggio, Giugno.

Rosa serpeggiante it. - R. nain fr.

R. alpina Lin. - Nel Cenisio.

Giugno, Luglio.

Rosa alpina it. - R. des alpes fr.

Musinè (Re, Fl. Tor.). Alpi di Novalesa nella contrada detta Tourdupi (Signora Chiapusso-Voli).

R. glandulosa Belz. - Nei pascoli alpini. Trovata dal chiar. Bellardi nelle selve del Cenisio, presso il lago.

Giugno, Luglio.

Secondo Bertoloni questa specie sarebbe la stessa R. alpina Lin. in istato di minor sviluppo.

Giova a questo proposito accennare che le rose occupano uno dei primi posti nella serie delle piante polimorfe e quindi dai botanici furono suddivise in un numero considerevole e spaventoso di specie e di varietà. Nei giardini sono coltivate per bellezza varie specie di Rose nostrane e molte straniere note a tutti.

5) Carpelli índeiscemi (acheni) imanersi mel tubo del eallee alla maturità ind manca la corolla.

Gen. 10. - Amoimomia Lin.

A. Eupatoria Lin. - Eupatorium veterum o Agr . monia Oíf. - Agrimonia vern. - Frequente nei pascoli secchi e nelle siepi. Subastringente.

Per. Giugno, Luglio.

Eupatoria it. - Aigremoine Eupatoire fr. 


\section{GEN. 11 - Mchemilla LiN.}

* Fiori in corimbi terrninali.

A. vulgaris Lin. - A. stellaria o Pes Leonis Off. - Presso Meana. Frequente lungo i rivoli del Moncenisio e nei monti di Giaveno. Fusto e radici astringenti.

Per. Maggio, Luglio.

Alchemilla, Piè di Leone it. - Alchemille commune fr.

A. alpina Lrv. - A. argentea Off. - Prati e pascoli alpestri.

Per. Giugno, Agosto.

Erba ventaglina it. - Alch. des alpes fr.

A. pentaphyllea Lin. - Nei pascoli freddi ed umidetti del Cenisio.

Per. Luglio, Agosto.

Ventaglina sbrandellata it. - Alch. à cinq feuilles fr.

Cenisio (Balbis, Bonjean in Bertol.) Cima d' Eau blanche (Negri). Specie alpina non comune.

** Fiori ascellari agglomerati.

A. arvensis Scop. (Aphanes arvensis Re-Lin.) - Perchepier o Alchemilla minima Off. - Qua e là nei campi. Rimedio disusato.

Ann. Giugno, Luglio.

Ventaglini it. - Alch. des champs fr.

GEN. 12. - Samguisortha Lin.

S. officinalis Lin. - Pimpinellae sanguisorbae radix Off. - Frequente nei prati umidi. Astringente, corroborante. 
Per. Giugno, Agosto.

Sanguisorba it. - Sanguisorbe officinale fr.

Gen. 13. - Potenium LiN.

P. sanguisorba Liv. - Pimpinella rubra Off. Frequente nelle vigne. Astringente.

Per. Maggio, Giugno.

Salvastrella minore it. - Pimprenelle sanguisorbe fr.

Tanto le specie di Alchemilla, come la Sanguisorba e il Poterium contengono acido tannico e sono astringenti; ma il loro uso nella farmacia moderna è molto limitato, almeno in Italia.

Ordine XXXIII. - Pomifere (Pomaceae Juss.).

Gen. $1^{0}$ - Crataegras Lin.

C. Oxyacantha Lin. - Oxyacantha Off. - Buss bianc vern. - Volgarissimo nelle siepi e nelle fratte. Rimedio disusato.

Frutice Aprile, Maggio.

Bianco spino it. - Aubepine commune fr.

Gen. $2^{\circ}$ - Cotomeaster Medik.

C. vulgaris LindL. (Mespilus cotoneaster RE-Lin.) Frequente nei pascoli del Cenisio.

Frutice Maggio.

Cotognastro it. - Cotonnier commun fr.

Altipiano del Cenisio (Negri).

* C. tomentosa Lindu. - Ad Oulx. (Balbis, Elenc. sotto Mespilus tomentosa).

Frutice Aprile, Maggio.

Cotognastro tomentoso it. - C. laineux fr. 


\section{GEN. $3^{\circ}$ - Mespilus LiN.}

M. germanica Lin. - Mespilus Off. - Nelle selve. Frutto sub-astringente.

Arbusto. Maggio, Giugno.

Nespolo it. - Nespo vern. - Neflier d'Allemagne fr.

È coltivato.

Farmaco disusato.

\section{Gen. 4. - Pyrus Lin.}

* Stili liberi.

P. communis LiN. - Prussè vern. - Qua e là nelle selve dei colli e nei siti sterili montani. Albero. Aprile, Maggio.

Pero selvat. it. - Pruss vern. il frutto-Poirier commun fr. Da questa specie derivarono le numerosissime varietà di peri coltivati.

* Stili congiunti alla base.

* P. malus Lin. - Coltivato.

Albero. Aprile, Maggio.

Melo it. - Poumè vern. - Poum, il frutto - Pommiergfr.

Da questa specie, ch'è indigena, derivarono, come dalla specie antecedente, molteplici varietà, a frutta di varia grandezza e più o meno pregiate.

\section{Gen. 5. - Aromia Pers.}

A. rotundifolia Pers. Косн. (Mespilus Amelanchier Re-Lin.) - Malagne vern. - In copia sopra Foresto e nelle rupi intorno Susa.

Arbusto. Aprile, Maggio.

Pero corvino it. - Amelanchier commun fr.

Alberetto di effetto bellissimo quando è in fiore. 


\section{GEN. 6. - Gorbus LIN.}

* Petali patenti bianchi.

S. aucuparia Lin. - Frequente nelle selve montane ed alpine.

Albero. Maggio, Giugno.

Sorbo selvatico it. -- Tumel, Trèmo vern. - Sorbier des oiseleurs fr.

Albero oltremodo elegante pel suo fogliame pennato, per la sua inflorescenza in corimbi composti grandi e patenti e per le frutta di color cinabro vivo.

Il legname del S. aucuparia è durissimo e si adopera, fra gli altri lavori, per costruire viti da torchio.

S. aria Crantz. (Crataegus aria Re-Lin.) - In tutti i monti.

Arbusto. Giugno.

Sorbo montano it. - S. alisier fr.

\section{* Petali eretti rosei.}

S. chamaemespilus Crantz. (Mespilus chamaemespilus Re-Lin.). - Siti freddi del Cenisio.

Frutice Giugno.

Salciagnolo it. - Amélanchier faux néflier fr. Cenisio (Bonjean in Bertol.)

Ordine XXXIV. --. Onagrarie (Onagrariae DC: Gen. 1. - Epildoism Lin.

(Fiori rosei porporini raramente bianchi).

* Petali interi : stilo e stami inclinati.

E. augustifolium Lrs. - Trovato da Ignazio Molineri nei monti di Giaveno e da me nelle rupi presso la chiesetta della Madonna della Losa. 
Per Giugno, Agosto.

Erba S. Antonio it. - Epilobe à épis fr.

Camposanto di Forno dopo Coazze: Musinè.

(E. spicatum Lamk.).

E. Dodonoei Vill. - Frequente nelle alpi e lungo la Dora ed i torrenti.

Per. Luglio, Settembre.

Ramerino di fiume it. - E. à feuille de romarin fr.

* E. Fleischeri Hochst. - Cenisio (Bonjean in Bertol.). Località medesima (Bouvier).

Per. Luglio, Agosto.

Ramerino alpino it. - E. de Fleischer fr.

** Petali bilabati: stilo e stami eretti.

a) Stigmi patenti.

E. hirsutum Lis. (E. grandiflorum RE-ALr.). - Qua e là nei siti umidi ed acquosi.

Per. Giugno, Luglio.

Garofano d'acqua it. - E. hérissé fr.

E. parviflorum Schreb. (E. hirsutum Re $\beta$ LIN.). Ovunque presso i fossi e nei luoghi bagnati.

Per. Luglio, Agosto.

Garofanini d'acqua it. - E. mollet fr.

E. montanum Lrs. - Nei siti ombrosi ed umidetti.

Per. Giugno, Agosto.

Garofanini di montagna it. - E. des montagnes fr.

b) Stigmi riuniti a forma di clava.

E. tetragonum Lis. - Siti medesimi della specie antecedente.

Per. Giugno, Luglio.

Sfenice selvatica it. - E. tétragone fr. 
E. alpinum Lin. - Presso i rivoli freddi del Cenisio.

Per. Luglio, Agosto.

Violine d'alpe it. - E. des alpes fr.

*E. origanifolium Lamк. - Cenisio (Bonjean in Bertol.).

Per. Luglio, Agosto.

Violine basilichine it. - E. à feuilles d'origan - (E. alsinefolium Vill.).

\section{Gen. 2. - Demotheren Lin.}

(Fiori gialli).

Oe. biennis Lin. - Nei pascoli sabbiosi.

Bienn. Giugno, Agosto.

Enagra it. - Onagre bisannuelle fr.

\section{* Gen. 3. - Ismardia LIN.}

I. palustris LiN. - Attorno ai fossi e nelle acque stagnanti a Caselette. (De Filippi).

Per. Luglio, Agosto.

Porracchia di fosso it. - Isnardie des marais fr.

\section{Gen. 4. - Cincaea Lin.}

(Fiori bianchi o rosei).

C. Iutetiana Lin. - Nei siti montani ombrosi ed̀ umidetti. - Frequentissima.

Per. Giugno, Agosto. (Fiori senza brattee).

Erba maga it. - Herbe aux sorcières fr.

C. alpina Lin. - Nel Cenisio.

Per. Giugno, Agosto. (Ficri con piccole brattee). Circea montana it. - Circée des alpes fr. 


\section{Ordine XXXV. - Aloragee.}

\section{(Halorageae R. Brown).}

GeN. UNico. -- Myrioplhyllum LIN.

M. spicalum LiN. - Nelle acque presso le sorgenti ed ai laghi d'Avigliana.

Per. Giugno, Settembre.

Millefoglio d'acqua it. - Volant d'eau en épi fr.

Ordine XXXVI. - IppunIDEe. (Hippurideđe Link.).

GeN. unico. - Mippuneis LiN.

H. vulgaris Lin. - Nelle acque a lento corso.

Per. Luglio, Agosto.

Coda di cavallo acquatica it. - Pesse commune fr.

Ordine XXXVII. - CALlitrichine. (Callitrichineae Link).

Genere unico. - Callitnielne Lin.

C. verna Lin. - Nei ruscelli presso le sorgenti. Ann. Giugno, Luglio.

Erba gamberaia it. - Callitrique printanière fr.

C. autumnalis LiN. - Siti bagnati.

Ann. Agosto, Settembre.

Erba gamberaia it. - C. d'automne fr. 
Ordine XXXViII. - Ceratofillacee.

(Ceratophylleae Gray.)

Gen. unico. - Ceratophyllum Lin.

C. demersum Lis. - Laghi d'Avigliana.

Per. Luglio, Agosto.

Coda di volpe it. - Hydre cornu fr.

Ordine XXXIX. - Litrarie. (Lythrarieae Juss.)

Gen. 1. - Lythrenm Lin.

(Fiori porporini).

L. salicaria LrN. - Nei margini dei fossi e nei prati umidi.

Per. Luglio, Settembre. (Foglie orato-laureolate).

Salcerella it. - Salicaire commune fr.

L. hyssopifolia LiN. - Lungo la Dora.

Ann. Luglio, Settembre. (Foglie lineari od oblunghe). Correggiola dei fcssi it. - S. à feuilles d'hyssope fr.

\section{GEN. 2. - Peplis Lin.}

(Petali rosei sfesso mancanti).

P. portula LiN. - Nei pascoli umidi.

Ann. Giugno, Settembre.

Erba portula it. - Péplide pourpier fr. 


\section{Ordine XL. - TAMariscineE.}

\section{(Tamariscineae Desv.) \\ Gen. Myricaria Desv.}

M. germanica Desv. (Tamarix germanica Re-LiN.). - Copiosa presso la Dora e dietro la Brunetta in siti sabbiosi.

Frutice Maggio, Giugno. (Fiori rosei).

Tamerice it. - Myricaire germanique fr.

\section{Ordine Xli. - Cucurbitacee.}

(Cucurbitaceae Juss.)

\section{GeN. 1. - IBryomia LIN.}

B. dioica JACQ. (B. alba RE-AlL.). Bryonia o Vitis alba Off. - Coussa salvaia vern. - Negli spineti. Radice drastica.

Per Giugno, Luglio.

Barbone it. - Bryone dioïque fr.

Nel testo B. alba Lin. dal sinonimo di Allioni.

La radice di Brionia agisce come drastico forte. Si usa in medicina come purgante nei casi di stitichezza e di stasi nella vena Porta, e quindi anche in varie malattie epatiche.

Il principio attivo della Brionia è riposto in una sostanza estrattiva solubile, Brionina (Cantani).

\section{* Gen. 2. -- Cucandula Lin.}

C. pepo Lin. - Coltivata.

Zucca it. - Cousse vern. - Courge fr.

Numerose sono le varietà di zucche a carne bianca o gialla, a forme bislunghe o rotonde. I Coussot vern. appartengono alla varietà verde quarantina. 
Il luogo originario di questa specie è incerto. Alf. Decandolle nella sua grande opera (Geog. Botanique), dopo lungo ragionare su questo soggetto, conchiude che la patria di essa sembra che sia l'Asia meridionale.

La zucca vuota, zucca da vino, coussa da vin vern., ̀̀ Cucurbita lagenaria Lin. Fu detto che questa specie sia d'origine americana, ma il Decandolle nell'opera sopra citata combatte questa opinione, e sostiene con validi argomenti che sia invece nativa dell'Asia.

\section{* Gen. $3^{\circ}$ - Cureumis Lin.}

C. Sativus Lin. - Dell'Asia. Coltivato.

Ann. Maggio, Giugno.

Citrullo it. - Cocomer vern. - Cornichon fr.

\section{C. melo LiN.}

Popone: Melone it. - Melon vern. - Melon fr.

Forma nella Provincia di Torino un ramo di coltivazione assai proficua. Nel Circondario di Susa è però pochissimo coltivato.

Chiudiamo questo breve cenno sulle Cucurbitacee coltivate col notare che i semi della zucca sono stati adoperati con successo, se non pieno almeno soddisfacente, contro la tenia, e in generale contro l'elmintiasi. Gioverebbero pure come antelmintici i semi del citrullo. Martin sostiene che per ottenere lo scopo bisogna mangiarli con tutto il guscio (Cantani).

Ordine XLII. - Portulacee (Portulaceae Juss.). Gen. $1^{0}$ - Fordulaca LiN.

P. oleracea Lin. - Portulaca Off. - Pourslana vern. - Comunissima negli orti. Refrigerante: coagula il latte. 
Ann. Giugno, Settembre.

Porcellana it. - Pourpier cultivé fr.

Farmaco disusato: non sapremmo che dire circa la proprietà di coagulare il latte attribuita dal Re alla Porcellana. Certo è che oggi non è usata, e da pochi, che come erba da insalata.

\section{Gen. $2^{\circ}$ - MIomtia Lin.}

M. fontana Lin. - Copiosa presso le fonti nei monti di Giaveno.

Per. Maggio, Agosto.

Pendolino it. - Montie des fontaines fr.

Sagra di S. Michele (De-Filippi).

Ordine XLiII. - Paronichiee (Paronychiene Saint Hil.)

$$
\text { Gen. } 1^{\circ} \text { - Teleplnium Lin. }
$$

T. Imperati Lin. - Nelle vigne presso Susa ed anche presso Giaveno.

Per. Giugno, Luglio.

Telefio it. - Télèphe d'Impérati fr.

Alle Blaccie e presso Gravere (Negri). - Dintorni di Susa. (Re, Bonjean in Bertol.).

\section{Gen. $2^{0}$ - Hermianoia LiN.}

* Foglie del tutto glabre, o cigliate (H. alpina).

H. glabra Liv. - Herniaria o Herba turca Off. Nei siti sabbiosi e nelle vigne intorno Susa. Farmaco disusato.

Per. Giugno, Luglio.

Erniola it. - Herniaire glabre fr. 


\section{H. alpina Will. - Nel Cenisio.}

Per. Luglio, Agosto.

Lenticchina d'alpi it. - H. des alpes fr.

Cenisio a Ronche (Bonjean e Re in Bertol.).

* * Foglie irsute.

H. hirsuta Lin. - Nella valle d'Exilles e alla Madonna della Coà presso Susa.

Per. Maggio, Luglio.

Renaiola pelosa it. - H. velue fr.

* H. incana Lamk. - Su la Brunetta (Negri). - Alla Madonna della Coà presso Susa (Re, App. IV, inedita in Colla, Herb. Ped., vol. VII, pag. 407).

Per. Maggio, Giugno.

Erniola bianchiccia it. - H. blanchâtre fr.

\section{Gen. $3^{\circ}$ - Promyellaia Tournf.}

P. argenteaLamk. (Illecebrum paronychia RE-LiN.) Siti sterili.

Per. Aprile, Maggio.

Paronichia argentina it. - Paronyque argentée fr.

P. capitata Lamk. (IIlecebrum capitatum Re-Lin.). Luoghi arenosi montani e caldi.

Per. Maggio, Giugno.

Paronichia sermolina it. - P. en tête fr.

Rupi apriche di Bardonêche presso Millaures.

P. polygonifolia DC. (Illecebrum polygonifolium REBelz.). - Trovata dal chiar. Bellardi nei siti sabbiosi del Cenisio.

Per. Maggio, Giugno.

P. centinodia it. - P. à feuilles de renouée fr. 


\section{GEN. $4^{0}$ - Ortegia.}

O. dichotoma Lin. MANT. - Presso Giaveno.

Per. Maggio, Giugno.

Ortegia alsinella it.

$\mathrm{Da}$ Giaveno (Molineri e Balbis in Bertol.).

Il ch. si gnor Francesco Negri ci scrive che questa specie non si rinviene più nella località, unica in Italia, accennata dall'Allioni (Ped.) e dal Re, per quante ricerche vi abbiano fatte parecchi botanici moderni, fra i quali il dott. Rostan.

Gen. $5^{\circ}$ - Polyeanpom Lin.

P. tetraphyllum LiN. fil. - Siti ombrosi.

Ann. Luglio, Settembre.

Erba migliarina it. - Polycarpe à quatre feuilles fr.

Ordine XLIV - Sclerantee (Scleranthede Link.).

Gen. Unico - Selleramthus Lin.

S. annuus LiN. - Nei campi : abbonda nell'agro di Condove nei siti aprici della contrada detta la Roccea andando verso la casa di S. Pietro.

Ann. Giugno, Settembre.

Centigrani it. - Gnavelle annuelle fr.

Ordine XlV. - Crassulacee (Crassulaceae DC). GEN. $1^{\circ}$ - Rhodiolla Lin.

R. rosea Lrn. - Alpi di Giaveno e di Villarfocchiardo - Cenisio. 
Per. Luglio, Agosto.

Radice idea it. - Rhodiole à odeur de rose fr.

Intorno e dentro le antiche fortificazioni del Colle delle Finestre.

\section{Gen. $2^{\circ}$ - Crassula Lin.}

C. rubens Lin. Syst. nat. - Nei pascoli alpini e declivi di Bussoleno e di Chianoc, ecc.

Ann. Maggio, Giugno.

Vermicolare stellata it. - Crassule rougeâtre fr.

\section{Gen. $3^{\circ}$ - Sedium DC.}

1) Foglie piane, larghe.

\section{S. Iatifolium Bertol. (S. Telephium Re ò $\approx$ LiN.)}

- Frequente presso le vigne.

Per. Agosto, Ottobre. (Fiori verdognoli).

Erba da calli it. - Orpin à larges feuilles fr.

S. Anacampseros Liv. - Nei luoghi montani ed alpini.

Per. Luglio, Agosto. (Fiori purpurei).

Anacampsero it. - 0 . anacampseros $\mathrm{fr}$.

2) Foglie sub-cilindriche, o sub-ovate.

a) Fiori bianchi o rosei.

S. dasyphyllum Lin. - Nei sassi presso le vigne. Per. Giugno, Luglio.

Erba della madonna it. - 0 . à feuilles épaisses fr.

S. hirsutum ALL. - Trovato da Ignazio Molineri nei monti di Giaveno. Si rinviene pure presso Mocchie. 
Per. Giugno, Luglio.

Sedo irsuto it. - 0 . herissé fr.

Pel Bertoloni questa specie è una semplice forma della precedente.

\section{S. atratum LiN. - Cenisio.}

Ann. Luglio, Agosto.

Vermicolare sanguigno it. - Orp. noirâtre fr.

S. album Lin. - Frequente nelle mura delle vigne.

Per. Giugno, Agrosto.

Erba grassa it. - Orp. blanc fr.

b) Fiori gialli.

S. acre Lin. - Frequente nei pascoli umidi alpini.

Per. Giugno, Luglio.

Semprevivi acre it. - Orp. acre fr.

S. alpestre VıL. (S. saxatile RE-ALL.). - Frequente nel Cenisio.

Per. Luglio, Agosto.

Vermicolare alpina it. - Orp. des alpes fr.

(S. repens Schleich. Koch).

S. annuum LiN. - Fra i sassi nelle alpi di Chianoc e del Cenisio.

Bienn. Giugno, Agosto.

Vermicolare alpina it. - Orp. des alpes fr.

Musinè (De Filippi) (Sedum aestivum All. sec. Koch.)

S. reflexum Lin. - Ovunque nei siti sassosi.

Per. Luglio, Agosto.

Sopravivolo de' muri it. - 0 . reflechi fr. 
S. sexangulare LIN. - Quasi in tutti i pascoli sterili muscosi.

Per. Giugno, Luglio.

Erba pignola it. - 0 . à six angles fr.

GeN. 4. - Sempervivam LiN.

* S. tectorum LiN. - Musiné. (Re, Fl. Tor.). Novalesa, contrada Tourdupi (signora ChiapussoVoli). Alle Blaccie.

Per. Giugno, Agosto. (Petali roseo-sbiaditi).

Semprevivo maggiore it. - Joubarbe des toits fr.

S. Wulfenii Hopp. (S. globiferum RE-JACQ.). - Nelle rupi intorno a Susa nel luogo detto Monpantero il Vecchio.

Per. Luglio, Agosto. (Petali citrini).

Sopravivolo giallo it.

Alpi di Thouilles e di Bruzzolo sotto la punta Cruvin. (Sig. Lepetit Dollfus) (*).

Nel testo S. globiferum Lin. dal sinonimo di Allioni.

S. montanum LiN. - Musiné ed altri siti montani.

Per. Luglio, Agosto. (Petali rosei).

Guarda casa it. - J. de montagne fr.

S. aracnoideum LiN. - Pascoli sassosi montani ed alpini.

Per. Luglio, Agosto. (Petali rosei).

Sopravivolo ragnatello it. - J. à toile d'araignée fr.

(") Distinto amatore di botanica e Condirettore della Sezione del Club Alpino di Susa. 
Ordine XLVI. - Grossulariee. (Grossularieae DC).

\section{Gen. unico. - Ribes Lin.}

(Frutici).

- Peduncoli a 1-3 fiori: cauli spinosi.

R. grossularia Lin. - Sopra Les Ferrières.

ß. pubescens Kock (R. uva crispa RE-Lin.). - Raccolta da me nei luoghi sassosi verso Venaus scendendo dalla Brunetta, Bacche acido-dolci.

Maggio.

Uva spina it. - Grisela vern. -- Groseiller epineux : Groseiller maquereau fr.

-* Fiori 4-molti in racemi: cauli inermi.

R. alpinum Lin. - Sopra Les Ferrières.

Maggio, Giugno.

Ribes alpino it. - Groseiller des alpes fr.

(Calice glabro-racemi maschi a 20-30 fiori, femminei a $2-5$ fiori).

${ }^{*} R$. petraeum Wulf. in JACQ. - Cenisio. (Bertero, Colla Herb. Ped.) (Bonjean in Bertol.).

Maggio, Giugno.

Ribes corallino it. - Gros. des rochers fr.

(Divisioni del calice pressochè sempre cigliate).

R. nigrum Liv. e Off. - Nel Cenisio. Foglie diuretiche e diaforetiche.

Maggio, Giugno. (Calice pubescente glandoloso).

Ribes nero it. - Gros. noir; Cassis fr.

Cenisio (Re in Bertol.). 


\section{R. rubrum Lin. - Ribes Off. - Uva ramà vern.} - Nel Cenisio e sopra Les Ferrières. Frutto rinfrescante.

Maggio, Giugno. (Calice glabro).

Ribes volgare it. - Uva spina, Rasela vern. - Gros. à grappes, Raisin de mars fr.

I Ribes grossularia, nigrum e rubrum sono posti dal Cantani fra i rimedi acidi temperanti.

Il Ribes rubrum, ch'è il preferito dalla coltura, contiene, is preferenza delle altre due specie, acido citrico e malico.

Le foglie e le radici del Ribes nigrum furono adoperate come diuretiche e sudorifere. La farmacia moderna, almeno in Italia, non ne fa più uso.

Ordine XlViI. - Sassifragee: (Saxifrageae Vent.).

Gen. 1. - Saxifraga Lin.

1) Foglie alterne provviste nel margine di pori coperti da una squametta bianca a forma di sevdo. fiante perenni. Fiori bianchi.

* Pori numerosi : foglie linguiformi seghettate.

S. cotiledon LiN. - In tutti i pascoli sassosi montáni ed alpini.

Luglio.

Sassifragia piramidale it. - Saxifrage pyramidal fr. Cenisio (Bonjean in Bertol.).

S. aizoon JACQ. - Frequente alle Gorgie presso Susa e nei siti montani umidi di Giaveno.

Giugno, Luglio.

Sassifragia sedo alpino it. - Sax. aizoon fr. Cenisio (Negri). Musiné, Sagra di S. Michele. Nel testo S. aizoon Lin. dal Murray Syst. 
* Pori 5-7. Foglie lineari oblunghe subtrigone in punta, intere.

S. coesia Lin. - Presso Susa sulla rupe detta delle Tre Pene e siti sassosi del Cenisio.

Luglio, Agosto.

Sassifragia verdemare it. - Sax. bleuâtre fr.

Piccolo Cenisio, rupi sopra Ferrières. (Bonjean in Bertol.).

S. diapensioides BELLARD. - Luoghi sassosi del Cenisio e sopra il Bosco Nero.

Luglio, Agosto.

Sassifragia cinerizia it. - Sax. diapensie fr.

2) Foglie opposte provviste all'apice di $1-3$ pori a squametta eaduca. Fiori rosci o violacei, tal volt a bianchi in $\mathbf{S}$. biflora. Piante perenni.

S. retusa Govan. (S. purpurea Re-ALL.). - Trovata da Ignazio Molineri nelle rupi del Vallon e del Cenisio.

Luglio, Agosto. (Foglie oblungo-trigone).

Sassifragia violetta it. - Sax. écrasée fr.

Cenisio (Balbis, Re, Bonjean in Bertol.) (Bouvier).

Rupi di Monte Pintas e della Punta di Mezzogiorno.

S. oppositifolia LIN. - In tutti i pascoli alpini. Maggio, Luglio. (Foglie ovali ottuse). Sassifragia rosea it. - Sax. a feuilles opposées fr. Cenisio (Bonjean in Bertol.) Assietta.

S. biflora ALL. - Sommità del Cenisio.

Luglio, Agosto. (Foglie obovate o spatolate). Sassifragia rossolina it. - Sax. à deux fleurs fr. Cenisio (Molineri, Balbis e Bonjean in Bertol.). 
B) Foglie lineari alterne provviste all'apice di un tuberculo munito di una fossetta, la citale spesso è glandulosa, mai sưamata. Hiante perenni.

* Petali bianco-giallicci.

S. aspera Lin. - Siti sassosi del Cenisio e siti montani di Chianoc.

Luglio, Agosto.

Sassifragia spinolosa it. - Sax. ciliée fr.

* B. bryoides DC. (Saxifraga bryoides Re-LiN.). Cenisio.

Località medesima (Bouvier) (Negri) (Bonjean in Bertol). - Assietta.

${ }^{*}{ }^{*}$ Petali citrini punteggiati di rancio o tutti aranciati.

S. airoides Lin. (Saxifraga autunnalis RE-LIN.). Comunissima presso i rivoli nei siti sabbiosi della regione montana ed alpina. Scende in pianura, e trovasi qua e là presso Susa lungo la Dora e la Cenisia.

Luglio, Agosto.

Sassifragia autunnale it. - Sax. airoide fr.

4) Foglie sprovriste di pori squamati e di tubereolo. TPiante pereani (ecectto S. Tridactylites che è annua).

* Calice riflcsso: petali bianchi punteggiati di giallo alla base.

S. stellaris Lin. - Presso le fonti alpine. - Cenisio presso il lago.

Luglio, Agosto.

Sassifragia stellata it. - Sax. etoilée fr. 
S. cuneifolia LrN. - Siti ombrosi montani e sopra Giaveno.

Giugno, Luglio.

Sassifragia mestoletta it. - S. ̀̀ feuilles en coin fr.

* * Calice eretto o patente.

1) Petali citrini, raramente rosei o bianchicci

S. muscoides Wulf. (S. caespitosa $\mathrm{R}_{\mathrm{E}-\mathrm{S}} \mathrm{ScOp}$.). - Comunissima nelle rupi delle alpi più alte.

Luglio, Agosto. (Petali citrini raramente rosei).

Sassifragia moseatellina it. - S. mousse fr.

Cenisio (Bonjean in Bertol.) (Negri). Assietta.

Nel testo S. caespitosa Lin. dall'Allioni.

* S. exarata VilL. (S. hiypnoides RE-ALL.).

- Pascoli sassosi alpini.

Giugno, Luglio. (Fiori bianchicci o citrini).

Nel testo S. hypnoides Lin. dal sinonimo di Allioni.

S. sillonnèe fr.

Cenisio (Negri; Colle della Portia.

2) Petali bianchi.

* S. pedemontana ALL. - Cenisio (Bonjean in Bert.).

Giugno, Aǵosto.

Sassifragia ventaglina it. - S. du Piémont fr.

S. planifolia Lapefr. (S. muscoides Re-ALL.). - Siti freddi del Cenisio.

Luglio, Agosto. (Petali disseccati giallicci).

S. verdolina it. $-\mathrm{S}$. à feuilles planes fr.

Nel testo S. muscoides Lin. dal Willd. Spec. 
S. androsacea LiN. - Nei pascoli umidi alpini, specialmente al Cenisio ed all'Assietta.

Giugno, Agosto.

Sassifragia tridentata it. - S. androsace fr.

S. granulata Lin. - Nei pascoli.

Maggio, Giugno.

S. granulata it. - S. granulee fr.

S. bulbifera Lin. - Frequente nei pascoli.

Aprile, Maggio.

S. bulbifera it. - S. bulbifère fr.

S. rołundifolia LiN. - Nei siti montani, ombrosi ed umidetti.

Giugno, Luglio.

S. cimbalaria it. - S. à feuilles rondes fr.

S. tridactylites Lin. - Ovunque nei pascoli aridi. Copiosa presso Susa nei siti sterili della Brunetta.

Ann. Aprile, Maggio, più tardi sulle alpi.

* $\beta$ alpicola Bertol. (S. adscendens JACQ.). - A]tipiano del Cenisio (Negri) - Cenisio (Bonjean in Bertol.).

Erba lattaiola it. - S. à trois doigts fr.

Gen. $2^{\circ}$ - Clhrysosplenium Lin.

C. alternifolium LiN. - Saxifraga aquatica aurea Off. - Frequentissima presso le fonti sopra Giaveno. Farmaco disusato. 
Per. Marzo, Maggio.

Sassifragia dorata it. - Dorine à feuilles alternes fr. Cenisio (Bonjean in Bertol.).

* C. oppositifolium Lin. - Cenisio (Bonjean in Bertol.).

Per. Aprile, Maggio.

Sassifragia citrina it. - D. à feuilles opposées fr.

$$
\begin{gathered}
\text { ORdINE XLVIII. - OMBrelLIFERE } \\
\text { (Umbelliferce Juss.). }
\end{gathered}
$$

Sez. ${ }^{2}$. Fiori verticillati - disposti in eapitoli, in onbrelle semplici, o composte irreggolari.

* Fiori verticillati.

GEN. $1^{0}$ - IIydrocotyle LIN.

H. vulgaris Lin. - Presso le fonti, intorno ai laghi di Avigliana.

Per. Giugno, Agosto.

Soldinella acquatica it. - Hydrocotyle commune, Eccelle d'eau fr.

(Foglie orbicolari peltate).

** Fiori in capitoli.

Gen. 2. - Erymgiam LIN.

E. campestre Lin. o Off. - Pan caud vern. - In tutti i pascoli aridi e sterili. Radice diuretica.

Per. Luglio, Agosto.

Carciofini it. - Panicaut cbampêtre, Chardou Roland fr. Rimedio disusato. 
* * Fiori in ombrelle semplici, o composte irregolari.

Gen. 3. - stumtia Lin.

A. major Lin. - Astrantia o Sanicula foemina Off. - Frequentissima nei prati montani umidetti. Farmaco disusato.

Per. Giugno, Agosto.

Astranzia it. - Astrance à larges feuilles fr.

A. minor Lin. - Cenisio.

Per. Luglio, Agosto.

Astrantia minore it. - Astr. à petites feuilles fr.

Colle della Portiglia.

\section{Gen. 4. - Samieala LiN.}

S. Europaea Lin. - Sanicola Off. - In tutte le selve montane. Risolvente.

Per. Maggio, Giugno.

Sanicola it. - Sanicle fr.

Pianta vantata un giorno come vulneraria e astringente.

\section{Sezione $2^{n}$ - Dmbrelle conaposte regolar.}

A) Perisperma piano o convesso nella faccia commessurale.

* Frutto compresso dai lati (asse maggiore da un dorso all'altro).

Gen. 5. - Bapleuarum Lin.

(Foglie interissime : fiori gialli).

1) Foglie perfogliate ovate.

* B. rotundifolium LrN. - Nei monti intorno Susa (Avogadro in Colla, Herb. Ped., vol. VII, pagina 408). 
Ann. Maggio, Giugno.

Perfogliata it. - Buplèvre à feuilles rondes fr.

2) Foglie non perfogliate.

B. stellatum Lin. - Trovata da Ignazio Molineri nei monti di Giaveno e nella Comba di Chianoc.

Bupleuro stellato it. - Bup. étoilé fr.

Per. Luglio, Agosto.

Bellissima specie.

B. aristatum BartL. (B. odontites Re-Lin.). - Frequente nei pascoli sterili delle Blaccie.

Ann. Giugno, Agosto.

Odontite maggiore it. - Bup. aristé fr.

B. ranunculoides Lin. - Cenisio, Alpi di Giaveno ed anche intorno Susa.

Per. Luglio, Agosto.

Bupleuro scialino it. - Bup. renoncule fr.

Assietta.

B. falcatum Lin. - Presso il Forte diruto di Exilles.

Per. Luglio, Ottobre.

Orecchio di Lepre it. - Bup. en facex fr.

\section{GEN: 6: - Trimia LIN.}

T. vulgaris DC. (Pimpinella dioica RE-Lin. Syst. veg.). - Frequente nei pascoli montani ed alpestri.

Bien. Maggio, Giugno.

Sassifragia pannocchiuta it. - Trinie commune fr. 


\section{Gen. \%. - Aegopodiumbro}

Aeg. podagraria Lin. - Angelica erratica o Podagraria Off. -- Nei siti umidetti e ombrosi. Farmaco disusato.

Per. Giugno.

Podagraria it. - Egopode des goutteux fr.

\section{GEN. 8. - Sison LiN.}

S. amomum LiN. - Amomum vulgare Off. - Nei fossi e nelle siepi lungo la via che conduce da S. Ambrogio ad Avigliana. Gradevolmente aromatica: amaricante carminativa.

Bienn. Luglio, Agosto.

Amomo germanico it. - Sison amome fr.

L'amomo germanico ha le qualita notate dall'autore: non ostante la farmacia d'oggi lo ha disusato.

\section{Gen. 9. - Apiuam Lin.}

A. petroselinum Lis. - Pstroselinum 0ff. - Pnansëmo vern. - Tanto comune in tutti i colli presso Susa e nelle vigne di Meana che io credo possa ritenersi come indigeno.

Bienn. Giugno.

Prezzemolo it. - Persil cultivé fr.

È molto problematica l'opinione dell'autore. Il prezzemolo è spontaneo in latitudini più meridionali e calde; dotato però d'indole robusta e resistente, e da lungo tempo coltivato, non è meraviglia ch' esso sia uscito dal cerchio della coltura in Val di Susa.

Il prezzemolo, come rimedio, tiene ancora il suo posto nella farmacia : è diuretico e fa parte dello sciroppo così detto delle 5 radici aperienti. 
L'infusione dei semi di prezzemolo ed una miscela di olio etereo e grasso ricavato dagli stessi (Apiolo) furono vantate, non sono molti anni, l'una dopo l'altra come mirabile specifico nelle febbri palustri; ma l'entusiasmo duró poco, e non si tardó a riconoscere la superiorita incontestabile della china (Cantani).

* A. graveolens LiN. -- Coltivato.

Bienn. Giugno.

Sedano it. - Seler vern. - Ache odorante fr.

Il sedano è aromatico e leggermente eccitante.

\section{Gen. 10. - Caneurare Lin.}

\section{C. carvi h.s. - Cuminum pratense, Carvi off. -} Orunque nei prati montani ed alpini. Semi carminativi.

Bienn. Maggio, Luglio.

Carvi it. - Carum carvi. - Cumin des près fr.

I semi di questa pianta sono carminativi e digestivi. Si danno in polvere o in infusione nelle dispepsie e nella gastralgie.

C. bulbocastanum KocH. (Bunium bulbocasianum ReLin.). - Bubocastanum off. - Ovunque nei campi montani. Radice commestibile.

Per. Giugno, Agosto.

Bulbocastano it. - Bunium noix de terre fr.

Cenisio alla Gran croce (Bouvier). Sagra di S. Michele.

La radice del bulbocastano sarebbe commestibile, ma non è in uso; è molto ricercata dai maiali.

\section{GEN. 11. - Pimpimerla LIN.}

P. magna Lin. - Copiosa nei prati montani.

Per. Luglio, Agosto.

Tragoselino maggiore it. - Boucage à grandes feuilles fr. 


\section{P. saxifraga Lin. - P. saxifraga, P. alba o Tra-} goselinum Off. - Qua e là nei prati e nei pascoli. Radice stimolante, risolvente.

Per. Agosto, Settembre.

Tragoselino becchino it. - B. saxifrage fr.

Le radici delle due specie di Pimpinella hanno odore non grato e furono adoperate come antispasmodiche e digestive; oggi sono disusate. Le foglie sono ricercate dal bestiame, specialmente dalle pecore.

\section{GEN. 12. - Ptyelhotis Koch.}

P. heterophylla Kocir, (Aethusa Bunius Rr-Murr.-Syst.)

- Ovunque nei monti presso i tonrenti.

Ann. Maggio, Giugno.

Ammi bastardo it. -- Ptycotis is feuilles variées fir.

Via da Susa al Cenisio (Negri).

Nel testo Aethusa Bunius Lin.

\section{Gen. 13, - Siman Lin.}

\section{S. angustifolium Lriv. - Sium o Berula Off. -} Siti acquitrinosi; presso le fonti.

Per. Giugno, Luglio.

Sedanino d'acqua it. - Berle à larges feuilles fr. (Berula angustifolia Koch.).

* Frutto compresso dal dorso (asse maggiore da un lato ad un altro).

\section{Gen. 14. - Laserpicium Lin.}

*. Caule angoloso solcato.

L. prutenicum Lin. - Nei monti di Giaveno. Per. Agosto.

Lasero pimpinellino it. - Laser de Prusse fr. 


\section{Iatifolium Lin. - Gentiana alba Off. - Presso-} chè in tutti i pascoli e prati montuosi ed alpestri. Radice stimolante, stomatica.

Per. Giugno, Agosto.

Erba nocitola it. - L. à larges feuilles fr. Farmaco disusato.

L. hirsutum Lamk (L. Halleri Re-AlL.). - In tutti i prati alpini.

Per. Luglio, Agosto.

Lasero peloso it. - L. velu fr.

Cenisio (Bouvier Negri). Musinè (De-Filippi)

L. siler LiN. - Siler montanum 0ff. - Nei pascoli sassosi montani. Frequente nelle fessure delle rupi presso la Brunetta. Seme aromatico carminativo.

Per. Giugno, Luglio.

Seselio it. - L. siler fr.

Farmaco disusato.

\section{GeN. 15. - Daucus LiN.}

D. carota Lin. - 0. sativus Off. - Abbonda nei prati e nei pascoli. Radice mucilaginosa, emolliente. Seme aromatico.

Bienn. Giugno, Settemore.

Carota selvatica it. - Carotte commune fr.

Non si sa se la Carota comunemente coltivata, dalla quale derivarono parecchie varieta, sia questa specie, o piuttosto il D. maximus Desf., spontaneo in Sardegna.

Anticamente la radice era usata come anticatarrale ed antielmintica. Oggi è disusata. 


\section{Gen. 16. - Drlaya Hoff.}

0. grandiflora Hofr. (Caucalis grandiflora RE-LIN.)

- Frequente nei pascoli aridi e sterili dei colli. Ann. Giugno, Luglio.

Lappola it. - Orlaya à grandes fleurs fr.

GEN. 1\%. - Selinum LIN.

S. carvifolia LrN. - Nei mon乞i di Giaveno.

Per. Giugno, Luglio.

Carvifoglio it. - Selin à feuilles de carci fr.

* Gen. 18. - Tommasina Bertol. Fl. It.

T. verticillaris BentoL. - Sagra di S. Michele (De-Filippi).

Per. Luglio, Agosto.

Gambo di finocchio it. - Tommasinia en verticille fr. (Angelica verticillaris Lin. Mant. All.)

Gen. 18 tis. - maperatonia Lin.

I. Ostrutium Lis. - Imperatoria Off. - Luoghi freddi ed umidi delle alpi. Radice fragrante, aromatica, carminativa.

Per. Giugno, Luglio.

Erba rossa it. - Impératoire commune fr.

Cenisio (Bouvier).

Farmaco ancora in uso.

\section{Gen. 19. - molliea LiN.}

A. sylvestris Lis. e Off. - Presso i ruscelli del Cenisio e presso Susa nelle selve ombrose delle Gorgie. Radice stimolante, aromatica, carminativa. 
Per. Luglio, Agosto.

Angelica selvatica it. - Angélique des forêts fr.

ß. nemorosa Ten. - (Selinum sylvestre Re-AlL.). Presso il Sangone e i ruscelli sopra Giaveno.

Nel testo S. sylvestre Lin. dai sinonimi di Allioni. Auct.

\section{GEN. 20. - Toredlylíum LiN.}

T. maximum Lrv. - Frequente negli spineti, e nei campi.

Ann. Giugno, Luglio.

Ombrellini delle steccic it. - Tordyle majeur fr.

\section{Gen. 21. - Mermelleum Lin.}

H. spondylium Liv. - Spondyium o Branca ursina off. - Comune nei prati umidi e pingui. Radice mucilaginosa dolce, subaromatica.

Per. Luglio, Agosto.

Branca ursina it. - Berce brancursine fr.

* $\beta$ elegans lioch. - Cenisio (Bonjean in Bertol.).

Farmaco disusato.

H. elegans Jacq.

Gen. 22. - Peucedanum Lin.

- Involucro generale nullo, o composto di poche brattee caduche.

P. officinale Lin. - P. o Foeniculum porcinum Off. - Presso Vajes e trovato da Ignazio Molineri sul Musinè. Radice espettorante.

Per. Luglio, Agosto.

Finocchio siciliano it. - Peucédane officinale fr.

Alla Brunetta (Negri). Sagra di S. Michele (De-Filippi). È abbandonato dalla farmacia moderna. 
* Involucro generale a brattee numerose.

P. painstre Moench. (Selinum palustre Re-LiN. Fl. Suec.) - Siti umidi, anche presso il Sangone.

Bienn. Giugno, Agosto.

Selino lattaiolo it. - Selin de marais fr.

(Thysselinum palustre Hoffm. Koch.)

P. alsaticum Lin. - Nei pascoli : frequente nei siti aridi e sterili di Condove alla Roccea.

Per. Agosto, Settembre.

Selino delle selve it. - P. d'Alsace fr.

P. Cervaria Lapey. (Athamanta Cervaria Re-Lin.). Ovunque nei siti montani ai margini delle selve.

Per. Luglio, Agosto.

Cervaria it. - P. des cerfs fr.

P. oreoselinum Moench. (Athamanta Oreoselinum RELiN.). Oreosslinum Off. - Nei pascoli. Leg'germente aromatico.

Per. Luglio, Agosto.

Apio montano it. - P. selin de mortagne fr.

E diuretico (Cantani).

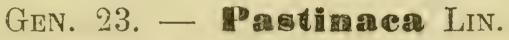

P. sativa LIN. - P. sativa o Ellaphoboscum Off. - Qua e là nei prati pingui e umidetti. Semi aromatici.

Bienn. Luglio, Settembre.

Pastinaca it. - Panais fr.

E diuretica (Cantani). 
*** Frutto sub-cilindrico (assi uguali).

Gen. 24. - Meum Tournf.

M. athamanticum JACQ. (Athamanta Meum RE-LrN.) - Meum Off. - Frequente nel Cenisio. Farmaco disusato.

Per. Maggio, Agosto.

Meo barbato it. - M. athamanthe fr.

M. mutellina GAERTx. (Phellandrium mutellina RE-LiN.) - Ovunque nei prati umidi e freddi.

Per. Luglio, Agosto.

Erba motellina it. - M. mutelline $\mathrm{fl}^{2}$.

Cenisio (Bonjean in Bertol.).

\section{Gen. 25. - Foemieullum Hoffr.}

F. officinale Alt. Ped. (Anethum foeniculum Re-LiN.) - Foeniculum Off. - Fnoi vern. - Frequente nei ruderi. Semi lattiferi, aromatici, risolventi e carminativi.

Per. Luglio, Agosto.

Finocchio selvaggio it. - Fenouil officinale fr.

Secondo il Bertoloni, la specie di Finocchio commestibile e coltivata sarebbe F. dulce DC. annuo non perenne come F. officinale All. Sulla virtù medica del F. officinale il Cantani scrive "Internamente i semi sono un utilissimo carminativo in ispecie per i bambini e giovano assai nella dispepsia, diarrea cronica, ecc. (Mat. med. I, 640). 


\section{Gen. 26. - Aothusa Lin.}

Aethusa Cynapium Lis. - Cicuta minor off. - Frequente nei campi di Condove e specialmente nella regione detta la Grangietta. Velenosa.

Ann. Luglio, Agosto.

Cicuta aglina fr. - Petite ciguë fr.

Questa specie è senza dubbio velenosa e si accosta alla Cicuta virosa. Il suo caule è striato e le toglie 2-3 pinnatipartite. Volgarmente è scambiata per la Cicuta virosa.

\section{Gen. 27. - Gaya Gaud.}

G. simplex Gaud. (Laserpitium simplex Re-Lin.- Mant.) - Alti gioghi del Cenisio.

Per. Luglio, Agosto.

Finocchio d'alpe it. - Gaya simple fr.

Eau blanche al Cenisio (Negri).

Bella specie che si compiace di abitare le ultime vette alpine.

\section{Gen. 28. - Seseli Lin.}

\section{S. annuum LIN. - Pascoli macilenti.}

Bienn. Agosto, Settembre.

Finocchio ferulino it. - Seseli coloré fr.

(S. coloratum Ehrh. Koch.).

Questa specie è data per biennale dal Bertoloni: per annua o perenne dal Koch e da altri autori.

\section{Gen. 29. - Cillomotis Crantz.}

L. montana Alc. (Athamanta Libanotis RE-LiN.) Nei monti di Giaveno, ma rara.

Bienn. Luglio, Agosto.

Libanotide it. - Libanotide de montagne fr. 
154

\section{Gen. 30. - Cmidionu Cuss.}

C. apioides Spreng. (Selimum Seguieri Re-All. Auct.). - Presso il tempio della Sagra di S. Michele: sopra Susa nelle selve del luogo detto La Losa.

Per. Giugno, Agosto.

Piretro vero it. - Cnidie à feuilles de persil fr. Musinè (Re, Fl. Tor.).

Nel testo S. Seguieri Lin. dal sinonimo di Allioni.

\section{GEN. 31. - Trochiseamulhes KoCH.}

T. nodiflorus Koch (Smirnium nodiflorum RE-ALL.). - Nelle selve di Fossimagna e nei prati di Codizar sopra Calmonte.

Per. Giugno, Luglio.

Ligustico it. - Trochisque nodiflore fr.

Sagra di S. Michele (De Filippi).

\section{Gen. 32. - Athomanta FícK.}

A. cretensis Lin. - Daucus creicus 0ff. - Cenisio. Semi acri carminativi.

Per. Giugno, Agosto.

Dauco cretico it. - Athamante de Créte fr.

Farmaco disusato.

\section{Gen. 33. - Demanthe LIN.}

Den. peucedanifolia Poldich - Nei prati e pascoli umidi.

Per. Giugno.

Finocchio napolino it. - Oenanthe à feuilles de peucédan fr.

Nel testo Oen. Peucedanifolia All. 


\section{B) Perisperma concavo}

o concavo introflesso canalato nel margine interno.

* Frutto aculeato pit o meno compresso dai lati.

\section{Gen. 34. - Cauealis Lin.}

C. daucoides LiN. - Volgare nei campi.

Ann. Mäggio, Luglio.

Lappola carota it. - Caucalide à feuilles de carotte fr.

\section{Gen. 35. - Turgegenis Hoffu.}

T. Iatifolia Horfm. (Caucalis latifolia Re-Lrn.). -Il chiaro Allioni afferma che trovasi presso Susa. Ann. Maggio.

Lappola maggiore it. - Turgénie à larges feuilles fr.

Gen. 36. - Torilis Adans.

T. anthriscus Guel. (Tordylium anthriscus Re-LiN.). - Qua e là nelle siepi. Frequente nei campi a Condove.

Bienn. Giugno, Settembre.

Lappola petrosella it. - Torilis des haies fr.

"Frutto lungamente rostrato inerme, pit o meno compresso dai lati.

\section{GeN. 37. - Seeandix LrN.}

S. pecten veneris Lin. - Volgare nelle messi. Ann. Maggio, Giugno.

Spillettoni it. - Scandix peigne de Vénus fr. 


\section{GEN. 38. - mthrigeus HoFfur.}

\section{A. sylvestris HofFm. (Chaerophyllum sylvestre Re-Lin.).}

- Frequentissimo nei prati alpini. Abbonda presso Susa nei prati umidi di San Lazzaro.

Per. Maggio, Luglio.

Cerfoglio selvatico it. - Antrisque sauvage fr.

Cima del Musinè (De-Filippi).

* A. cerefolium Hoffri. - Coltivato. - Cerfoglio, cerfoglietto it. - Cërfojet vern.

Ann. Maggio.

Chaerophyllum sativum Lamk. (Scandix cerefolium Lin.) È indigeno di latitudini nostre più calde.

* Frutto ovato o sublineare, nè rostrato nè aculeato.

Gen. 39. - Conium Lin.

C. maculatum Liv. - Cicuka major Off. - Presso le abitazioni e nelle siepi a Sant'Ambrogio ed Avigliana: non si trova presso Susa. Velenoso e narcotico.

Bienn. Giugno, Luglio.

Cicuta, erba grande it. - Ciguë tachée fr.

Specie velenosissima: da essa e propriamente dai semi fu estratto un'alcaloide (Coniina, Cicutina) micidiale, preso a dose non ordinata, quanto l'Aconitina.

Il grido farmaceutico della cicuta nei tempi presenti $\dot{e}$ scemato, e della pianta si fa più uso esterno che interno.

Bertoloni (Fl. it.) scriveva fin dal 1837 " L'estratto di cicuta si amministra nelle affezioni scrofolose e scirrose, ma credo che la celebrità sia maggiore della virtù. "Nè Cantani opina diversamente. Il caule del C. maculatum è asperso qua e là di macchie purpurce; le foglie sono 3-pinnate. 


\section{GEN. 40. - Chaerophyllum LiN.}

- Petali cigliati.

C. hirsutum Lis. - Ovunque nei prati alpestri. Per. Luglio, Agosto.

Scandice pelosa it. - Cerfeuil hérissé fr.

* C. Villarsii Koch. - Sagra di S. Michele. (Da nota del prof. Arcangeli, Dirett. dell'Ort. bot. Tor.).

Per. Giugno, Luglio.

Scandice it. - C. de Villars fr.

$$
\text { * Petali glabri. }
$$

* C. aureum LAMK. - Cenisio (Negri) (Bonjean in Bertol.).

Per. Giugno, Luglio.

Finocchiella bastarda it. - Cerfeuil doré fr.

Gen. 41. - Molopospermun Foch.

M. cicutarium DC. (Ligusticum peloponesiacum RE-LiN.) - Luoghi elevati di Fossimagna.

Per. Luglio, Agosto.

Seseli peloponese it. - Moloposperme à feuilles de ciguë fr.

Musinè dal lato che guarda Brione. (Re, Fl. Tor.)

Gen. 42. - Plleurospermum Hoff.

P. austriacum Hoff. (Ligusticum austriacum RE-LIN.). - Volgare alla fabbrica del marmo.

Per. Giugno, Agosto.

Cicutaria it. - Pleurosperme d'Autriche fr.

Musinè (Re in Bertol). 


\section{Gen. 43. - Plinsosperom un Cuss.}

Ph. aquiiegifolium Koch. (Ligusticum Lobelii Re.) Alle falde dei monti di Giaveno. Frequente.

Per. Luglio, Agosto.

Ligustico siciliano it. - Physosperme à feuilles d'ancolie fr.

Danaa aquilegifolia All.

Nel testo Ligusticum Lobelii Vill, specie diversa, cioè Cnidium Apioides Spreng. (Vedi pag. 154).

\section{Ordine XliX. - Aragliacee (Araliaceae Jiss.)}

\section{Gen. Unico. - Hedera Lin.}

H. helix Lin. - M. arborsa Off. - Brassabosch vern. - Sul tronco dei vecchi alberi e sulle mura.

Arbusto rampicante. Ottobre.

Edera it. - Lierre grimpant fr.

L'Edera ebbe altra volta il suo posto nella farmacia umana e le si attribuirono parecchie virtù, che oggi sono in gran parte screditate. Ancora nelle campagne si usano le foglie di Edera per tener vivi i cauterii e qualcuno del volgo le usa per curare gli esantemi della testa dei bambini e financo la tigna! 


\section{Ordine L. - Connacee (Comeal DC.)}

\section{Gen. Unico. - Cormus Lin.}

C. sanguinea Lin. - C. foemina Off. - Sanghin vern. - Ai margini dei fossi e delle selve.

Arbusto. Aprile, Giugno (Fiori bianchi).

Sanguinello it. - Cornouiller sanguin fr.

Farmace disusato.

C. mas LiN. - C. sylvestris mas Off. - Cornai vern. - Qua e là nelle selve. Frutto subastringente.

Arbusto. Marzo, Aprile (Fiori gialli).

Corniolo it. - Cornouiller mâle fr.

Male nel testo Cornus mascula Lin.

Come farmaco è disusato. Il frutto (cornai) quand' è maturo è mangiato da talun! con gusto ; il legname è duro e si presta a parecchi lavori.

Ordine Li. - Caprifogliacee (Caprifoliacecle Juls.)

A) Stili 3-5 o stigmi 3 .

Gen. $1^{\circ}$ - Moxa Lin.

A. moschatellina Lin. - Moschatellina o Moschatella Off. - Nei siti ombrosi del Cenisio e sopra il Bosco nero, nè manca nelle alpi di Giaveno è sopra Coazze.

Per. Aprite, Maggio.

Dentaria moscadellina it. - Herbe musquée fr.

Rimedio disusato. 


\section{GEN. $2^{\circ}$ - Sambueras LiN.}

- Caule erbaceo.

S. ebulus Lin. - Ebulus Off. - Nei pascoli e siti incolti. Drastica.

Per. Luglio, Agosto.

Ebolo it. - Petit sureau fr.

Le bacche dell'Ebolo sono leggermente sudorifere; le foglie, la corteccia e la radice servono internamente come un purgante ed esternamente accelerano la cicatrizzazione delle piaghe (Cantani).

\section{* Arbusti o frutici.}

S. nigra Lrv. - Sambucus Off. - Sambù vern. Frequente presso le selve e le case nei luoghi pingrui ed umidetti. Fiori diaforetici risolventi.

Giugno, Luglio.

Sambuco it. - S. noir fr.

Le foglie e le radici del S. nigra sono purgative ed adoperate recentemente con successo nella cura delle idropi asciti; i fiori in infusione riescono diaforetici (Cantani).

S. racemosa Lin. - Alle Ferriere. Aprile, Maggio.

Sambuco montano it. - S. à grappes fr.

\section{GeN. $3^{0}$ - ilburmuan LIN.}

V. Lantana Lin. - In tutte le selve.

Alberetto. Maggio, Giugno. (Foglie ovali).

Lantana it. - Viorne cotonneuse fr.

Cenisio (Bonje an in Bertol.).

V. Opulus Lin. - Nelle selve ed anche lungo i fossi fra Sant'Antonino e la Giaconera.

Alberetto o frutice. Maggio. (Foglie 3-lobate).

Sambuco acquatico it. - V. obier fr. 
B) Stilo filiforme.

(Frutici)

\section{Gen. $4^{\circ}$ - Lonicer a LIN.}

* Cauli scandenti : fiori in fascetti terminali.

\section{L. caprifolium Lin. - Caprifolium italicum Off.} Qua e là nelle selve. Farmaco disusato.

Maggio.

Madreselva it. - Chévrefeuille des jardins fr.

* L. etrusca SANT. - Presso Caselette e nel Musinè (Re, Fl. Tor.). Sagra di San Michele (De-Filippi).

Luglio, Agosto.

Monsorino it. - Ch. d'Etrurie fr.

* Caule eretto rigido: pedunculi biflori.

L. Xylosteum LiN. - Comunissima nelle selve presso Susa.

Maggio, Giugno.

Madreselva pelosa it. - Ch. à bois blanc fr.

L. nigra LiN. - Da me trovata al Cenisio presso la Ramasse.

Maggio, Giugno.

Ciliegia selvatica it. - Ch. à fruits noirs fr. Musinè (De-Filippi).

L. alpigena LiN. - Frequente nelle selve montane di Bussolino e verso la Fabbrica del marmo. Maggio, Giugno.

Cameceraso it. - Ch. des alpes fr.

L. coerulea LIN. - Volgare nelle alpi.

Maggio, Giugno.

Ciliegia alpina cerulea it. - Ch. à fruits bleus fr. 
Ordine LiI. - Rubiacee (Rubiaceae Juss.).

A) Calice a 6 denti visibili, persistenti.

\section{GEN. $1^{\circ}$ - Sherardia Lin.}

\section{Sh. arvensis Liv. - Nei campi.}

Bienn. Giugno, Settembre.

Toccamano it. - Shérarde des champs fr.

B) Denti ed orlo del calice poco o niente visibili.

GEN. $2^{\circ}$ - mperula LiN.

A. arvensis Lis. - Ovunque nei campi.

Ann. Maggio, Giugno. (Fiore ceruleo).

Palloncino it. - Aspérule des champs fr.

A. taurina LiN. - Frequente nelle selve ombrose. Per. Maggio, Giugno. (Fiori bianchi).

Stellina cruciata it. - A. de Turin fr.

A. cynanchica Lin. - Rubia cynanchica off.

Tolgare nei pascoli secchi e sterili. Farmaco disusato.

Per. Giugno, Agosto. (Fiori rosei).

Squinanzia it. - A. à l'esquinancie fr.

Cenisio (Bonjean in Bertol.).

* A. Jordani Perr. e Song. (1). - Cenisio (Bouvier).

Pel. Luglio, Agosto. (Fiori porporini).

Asperule de Jordan fr.

(1) Perrigr e Songeon - Indication de quelques plantes nouvelles: rares, etc. Chambery, 1855. 
A. odorata Lin. - Matrisylvae herba - Asperula odorata o Hepatica stellata Off. - Ovunque nelle selve ombrose. Farmaco disusato.

Per. Maggio, Giugno. (Fiori bianchi). Stellina odorosa it. - A. odorante fr.

\section{Gen. $3^{\circ}$ - Galium Lin.}

1) Foglie trinervate verticillate per quattro.

* Fiori gialli.

* G. pedemontanum AlL. - Su la Brunetta (Negri).

Ann. Maggio, Giugno.

Crociata scabra it. - Gaillet du Piemont fr.

G. vernum Scop. (Valentia glabra RE-Lin.). - Nei pascoli.

Per. Maggio, Giugno.

Crociata minore it. - G. printanier fr.

G. Cruciata Scop. (Valantia Cruciata Re-Lin. - Cruciata Off. - Nelle siepi e nei pascoli. Farmaco disusato.

Per. Maggio.

Crociata it. - C. croisette fr.

* Fiori bianchi.

G. boreale Lin. - Al sito detto le Pertuse in valle d'Exilles e presso il Vallon. Trovasi pure nella valle di Bardonêche e sopra Les Ferrières.

Per. Giugno, Agosto.

Erba croce lanciuolata it. - G. boréal fr. 
2) Foglie uninervate o senza nervi verticillate per 4 o per molte.

* Caule senza aculei : glabro, o pubescente, o irsuto.

G. verum Lin. - G. luteum Off. - In tutti i prati e pascoli. Coagula il latte.

Per. Giugno, Agosto. (Fiori gialli).

Caglio zolfino it. - G. jaune fr.

Fu usato altra volta come antispasmodico e diaforetico come parecchie altre specie di Galium; il Caglio zolfino ha la proprietà di coagulare il latte, dovuta questa all'acido acetico che si sviluppa nella corolla (Cazin).

G. purpureum LiN. - Nei pascoli.

Per. Luglio, Agosto. (Fiori rossi).

Caglio porporino it. - G. pourpre fr.

G. erectum Huds. Bertol. (Galium aristatum Re-Alt. non Lin. sec. Bertol.) - Frequente nel Cenisio, e (G. austriacum Re-All. Auct. non JACQ. sec. Bertor.) - Trovato da Ignazio Molineri nei prati montani di Giaveno, e da me nei prati di Codizar sopra Chiomonte.

$\beta$ lucidum (Galium lucidum RE-ALL.) - Nel monte di Caselette detto il Musinè, sopra Monpantero, e nei siti aridi e sassosi di Foresto.

Per. Maggio, Giugno. (Fiori bianchi).

Caglio punteggiato it. - G. droit fr.

G. Mollugo Lin. - G. album Off. - Comunissimo nelle siepi e nei prati. Farmaco disusato.

Per. Maggio, Giugno. (Fiori bianchi).

Caglio bianco it. - G. blanc fr. 
G. rupicola Bertol. (G. saxatile Re-ALL.) - Luoghi sassosi alpini.

Per. Luglio, Agosto. (Fiori bianchi).

Caglio stellato it. - G. des rochers fr.

Cenisio (Bonjean in Bertol.). Cima di Ronche (Negri sotto G. helveticum Weigel).

Nel testo G. Saxatile Lin. dal sinonimo di Allioni, specie diversa come osserva il Bertoloni Fl. it., vol. II, pag. 109.

G. sylvaticum Lin. - Nelle selve.

Per. Giugno, Agosto. (Fiori bianchi).

Caglio boschereccio it. - G. des bois fr.

G. sylvestre Pollich. « glabrum Косн. (Galium montanum Re-ViLL.). - Raccolto nel Cenisio dal chiar. Bellardi.

Per. Giugno, Luglio. (Fiori bianchi).

Caglio d'Alpe it. - G. sauvage fr.

* G. pusillum Lrn. - Cenisio (Molineri e Bonjean in Bertol.).

B Bocconi (G. Bocconi Re-AlL.) - Luoghi sassosi presso i torrenti alpini. Frequente nel Cenisio.

y megalospermum (G. megalospermum RE-ALL.) Raccolto sul Cenisio dal ch. Allioni.

Cenisio a Ronche (Negri).

o trichophyllum (6. trichophyllum RE-ALL.). - Raccolto da Ignazio Molineri nei monti di Cesana.

Per. Luglio, Agosto. (Fiori bianchi).

Caglio esile it. - G. nain fr. 
* Caule pil o meno aculeato: fiori bianchi.

G. palustre Lis. - Comunissimo nei siti umidi. Per. Maggio, Luglio.

Stellina ruvida it. -- G. des marais fr.

* G. uliginosum Lin. - Trovato presso la Dora nella Valle di Exilles da Felice Valle. (All. Ped., T.1, n. 16) Per. Maggio, Giugno.

Caglio fragile it. - G. fangeux fr.

G. aparine LiN. - Aparine 0ff. - Nei campi ai quali è dannosissimo. Farmaco disusato.

y spurium Kock. (G. spurium Re-LiN.) - Nei pascoli e su le mura.

Ann. Maggio, Agosto.

Asprella: Attaccamane it. - G. gratteron fr.

I semi di questa specie torrefatti furono una volta adoperati a sostituire il caffè (Bertol.).

G. saccharatum AlL. (Valantio aparine RE-LIN.) Aparine Off. - Nei campi. Farmaco disusato.

Ann. Maggio, Giugno.

Erba croce dei grani it. - G. anis sucré fr.

Varie specie di Galium sono polimorfe, e presentano non lieve difficoltà nel determinarle: la loro sinonimia è addirittura un ginepraio, poichè ogni forma fu dai botanici più o meno recenti considerata per caratteristica di una specie.

I Galium in generale se non sono pascoli squisiti per le bestie, non si possono però annoverare tra l'erbe cattive e rifiutate. L'istesso Galium aparine, che il Re chiama infesto ai campi, è mangiato, quando è giovine, con bramosia. 
Ordine LiII. - Valerianacee. (Valerianeae DC.)

\section{Gen. $1^{0}$ - Valariana Lin.}

1) Fiori tutti conformi, ermafroditi.

V. officinalis Lis. - Valerianae radix Off. - Frequente nelle Fratte, nei pascoli, nel Cenisio e nelle selve montane di Chiomonte. Esimio rimedio antispasmodico, antielmintico.

Per. Maggio, Agosto.

Valeriana it. - Valériane officinale fr.

$\grave{\mathrm{E}}$ stato riconosciuto che la V. officinalis val poco o niente come antielmintica e antifebbrile nelle malattie d'infezione. Giova nell'isterismo e contro le neuropatie e contro il meteorismo come carminativo. La radice della V. officinale agisce per il suo olio etereo e per l'acido valerianico, che hanno entrambi la stessa azione fisiologica (Cantani).

2) Fiori difformi, cioè in taluni individui piu grandi a stami sporgenti dalle fauci della corolla, in taluni altri molto minori con stami chiusi nel tubo della stessa e stilo spurgente, in questi le antere sono sterili. (Dioici).

* Foglie cauline-pinnate.

V. disica LiN. - Nei prati umidi subalpini.

Per. Maggio, Giugno.

V. palustre it. - V. dioïque fr.

V. tuberosa Lin. - Siti aprici delle Blaccie e di S. Lazzaro ed a oriente della Brunetta. Copiosissima.

Per. Giugno, Luglio.

Nardoo montano it. - V. tubéreuse fr.

Nella località medesima (Re, Bonjean in Bertol.). 
168

* * Foglie cauline ternate.

V. tripteris Lin. - Siti ombrosi delle alpi.

Per. Giugno, Agosto.

Nardo montano trifogliato it. - V. à trois lobes fr.

** Foglie intere.

V. montana Lis. - Cenisio.

Per. Giugno, Agosto.

Nardo montano fatuo it. - V. de montagne fr.

V. saliunca ALL. - Presso Sestrieres e sopra. Cesana.

Per. Luglio, Agosto.

Nappe rosse it. - V. saliunca fr.

V. celtica Lin. - Sommità del Cenisio verso il piccolo Cenisio. Radice aromatica nervina.

Per. Luglio.

Nardo celtico it. - V. nord celtique fr.

\section{Gen. $2^{\circ}-$ Centharantiluas DC.}

C. angustifolius DC. (Valeriana angustifolia RE-ALL.). - Presso la Dora e nei monti di Cesana: volgare. Per. Maggio, Luglio.

Savonina a foglie strette it. - Centranthe à feuilles étroites fr.

* C. ruber DC. - Naturalizzato nelle roccie del giardino della Sagra di S. Michele.

Per. Giugno, Luglio.

Valeriana rossa it. - C. rouge fr.

Nella stessa località (m. 900 sul mare) insieme al Centhranthus ruber vivono, senza però fruttificare, l'Olivo ed il 
Nespolo del Giappone, piante fuor di dubbio appartenenti a zone assai più meridionali e che non possono attecchire nella pianura torinese, quantunque questa si elevi in media per soli m. 240.

Il curioso fenomeno è spiegato nel seguente modo: allorquando l'atmosfera è tranquilla, come avviene ordinariamente in questa Italia continentale, ed i suoi strati non sono rimescolati dai venti, gl'inferiori diventando più caldi e quindi più leggieri dei superiori salgono, e viceversa i superiori discendono. È l'istesso fenomeno che succede nei teatri: la platea è più fresca dell'ordine superiore dei palchi.

Sappiamo tutti che i siti alquanto più elevati godono in queste condizioni d'una temperatura annua più mite, e sappiamo che l'inverno è meno rigido sulla collina che sulle basse sponde del Po.

\section{Gen. $3^{\circ}$ - Valeriamella Pollich.}

V. olitoria Polt. (Valeriana locusta o olitoria Re-LrN.). - Salzet vern. - Niente di più volgare nei campi. Si mangia in insalata.

Ann. Maggio, Giugno.

Dolce mangiare it. - Mâche potagère fr.

V.coronata DC. (Valeriana locusta $y$ coronata RE-LiN.). - Nelle vigne presso Susa.

$\star$ nn. Aprile, Maggio.

Valeriana canuta it. - M. couronnée fr. Alle Blaccie ed a Gravere (Negri) (V. hamata Bast.) 
Ordine LIV. - Dipsacee (Dipsaceae DC.)

\section{Gen. $1^{0}$ - Dipsacus LiN.}

D. sylvestris Lin. - D. sylvestris o Labium veneris Off. - Pento d'Masche vern. - Dovunque nei siti incolti lungo i fossi. Farmaco disusato.

Bienn. Luglio, Settembre.

Scardiccione it. - Cardère sauvage $\mathrm{fr}$.

D. Iaciniatus Lin. - Siti umidi presso i prati.

Bienn. Luglio, Settembre.

Pettine it. - C. decoupée fr.

D. pilosus Lin. - Siti ombrosi.

Bienn. Luglio, Agosto.

Verga di pastore it. - C. velue fr.

Gen. $2^{\circ}$ - Cephralara Schrad.

C. transylvanica Schrad. (Scabiosa transylvanica RELiN.). - Qua e là nei campi presso la Novalesa. Ann. Giugno, Agosto.

Vedovina maggiore it. - Scabieuse de Transylvanie fr.

Gen. $3^{\circ}$ - Succisa Mertens e Koch.

S. pratensis Moench. (Scabiosa Succisa Re-Lin.). Scabiosa o Morsus diaboli Off. - Abita i prati umıdi alpini e montani. Frequente nelle alpi di Mochie, e presso i laghi di Avigliana.

Per. Luglio, Agosto.

Morso del diavolo it. - Scabieuse succise fr.

Le foglie furono reputate toniche, sudorifere e buone con- 
tro le malattie della pelle dagli antichi medici. Oggi sono pressochè disusate in farmacia. È detta Morsus diaboli perchè la radice è tronca alla sua estremità come per effetto di un morso; la fantasia dei nostri buoni antenati attribui questo morso al diavolo.

\section{Gen. $4^{\circ}$ - Fíngatia Coult.}

K. arvensis Coult. (Scabiosa arvensis Re-Lin.) Scabiosa Herba Off. - Scabiosa vern. - Nei prati secchi. Amaricante.

Per. Luglio, Agosto.

Vedovina selvatica it. - Scabieuse des champs fr.

(Trichera arvensis Schrad.).

Rimedio pressochè disusato: aveva, secondo gli antichi, virtù simili a quelle possedute dalla Succisa pratensis.

K. sylvatica Dub. bot. gall. (Scabiosa sylvatica RELiN.). - Nelle selve montane ed alpestri.

Per. Maggio, Giugno.

Vedovina ruvida it. - Scabieuse des bois fr.

(Trichera sylvatica Schrad.).

\section{Gen. $5^{0}$ - Seabiosa Lin.}

S. columbaria LIN. - Presso i campi e i pascoli secchi e nelle rupi aride di Condove sopra la Grangietta.

Bienn. o Per. Giugno, Agosto.

Vedovina selvatica it. - Scabieuse colombaire fr.

* S. lucida Vill. - Sponda del lago del Cenisio (Bonjean in Bertol.). Cenisio (Bouvier).

Per. Giugno, Agosto.

Scabieuse luisante fr. (S. Columbaria $\chi$ Bertol.) 
S. pyrenaica ALL. - Pascoli sassosi, declivi presso Susa e la Novalesa. Frequente sopra Foresto e nella contrada di Fossimagna.

Bienn. o Per. Giugno, Agosto.

Vedovina bianca it. - S. des Pyrénées fr.

\section{Ordine LV - Composte (Compositae Lin.)}

Sez. ${ }^{2}$ - Capitoli a fori quasi sempre tutti tubolosi ; fiori della circonferenza talvolta più o meno disformi dad centrali ma giammai ligulati e veramente raggianti come, p. es., mel Rellis perennis (

A) Foglie mai spinose:

squame dellinvolucro sprovviste di appendici e di aculei.

Gen. $1^{\circ}$ - Eupatonium LIN.

Eup. cannabinum Lin. - Eup. cannabinum o Trifolium cervinum Off. - Comunissimo vicino ai fossi e nei luogi umidi. Radice diuretica, catartica, emetica.

Per. Giugno, Agosto. (Fiori purpureo-pallidi).

Eupatorio it. - Eupatoire à feuilles de chanvre fr.

L'Eupatorio merita più considerazione di quella in cui ̀̀ tenuto dai medici moderni. Avvenne per esso un discredito esagerato dopo un credito alla sua volta esagerato.

Le radici e il caule dell'Eupatorio possono servire da utile purgante nelle stitichezze ostinate, e come idragogo nelle idropisie e nelle stasi della vena porta; serve anche bene da espettorante (Cantani). 
Gen. $2^{\circ}$ - Allomostylles Cass.

(Fiori purpurei).

- Fiori 3-6 per capitolo.

A. albifrons Renb. (Cacalia albifrons Re-LiN.) - Raccolta da Ignazio Molineri al Cenisio al sito detto Comba di Ambin.

Per. Luglio, Agosto.

Cacalia petasite it. - Cacalie velue fr.

A. alpina Bluff. e Fing. (Cacalia alpina Re-Lin.) Siti umidi e ombrosi delle Alpi.

Per. Luglio, Agosto.

Cacalia maggiore it. - C. des alpes fr.

${ }^{* *}$ Fiori 12-20.

* A. leucophylla Rehb. - Cenisio all'Eau blanche (Huguen. in Bouvier).

Cenisio (Re, Append. alt., pag. 203, sotto il nome di Cacalia hybrida). Cenisio, (Bonjean in Bertol.)

Per. Luglio, Agosto.

C. cotonnosa it. - C. à feuilles blanches fr.

\section{Gen. $3^{\circ}$ - Petasites Gaertn.}

*Fiori rosei, o roseo-pallidi.

P. officinalis Moench. (Tussilago Petasites Re-Lin.) Petasites Off. - Piuttosto comune nei prati acquosi di montagna. Espettorante.

Per. Marzo, Aprile.

Petasite it. - Pétasite officinal fr.

Farmaco disusato. 
- Fiori bianchi o roseo-pallidi.

P. albus Gaertn. (Tuss:lago alba Re-Lin.) - Frequente nei siti ombrosi di montagna e presso $i$ torrenti.

Per. Aprile, Maggio.

Farferugio bianco it. - P. blanchâtre fr.

* P. niveus Cass. - Cenisio (Bonjean in Bertol.) Per. Maggio.

Farferuglio velutino it. - P. blanc de neige fr.

Gen. $4^{\circ}$ - Womogymo CASSIN.

H. alpina CASS. (Tussilago alpina RE-LIN.) - In. tutti i pascoli alpini.

Per. Giugno, Agosto. (Fiori bianchi, o porporini).

Tussilagine liscia it. - Homogyne des alpes fr.

\section{GEN. $5^{0}$ - Limosyris DC.}

(Fiori giallo-dorati)

L. vulgaris Cassin. (Chrysocoma linosyris RE-LIN.) Valle di Cesana, sul monte di Caselette (Musinè) e sul monastero della Novalesa.

Per. Luglio, Agosto.

Spilli d'oro it. - Crysocome à feuille de lin fr.

\section{Gen. $6^{\circ}$ - Mieropus LIN.}

M. erectus Lin. - Comunissimo sopra Foresto e Fossimagna.

Ann. Giugno, Luglio. (Fiori gialli).

Bambagia lanosa it. - Micrope dressé fr. 


\section{Gen. $7^{\circ}$ - IBidens LiN.}

B. tripartita LiN. - B. nostras Off. - Ovunque presso i fossi. Farmaco disusato.

Ann. Luglio, Settembre. (Fiori gialli).

Canapa acquatica it. - Bidens tripartite fr.

B. cernua Lin. (Coreopsis bidens RE-LiN.) - Siti umidi presso la Dora.

Ann. Luglio, Settembre. (Fiori gialli).

Forbicina intera it. - B. penchè fr.

Nella Bidens cernua non di rado i fiori della circonferenza sono ligulati neutri (B. Cernua $\beta$ Koch): nella $B$. tripartita assai raramente (B. tripartita $\beta$ Willd.): ma il genere è chiaramente distinguibıle dagli acheni senza pappo armati per lo più di due aculei.

Gen. $8^{\circ}$ - andolina LiN.

S. chamae-cyparissus Lin. -- Abrotanum foemina, Santolina chamae-cyparissus Off. - Nelle rupi della Brunetta. Tonica, carminatıva, antielmintica.

Per. Luglio, Agosto. (Fiori gialli).

Crespolina it. - Santoline cyprés fr.

La Santolina è appena oggi adoperata in farmacia come stomatica, antispasmodica ed altielmintica.

\section{Gen. $9^{\circ}$ - Tanacerum Lin.}

\section{T. vulgare Lin. - T. Off. - Dovunque lungo} i fossi, nelle selve, piuttosto raro intorno Susa. Amaro, stomatico.

Per. Luglio, Agosto. (Fiori gialli).

Tanaceto it. - Tanaisie commune fr.

Questa specie, secondo il Cantani, si usa principalmente con successo in terapeutica come antielmintica contro gli ascaridi, gli ossiuri e le tenie. Essa agisce per l'olio etereo 
che contiene, il quale è cosi potente che preso in dosi non ordinate produce avvelenamento.

Glı antichi credettero a moltissime virtù del Tanaceto ‘ oggi discreditate.

GeN. 10. - Filago Lin.

F. germanica LIN. - Filago o herba impia Off. Nei pascoli sterili.

Ann. Luglio, Agosto. (Fiori bianco-giallicci).

Bambagia selvatica it. - Cotonnière d'Allemagne fr.

Farmaco disusato.

F. arvensis Lin. - Pascoli sterili.

Ann. Luglio, Agosto.

Canapicchia piramidale it. - C. des champs fr.

\section{GeN. 11. - Gmaploalium LIN.}

" Fiori della circonferenza a piu serie femminei: del disco ermafroditi: pappo filiforme o appena ingrussato all'apice : fiori gialli.

G. sylvaticum LIN. - Comune nei siti ombrosi montani ed alpini. Copioso nelle alpi di Mocchie, nella regione detta di Barmoscel.

Per. Luglio, Agosto.

Leontopodio falso it. - Gnaphale des bois fr.

* G. norvegicum Gunner. Fl. Norv. - Cenisio (Bouvier).

Per. Luglio, Agnsto.

G. de Norwége fr.

G. supinum LIN. - Siti freddi del Cenisio.

Per. Luglio, Agosto.

Canapichia sottile it. - G. couché fr: 


\section{G. luteo-album LiN. - Presso le mura.}

Ann. Luglio, Agosto.

Canapicchia pagliata it. - G. jaunâtre fr.

G. uliginosum Lıs. - Luoghi umidi già inondati. Ann. Luglio, Settembre.

Canapicchia palustre it. - G. des marais fr.

* Fiori della circonferenza a pith serie femminei a pappo sub-filiformo del disco ermafroditi a pappo ingrossato (claviforme) all'apice.

G. leontopodium Scop. (Filago leontopodium RE-LiN.). Abbonda nei pascoli alpini.

Per. Luglio, Agosto. (Fiori giallo-verdi).

Leontopodio vero it. - Pied-de-lion des alpes fr.

È il famoso Edelweiss della Svizzera.

* Capitoli dioici : gli ermafroditi sterili : pappo ingrossato all'apice: fiori bianchi o bianco-rosei.

G. dioicum Lin. - Hispidula o Pes cati Off. Dovunque nei siti aprici, montani e alpini. Fiori espettoranti.

Per. Maggio, Giugno. (Fiori bianco-rosei).

Bambagia selvatica it. - G. dioïque - Pied de chat fr.

Farmaco disusato.

G. carpathicum Wahlenb (G. alpinum Re-Willd。 Bertol.). - Sommità del Cenisio.

Per. Luglio, Agosto.

Canapicchia livida it. - G. des Carpathes fr.

Nel testo: G. alpinum Lin. dall'Allioni.

G. Carpathicum (Re, App. III ad Fl. ped., 1829, pag. 14).

G. margaritaceum Lin.-Visto da Pietro Cornaglia nel Cenisio, come scrive il ch. Allioni. 
Allioni (Fl. Ped., I, pag. 171) dà per indigena questa specie di Gnaphalium, ed accenna ad un'altra località presso Torino (Vanchiglia) ove l'avrebbe rinvenuta il Bellardi. Ma, fuor di dubbio, cadde in errore. Linneo (Spec. plant., ed. III, vol. II, pag. 1198) la dichiara originaria d'America, nè diversamente opina Alfonso Decaudolle Geog. bot., vol. II, pag. 723) su dati incontestabili. Il Gnaphalium margaritaceum fu introdotto in Europa dopo la scoperta di America e fu coltivato nei giardini inglesi, e qua e là sul continente. Non farebbe meraviglia quindi che il Bellardi l'abbia trovato fuggitivo da qualche giardino nei prati di Vanchiglia, ma sul Cenisio?

\section{GEN. 12. - Artentaia LIN.}

(Acheni senza pappo)

* Ricettaculo peloso.

\section{A. absinthium LiN. - Absinthum 0ff. - Incens} vern. - Frequente nei siti alpestri: comunissima alla Brunetta presso il Forte diruto di Exilles. Stomatica, antielmintica.

Per. Luglio, Agosto. (Fiori citrini).

Assenzio it. - Armoise absinthe fr.

Agisce in modiche dosi per il suo principio amaro (Absintina come farmaco stomatico: peró l'abuso dell'assenzio, specialmente sotto forma di liquore alcoolico, è capace di produrre gravi perturbamenti nervusi e danneggiare principalmente il cervello. (Cantani, Nothangel).

A. mutellina Vir.L. (A. rupestris Re-All.). - Genepi album Off. - Moncenisio. Diaforetica, amara.

Per. Luglio, Agosto. (Fiori citrini).

Genipi bianco it. - A. mutelline, Génipi blanc fr.

Cenisio a Ronche (Negri).

Nel testo A. rupestris Lin. dall'Allioni. 
A. glacialis Lrv. - Absinthium alpinum o Genepi nigrum Off. - Moncenisio e nelle sommità dell'Assietta. Virtù della precedente.

Per. Luglio, Agosto. (Fiori giallo-dorati).

Genipi nero it. - Armoise des glacier's fr.

Cenisio a Ronche (Bonjean in Bertol.).

* "Ricettacolo glabro: fiori gialli.

* A. tanacetifolia All. - Nel monte detto il gran Parè presso Sestrières (All. Ped.).

Per. Luglio, Agosto.

Assenzio tanacetiuo it. - A. noirâtre fr.

A. campestris Lin. - Abrotanum campesire 0 ff. - Ovunque nei pascoli e nei campi secchi. Amara, stomatica.

Per. Luglio, Agosto.

Amarella it. - A. champêtre fr.

A. spicata JAcQ. (A. Boccone Re-Alt.). - Cenisio: valle d'Oulx e sopra Giaglione. Emula le virtù dell'A. glacialis.

Per. Luglio, Agosto.

Genipi a spica it. - A. en épi fr.

Cenisio a Ronche (Negri), Rocciamelone (Huguen. in Bouvier).

\section{A. vulgaris Lin. - Artemisiae herba Off. - Qua}

e là nei siti incolti. Amaricante, stimolante.

Per. Luglio, Settembre.

Abrotano dei cam i it. - Armoise, Couronne de St. Jean fr. Antielmintica e diaforetica (Cantani). Fu stimata anche per lo passato come antisterica.

Le specie antecedenti di Artemisia hanno più o meno proprietà stomatiche ed amare. L'A. glacialis, mutellina e spicata eutrano nel liquore detto Genepi des alpes. 


\section{Gev. 13. - Saussurea DC.}

(Fiori purpurei: pappo interno piumoso, esterno semplice)

S. alpina DC. (Serraiula alpina RE-LiN.). - Presso il lago del Cenisio.

Per. Luglio, Agosto.

Sarretta lanuginosa it. - Saussurée des alpes fr.

Sponde meridionali del lago del Cenisio (Bouvier).

* S. depressa Grenier. - Cenisio (Huguen. in Bouvier).

Per. Luglio, Agosto.

Sarretta depressa it. - Saussurée deprimée fr.

\section{GEN. 14. - BBerardia VILL.}

(Pappo semplice, contorto a spirale alla base)

B. subacaulis VirL. (Onopordon rotundifolium Re-ALL.). - Nei monti di Cesana.

Per. Luglio, Agosto. (Fiori bianchicci).

Cardo sgambato it. - Bérardie fr.

GeN. 15. - Serratula Lin.

Fiori purpurei raramente bianchi : pappo semplice.

S. tinctoria Lin. - Serratula 0ff. - Presso le selve nei lnoghi montani. Ha proprietà tintoria eccellente.

Per. Luglio, Agosto.

Serratula it. - Sarriette des teinturiers fr.

Creduta un giorno vulneraria, oggi disusata.

La Serratula infusa dá alle lane trattate con l'allume un color giallo durevole ed un bel verde di Sassonia se le stesse sono trattate col solfato d'indigo. Ad essa però è preferita dai tintori la Reseda luteola Lin. 


\section{Gen. 16. - Crupina Pers.}

(Fiori porporini: pappo semplice)

G. vulgaris Pers. (Centaurea crupina Re-Lin.).

Qua e là nelle vigne e nei pascoli aridi presso Susa e specialmente di Monpantero: nè manca nelle vig'ne di Exilles.

Ann. Maggio, Giugno.

Centaurea crupina it. - Centaurée crupine fr.

B) Foglie mai spinose: squame dell'involucro uncinate all'apice, o provviste di spine o di appendici, raramente nude:

\section{GEN. 1\%. - Lapa TournF.}

(Fiori porporini: pappo semplice)

L. major Gaertx. (Arctium Lappa Re-Lin.). - Bardana Off. e vern. e dalla grente di Matthie detta Napolia - Nelle siepi e nei ruderi. La radice è diuretica.

Bienn. Luglio, Agosto. (Squame dell involucro uncinate). Bardana it. - Bardane à grosses têtes fr.

La radice di Bardana in decozione è reputata anche presentemente diuretica, e leggermente diaforetica da usarsi nella gotta e nel reumatismo cronico. Fu anche ad essa. ma senza fondamento, attribuita la virtù di far ricrescere i capelli.

\section{Gen. 18. - Leuzera DC.}

(Pappo piumoso)

L. conifera DC. (Centaurea conifera RE-Lin.). Nella valle sopra Foresto dal lato che guarda Susa.

Per. Maggio, Agosto.

Centaurea strobilina it. - Leuzée conifère fr. (Fiori rossi: appendici scariose ovato-lacere). 


\section{Gen. 19. - Cemtaurea Lin.}

1) Squame dell'involucro nide.

C. alpina Lis. (Centaurea centaurium RE-BaLbis) Centaurium maius o Centaurea maior Off. - Abbonda nei monti sopra Foresto. Amara.

Per. Luglio, Agosto. (Fiori gialli).

Centaurio giallo it. - Centaurée des alpes fr.

Il Bertoloni (Fl. it., vol. 9, pag. 428) dimostra con evidenza che i botanici piemontesi, tra i quali Balbis e Re, ritennero per C. Centaurium Lin. la C. Alpina dell' istesso autore. Infatti sotto il nome di C. Centaurium il Balbis mandò al Bertoloni esemplari della C. Alpina a fiore giallo come li possiede questa specie, mentre che la C. Centaurium li ha rossi e vegeta nel mezzogiorno d'Italia sulla Majella, sul Volture e nel Leccese.

2) Squame dell'involucro terminate da 3-5 spine.

C. sols'itialis LrN. - Spina solstitialis Off. - Nei pascoli e nei campi. Amara.

Ann. Luglio, Settembre. (Fiori citrino-gialli).

Cardo stellato, Erba della Miadonna it. - C. du solstice fr.

3) Squame dell'involurro terminate d'appendici munite tutte o parte, o prive di frangie e di ciglia.

- Fiori porporini : raramente bianchi

a) Acheni senza pappo.

C. nigrescens Willd. (C. nigra Re-All. non LiN. sec. Bertol. Fl. it., V. 9, pag. 434). - Jacea nigra o Jacea vulgaris Off. - Dovunque nei prati. Diuretica.

Per. Giugno, Agosto.

Fioraliso grande it. - C. noire fr.

Bouton da preive, Bouton d'j'ebreo vern. 
C. jacea Lin. - Jacea Off. - Nei prati della Novajesa e nel Cenisio. Diuretica.

Per. Maggio, Giugno.

Jacea maggiore it. - C. jacée fr.

C. amara Lin. - Volgare nei pascoli aridi.

Per Luglio, Settembre.

Steccioni it. - C. amère fr.

b) Acheni papposi.

C. Rhapontica Lrv. - Sopra Cels nella valle d'Exilles.

Per. Giugno, Luglio.

Centaurea rapontica it. - C. Rhapontique fr.

C. uniflora Lis. - Frequente nei prati e nei pascoli alpini.

Per. Luglio, Agosto.

C. bianca it. - C. à une fleur fr.

* C. nervosa Willd. - Musinè (Re, Fl. Tor. sotto il nome di C. phrygia).

Per. Luglio, Agosto.

C. zazzerina it. - C. plumeuse fr.

C. paniculata Lin. - Comunissima nei pascoli. Bienn. Maggio, Agosto.

C. pannucchiata it. - C. en panicule fr.

C. scabiosa Lin. - Abbonda nei campi e nei prati montani ed alpini.

Per. Luglio, Agosto.

C. vedovina it. - C. scabieuse fr. 
- Fiori ceruleo-violacei : acheni papposi.

C. Cyanus Lin. - Cyanus Off. - Volgarissima nelle messi. Dà un colore ceruleo.

Ann. Maggio, Giugno.

Fiordaliso it. - C. bleuet fr.

* C. axillaris Willd. - Musinè (Re, Fl. Tor.).

$y$ Carniolica Косн. (C. Triumphetti Re-Alz.). - Cenisio e presso il Forte di Fenestrelle.

Per. Luglio, Agosto.

Ciano maggiore it. - C. axillaire fr.

C. montana Liv. - Comune nelle selve e nei prati montani.

Per. Luglio, Agosto.

Centaurea montagnola it. - C. des montagnes fr.

Le specie di Centaurea notate dal Re come medicinali non sono più in uso nella farmacia moderna.

\section{Gen. 20. - Xearatheman LiN.}

(Pappo fatto di paglie lanceolate).

Kerant. cilindraceum Siвтн e Sir. (Keranthemum annuum RE-Alr.). - Siti sassosi aprici della Brunetta, fra Oulx e Cotaplana.

Ann. Luglio, Agosto. (Fiori porporini).

Perpetuini piccoli it. - Immortelle fr.

(Squame superiori dell'involucro terminate da linguette roseo-purpuree raggianti).

Nel testo Xerant. annuum Lin. dall'Allioni. 
C) Foglie più o meno spinose nei margini, talvolta anche al di sopra: squame dell'involucro parte o tutte aculeate (acuminate lesiniformi all' apice e cigliate nel genere Echinops.)

\section{Gen. 21. - Eehinops Lin.}

(Pappo brevissimo coroniforme).

E. sphaerocephalus Lin. - Presso la Dora ed a Condove nella regione della Grangietta pressu il torrente dei Cervot.

Per. Luglio, Settembre. (Fiori bianchi).

Echinopo it. - Echinope à tête ronde fr.

E. ritro Lin. - Presso Susa e Borgone nel luogo detto Pietra cullèra.

Per. Luglio, Settembre. (Fiori cerulei).

Echinopo minore it. - E. ritro fr.

Alle Blaccie ed a Gravere (Negri).

\section{Gen. 22. - Carlina Lin.}

(Pappo piumoso).

C. acaulis LrN. - Carlinae radix Off. - Ciardousse vern. - Nei luohi montani ed alpini. Comunissima. Rimedio disusato.

Per. Luglio, Agosto.

Carlina it. - Carline sans tige fr.

C. vulgaris Lrn. - Frequente presso le selve submontane o montane.

Bienn. Luglio, Agosto.

Carlina comune it. - C. commune fr. 
C. acanth:folia ALL. - Qua e là nella valle di Exilles presso Cesana e sopra Venaus, nè manca intorno Susa nei siti sassosi non lontano dalla Chiesetta della Madonna della Coà ed in siti aprici selvatici ad oriente della Madonna della Losa.

Bienn. Giugno, Agosto.

C. zolfina it. - C. à feuilles d'acanthe fr.

\section{Gen. 23. - Dmopordom Lin.}

(Pappo semplice).

0. Acanthium Lin. - Onopordon o Acanthium Off. - Ama i ruderi, ed i siti sassosi specialmente presso le ab.tazioni. Il succo recente è commendato esternamente contro il cancro.

Bienn. Luglio, Agosto. (Fiori porporini).

Acanzio it. - Onoporde acanthin fr.

E addirittura insussistente che il cancro possa guarire col succo dell Onopordon.

I Cardellini mangiano avidamente i semi di questa pianta.

Gen. 23. - Cirsium Tournf.

(Pappo piumoso)

a) Capitoli ermafroditi...

1) Foglie spinolose al dissopra: fiori purpurei.

C. lanceolatum Scop. (Carduus lanceolatus Re-Lin.) - Dovunque nei pascoli e nei siti incolti.

Bienn. Giugno, Agosto. (Foglie decorrenti).

Cardo it. - Cirse lanceolè fr. 


\section{G. eriophorum Scop. (Carduus Eriophorus Re-Lin.).} - Comunissimo nei pascoli e nei siti montani incolti.

Bienn. Luglio, Agosto. (Foglie non decorrenti).

Cardo scardaccio it. - C. laineuz fr.

\section{2) Foglie inermi al di sopra.}

* Foglie decorrenti o sub-decorrenti : flori porporini.

G. monspessulanum Azt. (Carduus monspessulanus Rk-Lin.) - Nasce nei prati irrigati ad Lixilles e nella Valle di Cesana e di Bardonêche.

Per. Giugno, Luglio.

Cardo ciglioso it. - C. de Montpellier fr.

C. palustre Scop. (Carduus palustris Re-Lin.) Presso i fossati dei prati umidi e presso le fonti nei siti paludosi.

Bienn. Luglio, Agrosto.

Cardo di palude it. - C. des marais fr.

* * Foglie non decorrenti.

C. erisithales Scop. (Cnicus Erisith?les Re-Lin.). Qua e là nei prati macilenti e presso i campi montani.

Per. Luglio. (Fiori gialli).

Cardo montano it. - C. glutineux fr.

\section{C. spinosissimum Scop. (Cnicus spinosissimus RE-LiN.)}

\section{- Cenisio.}

Per. Luglio, Agosto. (Fiori gialli).

Cardo capitato it. - C. très-épineux fr. 
2) Capitoli per lo pit solitari, raramente gemini o terni.

C. nutans LiN. - Ovunque nei pascoli montani ed alpestri.

Ann. Giugno, Luglio. (Fiori porporini odorosi).

Cardo rosso it. - Ch. penchè fr.

* C. spinolosus Bertol. - Cenisio (Bonjean e Balbis in Bertol.).

Ann. Luglio. (Fiori porporini).

Cardo spinosetto it.

* C. defloratus Lin. - Presso il Lago del Ce. nisio (Negri).

Per. Giugno, Luglio. (Fiori purpurei).

Cardo dentellato it. - Ch. terne fr.

\section{Gen. 27. - Kentroployllum Neck.}

(Pappo fatto di pagliuzze)

Kent. lanatum DC. (Carthamus lanatus RE-LIN.). Atractyls Marth. - Volgrare nei siti aridi e sterili: a Conduve nella Contrada detta la Roccea.

Ann. Luglio, Settembre. (Fiori gialli).

Scardiccione it. - Kentrophylle laineux fr. 
Sez. $2^{2}$ - Capitoli formati guasi sempre da fiori del tatto disformi : quelii del dises tabulosi : quelli della periferia naldbiadi (a liaguetta) arachianti.

A) Fiori del disco e linguette del medesimo colore.

1) Acheni sormontati da un pappo semplice: fiori gialli.

GEN. 28. - LIN.

- Capitoli vestiti alla base di piccule squame (Caliculo).

S. vulgaris Liv. - Senecio Off. - Ovunque negli orti e nei campi. Farmaco disusato.

Ann. Marzo, Settembre.

Senecione it. - Seneçon commun fr.

Questa è la sola specie di Senecio fra le nostre alla quale quasi sempre mancano le linguette periferiche: peró l'involucro inerme rinforzato alla base da $8-10$ piccole squame nere all'apice, e le foglie inermi la distinguono dalle specie contenute rella sezione $L^{2}$.

S. nemorensis LrN. ( S. Saracenicus RE-All. non LiN.). - S. Saracenicus Off. - Nelle selve ombrose montane. Vuluerario, astringente.

Per. Luglio, Agosto. (4-5 linguette).

Senecio selvano it. $-\mathrm{S}$. des sarrasins fr.

Nel testo S. Saracenicus Lin. dall'Allioni.

Farnaco disusato.

S. viscosus Liv. -- Pascoli montani.

Ann. Luylio, Agosto. (Linguette brevissime poco apparenti, rivolte).

Senecione viscido it. - S. visqueux fr.

S. Jacobaea Lrs. - Jacobaea Off. - Prati e pascoli umidi. Farmaco disusato.

Per. Giugno, Agrosto.

Senecio maggiore it. - Herbe-de-Jacob fr. 
S. Doronicum Lin. - Nei prati e nei pascoli montani ed alpini.

Per. Maggio, Agosto.

Senecio mezzano it. - S. Doronic fr.

Cenisio all'Eau Blanche, e presso il lago (Verlot).

S. incanus Lin. - Sommità del Cenisio.

Per. Luglio, Agosto.

Senecio ombrellato it. - S. blanchâtre fr.

Cenisio (Bonjean in Bertol.). Assietta.

S. uniflorus AlL. - Raccolto da Ignazio Molineri nelle sommità delle Alpi di Giaveno al Colle della Rossa.

Per. Luglio, Agosto.

Senecio semplice it. - S. uniflore fr.

Cenisio (Bonjean in Bertol.).

* S. erraticus Bertol. Rar. it. pl. - Presso il lago di Caselette (Giusta in Colla Herb., vol. III, pag. 403).

Per. Luglio, Agosto.

Astero dei fossi it. $-\mathrm{S}$. à rameaux grêles $\mathrm{fr}$.

* Capitoli nudi alla base.

S. aurantiacus DC. (Cineraria alpina RE-JACQ-ALL. - Alpi di Giaveno. Cenisio.

Per. Giugno, Luglio.

Cineraria Crocata it. - S. orangé fr.

Nel testo Cineraria alpina Lin. dall'Allioni. 
Gen. 28. - Tussillago Lin.

\section{Tussilago farfara Lis. - Farfara o Tussilaginis} folia, flores 0ff. - Farfara vern. - Comune alle sponde dei fiumi e dei torrenti. Espettorante.

Per. Marzo, Aprile. (Foglie orbicolari angolose).

Farfara it. - Tussilage pas-d'âne fr.

La Farfara in infusione si adopera con qualche vantaggio nelle diarree croniche. La sua proprietà espettorante è molto problematica (Cantani). Inutile nella tisi (Nothangel).

GeN. 29. - Inula Lin.

(Pappo tutto conforme)

1) Squame interne dell'involucro dilatate all'apice; spatulate.

I. helenium Lrs. - Enula campana, Inula o Helenium Off. - La trovai nel Cimitero di Bussolino. Radice stimolante, espettorante, stomatica, antielmintica.

Per. Luglio, Agosto.

Elenio it. - Inule aunée, Oeil-de-cheval fr.

Di tutte le virtù dell'Elenio segnate dal Re rimane oggi ben poco. Esso non fa più parte dei farmaci moderni.

2) Squame interne dell'involucro acuminate all'apice. * Acheni glabri.

I. hirta LiN. - Nelle selve.

Per. Maggio, Giugno.

Enula scabra it. - I. hérissée fr.

Musinè.

I. salicina Liv. - Qua e là nei prati magri. Presso Susa nei siti collinosi delle Blaccie e tra la Trinità e S. Marciano.

Per. Luglio, Agosto.

Spragginella it. - I. à feuilles de saule fr. 
I. squarrosa Lis. - Nelle selve presso Susa alle falde dei monti.

Per. Luglio, Agosto.

Enula uncinata it. - I. coriace fr.

** Acheni irsuti o pubescenti.

I. conyza DC. (Conyza squarrosa Re-Lin.) - Conyza major Off. - Frequente nei pascoli aprici intorno alle selve. Presso Susa nei siti sassosi alle falde del Colle di S. Lazzaro. Aromatica, stimolante.

Bien. Luglio, Agosto.

Coniza it.' - I. conyze fr.

I. montana Lis. - Comunissima nei siti montani presso Susa, Monpantero, Foresto e Fossimagna, ecc.

Per. Giugno, Agosto.

Enula canuta it. - I. de montagne fr.

Gen. 30. - Pulliearia Gaertn.

(Pappo esterno coroniforme denticolato, o crenato)

P. vulgaris Gaertx. (Inula Pulicaria Re-Lin.). - Conyza minor 0ff. - Nei pascoli umidi disseccati in estate. Rimedio disusato.

Ann Luglio, Agosto.

Enula pulciaia it. - Pulicaire commune fr.

P. dysenterica Gaertn. (Inula dysenterica Re-Lin.). Conyza media Off. - Copiosa nei siti umidi presso i fossi. Farmaco disusato.

Per. Luglio, Agosto.

Menta selvatica it. - P. dyssentérique, Herbe de SaintRoch fr. 


\section{Gen. 31. - Solidago Lin.}

S. virga aurea Lin. - Virgaurea 0ff. - Frequente nelle selve montane. Amaricante, diuretica e vulneraria.

Per. Luglio, Settembre.

Erba giudaica it. - Herbe des Juifs fr.

Musinè.

Fu raccomandata come dotata di potente azione sui reni, ma è oggi ben poco usata (Cantani), anche per le indicazioni segnate dal Re.

S. minuta Lis. - Cenisio.

Per. Luglio, Agosto.

Verga d'oro semplice it. - Solidage verge-d'or fr.

\section{Gen. 32. - Doronicam Lin.}

D. pardalianches Lin. e Off. - Abbonda nelle selve montane. Radice lievemente aromatica.

Per. Giugno, Luglio. (Acheni periferici senza pappo).

Doronico it. - Doronic mort aux panthères fr.

Rimedio disusato.

\section{Gen. 33. - Mromicum Necker.}

A. Clusii Косн. Deutsch. (Arnica Clusii Re-All.) Alpi di Giaveno.

Per. Luglio, Agosto. (Acheni tutti papposi). Arnica doronico it. - Aronic doronic fr.

A. scorpioides Kocr. (Arnica scorpioides RE-Lrn.).Abbonda nel Cenisio: e nelle Alpi di Giaveno.

Per. Luglio, Agosto.

Arnica scorpioide it. - A. scorpion fr. 


\section{GEN. 34. - Arnica LIN.}

\section{A. montana Lis. - Arnica Off. - Tabac d' mon-} tagna vern. - Frequente nei prati alpini. Acre, stimolante, errina, diuretica, emenagoga.

Per. Giugno, Luglio.

Arnica it. - Arnica de montagne fr.

Musinè (Re, Fl. Tor.) Sagra di San Michele.

Dell'Arnica si fece grande elogio e grande uso dalla vecchia clinica: come rimedio interno fu adoperato nel tifo, nelle pneumoniti asteniche, nelle convulsioni epilettiformi, nelle paralisi, nella dissenteria, ecc., e giunse a meritare da Stall, come rimedio antimalarico, il titolo di china del povero: ma oggi, come farmaco interno, è caduto quasi nell'oblio, e non del tutto meritamente, dappoichè potrebbe per lo meno tentarsi internamente in varie neuropatie, essendo certo che l'Arnica è un eccitante del cervello e del sistema nervoso in generale. Come rimedio esterno il suo grido si è mantenuto nella farmacia moderna, e giova fuor di dubbio in casi in cui occorre facilitare il riassorbimento degli stravasi sottocutanei avvenuti per cause traumatiche ed attivare il processo di nutrizione e di risoluzione in altri casi, dipendenti da cause organiche, ulceri, tumori, ecc.

Le radici dell'Arnica contengono acido tannico e riescono più astringenti dei fiori. Questi sono ricchi invece di un principio amaro e di un olio etereo (arnicina) e spiegano una maggiore azione sul sistema nervoso (Cantani): polverizzati, sono fortemente ervini.

2) Acheni privi di pappo o a pappo searioso coroniforme.

\section{GEN. 35. - Buplothalmum LiN.}

Buph. salicifolium Lis. (Buphthalmum grandiflorum Re-All. non Lin. sec. Bertol.) - Presso i torrenti nei 
siti montani ed alpini. Frequente nel Cenisio. Presso Susa ad oriente del Borgo di San Marciano.

Per. Giugno, Luglio (Linguette numerose lineari: fiori gialli; pappo coroniforme).

Asteroide salicina it. - Buphthalme à feuilles de saule fr.

\section{GeN. 36. - Achillea LIN.}

* Fiori gialli : per lo pitu 5 linguette metà pith brevi dell'involucro: foglie pinnatifide.

Ach. tomentosa Lin. - Ovunque nei pascoli sterili e specialmente verso la Brunetta e sopra Foresto.

Per. Maggio, Giugno.

Millefoglio giallo it. - Achillée cotonneuse fr.

- * Fiori bianchi : per lo pit 6-10 linguette lunghe quanto l'involucro: foglie pinnate, o pinnatifide, o dentate.

Ach. macrophylla LiN. - Frequente nei siti ombrosi delle alpi.

Per. Luglio.

Achillea matricalina it. -.- A. à grandes feuilles fr.

Altipiano del Cenisio (Negri).

Ach. herba-rota AlL. - Herba rotta Math. - Fra Giaveno e Fenestrelle nel sito detto Malanotte. Amara. sudorifera.

Per. Luglio, Agosto.

Erba rotta it. - A. herba rota fr.

Nel testo è scritto Herba rotta: nell'Allioni, Herbarota. Allioni (Ped., vol. I, pag. 181) magnifica le virtù medicinali dell'Erba rotta. Secondo lui è diaforetica, emenagoga, antielmintica, carminativa, antimalarica. Non ostante ciò la farmacia moderna ne fa poco anzi nessun uso. L' abbandono è giustificato? Certo gli alpigiani l'hanno in gran conto e per essi fu ed è ancora una panacea. 
* Ach. moschata Wurf. - Cenisio (Billet in Colla Herb., Vol. VII, pag. 415).

Per. Luglio, Agosto.

Erba livia it.

Ach. nana Lin. - Herba rotta Off. - Erba d'la rotta vern. - Sommità del Cenisio e nelle alpi di Giaveno. Amara, aromatica, stomatica.

Per. Luglio, Agosto.

Achillea villosa it. - A. naine fr.

Altipiano del Cenisio (Negri).

Stimata per le virtù notate dal Re dagli alpigiani : poco o niente usata nella farmacia cittadina moderna.

** Fiori bianchi o rosei : foglie bipinnatifide.

Ach. millefolium Lin. - Millefolium 0ff. - Millefeui vern. - Dovunque. Amara, leggermente stimolante, stomatica.

Per. Maggio, Agrosto.

Achillea: Millefoglio it. -- A. millefeuille fr.

Il Millefoglio ha un'azione press'a poco simile a quella della salvia, favorendo la digestione ed eccitando leggermente il sistema nervoso (Cantani).

A. tanacetifolia Willd. Bertol. (A. magna Re-Alt. non Lrs. sec. Bertol.). - Alpi di Giaveno: Cenisio.

Per. Luglio, Agosto.

Achillea tanacetina it. - A. à feuilles de tanaisie fr.

Musinè (Re, Fl. Tor.).

Nel testo A. Magna Lin.

A. nobilis LIN. - Millefolium nobile Off. - Campi montani, subaromatica.

Per. Luglio, Agosto.

Achillea siderite it. - A. noble fr.

Presso Susa (Molineri in Bertol.) 
$B \backslash$ Fiori del disco quasi sempre a colore dissimile da quello delle linguette.

"Acheni sormontati da un pappo sernplice : fiori del disco gialli o giallicci :

linguette bianchiccie, cerulee, lilacine, purpuree. o bianche.

\section{Gen 37. - Eaigeron LiN.}

E. canadense Lin. - Comunissimo nei campi.

Ann. Luglio, Agosto. (Linguette bianchiccie).

Impia it. - Vergerette de Canadà fr.

Questa brutta specie (Erba j)anà; Pnas d'volp vern.) che infesta i campi, e sale finanche sui tetti delle case, venne a noi col commercio dall'America del Nord. Nel 1655 era pianta coltivata nel giardino di Blois col nome di Aster canadensis annuus: da quell'epoca man mano si propagó in Europa dalla Sicilia alla Svezia!

E. acre Lrn. - Nei pascoli: presso Susa nei siti sassosi della Brunetta.

Bien. o Per. Luglio, Agosto. (Linguette lilacine anguste incospicue).

Asterano it. - Verg. âcre fr.

\section{E. alpinum Lis. - Cenisio.}

Per. Luglio, Agosto. (Linguette purpuree).

Ceppica alpina it. - Verg. des Alpes fr.

Assietta.

\section{E. uniflorum Lin. - Sopra Les Ferrières e nel} Cenisio.

Per. Luglio, Agosto.

Cenisio (Bouvier senza indicazione più precisa di località).

La specie trovata dal Re alle Ferrières ed al Cenisio e da lui indicata come E. uniflorum Lin. è probabilmente una forma deil' E. alpinum Lin. Ciò confessa l'istesso Re (App. III ad Fl. Ped., pag. 16) creando sulla sola diversità 
di colore del pappo, un suo E. uniflorum appunto per distinguerlo dal Linneano ritenuto da lui non come specie, ma come semplice varietà dell'Alpinum. Tutto ciò è anche confermato dagli esemplari raccolti sul Cenisio ed esistenti nell'Erbario dell' Ort. bot. di Torino. (Vedi anche Bertoloni, Fl. it., Vol. IX, pag. 102).

* E. Villarsii Bellard (E. purpureus Colla Herb. Re, App. III). - Presso Salbertrand (Re, App. III) Cenisio (Huguen. in Bouvier).

Per. Luglio, Agosto. (Linguette purpuree).

Ceppita scabra it. - E. de Villars fr.

\section{GEN. 38. - Aster LiN.}

A. alpinus Lin. - Abbonda nel Cenisio, alpi di Gravere e nelle alpi di Giaveno.

Per. Luglio, Agosto. (Linguette cerulee).

Astero alpino it. - Aster des alpes fr.

A. amellus LrN. - Abbonda a Fossimagna, presso Susa nelle vigne a mezzodi della Brunetta e nei siti sassosi di San Costanzo. Lo trovai pure nei pascoli aprici di Condove nel luogo detto la Rocea.

Per. Luglio, Agosto. (Linguette violaceo-cerulee). Amello it. - A. amellus fr.

\section{Gen. 39. - Bellidiastnum CASS.}

B. Michelii CASS. (Doronicum bellidiastrum RE-LIN.). - Frequente nei siti montani ed alpini subumidi.

Per Luglio, Agosto. (Linguette bianche, o purpuree al disotto).

Margherita d'alpe it. - Pâqueronie de Michéli fr.

Blaccie e Gravere (Negri). Sagra di s. Michele. 
* Gen. 40. - Stentatis Cassin.

St. annua NeEs - Nelle siepi umidette e presso i fossi, specialmente lungo la Dora.

Ann. Maggio, Agosto. (Linguette bianche).

Astraceli margheritino it. - Sténactis annuelle fr.

* Acheni senza pappo:

fiori del disco gialli, linguette bianche, o rosee al disotto.

\section{Gen. 41. - Bellis Lin.}

B. perennis LrN. - Bellis minor 0ff. - Margaritin vern. - Comune dovunque. Rimedio disusato.

Per. Luglio, Agosto.

Margheritina it. - Pâquerette vivace, Margherite fr.

\section{GeN. 42. - nthemis LiN.}

A. cota Lin. - Nei campi montani.

Ann. Maggio, Giugno.

Cota it. - Cota élevée fr.

A. arvensis Lin. - Nei campi.

Ann. Maggio, Agosto.

Camomilla bastarda it. - Anthémides des camps fr.

A. cotula Lin. - Cotula foetida Off. - Presso le abitazioni e le aje. Antispasmodica.

Ann. Luglio, Agosto.

Camomilla mezzana it. - Camomille des chiens fr. Farmaco disusato.

GEN. 43. - Matricaria Lin.

M. Chamomilla Lis. - Chamoemelum vulgare o Chamomilla Off. - Camamia vern. - Abbonda nei 
campi e nei siti aridi presso le vie. Fiori fragranti, amari, lievemente stimolanti, antispasmodici.

Ann. Maggio, Luglio.

Camomilla it. - Camomille fr.

I fiori di questa pianta sono utilissimi come digestivi e carminativi; s'usano pure come diaforetici e come eccitanti generali del sistema nervoso (Cantani). Farmaco notissimo.

M. inodora Lis. suec. ed. II. (Chrysanthemum inodorum Re-LiN.). - Nei campi.

Ann. Luglio, Agosto.

Matricale selvatica it. - Faussa camamia vern. - Matricaire inodore $\mathrm{fr}$.

(Tripleurospermum inodorum C. H. Schult\%).

\section{Gen. 44. - Lenearathemam Tournf.}

L. vulgare DC. Prod. (Chrysanthemum leucanthemum RE-LiN.). - Bellis major: Leucanthemum vulgare: Oculus bovis Off. - Subacre, diuretica. In tutti i prati e nei pascoli.

Per. Maggio, Agosto.

Bellide maggiore it. - Margarita dii prà vern. - Grande marguerite fr.

Tenuto per vulnerario e diuretico : oggi disusato.

* L. montanum DC. Prod. (Chrysanthemuin heterophyllum Balbis. Re, Fl. Tor. - Chrysanthemum montanum Lin. - Chrisanthemum leucanthemum $\beta$ Bertol.). - Abtıonda sul Musinè (Re, Fl. Tor.)

Per. Maggio, Agosto.

Bellide montanum it. - Leucanthème de montagne fr. 
I. atratum DC. Prod. (Chrysanthemum atratum ReLin.). - Da me trovato presso la borgata di Mochie detta li Cinati.

Per. Giugno, Luglio.

Occhio di bue grandissimo it. - L. noir fr.

\section{Gen. 45. - Pyredlarum Sm.}

P. alpinum Willd. (Chrysanthemum alpinum RE-Lin.). - Frequente nelle Alpi.

Per. Luglio, Agosto.

Matricale alpino it. - Pyrethrum des alpes fr.

P. corymbosum Willd. (Chrysanthemum corymbosum RE-LiN.). - Intorno alle selve.

Per. Giugno, Agosto.

Cotagrande it. - Pyr. en corymbe fr.

P. parthenium Willd. (Matricaria parthenium REJin.) - Matricaria parthenium 0ff. - Da me raccolta in San Michele della Chiusa.

Partenio it. - Pyr. matricaire fr.

Pianta stimata anche presentemente come stimolante, e adoperata specialmente nell'amenorrea e come antisterica, $e$ in generale in tutti i casi nei quali si adopera la Matricaria camomilla. 


\section{Sez. $B^{2}$ - Capitoli formati di fori tutti a lingwette.}

A) Acheni senza pappo.

GEN. 46. - Lapgama LIN.

(Fiori gialli).

L. communis LiN. - Lapsana Off. - Volgare presso i campi e negli orti. Rimedio disusato.

Ann. Maggio, Luglio.

Cavolo selvatico it. - Herbe aux mamelles fr.

\section{Gen. 47. - Cichorian LIN.}

(Fiori cerulei).

C. intybus Lin. - Cichorium Off. -- Sicoria vern. - Volgarissima nei pascoli. Amara, febbrifuga.

Per. Luglio, Settembre.

Cicoria selvatica it. - Chicorée sauvage fr.

La radice di Cicoria più che amaro-tonica è leggermente purgativa, ed è messa dal Cantani tra i rimedi amari salini risolventi. Torrefatta e polverizzata serve ad imposturare la bevanda del caffè: e l'impostura oggi è giunta a tal punto che i semi della famosa pianta arabica non ci entrano soventi che di nome.

* C. endivia Lin. - Coltivata negli orti.

Bienn. Luglio, Agosto.

Endivia it. - Andivia bianca vern. - C. endive fr.

Si crede originaria delle Indie orientali. 
B) Acheni, almeno quelli del disco, con papps piumoso. Gen. 48. - Thrincia Ro'tH.

(Fiori gialli).

T. hirta Roтн. (Leontodon hirtum Re-Lin.). - Raccolta da Felice Valle nel sito detto Traversière nella Valle di Exilles: io la raccolsi presso Susa.

Per. Maggio, Agosto. (Pappo negli acheni del margine breve).

Trinciatella prataiuola it. - Thrincie hérissée fr.

\section{Gen. 49. - Leontodom Lin.}

(Fiori gialli).

- Rizoma tronco-trasverso.

L. taraxaci Lors. (Hieracium taraxaci Re-LrN.). Nella Valle d'Oulx: Cenisio e specialmente a Ronche.

Per. Luglio, Agosto. (Pappo niveo).

Radichiella pisciacane it. - Liondent de montagne fr.

Cenisio (Bonjean in Bertol.) Cenisio a Ronche Bouvier).

Ad una gentile pianticella che abita i culmini alpini e rallegra le loro solitudini coi colori dorati delle sue corolle, Bertoloni diede il brutto e prosaico nome di Radichiella pisciacane ! È proprio il caso di ribattezzarla; la chiamerei Liondente dorato d'alpe.

L. pyrenaicum Gouan. - Frequente nei pascoli del Cenisio.

Per. Luglio, Agosto. (Pappo bianco-sporco).

Pisciacane squamelloso it. - Liondent des Pyrénées fr.

Ecco un altro nome italiano indecente dato dal Bertoloni a questa specie pur essa alpina; perchè non potrebbe chiamarsi Liondente alpino?

I. proteiforme Vill. (L. hastile RE-LiN.). - Nei prati di Fossimagna. 
a vulgaris Косн. (L. hispidum Re-LiN.). - Piuttosto comune nei prati e nei pascoli.

$\beta$ glabratus Koсн. (L. danubiale Re-JACQ.). - Nei siti umidi.

Per. Giugno, Agosto.

Radichiella it. - L. en fer de lance fr.

L. autumnale Lis. - Ovunque nei campi. Luglio, Ottobre.

Radıcchiella tardiva it. - L. d'automne fr.

- Radice verticale discendente.

* L. crispum Vılt. Sul Musinè (Re, Fl. Tor. sotto Apargia crispa) ed a Cesana (Balbis, Misc. bot. alter. sotto lo stesso nome).

Per. Giugno, Agosto.

Radicchiella it. - L. crèpu fr.

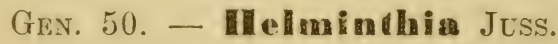

(Fiori gialli).

H. echioides Gaertn. (Pieris echioides Re Lin.). - La raccolsi nei pascoli sterili ed aridi di Condove alla Roceea.

Ann. Giugno, Agosto.

Aspraggine it. - Helminthie viperine fr.

Notiamo che il compendio della Fl. it. Cesati, ecc., non riporta come piemontese questa specie.

\section{GeN. 51. - Pieris Lin.}

(Fiori gialli).

P. hieracioides Lin. - Località medesime dell'Helminthia echioides.

Ann. Giugno, Agosto.

Lattaiuola pungente it. - Picride épervière $\mathrm{fr}$. 
GEN. 52. - Tragopogom LIN.

- Fiori violacei.

T. porrifolium Lis. -- Lo trovai nei siti aprici delle Blaccie. Commestibile.

Bienn. Giugno, Luglio.

Barba di becco it. - Salsifis à fenilles de poircau fi.

Notiamo che questa specie non è riportata come piemontese dal compendio della Fl. it. Cesati, ecc.

\section{T. crocifolium Lin. - Presso Susa.}

Bienn. Maggio, Giugno.

La Flora innanzi citata non fa cenno della localitì Segusina: la riporta delle vicinanze d'Ala in Piemonte.

Sassefrica gramignole it. -- S. à feuilles de safran fr.

* Fiori gialli.

T. pratense Lin. - Tragopogon o barba hirci Off. Barbaboc vern. - Ovurue nei prati. Commestibile.

Bienn. Maggio, Giugno.

Barba di becco it. - Barbe de bouc: Ratabout salsifis des prés fr.

Il nome vernacolo in Colla (Herb.) e Barbabouc non Barbaboc come troviamo nell'Allioni e nel Re. Pianta assai nota: le foglie sono mangiate con piacere per lo piu in salsa acida. La radice fu annoverata in tempi antichi tra $i$ rimedi cosi detti pettorali : oggi è farmaco pienamente dimenticato. 
208

\section{Gen. 53. - Seogrzonera Lin.}

(Fiori gialli).

S. austriaca WiLLD. ( S. humilis RE-JAcQ. non LIN.) - Abbonda nei monti sopra Giaveno, e nei siti montani di Fossimagua. La radice è amaricante.

Per. Maggio, Giugno.

Scorzonera barbuta it. - Scorzonère d'Autriche fr.

Musinè (Re, Fl. Tor. anche col nome di S. humilis Lin.). Malamente nel testo $\mathrm{S}$. humilis Lin.

* S. tenuifolia Schrad. - Nei prati di Balmaforte sopra Bussolino. (Balbis col nome di S. Austriaca, Misc. bot. alt., pag. 24).

Per. Giugno, Luglio.

Scorzonera alpina it. - S. des alpes fr.

* S. hispanica Lin. - Nei prati sopra Sestrières (All. auct. pag. 14).

Per. Giugno, Luglio.

Scorzonera di Spagna it. - S. d'Espagne fr.

\section{Gen. 54. - Podospermum DC.}

(Fiori gialli).

P. laciniatum DC. Fl. Fr. (Scorzonera laciniata RELrv.). - Nelle vie dal Monte Mejer ai quattro denti in Valle di Exilles.

Bienn. Aprile, Maggio.

Scorzonera sbrandellata it. -- Podosperme découpé fr.

P. decumbens Gren. e Godr. (Scorzonera resedifolia Re-Retz-Bellard non Lin.) - Raccolto dal chiaro Bellardi in Val di Susa.

Bienn. Aprile, Maggio.

Scorzonera sbrandellata it. - P. chausse-trape fr. 
Scorzonera resedifolia Lin. i il Sonchus chondrilloide: Desf. o Zollikoferia chondrilloides DC. pianta siciliana e spagnuola. L'errore del Bellardi che diede la nostra specie per Sc. resedifolia Lin. (App. ad Fl. Ped., pag. 35) fu ripctuto dal Re.

\section{GEx. 5.). - - Hypoehoerio Lin.}

(Fiori gialli)。

- Peli del pappo tutti piumosi.

H. maculata Lis. - Frequente nei prati e nei pascoli montani.

Per. Giugno, Agosto.

Porcellina macchiata it. - Porcelle tachée fr.

Varia a foglie non macchiate.

H. uniflora Lix. - Alpi di Giareno andando verso il Colle della Russa.

Per. Luglio, Agosto.

Porcellina ispida it. - P. uniflore fr.

Cenisio (Balbis in Bertol.).

* Peli esterni del pappo pochi e non piumosi.

H. radicata LiN. - Ovunque nei pascoli e specialmente nei siti aprici e sterili di Condove e alla Roccea.

Per. Maggio, Agosto.

Porcellina giuncolina it. - P. enracinée fl. 


\section{C) Acheni con pappo semplice. \\ GEx. 56. - ClHomdrolla LIN.}

(Fiori gialli : pappo pedicillato).

C. juncea Lrs. e Off. - Ovunque nei siti arenosi e sassosi soleggiati. Farmaco disusato.

Bienn. Luglio, Agosto.

Condrilla lattaiola it. - Chondrille effilée fr.

\section{(7Ev. 57. - Taraxacum Juss.}

(Fiori gialli : pappo pedicillato).

T. vulgare LAmk. (Leontodon Taraxacum RE-LiN.) Dens Leonis o Taraxacum 0ff. - Ovunque nei prati e nei pascoli. Amaro.

Per. Marzo, Settembre.

Tarassaco: Dente di leone it. - Sicoria salvaia, virasoi vern. - Pissenlit fr.

Il Tarassaco è proteiforme e si adatta a località assai disparate. Vegeta in Sicilia al livello del mare (T. obovatum DC.) e a grandi altitudini su tutta la catena delle Alpi. (T. vulgare $y$ alpinum Kock).

Come farmaco il Tarassaco occupa il primo posto tra i cosi detti amari salini, cioè amari leggermente purgativi, e la moderna terapia se ne avvale.

\section{GeN. 58. - Prenamthes LrN.}

(Fiori purpurei : pappo sessile).

P. purpurea Lin. - Selve ombrose montane ed alpestri.

Musinè (Re, Fl. Tor.). Seive della Chiusa di San Michele. 
$\beta$ angustifolia Kock. (P. tenuifolia Re-LiN.). - Rinvenuta da Ignazio Molineri nei monti di Giaveno, e da me nelle alpi di Chianoc, e sopra Bussolino.

Per. Giugno, Agosto.

Lattuga montana it. - Prénanthe pourprée fr.

Monti di Bussolino (Balbis in Bertol.). Musinè (Re, Fl. Tor.)

\section{Gen. 59. - Mulgedium CASS.}

(Fiori cerulei : pappo sessile).

M. alpinum Less. (Sonchus alpinus RE-LiN.). Selve del Cenisio.

Per. Luglio, Agosto.

Cicerbita azzurra it. - Mulgédie des alpes fr.

Gen. 60. - Zaeymtha Tournf.

(Fiori gialli : pappo sessile : acheni esterni gobbi a pappo laterale).

Z. verrucosa GaERTN. (Lapsana zacyntha Re-LiN.). - Frequente nei pascoli sterili di Condove alla Roccea.

Ann. Maggio, Giugno.

Radicchio verrucoso it. - Zacynthe à verrues fr.

Gen. 61. - Laetuea Lin.

(Pappo pedicillato).

1) Fiori azzurri.

L. perennis LiN. - Frequentissima nelle rupi e intorno alle vigne di Susa, di Monpantero e di Foresto, ecc.

Per. Aprile, Giugno.

Lattuga montana it. - Laitue vivace fr. 
I. tenerrima Pourr. (L. segusiana RE-BALBIS). Nelle rupi e nei siti elevati sopra le vigne di Monpantero, e di Foresto.

Per. Luglio, Agosto.

Lattuga radicchiella it. - L. délicate fr.

2) Fiori gialli.

- Capitoli di sei o pith fiori.

L. saligna Lis. -- Nei campi.

Bienn. Luglio, Agosto.

Lattuga saligna it. - L. à feuilles de saule fr.

L. scariola Lin. - Predilige i ruderi e i siti sassosi. Acre, diuretica, sospetta.

Bienn. Luglio, Agosto.

Scariola it. - L. sauvage fr.

(Lactuca augustana All.).

L. virosa Lin. - Siti incolti. Virtù della precedente.

Bienn. Luglio, Agosto.

Lattuga velenosa it. - $\mathrm{L}$. vireuse $\mathrm{fr}$.

Presso il tempio della Sagra di San Michele (De Filipui).

Le foglie e il succo di queste due specie di lattuga riescono sedative, anodine e ipnotiche, e tengono un posto nclla farmacia moderna tra $\mathrm{i}$ narcotici. Hanno però azione pii debole degli oppiati.

La lattuga coltivata, Lactuca sativa Lin., e le sue numerose varietà, discendono dalla Lactuca scariola (Alf. I)ecandolle, Koch, Moris). La Lattuga coltivata ha le proprieti medesime ma piu deboli delle specie selvaggie. 
* Capitoli di cinque fivri.

L. muralis Fresen. (Prenanthes muralis Re-LiN.). Frequente su le mura e nelle selve ombrose umidette.

Ann. Luglio, Agosto.

Crespigna dolce it. - L. des murs fr.

L. viminea Link. (Prenanthes viminea RE-LiN.). Siti sassosi di Monpantero e di Foresto: presso la Cenisia a Susa.

Per. Luglio, Agosto.

Crespigno alato it. - L. osier fr.

\section{Gen. 62. - Somehus Lin.}

(Fiori gialli : pappo sessile).

S. arvensis Lin. - Siti umidi presso le acque. Per. Luglio, Agosto.

Crespino dei campi it. - Laitron des champs fr.

S. oleraceus Liv. - Sonchus Off. - Negli orti e nei siti pingui. Rimedio disusato.

Ann. Maggio, Agosto.

Cicerbita liscia it. - L. des lieux cultivés fr.

Gen. 63. - Crepis Lin.

1) Pappo sessile: fiori gialli o giallo-dorati.

* Caule pith o meno foglioso.

C. montana DC. (Hieracium pontanum Re-Murray. Srst.). - Frequente nei prati pingui del Cenisio. Per. Giugno, Luglio.

Geracio porcellina it. - Soyerie de montagne fr.

Nel testo Hieracium pontanum Lin. 
C. pigmaea LiN. - Nei monti di Cesana ma rara.

Per. Luglio, Agosto.

Geracio brunellino it. - Crepide naine fr.

Nasce in luoghi aridissimi e tra i macigni frantumati nella Valle Etroite al sito detto Le Mollinet....; nei monti di Cotaplana sopra Oulx, e sopra Cesana. (All. ped. $1^{\circ} 215$ ). Hieracium prunellaefolium Gouan. Willd. All.

C. blattarioides VilL. (Hieracium austriacum REHofrm. Deut. Flor.). - Nel Cenisio.

Per. Luglio, Agosto.

Geracio a saetta it. -- Crepide fausse blattaire fr.

Nel testo Hieracium austriacum Lin.

G. grandiflora DC. (Hieracium grandif́lorum RE-ALL.). - Alpi di Chianoc: Cenisio.

Per. Luglio, Agosto.

Geracio astato it. - Cr. à grandes fleurs fr.

Altipiano del Cenisio (Negri).

G. paludosa Moench. (Hieracium paludosum Re-Lin.). - La raccolsi nei monti di Codizar supra Chiomonte: è frequente nel Cenisio, siti umidi presso il lago e i rivoli.

Per. Luglio, Agosto.

Geracio liscio it. - Cr. des marais fr.

Isolotto del lago del Cenisio (Negri).

C. polymorpha Waur. (Crepis virens RE-Vill.). Nei campi.

Ann. Giugno, Settembre.

Radicchiella prataiola it. -- $\mathrm{Cr}$. verdâtre $\mathrm{fr}$.

Nel testo: Crepis virens Lin, dal Willd. sp. 
* C. pulchra Lin. - Io la trovai presso la Dor'a nell'agro Torinese: D. Valle presso Exilles. (All. Ped., vol. 1, pag. 120).

Ann. Maggio, Giugno.

Erba dolce it. - $\mathrm{Cr}$. élégante $\mathrm{fr}$.

Prenanthes hieracifolia (Re, Fl. Tor.)

* Cauli scapiformi, nudi, o muniti di una o due foglie.

c. aurea Cass. (Leontodon aureum Re-LiN.). - Nei pascoli umidetti e freddi delle alpi: a me occorse specialmente nelle alpi di Monpantero, e del Cenisio.

Per. Luglio, Agosto.

Radicchiella ranciata it.

Presso la cima dell'Assietta dal lato che guarda Argueil.

2) Acheni muniti di un rostro che figura come il pedicillo del pappo.

(Barkhausia DC.)

- Rostro degli acheni esterni piu brevl.

C. foetida Lin. - Frequente presso Susa, Monpantero, ecc.

Ann. Giugno, Luglio.

Radicchiella selvatica it. - Cr. fétide fr.

(Barkhausia foetida DC.)

* Rostri tutti uguali.

C. setosa Haller Fil. (Crepis biennis Re-All.). Ovunque ai margini dei campi e dei prati.

Ann. Maggio, Giugno.

Radicchiella setolosa it. - Cr. hérissée fr.

Nel testo C. biennis Lin. dall' Allioni, e dal Murray. C. biennis Lin. è tutt'altra pianta; ha gli acheni senza rostro e nasce in Lombardia e nel Veneto. 
C. albida VilL. - Abbonda sopra Foresto : + comune anche nella Valle di Bardonêche.

Per. Giugno, Agosto.

Radicchiella biancastra it. - Cr. blanchâtre fr.

\section{GEN. 64. - Hiearmeinm LIN.}

1) Acheni brevissimi ( 2 millimetri circa) crenulati all'apice : pappo uguale a peli uniseriati : fori gialli.

* Rizoma serpeggiante stolonifero.

H. pilosella Lis. - Pilosella Off. - Ovunque nei siti aridi e nelle vie. Astringente.

Per. Maggio, Agosto. (Linguette rosseggianti disotto!.

Pelosella it. - Épervière piloselle fr.

Il decotto o estratto di H. Pilosella è stato vantato come un eccellente farmaco antimalarico nelle febbri intermittenti a tipo quartano, e nelle nevralgie ricorrenti. In Russia la pianta medesima è rimedio popolare contro la scrofola $A$ l'idrofobia (Cantani).

H. auricula Lri. (H. dubium RE dalla localiti). Nei pascoli erbosi.

Per. Giugno, Agosto.

Pelosella lattughina it. - Ep. auricule: Grande oreille de souris fr.

Nel testo H. dubium Lin. dal Willd. spec.

H. aurantiacum LiN. - Nel grande e nel piccolo Cenisio.

Per. Giugno, Luglio. (Fiori crocei).

Jeracio sussù it. - Ep. orangée fr. 
Rizoma discendente con o senza stoloni; oppure serpeggiante ma senza stoloni.

H. glaciale Lachen. (H. auricula Re dalla località) Sommità del Cenisio.

Per. Luglio, Agosto.

Pelosella orecchietta it. - Ep. des glaciers fr.

Cenisio a Ronche (Negri).

(H. angustifolium Hoppe).

H. sabinum Sebast. e Macri, Fl. Rom. Prodr. (H. praemorsum RE dalla località) - Cenisio, Musinè, ed anche presso Susa nei siti silvestri delle Blaccie.

Per. Giugno, Luglio.

Pelosella irsuta it. - Ep. en eyme fr.

Pascoli del Cenisio su le rive meridionali del lago (Bourier).

Nel testo: H. praemorsum Lin. dal Murray Syst. e dall'Allioni ch" è tutt'altra specie, nativa dei monti LombardoVeneti e Tirolesi, e sinonima di Crepis pracmorsa Tausch. (Bertol., Fl. it., Comp. della Fl. it. di Cesati, ecc.) E che trattisi qui del H. Sabinum fanno fede gli esemplari del Cenisio spediti dal Balbis al Bertoloni, e quelli da noi osservati nell' erbario dell'orto botanico di Torino.

H. florentinum Willdd. (H. cymosum Re-AlL.). -- COmune nei pascoli sabbiosi presso i torrenti.

Per. Maggio, Luglio.

Pelosella prataiuola it. - Ep. de Florence fr.

Alle falde della Sagra di San Michele, Re, append. IV, ad Fl. ped. inedita sotto H. praealtum (Colla Herb., vol. VII, p. 416).

Presso il torrente detto del Gravi a Condove (Re, app. II. ad Fl. ped. sotto Hier. piloselloides Vill.)

Nel testo H. cymosum Lin. dall'Allioni e dal Murr. Syst. specie diversa appartenente all'Ossola ed al Veneto. 
'2) Acheni muniti all'apice di un margine intero, lunghi 3-4 millimetri: peli del pappo ineguali biseriali : fiori gialli.

Rinnovamento dei cauli annuali su rosette autunnali persistenti svernate.

a) Squame dell'involucro regolarmente embriciate in piil serie: fori colorati in giallo più o meno carico.

H. amplexicaule Lin. - Nei siti sassosi del Cenisio, e presso Susa.

Per. Luglio, Agosto.

Geracio viscoso it. - Ep. embrassante fr.

Sagra di S. Michele (De Filippi).

H. saxatile ViLt. - Trovato dal chiar. Bellardi sul Cenisio.

Per. Giugno, Luglio.

H. saxatile fr. - Ep. des lieux pierreux fr.

Siti rupestri del Cenisio (Bell., App. ad. Fl. Ped., pag. 36).

H. villosum Lin. - Cenisio.

Per. Luglio, Agosto.

Geracio barbuto it. - Ep. velue fr.

Varia a foglie glabre nella pagina superiore H. flexuosum Walds et Kit. fra la Posta e la Ramasse verso il lago (Bonjean in Colla Herb.)

Altipiano del Cenisio (Negri).

Una forma di $H$. villosum sembra che sia $H$. cerinthoides che il Re riporta come pianta del Cenisio, e come specie Linneana dal sinonimo dell'Allioni.

H. cerinthoides Lin. è pianta di Corsica.

* H. glabratum Hoppe -- Altipiano del Cenisio (Negri).

Per. Giugno, Luglio.

Geracio a foglie di scorzonera it. - Ep. à feuilles de scorzonère $\mathrm{fr}$.

Avendo trovato nel Compendio della Fl. it. del Cesati ece. assegnata a questa specic (varietà del H. scorzoneraefolium 
Vill. per gl'illustri autori) tutt'altra località che il Cenisio, e le alpi piemontesi, interrogammo all'uopo per lettera il ch. Signor Negri, il quale gentilmente rispose:

"Il Hieracium glabratum Hoppe fu da me raccolto nelle vicinanze della Gran Croce, altipiano del Cenisio, e lo raccolse pure il Rosellini il 3 agosto 1863 nella stessa località.

"Sventuratamente tale specie sembra rara, giacchè, come vedo dall'erbario suo, il Rosellini ne raccolse come me un solo esemplare. »

H. alpinum Liv. - Sommità del Cenisio.

Per. Luglio, Agosto.

Radicchiella irsuta it. - Ep. des alpes fr.

y Halleri Косн $(\boldsymbol{H}$. hybridum Re-VILL.). - Occorse a Ignazio Molineri nei monti di Giaveno.

H. glaucum ALL. - Trovato da Ignazio Moliner nei siti sabbiosi lungo la Dora presso Cesana.

Per. Luglio, Agosto.

Geracio verdemare it.

\section{H. porrifolium Lin. - Nella Valle di Exilles.}

Per. Giugno, Agosto.

Geracio porrine it.

H. staticifolium VILL. - Comunissimo nei siti caldi e arenosi presso i torrenti e i fiumi.

Per. Giugno, Agosto.

Geracio livido it. - Ep. à feuilles de statice fr.

Altipiano del Cenisio (Negri). Sagra di San Michele.

* H. glanduliferum Hoppe. - Cenisio a l'Eau blanche (Negri) Cenisio a Ronche (Bouvier).

Per. Luglio, Agosto.

Geracio glanduloso it. - Ep. glanduleuse fr. 
b) Squame dell'involucro irregolarmente embriciate: le squame inferiori pii brevi rappresentanti in certa guisa un caliculo: fori 6 color giallo piu o meno carico.

H. tomentosum ALr. (Andryala lanata RE-LrN.). Siti alpestri sassosi del Cenisio e di Giaveno: dietro la Brunetta, e sopra il Borgo di Monpantero detto Monpantero Vecchio.

Per. Maggio, Giugno.

Geracio felpato it. - Ep. laineuse fr.

(H. lanatum Vill.).

H. murorum Lis. - Pulmonaria gallorum o Pulmonaria gallica Off. - Ovunque nei siti collinosi e montani. Rimedio disusato.

* H. vulgatum Fries - Musinè (Re, Fl. Tor. sotto H. Sylvaticum).

Per. Luglio, Agosto.

Geracio tardivo it. - Ep. des bois fr.

Rinnovamento dei cauli annuali su gemme primaverili, non già da rosette svernate, le quali mancano: fiori color giallo pith o meno carico.

H. prenanthoides Vill. ( H. spicatum RE-ALL.). Presso il torrente del Gravi a Condove, e nel Cenisio.

Per. Luglio, Agosto.

Geracio lattugaccio it. - Ep. à feuilles de Prenanthe fr. Malamente e forse per errore materiale nel testo $\mathrm{H}$. spicatum Lin.

H. sabaudum LiN. AlL. - Nei siti montani. Per. Agosto, Settembre.

Geracio autunnale it. - Ep. de Savoie fr. 
H. umbellatum Lin. - Siti sassosi a Condove ai margini delle selve presso il torrente del Gravi.

Per. Luglio, Settembre.

Geracio ombrellato it. - Ep. en ombelle fr.

Le specie di Hieracium sono in generale proteiformi, e soggette all'ibridismo. quindi talune di esse presentano alla determinazione difficolti grandissima. Le varie forme furono prese per tante distinte specie, e quindi la loro sinonimia divenne un vero laberinto.

Ordine LXVi. - Ambrosiacee (Ambrosiacene Link.).

\section{GEN. Manthium LIN.}

\section{X. strumarium LiN. - Lappa minor - Xanthium} Off. - Siti pingui intorno ai campi e le case. Subacre, amara: dà una tinta gialla.

Ann. Giugno, Luglio.

Bardana minore it. - Lampourde glouteron $\mathrm{fr}$.

Ordine LXVII. - Campanulacee

(Campanulaceae. Juss.)

A) Antere aderenti fra bro per la base.

\section{GEN. 1. - Jasione LIx.}

(Fiore ceruleo).

J. montana LiN. - Frequente nei siti secchi soleggiati cosi a Susa come nei pascoli aridi di Condove alla Roccea.

Ann. Maggio, Luglio.

Vedovella celeste it. - Jasione de montagne. Herbe z Midì $\mathrm{fr}$. 
B) Antere libere.

\section{Gen. 2. - Plnyternma Lin.}

1) Fiori disposti a spiga ovata prima della fioritura : cilindrica dopo.

* Fiori cerulei.

Ph. Michelii Willd. Bertol. Fl. it. (Phyteuma spicata Re-AlL.). Rapunculus spicatus Off. - Volgare nei prati alpini e subalpini, e ai margini delle selve. Rimedio disusato.

* c betonicifolium Косн. (Ph. betonicifolium VILL. Re, Fl. Tor.) Cenisio (Bouvier) - Musinè verso la Madonna delle Basse (De Filippi) Colle della Portia.

$y$ angustissimum Косн. (Ph. Michelii RE-ALL.). Cenisio.

Per. Luglio, Agosto.

Raponzolo di selva it.

Ph. Halleri Alu. - Frequente nei siti ombrosi del Cenisio, trovato da Felice Valle nei prati fertilissimi al luogo detto Echallier sopra il monte Fraisseu.

Per. Giugno, Luglio.

Raponzolo coda di volpe it. - Raiponce d'Haller fr. Musinè (Re, Fl. Tor. sotto $\mathrm{Ph}$, ovatem).

* Fiori bianco-giallicci.

* Ph. spicatum Lin. - Prati della Novalesa (Bonjean in Bertol.).

Per. Giugno, Luglio.

Raponzolo maggiore it. - Raip. en épi : Raip. sauvage fr. 
2) Fiori disposti in capitolo globoso o quasi prima e dopo la fioritura.

* Brattee larghe numerose uniformi.

Ph. pauciflorum Lin. - siu le vette più alte del Cenisio.

Per. Luglio, Agosto.

Raponzolo spatulino it. - Raip. i petite tête fr.

Cima d'Eau blanche (Negri).

* $\beta$ Bertol. (Ph. globulariaefolium Hoppe e Sterub.) Ccnisio (Bouvier).

* Ph. hemisphaericum Liv. - Cenisio (Balbis, Molineri in Bertol.).

Per. Luglio, Agosto.

Raponzolo gramignolo it. -- Raip. hémisphaerique $i$.

$\mathrm{Ph}$. orbicolare LIx. - Frequente nei prati alpini e montani.

Per. Giugno, Agosto.

Raponzolo montanino fr. - Raip. orbiculaire fir. Altipiano del Cenisio (Negri).

" Brattee anguste scarse disuguali.

Ph. Scheuchzeri Alt. - Rupi ombrose presso Giaveno.

Per. Luglio, Agosto.

Raponzolo cornuto it.

\section{Gen. 3. - Campamulla LiN.}

(Fiori a tinta azzurra piu o meno carica, o rar. cerulea bianchiceia o gialla)

1) Seni del calice muniti di un'appendice riflessa.

\section{C. alpestris Alt. - Cenisio a Ronche.}

Per. Luglio, Agosto.

Campanella d'alpe it. - Campanule d'Allioni ir. 
Cenisio (Molineri, Balbis, Bonjean. Bertol.) Cima d`Eau blanche (Negri). Assietta.

(Campanula Allionii Vill.).

C. barbata LiN. - Nei prati e nei pascoli montani e alpestri.

Per. Giugnio, Agosto.

Campanella barbuta it. - C. barbue fr.

Sopra la cittadeila salendo al torrente del Vallon tra Villarfocchiardo e il piano delle Cavalle (Bruno: Sagra di S. Michele (De Filippi). Assietta.

C. sibirica LiN. - Orunque nelle rupi calde solegrgiate da Susa ad Alpignano: specialmente a Monpantero.

Bienn. Maggio, Giugno.

Campanella orecchiuta it.

2) Seni del calice sprovvisti di appendici.

Fiori peduncolati disposti a pannocchia a racemo, o solitari.

C. cenisia LiN. - In copia sul Cenisio a Ronche.

Per. Luglio, Agosto.

Campanella sdraiata it. - C. du mont Cenis fr. Località medesima (Negri).

${ }^{*}$ C. persicifolia Lin. - Dintorni di Susa. (Arogadro in Colla Herb., vol. VII, pag. 417).

Per. Giugno, Luglio.

Campanella: Giglio di San Luigi it. - C. il feuilles de pêcher fr. 


\section{Rapunculus Lix. - Rapunoulus arvensis o Escu-} lentus Off. - Rampon vern. - Qua e là nei siti incolti e presso le vie. La radice è commestibile in insalata.

Ann. Giugno, Agosto.

Raponzolo it. - C. raiponce fr.

C. elatines Lin. - Frequente nelle rupi alpestri sli Mocchie nella contrada detta di Barmoscel, e sopiosissima anche nei rnonti di Giaveno.

Per. Luglio, Agosto.

Campanella con foglia di ortica it. - Giolat vern.

Cenisio (Re in Bertol.)

Nei monti di Givoletto prossimi al Musinè vegeta una fpiccata varieti di questa specio, C. elatines $\beta$ glaberrim:? DC. fil, ivi trovata la prima volta da Chiuso e De Filippi riatrdinieri del R. Orto Botanico di Torino. Essa fu ennsiderata cone specie dal Del Ponte e dal Gras, e chiamata Campranula subalpina (Vedi l'ine salive alla torre d' Onarde. Fratelli Bocca, Torino, 1873, pag. 40 a 42).

a. pusilla Haexk. (C. coespilosa Re-Vict.). - Siti sassosi delle alpi.

Per. Luglio, Agosto.

Campanella soldanella it. - Erba strucaira vern. C. fluette fr.

Blaccie e Gravere (Negri). Assietta.

C. roundifolia Lra. - Siti montani ed alpestri.

Per. Giugno, Agrosto.

Campanella soldanella it. -- Violetta piranidal rern. C. $\grave{a}$ feuilles rondes $\mathrm{fr}$.

Musine (Re, Fl. Tor.). Blaccie e Gravere (Negri). 
* C. Inifolia Haenk. - Cenisio (Bonjean in Bertol.). Sagra di S. Michele.

Galdensis (C. valdensis Alz.). - Cenisio (Bonjean in Bertol. e Colla).

Per. Lugiio, Agosto.

Campanella ginestrina it. C. it fouilles de lin fr.

C. rhomboidalis Lix. - Ovunque nei prati alpini. Per. Luglio.

Raponzolo romboidale it. - C. rhomboïdale fr.

Conisio (Re, Bonjean in Bertol.) Cenisio, ricinanze del lago (Negri).

* E. Erinus Lin. - Alle Blaccie Cesati, Tegrri. Ann. Maggio, Giugno.

Campanella dei muri it. - C. érine fr:

C. rapunculoides LIN - Uvularia minor Off. Campi montani. Farmaco disusato.

Per. Luglio, Agosto.

Campanellir sorpeggiante it. - C. fullse raponee it.

C. bononiensis Irn. - Abbouda a Condove nel sito detto Grangiettil e nella ralle di Exilles.

Per. Luglio, Agosto.

Raponzolo di Bologna it. - C. à petites fleurs fr.

C. trachelium Lx. - Campanula, Tracheliun o Uvularia Dff. - Orunque nei pascoli. Rimedio disusato.

Per. Luglio, Agosto.

Imbutini it. -- Spinas servaj reru. - C. gantelée, Gant de Notre Dame fr.

3 dasycarpa Gren. e Godr. (C. dasycarpa DC. fil.) con lia specie. 
C. Iatifolia Lin. - Trovata da Ignazio Molineri presso la fabbrica del marmo: da Felice Valle presso il forte diroccato di Exilles.

Per. Luglio, Agosto.

Campanella campanone it. - C. it larges feuilles fr.

* Fiori sessili o quasi sessili in spiche, o capitoli.

C. spicata Lin. Mant. - Cenisio alla posta: a Susa nelle rupi di Monpantero e della Coà sopra Foresto, e copiosissima nei prati del Collet verso Fenestrelle.

Bienn. Giugno, Luglio. (Fiori azzurri).

Raponzolo spigato it. - C. en epi ir.

C. thyrsoides Lin. - Nel Cenisio presso la Ramasse.

Bienn. Luglio, Agosto. (Fiori gialli).

Campanelle gialle it. - C. on thyrse fr.

C. glomeraia Lis. - Comunissima nei prati montani ed alpestri.

Per. Maggio, Giugno. (Fiori azzurii).

Campanelle a capolino it. - C. agglomerée fr.

3 aggregata (C. aggregata Willd.) Cenisio (Cesati).

Benchè non faccia propriamente parte della nostra Flora, tuttavia notiamo una Campanulacea che vegeta ai suoi confini, Adenophora lilifolia Ledeb. (Campanula alpini Lin.), tenuta sino a pochi anni sono come propria delle alpi Venete.

Questa specie vegeta nei monti di Giroletto, prossimi al Musinè e chi vuol rintracciarla prenda a Givoletto la via del Pilone, e percorra la cresta dei monti verso Varisella. (De Filippi). 
GEN. 4. - Speculapia HEISTER.

S. speculum DC. PRod. (Campanula speculum Re-LiN.). - Speculum veneris o Avicularia Off. - Ovunque nei campi. Rimedio disusato.

Ann. Maggio, Giugno. (Fiori purpureo-violacei).

Spechio di Venere it. - Pense servaj vern. - Speculaire miroir de Venus fr.

S. falcata DC. Prod. (Campanula hybrida Re-A.tc.). Trovata nelle messi presso Susa dal chiaro Allioni.

Ann. Maggio. (Fiori purpurei).

Specchietto falcato it. - S. hybride fr.

Nel testo C. hylrida Lin. dal sinonimo di Allioni, specie diversa e non appartenente alla nostra Flora.

ORune I.TII. - TACCinde (Vaccinicae DC.).

\section{GEN. acecำำำ LIN.}

Foglie caduche: corolla ovata o globosa.

V. myrtilus Lis. - Ambrune vern. - Abbonrla nei siti montani ed alpini.

Sufirut. Maggio, Giugno. (Foglie minutamente seghettate). Mirtillo it. - Airelle myrtille fr.

Musinè (Re, Fl. T'or.).

Y. uliginosum Itr. - Cenisio e altri pascoli alpini.

Suffrut. Giugno, Luglio. (Foglie intere).

Baggiolo bianco it. - Air. fangeuse fr.

Colle della Portia a Condove. Colle della Russa a Giaveno. 
* Foglie persistenti : corolla eampanalata.

V. vitis idaea Lrs. - Qua e là intorno alle selve e nei pascoli montani ed alpestri. Copiosa nelle alpi di Mocchie nel sito detto Pianfei.

Suffrut. Maggio, Luglio.

Vite di monte it. - Air. ponctuée: Herbe rouge fr.

Sagra di S. Michele (Gras).

\section{Ordine LIX. - Frictane (Ericineale Dest.).}

Gen. 1. - Aretogtaplaylos Anan..

Arc. alpina Sprengel. (Arbulus alpima Re-LiN.) -Siti freddi posti a nord sul Cenisio.

Suffrut. Giugno, Luglio. (Foglie inegualmente e minut:mente seghettate : fiori bianchi).

Rossello alpino it. - Arbousier des alpes fr.

Arc. officinalis Wmin. e Grab. (Arbutus wva Ursi ReLin. - Uva ursi Off. - Copioso nel Cenisio, nella valle di Exilles, e nelle alpi di Giaveno. Foglie amaro-stittiche, astringenti, diuretiche.

Suffrut. Maggio, Giugno. (Foglie interissime: fiori carnicini).

Uva orsina it. - Arb. busserole, Raisin d'ours fr.

Musinè (Gras).

Le foglie di uva ursina contengono molto acido tannico, e riescono senza dubbio astringenti. In decozioni sono adoperate con successo nei catarri vescicali. 


\section{Gex. 2. - Calluma SALisb.}

G. vulgaris SAlisb. (Erica vulgaris Re-LiN.). - Erica Off. - Bru vern. - Comunissima nei siti montani e subalpini. Rimedio disusato.

Suffrut. Luglio, Agosto.

Erica it. - Calluna bruyère fr.

GEN. 3. - zalea LIN.

A. procumbens Lin. - Copiosa nei siti freddi del Cenisio, e nei monti di Giaveno.

Suffrut. Luglio, Agosto. (Fiori rosei).

Azalea bosso alpino it. - Azalée couchée fr.

\section{GEN. Hhododemdrom LiN.}

R. ferrugineum I.IN. - Chamoerodendron o Nerium alpinum 0ff. - Brussei vern. - Volgare in tutte le alpi. Narcotico, sospetto.

Frutice. Giugno, Agosto.

Rododendro alpino it. - Rosage herissé fr.

Le foglie del Rododendro alpino infuse sono diuretiche: c purgative, e prese ad alte dosi riescono narcotiche. Musinè (Re, Fl. Tor.).

Ordine LX. - Prror.Acel (Pyrolacele Lindl.) Gen. Pyrola LiN.

*Fiore grande, bianco, solitario.

P. uniflora Lrs. - Cenisio (Bonjean in Bertol.). Per. Giugno, Luglio.

Piroletta soldanina it. - Pyrole uniflore fr. 
* * Fiori in racemi.

P. rotundifolia Lrs. - Pyrola Off. - Nel Cenisio. Astringente.

Per. Giugno, Luglio. (Fiori bianchi).

Limonio montano it. - - P. à feuilles rondes fr.

Farmaco disusato.

P. secunda LIN. - Cenisio.

Per. Giugno, Luglio. (Fiori verde-bianchi piccoli).

Ambrosia montana it. - P. unilaterale fr.

* P. minor Lin. - Cenisio (Bonjean in Bertol.)

Per. Giugno, Luglio. (Fiori bianco-rosei).

Limonio montano minore it. - P. à style court fr.

Nota: - Nel foglio antecedente alla pagina 221 invece di Drdine LITI e LXVII - leggi: Ordine LVI e LVII. 


\section{DIVISIONE TERZA.}

\section{Corolliflore - (Corolliflores)}

Ordine LXI. - Agrilogliace (Aguifoliaceae .DC:

\section{Gen. Hex Lin.}

1. Aquifolium Lrx. - Agrifolium o Aquifolium Off. - Abbonda nelle selve montane di Giaglione, presso Le Ferriere, e sopra Foresto. Foglie amare ed astringenti.

Alberetto. Maggio, Giugno. (Fiori bianchi). Agrifoglio it. - Houx commun, Agrifon Ir: Farmaco disusato.

Oruine Lxir. - Oleacli: (Oleaceae Lindl.). GEN. $1^{v}$ - Ligustrum LIN.

L. vulgare Lis. - Ligustrum Off. - Ovunque nelle siepi e nelle selve. Foglie astringrenti. Le bacche danno un color nero.

Frutice. Maggio, Giugno. (Fiori bianchi).

Ligustro it. - Troëne commun fr.

Come farmaco è disusato: le bacche sono adoperate dis taluni ad imposturare il colore del vino. 


\section{GEN. 2. - Fraximats LIN.}

F. excelsior Liv. - Fraxinus Off. - Frasso vern. - Ovunque nei siti montani. Corteccia amaricante: antisettico. Semi diuretici.

Arbusto. Maggio, Giugno.

Frassino it. - Frêne elevé fr.

Farmaco disusato.

Ordine LXiII. - AsclepradeE (Asclepiacleae R. Bromn.)

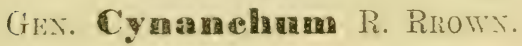

Cy. Vincetoxicum R. Browi. Asclepias Vincetoxicum Re-Lin.) - Asclepias, Vinceloxicum, Hyrundinaria $0 \mathrm{ff}$. - Frequente nei pascoli montani ed alpestri, ed ai margini delle selve. La rarlice is sub-relenosa, dinretica.

Per. Giugno, Agrosto.

Asclepiade vincetussico ir. - Dompte-renin officinale ti.

Dí un odore poeo grato: is irritante; non i pir adoprerato in farmacia.

\section{ORdine LXIV. - APOCINEE \\ (Apocyneae R. Bronon.) \\ GEN. Viner Lin.}

\section{V. minor Lrs. - Vincae pervincae herba Off.} Volgare nelle selre. Si prescrive nei mali di grola sotto forma di gargarismo. el anche come gengivario.

Per. Maggio.

Pervinca, Fior da morto it. - Pervenche couchée fr.

Pressochè abbandonata dalla farmacia di oggi. 
Ordine LXV. - Genzianacee.

(Gentianecue Juss.)

A) Foglie alterne ternate.

GEN. 1. - Premy mothes.

\section{M. trifoliata LIN. - Trifolii fibrini o Trifolii pa-} lustris herba 0ff. - Presso i laghi di Avigliana,

e nei prati irrigui di Almese. Amara, astringente, e corroborante.

Per. Maggio, Giugno. (Fiori rosei).

Trifoglio fibrino it. - Menyanthe trefle d'eau fr.

Il trifoglio fibrino è un ottimo amaro meno nauscoso, e dispendioso della Quassia, ed è desiderabile che la farmacia lo tenga in maggior conto, almeno nelle cuntrade ove esso vegeta spontaneamente.

B) Foglie opposte.

Gen. 2. - Chlord LiN.

(Fiori gialli : calice 8-partito)

Ch. perfoliala LrN. Syst. nat. - Siti umidi di Fossimagna, di Prato Molle, di Monpantero, e presso la Dora.

Ann. Luglio, Agosto.

Centaurea gialla it. - Chlorette perfoliée fi.

\section{GEN. 3. - Swertia Lin.}

(Fiori bruno-violacei : calice 4-5 partito)

3. perennis Lin. - Prâti fređdi del Cenisio.

Per. Luglio, Agosto.

Genziana stellata it. - Swertie vivace fr.

Prati presso il Lagao del Cenisio, e alla Ramasse. Boujean in Bertol.). 


\section{GEN. 4. - Gemtiama LIN.}

3) Fauce della corolla non barbata

" Corolle gialle e gialliccie punteggiate di scuro in G. punctata.

G. lutea LiN. - Gentianae majoris radix Off. Gensana vern. - Frequente nei prati alpini. Amarissima, stomatica, antielmintica.

Per. Luglio, Agosto.

Genziana it. - Gentiane jaune, Grande gentiane fr.

La radice di yuesta specie è un ottimo amaro tonico. Non ¿ anticlmintica, nè guarisce le febbri malariche come una volta si credeva.

G. punctata Liv. - Abbonda a mezzogiorno del Iago del Cenisio e sull'Assietta. Radice amara, stomatica.

Per. Luglio, Settembre.

Genziana rossigna it. - G. ponctuée fr.

Monti di Bussoleno, alla Balmetta.

- Corolle azzurre alneno internamente: rar. bianche.

G. cruciata Lis. - Raccolta da me in copia nei prati sopra La Losa.

Per. Giugno, Luglio. (Corolla spessissimo 4-fida!.

Genziana minore it. - G. croisette fr

(Alla Novalesa Bonjean, Balbis in Bertol.)

G. asclepiadea LrN. - Siti freddi ed umidi delle alpi : copiosa intorno al lago del Cenisio.

Per. Luglio, Settembre.

Asclepiade it. - G. asclépiade fr.

Alpi della Novalesa regione Tourdupi (Signora ChiapussoVoli). 
G. pneumonanthe LIN. - Io la raccolsi nei siti umidi di Prato Molle, ed Ignazio Molineri a Caselette.

Per. Luglio, Settembre.

Genziana minima it. - G. pneumonanthe, pulmonaire des marais $\mathrm{fr}$.

G. ciliata Lin. - Cenisio, e sopra Condove andando verso Mocchie.

Per. Luglio, Settembre.

Genzianella sfrangiata it. - G. ciliée fr.

(Corolla 4-fida a lobi cigliato-frangiati).

Alpi della Novalesa a Tourdupi (Signora Chiapusso Voli).

G. acaulis Lix. - Frequente nel Cenisio e in tutti i pascoli montani ed alpini.

y) parvifolia (irex. e Govr. ( G. alpinn Re-Vilt.). Trovata dal chiaro Bellardi nei prati secehi del Cenisio.

Per. Giugno, Luglio. (Fiori grandi splendidi).

Genziana maggiore it. - G. à tige courte fr.

G. bavarica Liv. - Siti nmidi e freddi del Ce. nisio sopra la Ramasse e nelle alpi di Giaveno.

Per. Luglio, Agosto.

Genzianella azzurra it. - $G$. de Bavière fr.

G. verna Lix. - Volgare in tutte le alpi.

Per. Maggio, Agosto.

Genzianella celeste it. - G. printaniere fir.

G. utriculosa LiN. - Cenisio : in Valle di Exilles nella sommita del monte detto Les Crevasses.

Ann. Giugno, Agosto. (Calice gonfio carenato-alato). Pettimborsa alata it. - G. à calice renflè fr. 
G. nivalis IIN. - Cenisio ed altri pascoli alpini. Ann. Luglio, Agosto. Genzianella fugace it. - G. des neiges fr.

2) Fauce della corolla barbata.

G. glacialis Vit.. (Gentiana nana Re-ALt.). - Trovata da Ignazio Molineri nei paseoli freddi del Cenisio, ma è rara e fugace.

Ann. Luglio, Agosto (Corolla spesso 4-fida). Genzianella gåmbata it. -- G. délicate fr.

Cenisio a Ronche (Negri).

Nel testo G. nana Lin. probabilnente per erore materials.

G. amarella Lr:. Gentiana campestris Re soc. Bertol. - Pascoli montani ed alpestri. Copiosa nelle alpi di Mocchie al sito detto La Frère sopra Pianfei. Ann. Giugno, Agosto. (Corolla 5-fida). Amarella it. - G. amarelle fr. (Vedi Bertol., Fl. it., vol. 3, pag. 97 c 99).

* G. campestris Livi. - Sionti prossimi al Musinè (Re, Fl. Tor.). Cenisio (Boujean in Bertol.. Assietta.

Ann. Luglio, Agosto. (Corolla quadrifida). Amarella prataiola it. - G. des champs. $\mathrm{fr}^{2}$.

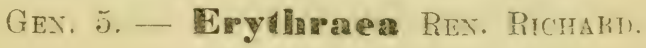

(Fiori carnicini, talvolía bianchi).

E. centaurium Pers. (Gentiana cenlauriem Re-Lix.) - Centaurii minoris horba off. - Centaura minur vern. - Ovunque nei pascoli lungo la Dora: amara e corroborante.

Bienn. Luglio, Agosto.

Centaurea minore it. - Erythrée centaurée fr. 


\section{8}

La Centaurea minore $i$ anara tonica come il trifoglio fibrino ed ha inoltre, secondo taluni scrittori di materia modica, proprietà di blando purgativo.

\section{Ordine LXVi. - Convolvulacee}

(Convolvulaceae Juss.)

\section{Gen 1. - Comedrubes Juss.}

C. sepium Liv. - C. major albus Off. - Nelle siepí Il succo delle foglie, spremuto, ì acre e amaricante. Per. Giugno, Agosto.

Vilucchio maggiore it. - Liseron des haies fr. Farmaco disusato.

C. arvensis Lrx. - Convolvulus minol o Smilaz larvis minor Off. - Nei campi: infestissimo. Farmaco disusato.

Per. Giugno, Luglio.

Vilucchio minore it. - L. des champs fr.

\section{Gen. 2. - Cassenta Lin.}

C. curopaea Lin. - Cuscuta cassuta o Epythymum Off. - Frequente nei trifogli, e presso Susa ab-bonda alle Blaccie. Amaricante.

Ann. Luglio, Agosto.

Cuscuta it. - Cuscute i grandes fleurs fr.

Farmaco disusato. 
Ordine LXVII. - Borraginee. (Boragineae Desv.)

Sezione 1 $^{2}$ - Fatee della corolla provista di sęuame. Ie quali soverate me chiudono il tabo.

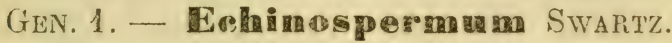

E. lappula Lens. (Myosotis Iappula RE-LiN.). Volgare nelle vigne, e nei campi sterili.

Bienn. Laglio, Agosto. (Fiori cerulei)

Lappolini it. - Bardanette faux myosotis fr.

Gen. 2. - Cymogilossurar Lix.

C. officinale Lriv. - Synoglossae radix ef herba Off. - Non rinvenni mai nei dintorni di Susa questa pianta ch'è frequentissima ovunque in Picmonte. Trovasi però ai margini dei campi tra S. Ambrogio e Avigliana, presso S. Antonino e lungo la via andando al Monte S. Benedetto di Villarfocchiardo. Subnarcotica.

Bienn. Maggio, Giugno. (Fiori rossi-violacei).

Cinoglosso it. - Cynoglosse officinale fr.

Il Cinoglosso è tenuto da terapisti moderni come astringente e come tale è adoperato. F insussistente la proprietio narcotica attribuitagli dal Re.

\section{GEN. 3. - Myosdis LIN.}

M. intermedia LiNk. (M. scoppioides Re a LiN., e Myosotis arvensis Re-ALL.). - Frequente nei campi e nei pascoli. Abbonda presso la Brunetta, e nei pascoli alpini di Mocchie.

Per. Giugno, Agosto. (Fiori cerulei).

Centonehio selvatico it. - Boca d'fea rern. - Myosotis intermediaire $\mathrm{fr}$. 
M. palustris Wrutr. - Abbonda presso le fonti. I'er. Giugno, Agosto. (Fiori di un ceruleo splendido). Taleo celeste it. - My. des marais fi. (M. scorpioides 3 palustris Lin.) Nel testo M. palustris Lin.

\section{* M. aipesiris Schant. - Cenisio (Bourier).}

Per. Luglio, Agosto. (Fiori azzurri odorosi).

Orecchia di topo alpina it. - My. des alpes fr. (Myosotis pyrenaica Pourr.).

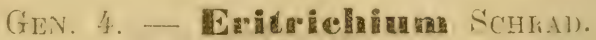

E. nanum Schrat. (Myosotis nana Rr-ALL.-Til.. ). - Abbonda su le alte ciuce diel Cenisio percosse dal vento.

Per. Luglio, Agosto. (Fiori di un ceruleo splendidissimo). Orecchia di topo camnt:1. - Eritrichio nano it. - Eritriche naine fre.

Pianta nana cespitosa beliissina, che il (iras con lelier epiteto chiamó Zaffiro dei ghiacciai.

Cima d'Eau blanche (Negri).

\section{GEN. 5. - Sperago LiN:}

A. procumbens Lrx. - Intormo alle case e nelle siepi; presso Susa alle Blaccie.

Amn. Maggio, Giugno. (Fiori purpureo-cerulescenti .

Lingua di bue it. - Rapêtte couchée fr.

\section{GEN. 6. - Lyeapis Lix.}

L. arvensis Lrx. - Nelle rigne presso Susa e nei campi a Condove.

Ann. Giugno, Agosto. (Fiori cerulei).

Lingua di bue it. - Lycopside des champs fr. 


\section{GEN. 7. - Symphitum LiN.}

\section{S. officinale LIN. - Symphitum o Consolida major} Dif. - Frequente nei prati umidi e pingui. Presso Susa nella Contrada San Saturnino. Radice emolliente.

Per. Maggio, Giugno. (Fiori bianchi, rosei o violacei).

Consolida maggiore it. - Consoude officinale fr.

Ebbe un giorno gran rinomanza come rimedio nelle malattie di petto: oggi è poco usato nella farmacia razionale, ma è rimasto un rimedio popolare.

S. \{uberosum LiN. - Nei siti montani ombrosi pingui.

Per. Aprile, Maggio. (Fiori gialli).

Consolida tuberosa it. - C. tubéreuse fr.

* Gen. 8. - BBonrago Lin.

* B. officinalis Lin. - Coltivata.

Ann. Giugno, Luglio.

Borraggine it. - Bouras vern. - Bourrache officinale fr.

Sez. $\mathfrak{z}^{2}$ - Fauce della corolla glabra o pubescente ma senza squame.

GeN. 9. - Cithospermum LiN.

L. officinale Lrs. - Lithospermum o Milium solis Dff. - Ovunque nei pascoli. Farmaco disusato.

Per. Maggio, Luglio. (Fiori verde-bianchi).

Litospermo it. - Gremil officinal fi.

L. arvense Lin. - Volgare nei campi.

Ann. Aprile, Giugno. (Fiori bianchi, rar. azzurri).

Strigolo selvatico it. - Gr. des champs fr. 


\section{L. purpureo-coeruleum LiN. - Lithospermum repens Off. - Nelle selve. Farmaco disusato.}

Per. Maggio, Giugno. (Fiori prima rossi poscia azzurri). Miglialsole maggiore it. - Gr. violet fr.

\section{GeN. 10. - Eelloingm LiN.}

Ech. vulgare e off. - Frequente nei siti aridi e sassosi.

Bienn. Giugno, Agosto. (Fiori cerulei rar. rosei o bianchi). Buglossa selvatica it. - Vipérine commune fr.

Ech. italicum Lin. - Trovato da Valle lungo la via presso Chiomonte. (All., Fl. Ped., I, pag. 52).

Bienn. Giugno, Luglio. (Fiori bianco-rosei o azzurrognoli). Echio it. - V. d'Italie fr.

\section{GEN. 11. - Pulmomatra LIN.}

P. angustifolia LiN. e Off. - Nelle selve montane. Amaricante.

Per. Aprile, Maggio. (Fiori prima rossi poi violacei).

Borrana celestina it. - Pulmonaire à feuilles éroites fr.

P. officinalis Lin. - Pulmonariae herba 0 off. Niente di più volgare nelle selve. Virtù della pre. cedente.

Polmonaria it. - P. officinale fr.

Tenute una volta per amare, astringenti: oggi pressochè dimenticate. 


\section{Gen. 12. - Onosma Lin.}

0. stellulatum WALDS et Krt. (0. echioides RE $\approx$ LIN.). - Anchusa lutea Off. - Volgarissima nelle rupi soleg'giate di Monpantero, Foresto, Bussolino, Chianoc e Borgone. Rimedio disusato.

Bienn. Giugno, Luglio. (Fiori gialli).

Viperina stellata it. - Orcanette fausse vipérine fr.

\section{GEN. 13. - Mellotropian.}

H. europoeum Lin. - Frequente nei campi. Ann. Luglio, Agosto. (Fiori bianchi o debolmente violacei). Eliotropio it. - Héliotrope d'Europe fr.

\section{Gen. 14. - Cerinthe LIN.}

C. minor Lrs. - Ovunque nel Cenisio.

Ann. Giugno, Agosto. (Fiori gialli).

Erba tortora dei grani it. - Melinet des alpes fr.

Ordine LXVIII - Solanacee (Solaneae Juss.)

Gen. 1. - Solamum Lin.

S. nigrum Lis. - Solani hortensis herba Off. Erba morella vern. - Frequente ai margini dei campi, e nei ruderi pingui. Velenosa.

Ann. Maggio, Settembre.

Morella it. - Morelle noire fr.

Disusata in medicina, la morella serve oggi alla preparazione della Solanina, principio attivo narcotico di alcune Solanacee, scoperta dal Desfosses nel 1821 appunto nelle bacche di questa specie. (Cantani). 


\section{S. dulcamara Lin. - Dulcamara o Solanum scan.} dens Off. - Ovunque nei siti umidi presso le acque stagnanti. Diuretica.

Frutice rampicante. Giugno, Luglio.

Dulcamara it. - M. douce-amère fr.

Ebbe un giorno gran fama medicinale come diuretica, oggi questa è di molto scemata. Il suo principio attivo è la Solanina di cui abbiamo fatto cenno poco innanzi. L'abbandono assoluto di questo celebre farmaco non è del tutto giustificabile (Cantani).

* S. tuberosum Lin. - Patata, Porn d'tera vern. Originaria d'America. Coltivata. Notissimo alimento in ispecie delle classi popolari. Qui cade in acconcio di far menzione di altre Solanacee esotiche naturalizzate tra noi e coltivate negli orti.

Lycopersicum esculentum Mill. - Pomidoro it. - Tomatica vern. : originaria d'America.

Capsicum annuum Lin. - Peperone it. - Pevron vern. : originario d'America.

Melangena teres MiLı. - Petronciana, Melenzana it. - Melensana vern.: originaria dell'India.

\section{Gen. 2. - Physallis LIN.}

Ph. alkekengi Lrn. - Alkekengi, Solanum vescicarium o Solanum halicacubum Off. - Frequente negli spineti e nelle siepi. Bacche diuretiche.

Per. Giugno, Luglio. (Bacche rosse chiuse nel calice gonfio).

Palloncini it. - Coqueret alkekenge fr.

Rimedio poco o niente usato dalla farmacia recente. 
GeN. 3. - Hyoseyamus Lin.

\section{Hy. niger Lis. - Hyoscyamus o Jusquiamus niger} Off. - Abbonda nei ruderati pingui, e nei siti suburbani. Velenoso.

Bienn. e Ann. Giugno, Luglio.

Giusquiamo nero it. - Jusquiame noire: Potelée fr.

Il Giusquiamo ha conservato il posto nella farmacia moderna e si amministra come calmante: il suo principio at. tivo è la josciaminina.

\section{Gen. 4. - Batura Lin.}

D. stramonium Lin. - Stramonium Off. - Nelle vigne di Susa esposte a mezzogiorno presso il forte diruto di Santa Maria nel sito detto la Roccetta. Velenoso, narcotico.

\section{Ann. Luglio, Agosto.}

Stramonio it. - Datura stramoine, Endormie, Herbe du diable, Pomme epineuse fr.

Lo Stramonio agisce come il Giusquiamo; le foglie secche fumate in modiche ed ordinate dosi, calmann l'asma sia ch'essa derivi da disturbi puramente nervosi, sia che nasca da catarri bronchiali.

\section{Ordine LXIX - Verbascee (Verbasceae Bartl.) \\ Gen. 1. - Verloaseram Lin. \\ - Fiori gialli, rar. bianchi. \\ a) Filamenti bianco-barbati.}

\section{V. thapsus Lix. - Thapsi barbati o Verbasci} flores 0ff. - Fior d' Luvion vern. - Fiori emollienti. - Qua e là nei pascoli e nei siti incolti.

Bienn. Luglio, Settembre.

Verbasco it. - Molêne bouillon blanc fr. 
Sta tra i rimedi mucilaginosi popolari, come talune delle seguenti specie.

V. phlomcides Lin. (V. thapsoides Re-All.). - Ai margini delle selve.

Bienn. Giugno, Settembre.

Barbarastio it. - M. phlomida fr.

Vie del Cenisio (Compendio della Fl. it. del Cesati, ecc.).

Nel testo V. thapsoides Lin. dall'Allioni (Vedi Bertol., Fl. it., vol. II, pag. 576 e Gren e Godr., Fl. Fr., vol. II, pag. 549).

y. Iychnitis Lin. - Ovunque nei pascoli. Emolliente.

Bienn. Giugno, Agosto. (Fiori gialli, oppure bianchi V. lychnitis $\beta$ album Koch).

Verbasco lichnite it. - M. lychnite fr.

* V. montanum SCHRAD. - Cenisio, nella valle di San Nicola (Bonjean in Bertol.).

Bienn. Giugno, Settembre.

Barabasco montanino it. - M. de montagne fr.

b) Filamenti purpureo o violaceo-barbati.

V. nigrum LIN. -- Ai margini delle selve. Radice emolliente.

Bienn. Giugno, Agrosto.

Verbasco nero it. - M. noire fr.

V. blattaria Lrv. - Blattaria lutea Off. - Qua e là nei pascoli umidetti e presso $i$ fossi. Farmaco disusato.

Bienn. Giugno, Agosto. (Corolla gialla col fondo purpureo; talvolta bianca).

Polline it. - M. blattaire fr. 
* Fiori violacei o rosso-scuri.

V. phoeniceum Lin. - Abbonda nei pascoli aridi ( declivi di Borgone al sito detto Pietra Cullera. Bienn. Giugno, Luglio.

Polline porporino it. - M. pourpre fr.

\section{A Caselette.}

Il genere Verbascum va soggetto in sommo grado all'ibridismo, e le forme ibride sono moltissime, e di non facile determinazione.

\section{GEN. 2. - \$erophularia Lin.}

*Foglie semplici seghettate o crenate.

S. nodosa Lin. - Qua e là nei siti ombrosi e pingui incolti. Carminativa, antielmintica.

Per. Giugno, Agosto.

Scrofolaria maggiore it. - Scrofulaire noueuse fr.

S. aquatica Lis. - Ovunque lungo i fossi, e presso le acque a lento corso.

Per. Maggio, Gingno.

Scrofularia it. - S. aquatique fr.

* Foglie pinnate e bipinnate.

S. canina LIN. - Comunissima nei pascoli sassosi ed arenosi.

Per. Maggio, Giugno. (Foglie pinnate).

Ruta canina it. - S. canine fr.

* S. Hoppii Koch. - Cenisio (Bonjean in Bertol.).

Per. Luglio, Agosto. (Foglie pinnate e bipinnate).

Scrofularia coriandolina it. - S. d'Hoppii fr.

Le specie di Scrofularia notate dal Re come medicinali sono oggi fuori uso. 
Ordine LXX - Antirrinee (Anthirrineae Juss.)

\section{Sez. ${ }^{2}$ - Corolla bilabiata a fauce chiusa e gronfia alla base.}

\section{GEN. 1. - Antipolnimum LIN.}

\section{A. maius Lin. - Antirrhinum o Caput vituli Off.}

- Nelle rupi presso la Brunetta e nelle mura vecchie di Avigliana.

Per. Maggio, Giugno. (Sepali ovali più corti della corolla). Antirrino: Bocca di leone it. - Muflier à grandes fleurs: Mufle de-veau fr.

A. orontium Lin. - Nei vigneti e nei campi ma. cilenti. Abbonda nei campi a Condove nel sito detto la Grangietta presso la Roccea.

Ann. Maggio, Agosto. (Sepali angusti lanceolati, uguali alla corolla o più lunghi).

Gallinella it. - M. rubicond: Tête de Mort fí.

Sez. $:^{n}$ - Corolla bilabiata a fauce piu o meno chiusa speronata alla brse.

\section{GEN. 2. Linaria JUSS.}

- Fauce della corolla completamente chiusa.

L. alpina Mirt. (Antirrhinum alpinum Re-LiN.). Frequente all'Assietta, e nel Cenisio: discende in pianura e trovasi nei siti sassosi lungo la Dora. Ann. Giugno, Agosto. (Corolla cerulea; gobba del palato crocea).

Linaiuola d'alpe it. - Linaire des alpes fr. 
L. genistaefolium MuL. (Antirrhinum genistifolium Re-Lin.). - Abbonda a Susa nei siti sassosi presso la Dora, e nei siti medesimi a San Lazzaro.

$\beta$ arvensis (Antir. arvense RE. - Antir Bauhini Gaud. $\beta$ Bertol.) - Nelle vigne e nei campi di Monpantero e di Foresto.

Per. Luglio, Agosto.

Linaiola ginestrina it. - L. à feuilles de gênet fr.

Nel testo Ant. arvense Lin. pianta non appartenente alla nostra Flora. (Vedi Bertoloni, Fl. It., vol. VI, pag. 370 e 371 . e vedi Re, Fl. Tor., vol. I, pag. 365).

L. striata DC. (Antir. monspessulanum RE-LIN.). Nei siti sassosi specialmente presso i torrenti.

Per. Luglio, Agosto.

Linaiola odorosa it. - L. rayée fr.

A Bardonêche presso Millaures.

L. vulgaris Milu. (Antir. linaria Re-LiN.) - Linaria o Osyris Off. - Presso Susa nei campi declivi di Croale. Diuretica.

Per. Giugno, Settembre.

Linaiola gialla it. - L. commune fr.

* Fauce della corolla non completamente chiusa.

I. supina Desf. (Antir. supinum Re-LiN.). - Comune nei siti sabbiosi della Dora presso Susa e ad Exilles, su la fede del chiarissimo Allioni.

Ann. Maggio, Giugno.

Linaiola sdraiata it. - L. couchée fr. 
I. elatine MiLl. (Antir. elatine RE-LIN.). - Abbonda nei campi e specialmente a San Giorgio.

Ann, Giugno, Agosto.

Soldina it. - L. élatine fr.

I. spuria Mrle. (Antir. spurium Re-LiN.) - Elatine mas Off. - Vegeta nei siti medesimi. Farmaco disusato.

Ann. Giugno, Agosto.

Cenciomolle maggiore it. - L. bâtarde fr.

I. minor Dess. (Antir. minus Re-Lix.). - Nei campi.

Ann. Maggio, Settembre.

Antirrino minore it. - L. naine fr.

I. cymbalaria MrLl. (Antir. cymbalaria Re-Lin.).Ovunque su le vecchie mura.

Per. Maggio, Settembre.

Cymballaria it. - L. cymbalaire fr.

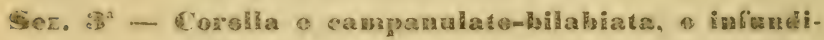
buliforme a rotata-çaadripartita.

GEN. 3. - Digitalim LIN.

(Corolla campanulato-bilabiata)

D. lutea Liv. - Nelle selve. Presso Susa nei siti sassosi della Brunetta.

Per. Giugno, Agosto.

Digitaria it. - Digitale jaune fr.

0. ambigua Lin. Fil. Murr. Syst. - Nelle selve. Velenosa, acre, diuretica.

Per. Giugno, Luglio.

Digitale grande gialla it. - D. à grandes fleurs fr.

(Dig. grandiflora All.). 
Richiamiamo l'attenzione dei medici su l'azione che il Re attribuisce a questa specie di digitale.

\section{Gen. 4. - Gratiola Lin.}

(Corolla infundibuliforme)

G. officinalis LiN. - Gratiola o Gratia Dei Off. Presso i laghi di Avigliana: nei siti umidi di Prato Molle e di Prato Donio presso Susa. Drastica.

Per. Luglio, Agosto. (Fiori bianchi, o debolmente roseogiallicci).

Graziola it. - Gratiole officinale fr.

Usata una volta come purgante drastico; oggi è caduta in dimenticanza.

\section{Gen. 5. - Venomier Lin.}

(Corolla rotata-quadripartita).

* Racemi o spighe ascellari.

* V. prostrata Lin. - Pascoli sterili di Alpignano nel sito detto la Campagnola (Re, Fl. Tor.) - Nella valle d'Oulx (Allioni Ped.).

Per. Aprile, Maggio.

Thè selvatico it. - Véronique couchée fr.

\section{V. teucrium LiN.}

a. normalis Gren e Godr. (V. teucrium Lin. A. Re). - Frequentissima nei siti montani.

$\beta$ latifolia Gren. e Godr. ( $\boldsymbol{V}$. latifolia Re-Lin. - V. feucrium $\beta \mathrm{RE}$ ) - Pascoli montani ed alpestri: lungo la via che conduce alla borgata Le Jambon tra Exilles e Salbertrand.

Per. Aprile, Giugno,

Veronica laneiuola, erba celestina it. - V. teucriette fr. Vedi Colla, Herb., vol. IV, pag. 339. 


\section{V. beccabunga Liv. - Beccabungae o Anagallidis} aquaticae herba Off. - Siti acquosi pingui non lontani dalle fonti. Antiscorbutica.

Per. Maggio, Agosto.

Beccabunga it. - V. beccabonga cressonnière fr.

È mangiata da taluni in insalata; è amaro-tonica.

V. anagallis Lrv. - Veg'eta nei siti medesimi della specie precedente.

Per. Maggio, Agosto.

Crescione, erba grassa it.-- V. mouron : Mouron d'eau fr.

V. chamaedrys LIN. - Chamaedrys folia o Veronica pratensis Off. - Ovunque nelle siepi e nelle selve. Farmaco disusato.

Per. Aprile, Giugno.

Crescione dei prati it. -. V. petit chêne: fausse germandrée fr.

* V. urticaefolia JACQ. - Musinè (Gras) ove la raccogliemmo anche noi.

Per. Maggio, Luglio.

Veronica ortica it. - V. à feuilles d'ortie fr.

V. officinalis LiN. - Frequente nelle selve montane.

Per. Giugno, Luglio.

Veronica maschio it. - V. officinale fr.

V. Allionii Vill. (V. pyrenaica Re-ALL.). - Pascoli: del Cenisio. Sudorifera.

Per. Luglio, Agosto.

Veronica di montagna it. - V. d'Allioni fr. 
Assietta: Alpi di Bussolino alla Balmetta. $\dot{\mathrm{E}}$ a questa specie che quei montanari danno il nome di The di montagna per averla sperimentata sudorifera: rimane ad assodare se il sudore sia l'effetto della Veronica ovvero dell'acqua calda nella quale è presa.

V. aphylla LiN. - Luoghi ombrosi del Cenisio. Per. Giugno, Agosto.

Veronica piccina it. - V. à feuilles radicales fr. V. aphylla Lin. y holocarpa Ces. Comp. Fl. it.

- Racemi o spighe terminali dei cauli e dei rami.

V. spicata LiN. - Pascoli e prati macilenti: abbonda presso Susa nelle rupi a mezzodi di Prato Donio.

Per. Luglio, Agosto.

Veronica spigata it. -- V. à épis fr.

V. bellidioides LIN. - Nei pascoli del Cenisio.

Per. Luglio, Agosto.

Veronica margherita it. - V. pâquerette fr.

V. fruticulosa Lin. - Pascoli sassosi del Cenisio e all'Assietta.

Per. Luglio, Agosto.

Veronica fior di rosa it. - V. ̀̀ souche ligneuse fr.

V. fruticulosa Lin. $\beta$ pilosa Gren. e Godr. (V. saxatilis Jacq.).

V. Alpina Lin. - Pascoli umidi e freddi del Cenisio.

Per. Luglio, Agos to.

Erba gualda alpina it. - V. des Alpes fr. Assietta. 
V. serpyllifolia Lin. - Nei prati umidetti.

Per. Aprile, Giugno.

Erba gualda selvatica it. - V. serpolet fr.

V. arvensis LrN. - Copiosissima negli orti $e$ nei campi.

ß Bellardi (V. Bellardi Re-Alt.). - Trovai questa Veronica, che io sospetto essere varietà di V. arvensis prodotta dalla località secca, nei siti aridi montani di Chianoc, e nelle località secche e calde di Condove.

Ann. Aprile, Maggio.

Ederello it. - V. des champs fr.

V. acinifolia LiN. - Nei campi.

Ann. Aprile, Maggio.

Veronica timo it. - V. à feuilles de thym fr.

V. verna Lin. - Ovunque nei pascoli e nei campi.

Ann. Aprile, Maggio.

Veronica primaticcia it. - V. printanière fr.

V. praecox Alc. - Nella valle di Exilles presso i campi, ma oltremodo rara.

Ann. Marzo, Aprile.

Veronica campagnuola it. - V. précoce fr.

V. triphyllos LiN. - Abbonda nei siti aprici presso Susa.

Ann. Marzo, Maggio.

Veronica manine it. - V. à trois lobes fr, 
* Fiori ascellari solitari.

V. didyma Ten. (V. agrestis Re-ALl.) - Copiosis. sima nei campi e negli orti.

Ann. Marzo, Maggio, spesso in Febbraio.

Gallinella prataiuola it. - V. rustique fr.

Nel testo V. agrestis Lin. dall'Allioni.

* V. Buxhaumi Ten. - Localita della precedente. Ann. Marzo, Aprile.

Veronica querciola it. - V. filiforme fr.

V. hederaefolia Lin. - Frequente nei campi e nei colli.

Ann. Marzo, Maggio.

Veronica morso di gallina it. - V. à feuilles de lierre fir

ORdrne LXXI - ORobancoiden (Orobancheae Jiss.:.

Gen. 1. - Drobanelhe Lin.

- Stami inseriti sotto il terzo inferiore della corolla.

a) Filamenti pelosi.

0. galii Duby (0. caryophyllacea Re-WiLld.). - Da me raccolta su le rupi delle Blaccie.

Per. Maggio.

Succiamele garofanato it.

Località medesima: su le radici del Galium mollugo (Cesati). 
* 0. taucrii F. W. Schultz. - Siti arenosi della Dora presso Caselette: falde della Sagra di San Michele (Gras) su le radici del Teucrium chamaedrys, montanum, scorodonia e del Thymus serpillum.

Per. Giugno.

* 0 . cruenta $\beta$ gracilior Bertol. (0. gracilis Surth.). - A Susa su le radici di varie leguminose, Genista tinctoria, Lotus corniculatus, etc. (Cesati).

Per. Aprile, Maggio. (Corolla scuro-sanguigna, o sanguigna).

Succiamele sanguigno it. - Orobanche couleur de sang fi'.

b) Filamenti glabri per lo meno nella metd inferiore.

Orobanche major Lis. - Ovunque nei prati e nei pascoli macilenti e nelle rupi: presso Susa abbonda su le rupi delle Blaccie e di San Lazzaro.

Per. Giugno, Luglio.

Succiamele maggiore it. - Orobanche rave fr.

(0. rapum Thuill. $y$ braeteosa Reut?).

* Stami inseriti al di sopra del terzo inferiore della corolla.

a) Filanenti del tutto glabri.

* 0. amethystea ThuilL. - Sagra di San Michele su le radici dell'Eringium campestre (Gras): probabilmente altrove nell'ambito della Flora.

Per. Maggio, Giugno.

Succiamele dell'Eringio it. - 0. du panicaut champètre fr. 
b) Filamenti pelosi.

* $\mathbf{0}$. hederae DC. - Su le radici dell'edera nei dintorni di Susa (Cesati).

Per. Aprile, Giugno.

Succiamele minore it. - Orobanche du lierre fr.

\section{Gen. 2. - Phelipaea Desf.}

Ph. caerulea A. Meyer. (Orobanche laevis Re-Lank.). - Nei siti montani aprici tra Foresto e S. Giuliano.

Per. Giugno, Luglio.

Succiamele porporino it. - Phélipée bleuâtre fr.

La località piemontese non è riportata nè dal Bertoloni nè dal Comp. Fl, it. Cesati, etc.

$\mathrm{Nel}$ testo Or. laevis Lin.

Ph. ramosa A. Meyer. (Orobanche pamosa Re-LiN.). - Nei campi spesso su le radici delle piante, e specialmente della Cannabis sativa.

Ann. Agosto, Settembre.

Succiamele piccolo it. - $\mathrm{Ph}$. rameuse fr.

\section{GEN. 3. - Lathraea LIN.}

L. Squamaria LiN. - Siti ombrosi e pingui delle selve: a Susa nei luoghi selvatici della Bretagna, e presso i ruscelli a San Saturnino.

Per. Aprile, Maggio.

Dentaria it. - Lathrée écailleuse fr. 
Ordine LXXII - Rinantacee (Rhinanthaceae DC.)

\section{Gen. 1. - Tozeia Lin.}

T. alpina Lin. - Frequente presso i torrenti al Colle della Russa nelle alpi di Giaveno.

Per. Luglio, Agosto. (Fiori gialli).

Tozzia alpina it. - Tozzie des alpes fr.

Altipiano del Cenisio (Negri).

\section{GEN. 2. - M M ellampyroum LiN.}

Spiga quadrangolare densa.

\$. cristatum LrN. - Siti montani elevati.

Ann. Giugno, Luglio. (Brattee verdastre piegate).

Tritico vaccino crestato it. - Mélampyre à crêtes fi:

- Spiga non quadrangolare poco densa spessissimo unilaterale.

M. arvense Lix. - Comunissimo nelle messi.

Ann. Giugno, Luglio. (Brattec rosse pennatifide).

Coda di volpe: fiamma it. - M. des champs fr.

M. pratense Lrx. - Nelle selve apriche montane. Ann. Giugno, Luglio. (Brattee verdi lanceolate dentate alla base).

Melampiro bianco it. - M. des prés fr.

M. nemorosum Lix. - Nelle selve: abbonda a! Musinè.

Ann. Giugno, Luglio. (Brattee cuoriformi azzurre rar. bianche).

Melampiro azzurro it. - M. des bois fr. 
M. sylvaticum Liv. - Ovunque nelle selve alpine: abbonda nei pineti presso il borgo di Cirrati a Mocchie, e a Cels in valle d'Exilles.

Ann. Giugno, Luglio. (Brattee lanceolate intere o appena dentate alla base).

Melampiro fiorellino it. - M. des forêts fr.

Nelle selve a Bardonêche.

\section{GEN. 3. - Pedicularis Lin.}

- Elmo (labbro superiore della corolla) senza rostro e senza denti.

P. foliosa Lin. Mant. -- Prati pingui del Cenisio presso la Ramasse, nel piccolo monte Colissè e nelle alpi di Bardonêche.

Per. Giugno, Luglio. (Fiori gialli).

Pedicolare fronzuta it. - Pédiculaire à épi feuillé fr.

P. rosea Wulf. (P. hirsula Re-All.-Vill.). - Prati alpini di Monpantero e del Cenisio, e nelle sommità delle alpi di Giaglione e del Vallon.

Per. Luglio, Agosto. (Fiori rosei).

Pedicolare sottile it. - P. rosée fr.

Nel testo P. hirsuta Lin.

P. versicolor Wahlenb. ( $P$. flammea Re-All.). Nel Cenisio su la fede del chiaro Allioni.

Per. Giugno, Luglio. (Corolla zolfina col labbro superiore macchiato di rosso).

Pedicolare macchiata it. - P. variée $f^{2}$.

P. verticillata Lin. - Presso i ruscelli al Collet andando a Fenestrelle, e nei prati e pascoli umidi del Cenisio.

Per. Luglio, Agosto. (Fiori rossi).

Pedicolare a ghirello it. $-P$, verticillée fr. Alpi di Bussolino. 
* Elmo brevemente rostrato: rostro prolungato alla base in due denti acuti.

P. comosa Liv. - Prati alpini di Monpantero e del Cenisio.

Per. Giugno, Luglio. (Corolla giallo-sbiadita). Pedicolare pallida it. - P. à toupet fr.

* P. sylvatica Lin. - Presso Susa (Boisser) Cenisio (Bonjean) dal Comp. Fl. it. Cesati, etc.

Ann. Maggio, Giugno. (Corolla roseo-pallida). Pedicolare selvana it. - P. des bois fr.

P. palustris LrN. - Siti umidi dei prati intorno ai laghi di Avigliana.

Ann. Giugno. (Corolla purpurea).

Pedicolare it. - P. des marais fr.

* * Elmo lungamente o brevemente rostrato : rostro tronco senza denti alla base.

* P. incarnata JACQ. - Cenisio (Bonjean, Balbis in Bertol.).

Per. Giugno, Lugiio. (Corolla carnicino-rosea).

Pedicolare carnicina it. - P. incarnate fr.

* P. atrorubens Schleich. - Cenisio (Bouvier). Ann. Luglio, Agosto. (Corolla rosso-scura). Pedicolare cremisina it. - P. du Saint-Bernard fr.

* P. fuberosa Lrn. - Altipiano del Cenisio. (Negri).

Per. Luglio, Agosto. (Corolla gialla).

Pedicolare zolfina it. - P. tubćreuse fr. 
P. fasciculata BeLt. - Cenisio (Bouvier).

Per. Luglio. (Corolla rosea).

Pedicolare fascicolata it. - P. en faisceau fr.

(P. gyroflexa Gaud).

P. gyroflexa VILL. - Nei prati alpini di Foresto, del Cenisio e di Giaveno.

Per. Luglio, Agosto. (Corolla rosea).

Pedicolare cancalina it. - P. arquée fr.

(P. Bonjeannii Colla. P. Cerisia Bonjean).

P. rostrata LIN. - Sommità del Cenisio.

Per. Luglio, Agosto. (Corolla rosea).

Pedicolare beccuta it. - P. à long bec fr.

\section{GEN. 4. - Mhimamthug LIN.}

* Brattee verdi, le superiori con denti acuminati.

R. minor. Енrн. (R. crista galli Re a. Lin.) - Tartrè vern. - Abbonda nei prati macilenti e nei campi montani.

Ann. Maggio, Giugno.

Cresta di gallo it. - Rhinanthe à petites fleurs fr.

* Brattee scolorite, le superiori con denti cuspidati.

* R. alectorolophus Pollich. -- Località medesime. Cenisio. (Bonjean in Bertol.).

Ann. Giugno, Agosto.

Cresta di gallo pelosa it. - Rh. major fr.

\section{GEN. 5. - Mardsia LiN.}

B. alpina LrN. - Siti freddi ed umidi alpini di Monpantero, del Cenisio e delle Alpi di Giaveno.

Per. Luglio, Agosto.

Clinopodio alpino it. - Bartsie des alpes fr. 


\section{GEN. 6. - Euphreåa LiNK. \\ * Calice villoso-glandoloso.}

\section{E. officinalis Liv. in parte Euphrasiae herba 0 ff.}

- Frequente nei pascoli sterili.

Ann. Maggio, Agosto.

Eufrasia it. - Euphraise officinal fr.

* Calice glabro o pubescente ma non glandoloso.

Euph. minima Schleich. (Euph. officinalis $\beta . R E$ ). Eufrasia alpina piccola a fiori gialli. Tournf. inst. 174. - Cenisio sotto la rupe la Tuva, su la fede del ch. Allioni.

Ann. Luglio, Agosto.

Eufrasia minima it. - E. naine fr.

* Euph. salisburgensis Funr. -- Cenisio (Bonjean in Bertol).

Ann. Giugno, Agosto.

Eufrasia it. - E. de Salzbourg fr.

\section{Gen. 7. - Ddlontites Haller.}

Od. lutea Ster. (Euphrasia lutea Re-Lin.) - Pascoli aridi e campi montani.

Ann. Agosto, Settembre.

Perlina gialla it. - Odontites jaune fr.

Presso Susa. (Bonjean in Bertol).

Od. viscosa Reich. (Euphrasia viscosa Re-Lin. Mant.).

- Raccolta da Ignazio Molineri nei pineti sopra Foresto. Trovasi pure nella valle di Bardonêche.

Ann. Luglio, Agosto.

Peolina appiolina it. - Od. visqueuse fr.

Pineti di Foresto (Molineri in Bertol.). 
Od. serotina Rerch. (Euphrasia odontites Re $\beta$ LIX.)

- Nei campi sabbiosi.

Ann. Luglio, Agosto.

Peolina rossa it. - Od. tardive fr.

Ordine LXXiII. - Labiate (Labiatae Juss).

Sez. - $^{2}$ - Due stami : gli altri due o mancauti o sterihi.

GEN. 1. - Salvia LIN.

(2 stami).

* S. officinalis Lry. - Salvia vern. - Coltivata nei giardini. Essa è origrinaria di siti meridionali di Italia.

Per. Maggio, Giugno.

Salvia it. - Sauge officinale fr.

Un giorno godette di una grande reputazione medicinale.

Cur moriatur homo cui salvia crescit in horto?

Oggi è amministrata in infusione come tonico eccitante, e serve anche per condimento.

S. aethiopis Lin. - Presso i campi ad Oulx, nella valle di Bardonêche, e tra Bussolino e Foresto.

Ann. Maggio, Giugno.

Etiopide it. - S. d'Ethiopie fr.

S. glutinosa Lin. - Frequente nelle selve.

Per. Luglio, Agosto.

Fuso di Giove it. - S. glutineuse fr.

S. pratensis LiN. - In tutti i prati. Subaromatica stimolante.

Per. Maggio, Luglio.

Chiarella it. - Bouras servaj vern. - S. des près fr. 


\section{GEN. 2. -. Rosmanimus TOURnF.}

(2 stami).

* R. officinalis LiN. - Coltivato negli orti per condimento. Originario di siti meridionali marittimi.

Suffruticoso. Maggio, Agosto.

Rosmarino it. - Rosmarin vern. - Romarin officinal fr.

\section{Gen. 3. - Lyeopus LIN.}

(4 stämi, 2 sterili).

L. europaeus LIN. - Abbonda lungo i fossi.

Per. Luglio, Settembre.

Erba sega it. - Lycope d'Europe, lance du Christ fr.

\section{Sez. æ. - Quattro stami tutti fertili.}

A) Corolla all'aspetto unilabiata.

\section{Gen. 4. - Teuerioum LIN.}

* Calice a 5 denti subeguali.

T. hotrys LiN. - Botrys chamaedryoides Off. - Nei campi e lungo la Dora; stimolante.

Ann. Maggio, Luglio. (Fiori porporini).

Camedrio secondo it. - Germandrée botride fr.

T. chamaedrys LiN. - Chamaedryos off. - Calamandrea vern. - Frequentissimo nei pascoli secchi. Amaro, febbrifugo.

Per. Giugno, Luglio. (Fiori porporini).

Camedro it. - G. petit chêne fr.

È rimedio popolare contro le febbri intermittenti; ma realmente non è che un amaro tonico digestivo, come, in minor grado, qualche altra specie di Teucrium notata come medicinale dall'autore. 
T. montanum Lrs. - Abbonda in siti aprici e sassosi, montani, esposti a mezzodi.

Per. Maggio, Agosto. (Fiori bianchi).

Ramerino montano it. -- G. de Montagne fr.

T. scordium Lin. - Scordium Off. - Intorno ai laghi di Avigliana e lungo i fossi nella via a Sant'Antonino. Stomatico : antielmintico.

Per. Luglio, Agosto. (Fiori porporini).

Scordio it. - G. scordium, Chamarras fr.

È superfluo accennare che val nulla come antielmintico.

* * Calice bilabiato : fiori giallicci.

T. scorodonia Lin. - Salvia agrestis o Scorodonia Off. - Frequente specialmente nei castagneti. Abbonda specialmente lungo la via che dalla Chiusa conduce al tempio della Sagra di S. Michele. Amaro.

Per. Giugno, Luglio.

Scorodonia it. - G. taux scordium fr.

\section{GEN. 5. - juga LiN.}

* Fiori solitari, gialli.

A. chamaepitys (Teucrium chamaepitys RE-LiN.) - Ivae artethicae herba Off. - Qua e là nei campi sterili e presso le mura. Amara, stimolante.

Per. Luglio, Settembre.

Artetica it. - Bugle faux pin fr.

* * Fiori agglomerati azzurri, rar. bianchi.

A. piramidalis Lin. - Qua e là nei pascoli.

$\beta$. Bugula alpina maxima Tournf. inst. 209, raccolta, come scrisse il chiaro Allioni, da Fe- 
lice Valle a piede del monte Chapé, presso Chiomonte, e nel monte il Vallon, ed anche tra Cels ed Exilles. Subastringente.

Per. Maggio, Giugno.

Bugola rossa it. - Bugle pyramidale fr.

(Cenisio, Bonjean in Bertol.).

A. genevensis Lin. (Ajuga alpina RE; e All. sotto Bugula.) - Cenisio: alpi di Giaveno.

Per. Maggio, Giugno.

Bugula silvana it. - B. de Genève fr.

Nel testo Ajuga alpina Lin., specie diversa non italiana.

A. reptans Lin. - Bugula, consolida, media, e Symphitum medium Off. - Ovunque nei prati e nei pascoli umidi. Subastringente.

Per. Aprile, Maggio.

Bugola it. - B. rampante fr.

B) Corolla quadriloba non bilabiata.

Gen. 6. - Mendh Lin.

* Fauce del calice nuda.

M. sylvesiris Lin. e Off. - Ovunque lungo i fossi dei prati e nei siti umidi.

Per. Luglio, Agosto.

Menta selvatica it. - Menthe sauvage fr.

M. rotundifolia Lin. - Mentha alba, o Menthasirum Off. - Qua e là nei pascoli. Antispasmodica.

Per. Luglio, Agosto.

Mentastro it. - M. à feuilles rondes fr. 
M. arvensis LiN. - Frequente nei campi freddi. Virtù della precedente:

Per. Luglio, Agosto.

Menta it. - M. des champs fr.

M. aquatica Lis. - Comune nei prati acquosi. Abbonda a Susa dietro la Brunetta. Virtù delle precedenti.

Per. Luglio, Agosto.

Menta d'acqua it. - M. aquatique fr.

* Fauce del calice chiusa da una corona di peli.

M. pulegium Lrs. - M. pulegii herba Off. Lungo la via presso Pietra Cultera, e nei pascoli similmente inondati. Aromatica, antispasmodica.

Per. Luglio, Agosto.

Menta piccola it. - M. pouliot fr.

Le acque medicinali delle varie Mente, sopra notate, sono carminative antispasmodiche.

\section{C) Corolla bilabiata.}

1) Stami posteriori pit lunghi degli anteriori.

\section{Gen. \%. - Nepeta Lin.}

Lobo medio del labbro inferiore della corolla concavo orbicolare.

N. cataria Lin. - Nepeta o Menta cataria Off. Presso le case e nei siti incolti. Antispasmodica.

Per. Luglio, Agosto.

Menta dei gatti it. - Népéta chataire fr.

Fr un giorno adoperata come amara antiscorbutica, antispasmodica, ecc. 亡̀ detta menta dei gatti, perchè quest $i$ animali sono attirati dal suo odore, ed amano avvoltolarsi sa la terra ov'essa cresce. 
N. nepetella LiN. - Siti sassosi in valle di Exilles. Virtù della precedente.

Per. Luglio, Agosto.

Ortica pelosa piccola it. - N. à fleurs lâches fr.

N. nuda LiN. - Nei prati.

Per. Luglio, Agosto.

Mentastro it. - N. nue fr.

* Lobo medio del labbro inferiore della corolla: piano cuoriforme a rovescio.

N. glechoma Bentham. (Glechoma hederacea Re-Lin.). - Hederae terrestris herba 0ff. - Volgarissima nei siti incolti intorno ai campi. Amaricante.

Per. Aprile, Maggio.

Edera terrestre it. - Gléchome lierre terrestre fr.

GEN. 8. - Dracoeephalum LrN.

D. Ruyschiana Lin. - Al di là del piccolo Cenisio nel sito detto Comba d'Ambin, e in cima del Col de la roue verso Bardonêche.

Per. Luglio, Agosto.

Melissa prunellina it. - Dracocéphale de Ruysch fr.

2) Stami anteriori pit lunghi dei posteriori.

A) Stami piegati sul labbro inferiore della corolla GEN. 9. - Lavandula LIN.

L. spica $\alpha$ Lrn. - Lavandula o Spica nardi Off. Lavanda vern. - Abbonda nelle rupi presso Susa, e in tutta la valle di Exilles e di Cesana. Aromatica, antispasmodica.

Per. Luglio, Agosto.

Lavanda it. - Lavande spic fr.

Ha odore soavissimo a tutti noto: ed è adoperata \più per profumeria che come farmaco. 


\section{* Gen. 10. - Deymum Lin.}

0. basilicum Lin. - Coltivato per odore e condimento. Originario delle Indie.

Ann. Luglio, Agosto.

Basilico it. - Basilicò vern. - Basilic fr.

B) Stami convergenti alla sommità.

GEN. 11. - Satureja Lin.

S. montana Lis. - Pascoli sassosi montani : aromatica, antispasmodica.

Per. Luglio, Agosto.

Santoreggia it. - Sarriette de montagne fr.

\section{Gen. 12. - Calamintha Bentham.}

* Tubo del calice curvo.

C. acinos Clatrv. in GAUd. (Thymus acinos Re-LiN.). - Volgare nei campi: alle Blaccie presso Susa.

Per. Aprile, Agosto.

Timo salvatico it. - Calament acinos fr.

c. alpina Lamk. (Thymus alpinus Re-LiN.) - Abbonda nei pascoli alpini.

Per. Maggio, Agosto.

Acino odoroso it. - C. des alpes fr.

Musinè (Gras).

C. Glinopodium Benth. (Clinopodium vulgare RE-LIN.)

- Ovunque nei pascoli e nelle siepi.

Per. Luglio, Agosto.

Menta senza odore it. - C. clinopode fr. 
* * Tubo del calice diritto.

C. grandiflora MoEnch. (Melissa grandiflora RE-Lin.). - Ovunque nelle selve montane. Antispasmodica. Per. Luglio, Agosto.

Melissa peperina it. - C. à grandes fleurs fr.

C. parviflora Lamk. (Melissa Nepeta Re-Lin.) - Volgarissima nei campi. Antispasmodica.

Per. Luglio, Settembre.

Nepitella it. - Petit calament fr.

C. officinalis Moench. (Melissa Calamintha Re-LiN.) - Calaminthae montanae herba Off. - Ovunque nelle selve.

$\beta$ Melissa petiolis ex foliorum alis longissimis et repetito ramosis Haller Helv., 652; trovata, come si rileva dalla Fl. Pedem. del ch. Allioni, da F. Valle alle falde del monte Crevasses in valle di Exilles. Subaromatica.

Per. Giugno, Settembre.

Calamento it. - C. officinal fr.

\section{GEN. 13. - Meliss Lin.}

M. officinalis LIN. - Melissa, Citronella, Citraria Off. - Melissa vern. - Nei siti incolti. Antispasmodica. Per. Luglio, Agosto.

Melissa it. - Mélisse officinale fr.

La Melissa è carminativa. ì a tutti nota la fragranza delle sue foglie. 

C) Stami divergenti alla sommità.

GEN. 14. - Drigam Lin.

\section{0. vulgare Lrv. - Origani herba Off. - Cornabusa} vern. - Abbonda lung’o le vie e le siepi, e nei declivi sassosi. Antispasmodica.

Per. Luglio, Agosto.

Origano it. - Origan commun fr.

\section{Gen. 15. - Thyous Lin.}

T. serpillum Lin. - Serpilli herba off. - Serpol vern. - Frequentissimo nei pascoli sterili e declivi. Aromatico, antispasmodico.

Per. Maggio, Agosto. (Peli, quando esistono, ricurvi).

Timo it. - Thym serpolet fr.

Questa specie è proteiforme e, come osserva il Bertoloni. divisa e suddivisa dai botanici in numerose specie e varieta. Parecchie forme incontri nei monti della nostra fiora, c tra le altre più spiccata Thymus serpyllum $\beta$ Bertol., (Thym. montanus Waldst e Kit).

T. pannonicus AlL. - Siti sassosi alla Brunetta, e sabbiosi alla Dora presso Exilles.

Per. Luglio, Agosto. (Irsuto: peli patenti).

Pepolino peloso it. - Thym de Pannonie fr.

Cenisio al Mollaret (Bouvierr. Alla Novalesa (Bonjean in Bertol.).

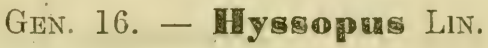

4. officinalis Lrn. - Hyssopus Off. - Nella valle di Bardonêche. Leggriero stimolante.

Per. Luglio, Agosto.

Issopo it. - Hyssope officinal fr. 
L'issopo è astringente, eccitante, e può giovare nei catarri viscerali e bronchiali (Cantani).

Stami paralleli.

A) Calice nè bilabiato nè gonfo.

Gen. 17. - Lamionm LiN.

* Antere glabre.

L. Galeobdolon Crantz. (Galeopsis galsobdiolon RE-Lin). - Ovunque nelle selve montane ombrose.

Per. Maggio, Luglio.

Ortica gialla it. - Lamier galeobdolon fr.

* * Antere barbate.

L. maculatum Lin. - Miizatella Off. - Nelle siepi. Farmaco disusato.

Per. Aprile, Agosto.

Dolcimela it. - L. taché fr.

L. purpureum Lrs. o Rubrum Off. - Abbonda negli orti e nei siti pingui. Farmaco disusato.

Ann. Marzo, Settembre.

Ortica morta it. - Urtie morte vern. - L. pourpre fr.

L. amplexicaule Lrn. - Frequente nei campi e nei ruderati.

Ann. Marzo, Settembre.

Erba ruota it. - L. embrassant fr.

Gen. 18. - Leomerams Lin.

L. cardiaca Lin. - Cardiaca Off. - Frequente presso le case nei siti pingui. Amaro.

Per. Luglio, Agosto.

Cardiaca it. - Agripaume cardiaque fr. 
Gen. 19. - Galleopsis Lin.

G. Iadanum Lrs. - Abbonda nei campi sabbiosi

e nei pascoli.

Ann. Luglio, Agosto.

Gallinella it. - Galéope des champs fr.

G. tetrahit LIN. - Ovinque nelle selve cedue e presso i fossi in luoghi pingui.

Ann. Luglio, Agosto.

Canape selvatica it. - G. tetrahit $\mathrm{fr}$.

\section{GEn. 20. - - Mtatays Lin.}

(Stami prima della fecondazione paralleli, dopo contorti e curvi al di fueri).

* Fiori gialli.

St. annua LiN. - Campi montani. Astringente.

Ann. Luglio, Agosto.

Erba strega it. - Épiaire annuelle fr.

St. recta Lin. - Sideritis o Herba judaica o Totrahit Dff. - Ovunque in siti sassosi e declivi sterili. Astringente.

Stringonella it. - E. redressée fr.

* Fiori porporini.

St. sylvatica Lrv. - Galeopsis o Urtica iners magna footidissima 0ff. - Intorno alle selve. Farmaco disusato.

Per. Luglio, Agosto.

Matricale it. - E. des bois fr.

St. palustris LiN. - Siti umidi di S. Antonino.

Per. Luglio, Agosto.

Scabbiosa it. - E. des marais fr. 
Si. alpina Lin. - Intorno alle selve a Fossimagna.

Per. Luglio, Agosto.

Salvia alpina it. - E. des alpes $\mathrm{fr}$.

St. germanica Lin. - Pascoli e siti incolti.

Per. Giugno, Agosto.

Erba San Pietro it. - E. d'Allemagne fr.

\section{Gen. 21. - Redomica Lin.}

B. officinalis Lis. - Betonica Off. - Frequente nei pascoli montani ed alpini. Eccitante? Errina?

Per. Giugno, Agosto.

Betonica it. - Bétoine officinale fr.

La radice della betonica è amam, e talmi la consideInrono come tonica, altri come emetica e purgativa. Eecu quanto è rimasto delle numerose virtù attribuite dagli anticlii a questa specie, della quale non si fa più uso nella farmacia recente.

B. hirsuta Lin. - Nei prati e nei pascoli del Cenisio. Virtù della prècedente.

Per. Luglio, Agosto.

Betonica porporina it. - B. herissée fr.

(B. Monierii Gouan. All.).

Quali virtù se l'autore le rnette, e con ragione, in dubbio?

\section{GEN. 22. - EBallota Lin.}

B. nigra Lin. - Marrubium nigrum o Ballota off.

- Frequente nei ruderati. Farmaco disusato.

Per. Giugno, Agosto.

Ballota it. - Ballote fétide, Marrube noir fr. 


\section{GEN. 23. - Darabium LIN.}

M. vulgare Lin. - Marrubium, Prasium o Marrubium album Off. - Marrubi vern. - Ovunque nei ruderati presso Susa. Subamaro febbrifugo.

Marrubio it. - Marrube commun fr.

Tenne un grido di gran rimedio nella tisi, e in generale s.elle malattie polmonari; oggi è poco o niente usato.
B) Calice bilabiato gonfio.
GEN. 24.
- Melliteis LiN.

M. Melissophyllum LiN. - Melissa sylvestris o Melissophyllum Off. - Nelle selve montane. Leggermente aromatico.

Per. Luglio, Agosto.

Melino, bocca di lupo it. - Mélitte à feuilles de mólisse fr. Dà un odore leggero ma soavissimo.

C) Calice bilabiato non gonfio.

GEN. 23. - Seatellaria LIN.

(Dente superiore iel caliee a forma di squama concava (scotella).

* Spighe terminali.

S. alpina Lriv. - Pascoli del Cenisio.

Per. Luglio, Agosto.

Scutellaria alpina it. - Toque des alpes fr.

* * Fiori ascellari.

S. galericulata Lin. - Tertianaria Off. - Presso i laghi di Avigliana, e siti umidi presso Giaveno. Amara.

Per. Luglio, Agosto.

Terzianaria it. - T. casside fr.

Usata un giorno nelle febluri intermittenti terzane leggiere. 
* S. minor Lin. - Intorno a! laghhi di svigliara. (Gras).

Per. Giugno, Agosto.

Scutellaria sanguigna it. - $\mathrm{T}$. des marais $\mathrm{fr}$.

\section{Gen. 26. - Bandera Lin.}

B. vulgaris Lin. - Prunella, Brumella, Consolida minor o Symphitum minus Off. - In tutti i pascoli e nei prati umidetti. Subastringente.

y pinnatifida Kocn. (Brunella laciniata RE-LIN.). Frequente nei pascoli secchi.

Per. Luglio, Agosto.

Prunella it. - Brunelle commune fr.

Farmaco disusato.

Ordine LXXIV - Verbenacee (Verbenacene Juss.). Gen. Verbena LiN.

V. officinalis Lin. - Verbenae herba Off. - Barbena vern. - Non rara nei pascoli e nei ruderati. Ann. Giugno, Agosto.

Verbena it. - Verveine officinale fr.

Antico farmaco amaro astringente, oggri smesso. 


\section{ORdine LXXV - Lentibularie}

\section{(Lentibutarieae Juss.)}

\section{GEN. 1. - Pingen benda Lin.}

P. vulgaris Lrs. - Pinguicula Off. - Siti montani ed alpestri: abbonda nei prati umidi ri Coldemozzo. Vulneraria.

Per. Maggio, Luglio. (Fiori violetti. Sperone lineare).

Erba da taglio porporina it. - Grassette commune fr.

: gypsophila Bouvier. Caule più corto, fiori della met‘ più piccoli). Cenisio, (Bouvier). Musinè verso Brione (Grac).

Rimedio degli alpigiani contro le ferite negli unmini, e le screpolature delle mammelle nelle vacche.

P. alpina Lin. - Siti umidi delle aiph. Frequente presso il lago del Cenisio. Trovasi pure a Susa alle Gorgie.

Per. Aprile, Maggio. (Fiore bianco con due macchie gialle alla base del lobo medio del labbro inferiore, sperone conico).

Erba da taglio di fior bianco it. - G. des alpes fr.

Alpi della Novalesa a Brigard (Signora Chiapusso-Voli).

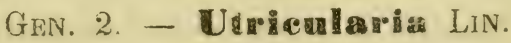

U. vulgaris Lin. - Nelle acque stagnanti.

Per. Giugno, Agosto.

Erba vescica it. - Utriculaire commune fr.

Tanto la Pinguicula che l'Utricularia sono tenute per insettivore dal Darwin, e da altri distinti cultori di fisiologia vegetale. (Vedi Darwin, Le piante insettivore, traduzione di Canestrini e Saccardo. Unione Tip. Torinese, 1878). 
Un curioso fatto di greografia botanica è l'esistenza dellu Pinguicula alpina nella valletta detta Rivauta (m. 500 circa sul mare) tra Pecetto e l'Eremo nei colli di Torino. Fu ivi rinvenuta dal Balbis e dal Re, e noi l'abbiamo raccolta precisamente nel sito indicato da questi autori.

\section{Ordine LXXVI - Primulacee (Primulacene Tent.)}

A) Piante cll"aspetto acauli : fiori su pedunouli radicali.

\section{GEN. 1. - Poimen LIN.}

\section{" Calice angolnso.}

P. Officinalis JACQ. 3 suaveoleus Gren e Godr. (Primula veris Re o. LIx.). - Primula voris flores Dff. Primavere vern. - Intorno alle selve e nei prati. Fiori eccitanti..

Per. Marzo, Aprile. (Fiori gialli: lembo della cornils concavo).

Primavera odorosa it. -- P. officinale fr.

Oggi pressochè disusata in farmacia.

P. grandifiora Lam. (Primula acaulis Re-Alz.) Nei prati. Virtù della precedente.

Per. Marzo, Aprile. (Fiori gialli: lembo della corrlli. quasi piano).

Fior di primavera it. - Primèvere commune fr.

Varia a peduncolo con uno o più fiori.

P. farinosa Liv. -- Pascoli e prati montani er? alpestri. Abbonda presso i Durant nei monti di San Giorgio.

Per. Luglio, Agosto. (Fiori rosei).

Primavera impolverata it. - P. farineuse fr.

Cenisio, cima d'Eau blanche (Negri). 


\section{* Calice cilindrico.}

P. hirsuta AlL - Nel Cenisio e nei monti di Giaveno.

Per. Giugno, Luglio. (Fiori porporini).

Orecchia d'orso pelosa it. - P. visqueuse fr.

Cima d'Eau blanche al Cenisio (Negri).

(Primula viscosa Vill. P. villosa Jacq.).

* P. Iatifolia Lapey (Primula viscosa All. P. villosa $\approx$ Bertol.). - Nelle rupi del piccolo Cenisio (D'Unmbert in Colla Herb., vol. VII, pag. 422 ). Musinè verso il villaggio di Brione (Gras). Colle delle Finestre.

$\beta$ pedemontana (Primula pedemontana Thomas:Cenisio (Bouvier). (Verlot.).

Per. Giugno, Luglio. (Fiori violacei). Orecchia d'orso pelosa it. - P. a grandes feuilles th.

\section{Gen. 2. - Gregoria Dubr.}

G. Vitaliana Duby (Aretia Vitaliana Re-Lix, Srst . - Frequente nei pascoli del Cenisio.

Per. Giugno, Luglio. (Fiore giallo).

Sedo alpino primofiore it. -- Arétie do Vitaliani fr.

Cenisio a Ronche (Negri). Sornmità del colle delle Finestre nei siti umidi.

\section{Gen. 3. - Corthusa Lin.}

(Fiori rosoi: foglie lobate dentate).

C. mathioli Lin. - Presso l'Assietta ad Argeuil, ed anche copiosamente da me raccolta insieme 
ad Ignazio Molineri nel rivo di Codizar sopra Chiomonte.

Per. Maggio, Giugno.

Cortusa it. - Cortuse de Matthiole fr.

Piano del Cenisio (Negri). Cenisio a Savalin (Bouvier).

\section{Gen. 4. - Soldanella LiN.}

S. alpina Lin. - Pascoli ombrosi del Cenisio presso la Fabbrica del marmo.

Per. Maggio, Luglio. (Corolla violacea a lembo laciniato:

Soldanella alpina it. - Soldanclle des alpes fr.

Altipiano del Cenisio (Negri). Colle delle Finestre, della Portia.

Spesso nelle località medesime ineontri una forma minima di questa specie, Soldanella minina Hoppe.

\section{Gen. 5. - Cyelamen LIN.}

C. europaeum LiN. - Cyclamen, Arhanita, Panis porcinus Off. - Occorse al ch. Allioni tra Avighliana e Giaveno. Radice drastrica, catartica e risolvente.

Per. Luglio, Agosto.

Ciclamino pan porcino it. - Cyclane d'Europe fr.

La radice tuberosa del Ciclamino è drastica e purgativit, ma disusata in farmacia.

\section{Gen. 6. - mallosace Lin.}

" Fiuri solitari all'apice dei rami.

A. aipina Lamk. (Aretia alpina RE-Jace.). - Sommità ventose del Cenisio sopra la posta, e nel situ detto Eau blanche.

Per. Laglio, Agosto. 
Sedo alpino stellato it. - Androsace des glaciers fr. Cenisio ad Eau blanche (Negri, Bouvier).

(Androsace glacialis Hoppe).

Nel testo Aretia alpina Lin.

Fiori in ombrella all'apice di uno scapo provvisto d'invoglio a molte foglioline.

A. carnea Lis. - Uvunque nei paseoli del Cenisio. Per. Luglio, Agosto.

Gelsomino di montagna carnicino it. - A. carnée fr.

A. villosa Lin. - In gran copia sopra il boseo nero, eà all'Assietta.

Per. Luglio, Agosto.

Androsace barbata it. - A. velue fr.

Nel bosco nero (Re in Bertol.).

La localiti piemontese manea nel Compendio della Fl. it. del Cesati, etc.

14. oblusifolia Lin. - Ovunque nel Cenisio, e nei luoghi nevosi del Vallon.

Per. Luglio, Agosto.

Gelsomino di montagna it. - A. à feuilles obtuses fr.

A Ronche (Neyri!.

A. chamaejasmo líost. - Cenisio (Compendio della Fl. it., Cesati, etc.).

Per. Luglio, Agosto.

Androsace cigliata it. - A. ciliée fr.

A. maxima Lin. - Campi caldi.

Ann. Maggio, Giugno.

Androsace dei campi it. - A. à grand calice fr. 
282

Nella valle di Exilles in gran copia. Allioni Auct. pag. 6. A Bardonêche, e in abbondanza nei campi di segale ad Oulx. (De Filippi).

L: localita piemontese manca nel Compendio della Fl. it. del Cosati. etc. : secondo i chiari autori vegeterebbe in Taltellina.

\section{B) Piante con caule.}

GEN. 7. - a amoles LiN.

3. Valerandi Lin - Samolus Off. - Volgarissimo lungo i fossi.

Pur. Giugno, Agoste. (Fiori piccoli, bianchi in pannocchia).

Samolo, lino dacpua it. - Samole de Valerandus, Monron d'eall fr.

\section{GLN. 8. - magallis LIN.}

5. arronsis Lix. (Anagallis phoenicea RE-ALL.). Anagallis Mas off. - Nei campi pingui e negli orti. Acre.

F caerulea (Anagallis coerulea Re-AlL.) - Anagallis foemine off. - Volorere nei campi.

Ann. Giugno. Settembre. Fiori color di minio nella specie, azzurri nelle varietà).

Erba grisettina it. - Mouron des champs fr.

Aloperate un giomo come purgante : oggi fuori nen.

\section{CHE. 9. - Ly}

- Fiori in pannocchia terminale.

L. vulgaris Lix. - Lysimachia lutea Off. - Frequente lungo i fossi e presso i siti acquosi. Farmaco disusato.

Per. Giugno, Luglio.

Lisimachia it. - Lysimaque commune fr. 
* Fiori solitari ascellari.

L. nemorum Lin. - Nelle selve alpestri.

Per. Luglio, Agosto.

Centonchio giallo it. - L. des bois 'fr.

L. nummularia Lin. - Nummularia o Centimorbia Off. - Qua e la nei pascoli umidi. Subastringente.

Per. Giugno, Luglio.

Nummularia it. - L. nummulaire, herbe aux écus fir.

$\dot{\mathrm{E}}$ astringente, ma poco o niente usata.

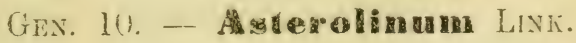

A. Stellatum LiNk. (Lysimachia linum-siellatum KeLis.). - Abbonda nelle vigrne di Monpantero e delle Blaccie juresso Sust, come nei siti decliri di Condove alla Roccèa.

Ann. Giugno.

Lino stellato it. - Asteroline étoilé fr.

Su la Brunetta (Negri).

La località segusina, o meglio addirittura la piemontese, manca nel Compendio della Fl. it. del Cesati, ete. come manca nel Bertoloni Fl. it.

Ordine LXXVII - Globlqahiache (Globularieae DC.)

\section{GEN. Giobularia LIN.}

G. vulgaris Lix. - Frequente nei siti arenosi presso la Dora, e nelle rupi di Condove.

Per. Maggio, Giugno.

Morine it. - Globulaire commune fr. 
G. cordifolia Lin. - Abbouda nelle rupi presso Susa.

Per. Maggio, Luglio.

Vedovella celeste it. - G. à feuilles en coeur fr.

Alle Blaccie e a Gravere (Negri).

* G. nudicatils LIN. - Cenisio (Bonjean in Bertol.)

Per. Giugno, Agosto.

Margherita azzurra it. - C. à tige nue fr.

\section{Ordine LXXVIII - Plumbaginee}

(Plumbagineale Juss.)

\section{Gen. Aramerara WILLD.}

A. alpina Wrutd. (Statice armeria Re-LiN. Fl. suec.) - Piecolo Cenisio.

Per. Luglio, Agosto.

Brillantini lisei it. - Armérie des alpes fr.

Lungo le vie alpestri che dal colle dolle Finestre mentun all'Assietta: monti di Bussulino alla Balmetta superiore. (Statice alpina Hoppe, Statice armeria y alpina DC. Fl. ir. St. vulgaris Bertol, esclusa V. $\beta$. .)

A. plantaryinea Willn. (Statice pseutoarmaria ReMuriz. Srst., Virmans Sum. pl.). - Alle falde del Musinè nei pascoli di Caselette: e andando a Fenestrelle nel sito detto il Collet.

Per. Maggio, Luglio.

Statice a foglie di piantaggine it. $-\lambda$. a feuilles de plantain fr.

Statica plantaginea All. St. Scorzoneraefolia Re (Fl. Tor.)

Nel testo S. pseudoarmeria Lin. 


\section{Ordine LXXIX - Piantaginee}

(Piantagineae Juss.)

\section{GEN. Pantago Lin.}

* Piante acauli : pedunculi radicali.

a) Tubo della corolla glabro.

P. major Lrn. - Plantago minor o Septinervia Off. - Piantai vern. - Volgare intorno alle case e lungo le vie. Astringente.

Per. Aprile, Luglio.

Piantaggine maggiore it. - Plantain a grandes feuilles fr. $\beta$ intermedia (Pl. internedia Gilib.) con la specie.

P. media Lin. (Plantago media o Quinquenervia off.). - Ovunque nei pascoli e nei prati macilenti. Virtù della precedente.

Per. Maggio, Giugno.

Piantaggine mezzana it. - P. moyenne fr.

P. Ianceolata Lin. - Plantago major, o Trinervia, o Plantago mas Off. - Vegeta nei siti medesimi. Virtù delle precedenti.

Per. Maggio, Luglio.

Lanciolo it. - Lenga d'can, fojola vern. - P. lancéolé fr.

P. montana Lamk. (P. alpina Re-Vrrt.). - Siti montani ed alpestri.

Per. Luglio, Agosto.

Piantaggine nera it. - P. de montagne fr.

Cenisio (Balbis, Bonjean in Bertol.). 
Nel testo Pl. alpina Lin. la quale secondo il Cesati nor sareble che una forma svedese della Pl. maritima Lin. (Comp. della Fl. it. del Cesati, pag. 284).

Le piantaggini notate dal Re come medicinali oggi sono raramente adoperate (infusione delle foglie) nei collirii : nelle uretriti come astringenti.

\section{* Tubo della corolla pubescente.}

* P. serpenina All. - Comba di Meana vi:a del colle delle Finestre. Sagra di San Michele.

Per. Giugno, Luglio.

Serpentina it. - Erba dij mouton fr. - P. serpentine fr.

I'. maritima Lin. P Serpentina (Comp. rella Fl. it. Cesati, etc.). P. serpentina All.

- Piante con cauli ramosi.

P. ramosa Asch. (P. psyllium Re-All. non LIN. sec. Bizrtor. - P. arenaria Valdst e Kit.). - Psyllii semen Off. - Nei siti sabbiosi intorno Susa. Semi mucilaginosi.

Ann. Maggio, Luglio.

Psillo it. - P. pucier fr.

P. cynops Lin. - Ovunque nei pascoli montani.

Per. Maggio, Agosto.

Pulicaria legnosa it. - P. des chiens fr. 


\section{DIVISIONE IV.}

Monociamidee (Monochlemydeae).

Ordine LXXX - Ayarantaces:

(Amaranthuceae R. Brovon.)

\section{Gen. 1. - Amaramtag Lin.}

A. blitum Lis. - Campi feraci ed orti.

Ann. Luglio, Agosto.

Blito minore it. - Amarante blette fr.

A. viridis Lin. - Luoghi medesimi.

Ann. Luglio, Agosto.

Blito maggiore it. - A, verte fr.

\section{GEN. 2. - Folyememame LIN.}

P. arvense LiN. - Campi e vigne.

Ann. Luglio, Agosto.

Canforata bastarda it. - Polycneme des champs fr.

\section{Ordine LXXXI - Chenopodiacee}

(Chenopoderue Vent.)

\section{GEN. 1. - M Iid a}

B. bonus Henricus Reich. (Chenopodium bonus henricus Re-LiN.). - Bonus Henricus o Tola bona Off. - Ovunque nei siti pingui alpini. Emolliente.

Per. Giugno, Agosto.

Spinacio selvatico it. - Spinas servai vern. - Ansérine Bon-Henri, Toute bonne fr. 
- Le foglie sono un cibo comune ai pastori delle alpi e del medio appennino ed hanno azione leggermente purgativa. L'origine del nome Buono Enrico è sconosciuta: il Poiret sospetta che sia religiosa. Certo nei mezzi tempi la pianta godette di una grande riputazione medicinale.

B. rubrum Lin. (Chenopodium rubrum Re-Lin.). Nei siti incolti e presso le mura.

Ann. Luglio, Agosto.

Atreplice salvatica it. - A. rouggeâtre fr.

Tanto il Bertoloni (Fl. it.) quanto il Compendio delli: Fl. italiana del Cesati, ete. non indicano localita piemontesi di questa specie. B. rubrum Lin., secondo quegli autori. vegeterebbe nel Mantovano, nel Veneto, presso Pisa ed in Corsica.

\section{GEN. 2. - Chenopodium A. Meyer.}

* Foglie intere.

C. polyspermum Lin. - Campi ed orti.

Ann. Luglio, Agosto. (Pianta inodora).

Bieta grappolosa it. - Ansérine polysperme fr.

C. olidum Smrt. (Chenopodium vulvaria RE-I LIN.).

- Frequente presso le mura.

Ann. Luglio, Agosto. (Pianta fetidissima).

Erba puzzolona, vulvaria it. - A. puante fr.

* Foglie, per lo meno le inferiori, dentate, sinuate o partite.

C. hybridum Lin. - Nei ruderati e presso lo case.

Ann. Luglio, Agosto.

Spinace velenoso it. - A. bâtarde fr. 
C. urbicum LiN. - Nei ruderati e presso le case. Ann. Luglio, Agosto.

Piede di anatra it. - A. des villages fr.

C. murale Lix. - Località medesime.

Per. Maggio, Agosto.

Piede anserino it. - A. des murs fr.

C. Botrys Lix. - Botrys vulgaris herba Off. Qua e là nei siti sabbiosi lungo la Dora e copiosissimo presso il torrente del Gravi nel territorio di Condove. Antispasmodico.

Ann. Luglio, Agosto.

Botri it. - A. botride fr.

Sino a pochi anni addietro era tenuto come calmante nelle tossi tisiche, e convulsive : oggi è farmaco abbandonato.

C. album Lis. - Volgare nei campi feraci.

$\beta$ viride (C. viride Re-Lin.). - Frequente nei siti colti e nei ruderati.

Ann. Luglio, Agosto.

Chenopodio bianco it. - Farinet vern. - A. blanche fr.

C. glaucum Liv. - Nei campi.

Ann. Luglio, Agosto.

Chenopodio azzurrognolo it. - A. glauque fr'

\section{Gen. 3. - triplex LiN.}

A. patula Lin. - Presso le case e negli orti. Ann. Luglio, Agosto.

Atriplice triangolare it. - Arroche étalée fr. 
* GeN. 4. - Spimacia LIN.

Sp. oleracea Liv. - Coltivata per alimento: di origine incerta.

Ann. Bienn. Maggio, Giugno.

Spinaci it. - Spinass vern. - Épinard fr.

Gen. 5. - Theligomum Lin.

T. cynocrambe Lin. - Nelle vigne presso Susa. Ann. Giugno.

Connina it. - Théligone étalé fr.

Ordine LXXXII - Poligonacee (Poligoneae Juss.) GEN. 1. - Mamex LiN.

-Foglie cuoriformio assottigliate alla base, ma giammai astate o sagittate.

R. alpinus LiN. - Rhabarbarum monachorum Off. - Nei siti pingui intorno alle case su le alpi. Radice amaro-tonica, astringente.

Per. Luglio, Agosto.

Rabarbaro alpino it. - Rumex des alpes, Oseille des alpes fr.

La radice era usata una volta invece del rabarbaro: oggi è fuori uso: come pressochè dimenticate in farmacia sono le specie seguenti contenenti più o meno acido ossalico.

R. conglomeratus Murr. (Rumex acutus RE DC. Fl. Fr.) - Lapathum acutum o Oxilapathum Off. - Lungo i fossi. Subastringente.

Per. Luglio, Agosto.

Romice campestre it. - R. à feuilles aiguës fr.

Nel testo R. acutus Lin. 
R. Friesii Gr. e Godr. (Rumex obtusifolius RE DC. Fl. Fr. ). - Siti pingui ed incolti presso le case, e nei prati e nei campi fertili : virtù del precedente.

Per. Luglio, Agosto.

Romice dei prati it. - $R$. à feuilles obtuses fr.

Nel testo Rumex obtusifolius Lin.

R. pulcher Lis. - Nei pascoli ovunque. Virtù del precedente.

Per. Maggio, Giugno.

Romice selvatica it. $-R$. violon $f r$.

- Foglie astate o sagittate.

R. scutatus Lin. - Frequente presso i torrenti e i siti sassosi.

Per. Maggio, Luglio.

Acetosa tonda it. - $R$, à écussons fr.

R. arifolius ALL. - Nelle selve e nei luoghi pingui delle alpi.

Gicchero di montagna it. - $\mathrm{R}$. à feuilles de gonet fr. Vicinanze del lago del Cenisio (Negri).

Nel testo R. arifolius Willd.

R. acetosa Lrn. - Asivola vern. - Ovunque nei prati.

Per: Giugno, Luglio.

Erba brusca selvatica it. - R. oseille fr.

R. acetosella LrN. - Nei campi.

Per. Maggio, Luglio.

Acetosa minore it. $-R$. petite oseille fr. 


\section{GeN. 2. - Oxyria Hill.}

0. digyna Campder. (Rumex digynus Re-Lin.). - Siti freddi del Cenisio.

Per. Luglio, Agosto.

Acetosa soldanella it. - Oxyria à deux stigmates $\mathrm{fr}$. Assietta.

\section{Gex. 3. - Polygonum Lin.}

- Caule semplice terminato da rina spiga unica.

P. bistorta Lis. - Bisiorta major Off. - Abbonda nei prati pingui montani e alpini umidetti. Radice stittica, astringente.

Per. Giugno, Luglio.

Bistorta it. - Renouée bistorte fr.

P. viviparum Lrs. -- Frequente nelle alpi. Virtu del precedente.

Per. Giugno, Luglio.

Bistorta minore it. - $R$. vivipare $\mathrm{fr}$.

Le radici di queste due specie contengono acido tannico e sono astringenti.

* Caule ramificato : rami terminati da una spiga.

P. ampihibium Lin. - Nel laghetto ad occidente della Brunetta, e nei laghi di Avigliana.

Per. Giugno, Luglio.

Persicaria anfibia it. - R. amphibie fr.

$\dot{\mathrm{E}}$ acquatico ( $\%$ natans Moench) e terrestre ( $\beta$ terrestris Moench.). 
P. persicaria Lrn. - Persicaria mitis 0ff. - Intorno agli orti e nei ruderati. Farmaco disusato.

Ann. Luglio, Settembre.

Persicaria it. - Erba roussa vern. - R. persicaire fr.

P. hydropiper Lin. - Persicaria urens o Hydropiper Off. - Orunque lungo i fossi. Acre, diuretico, epispastico.

Ànn. Luglio, Settembre.

Pepe d'acqua it. - Brusarola vern. - R. poivre d'ean fr. Disusato in farmacia.

Caule ramificato:

fiori in racemi su rami formanti una pannocchia terminale.

*P. alpinum Alt. - Cenisio (Bonjean in Bertol.). Per. Luglio, Agosto.

Persicaria alpina it. - Clusion vern. - $R$. des alpes tit. .. Fiori ascellari fascicolati.

P. aviculare Lin. - Polygonum o Centinodia Off. - Erba rabloira vern. - Frequente nelle vie e nei campi. Substittico.

Ann. Luglio, Settembre.

Centinodia it. -- Erba dij crin altro vern. - R. des petits oiseaux fr.

Disusato in farmacia.

P. convolvulus Lis. - Qua e là nei campi.

Ann. Luglio, Settembre. (Foglie cordato-sagittate). Convolvolo nero it. - Curiolon vern. - R. liseron fr. 
Ondine LXXXIII. -- Timeleacee (Thymelene Juss).

\section{Gen. 1. - Passerima LIN.}

P. annua Spreng. (Stellera passerina Re-Lin.). Abbonda nei campi aridi soleggiati presso Susa. Ann. Luglio, Agosto.

Linaria it. - Passerine annuelle fr.

\section{Gen. 2. - Daphme LiN.}

" Fiori laterali o ascellari.

D. mezereum Lis. - Laureola foemina, Thymaelea o Mezereon off. - Siti montani, più frequentemente nelle alpi. La corteccia della radice è velenosa, drastica, emetica; applicata esternamente epispastica, corrodente.

Frutice. Aprile, Maggio. (Fior: rosei fragrantij.

Mezzèreo it. - Dafné mézéréon fr.

Il Mezzèreo è senza dubbio velenoso, e riesce epispastico.

Il suo uso interno ed esterno è oggi molto limitato.

D. Aaureola Lis. - Laureola mas, Laureola Daphnoides Off. - Nelle selve delle blaccie presso Susa. Virtù della precedente.

Frutice. Aprile, Maggio. (Fiori verdi: foglie persistenti). Laureola it. - D. lauréole fr.

La Laureola è velenosa al pari del Mezzèreo.

* Fiori terminali.

* D. alpina Lis. - Cenisio (Bonjean in Bertol.).

Frutice. Maggio. (Fiori bianchi).

Olivella it. - D. des alpes fr. 
D. cneorum Lin. - Presso Caselette e nei monti di San Michele della Chiusa.

Frutice. Giugno, Luglio. (Fiori rosei fragranti). Ceneoro it. - D. camélée fr.

\section{Ordine LXXXIV - Santalacee \\ (Santalaceae R. Brown). \\ GEN. Thesium Lin.}

* Foglie trinervie.

Th. intermedium Schrad. (Th. linophyllum RE-LiN. in parte). - Intorno alle selve: presso Susa alle Blaccie.

Per. Luglio, Agosto.

Alchimilla linaiola it. - Thésion intermédiaire fr.

Blaccie e Gravere (Negri). Cenisio (Bonjean in Bertol.)

" Foglie uninervie, o oscuramente trinervie.

Th. alpinum Lrs. - Pascoli del Cenisio.

Per. Giugno, Luglio.

Casia alpina it. - T. des alpes fr.

* Th. pratense EhrH. - Altipiano del Cenisio (Negri).

Ann. Giugno, Luglio.

Ordine LXXXV - Eleagnee (Eleagneae R. Brown.). GEN. Hippophae Lin."

H. rhamnoides Lin. - Frequentissimo nei siti sabbiosi lungo la Dora.

Frutice. Maggio.

Olivella spinosa it. - Argousier faux-nerprun fr. 


\section{Ordine LXXXVI - ARistolochiaceE}

(Aristolochiene Juss.)

A. rotunda Lin. - Aristolochicae rotundae radix Off. - Strologia rotunda vern. - Siti montani e specialmente nel monte di Caselette letto Musine. Stimolante emenagoga.

Per. Maggio. (Radice tuberosa).

Aristolochia rotonda it. - Aristoloche ronde fr.

A. clematitis Lin. - Aristolochia vulgaris Off. - Comunissima nelle vigue presso Susa. Emenagoga stomatica.

Per. Maggio, Giugno. (Kadice non tuberosa).

Aristolochia clematite it. - A. clematite, Sarasine fi.

A. rotunda e clematitis non sono piu in uso come rimedi. Esse fuor di dubbio sono acri, ed ingerite dalle bestie possono essere cagione di disturbi gastrici.

\section{Ordine LXXXVII - EmpetraceF}

(Empetraceae Lindl.).

\section{Gen. Eanpedrum LiN.}

E. nigrum Lrs. - Siti freddissimi del Cenisio. Fruticoloso. Giugno, Luglio. (Fiori rosei). Erica baccifera it. - Camarine à fruits noirs fl: 


\section{ORdiNe LXXXVIII - EUforbiaceE \\ (Euphorbiaceae Juss.)}

GEN. 1. - Euploabia Lin.

Sez. $0^{2}$ - Foglie opposte stipulate : ghiandole dell'involuero semilunari.

E. lathyris Liv. - Lathyris o Catapuccia Off. Volgare negli orti. Acre, emetica, drastica.

Ann. Giugno, Agosto.

Cutapuzia it. - Giallaria, erba purga o topa vern. Euphorbe épurge fr.

\section{Sez. $2^{2}$ - Foglie sparse.}

* Ghiandole dell'involucro arrotondite.

E. helioscopia Lrs. - Abbonda nelle vigne e nei siti incolti.

Ann. Giugno, Agosto.

Erba calenzola it. - Rogna daso vern. - E. reveillematin fr.

E. verrucosa LaMk. - Prati e pascoli macilenti. Molto lodata nelle febbri intermittenti, ma drastica da essere ordinata da un medico cauto.

Per. Maggio, Giugno.

Titamalo dolce it. - Laitin vern, - E. à verrues ir.

Nel testo E. verrucosa Lin.

E. platyphylla Lin. - Tithymalus platyphylla Arvensis 0ff. - Nei siti incolti e nei campi.

Ann. Luglio, Agosto.

Titimalo palustre it. - Erba dle scarvasse vern. - E. à larges feuilles fr.

Nel testo E. platyphyllos dal Murr. Syst. 
* E. pilosa Lrn. - A Caselette ai piedi del Musinè (Gras) (Balbis in Parlat.).

Per. Maggio, Luglio.

Titimalo peloso it. - E. poilu fr.

E. dulcis Lrs. - Tithymalus dulcis o Esula dulcis Off. - Qua e là nelle selve.

Per. Aprile, Maggio.

Titimalo bitorzoluto it. - E. pourpré fr.

E. gerardiana JACQ. (E. seguierii RE-ALL.). - Frequente nei siti sabbiosi e sassosi lungo la Dora. Per. Maggio, Agosto.

Esula linoide it. - E. de Gérard fr.

* Ghiandole dell'involucro semilunari.

E. peplus Lis. - Non rara nei siti ombrosi intorno alle case e agli orti.

Ann. Maggio, Agosto.

Calenzola piccola it. - E. des vignes fr.

* E. sulcata De Less. - Su la Brunetta (Cesati) (Negri).

Ann. Aprile, Maggio.

Titimalo a semi solcati it. - E. sillonné fr.

E. saxatilis JAcQ. - Trovata dal ch. Bellardi in siti sterili e sassosi presso Susa lungo la via della Novalesa.

Per. Maggio, Luglio.

Titimalo rupino it.

Nel testo E. saxatilis Lin. dal Murr. Syst. 
Pianta dubbia come pertinente alla nostra flora: leggasi quanto scrive il Colla sul proposito, Herb. Vol. V, pag. 124: ne il Bertoloni, il Parlatore, e il Compendio Fl. it. Cesati, etc.. accennano a località piemontesi.

\section{E. cyparissias Lis. - Ovunque nei pascoli e} lungo la Dora. Acre, caustico come tutte le specie di Euforbia.

\section{Per. Aprile, Luglio.}

Erba cipressina it. - Lait d' serpent, piumassera dle vache vern. - E. petit cyprès, rhubarbe du paysan fr.

E. falcata Lin. - Trovata da Ignazio Molineri nelle vigne presso Susa.

Ann. Giugno, Agosto.

Calenzola aristata it. - E. en faux fr.

E. exigua Lin. - Nelle vigne.

Ann, Maggio, Agosto.

Esola sottile it. - E. fluet, petite ésule fr.

E. taurinensis ALL. - Frequentissima nelle vigne intorno Susa, ed essendo rara nei dintorni di Torino, stupisco come dal chiaro Allioni non sia stata chiamata piuttosto Euphorbia Segusiensis.

Ann. Maggio, Luglio.

Calenzola di Piemonte it. - E. de Turin fr.

Per buona ventura degli ammalati l'uso interno dell' Euforbie, tanto indigene che esotiche, $\dot{e}$ stato bandito dalla terapia moderna. Il succo lattiginoso irritantissimo posseduto da questo genere di piante puó servire, senza pericolo, solamente come epispastico.

Nei monti di Givoletto, precedentemente nominati (pag. 225 e 227, vegeta l'Euphorbia insularis Boissier (Euphorbia hiberna Viv. Bertol, Grenier e Godron, non Lin. sec. Parlat. 
Fl. it., IV, pag. 462). Questa specie creduta affatto meridionale (Appennino genovese, Corsica e Sardegna) fu rinvenuta, non sono molti anni, a Givoletto dai giardinieri dell Orto Botanico Torinese, Chiuso e De Filippi.

La sua esistenza nei monti di Givoletto prova quanti problemi di Geografia botanica sono ancora da risolvere.

\section{Gen. 2. - Buxus LiN.}

B. Sempervirens Lix. - Buxus Off. - Martel vern. - Nelle selve presso Sinsa. Fogrlie e legno amaro e surloriferi.

Frutice. Aprile.

Bossolo, bosso it. - Buis toujours vert fr.

Farmaco disusato.

* (tin. 3) - Mereurialín LIN.

M. annua Lis. - Ruderati e siti colti.

Aun. Giugno, Agosto.

Mercorella it. Mercuriale annuelle fr.

97. perennis Lix. - - Insinè alla cima presso i rottimi del seguale trigonometrico.

Per. Maggio, Giugno.

Mercorella bastarda it. - M. vivace fr.

$1 l$ gen. Mercurialis non è riportato nella flora segusiensis, evidentemente per dimenticanza.

()RHAE LXXXIX - ORticacee (Urticeae Juss.). GEN. 1. - Urtica LIN.

U. urens Lis. - Urtica urens minor Off. - Urtie vern. - Intorno alle case; nei ruderati. Farmaco disusato.

Ann. Luglio, Settembre.

Ortica piccola it. - Ortie brûlante fr. 
U. dioica Lis. - Urtica urens maxima Off. Tolgare nelle siepi e negli orti. Astringente.

Per Maggio, Luglio.

Ortica it. - 0 . dioïque fr.

Le ortiche sono abbandonate come farmaco : servono oggi, cotte, ad alimentare i piccoli tacchini e i suini.

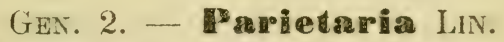

P. officinalis Lrx. - Parietaria, Helxine, Vitraria Oif. - Panataria vern. - Su le mura. Emolliente.

Per. Luglio, Settembre.

- Erba vitriola it. - Pariétaire officinale fr.

La Parietaria contione gran quantità di nitrato di potassa, e da taluni medici anche presentemente viene ordinata per combattere la idropisia.

\section{GEN. 3. - Humalas Lin.}

H. Pupulus Lix. - Lupulus Off. - Luvertin vern. - Nelle siepi. Gli amenti sono amari, stomachici.

Per. Luglio, Agosto.

Luppolo it. - Houblon grimpant fr.

Il luppolo è coltivato estesamente in Germania, in Inghilterra e in Francia, e il suo frutto viene adoperato a dare l'amaro e l'aroma alla birra.

\section{* Gen. 4. - Canmabis Lin.}

C. sativa Lis. - Coltivata : è originaria dell'India. Ann. Luglio, Agosto.

Canapa it. - Cauna vern. - Chanvre cultivé fr. 
302

\section{Gen. 5. - Ficus LIN.}

F. carica Lin. - Caricae fructus Off. - Fighè vern. - Nelle vigne e nelle rupi. Nutriente emol. liente espettorante.

Arbusto. Luglio, Agosto.

Fico it. - Figuier commun fr.

Il Fico sta fra gli alimenti cosi detti zuccherini e respiratori: è usato anche esternamente per fomento suppurativo.

\section{* Gen. 6. - Morus Lin.}

M. alba Lin. - Coltivato per alimento del baco da seta. Originario della China.

Albero. Maggio.

Gelso bianco it. - Morè vern. - Mûrier blanc fr.

\section{Gen. 7. - Celtis Lin.}

C. australis Lis. - Falagrèe vern. - Non rars presso Susa e in tutta la provincia nei siti incolti. Arbusto. Maggio.

Fragiraco it. - Micocoulier du midi fr.

A Caselette.

Gen. 8. - Ulmag LiN.

U. campestris LiN. - Ulmus Off. - Ourm vern. - Vegeta spontaneo a Condove nel sito detto la Grangietta. Corroborante, astringente.

Albero. Aprile, Maggio.

Olmo it. - Orme champêtre fr.

Rimedio disusato. La corteccia è tannica e fu quindi adoperata un giorno come astringente. L'olmo coltivato è un albero bello ed utile ad un tempo per lavoro. 
Ordine XC - Juglandee (JugZandeae DC.).

\section{* Gen. Juglams LIN.}

J. regia Lin. - Coltivato. Del Caucaso meridionale; notissimo tanto pel suo frutto, che pel legname eccellente per lavoro.

Fiorisce in Maggio.

Noce it. - Nousera vern. - Noyer commun fr.

Le foglie fresche del noce in decozione sono raccomandate nei disturbi digestivi della scrofolosi. Dal frutto si estrae un olio conosciuto in pittura e anche usato per condiments invece dell'olio d'olivo.

Ordine XCI. - Cupolifere (Cupuliferae Rich.).

Gen. 1. - Fagas Lin.

F. sylvatica Lris. - Fagus 0ff. - Fò vern. Comune nelle selve. Dai semi si può ricavare un olio, il quale serve agli usi domestici.

Albero. Maggio.

Faggio it. - Hêtre commun fr.

\section{Gen. 2. - Castanea Tournf.}

c. vulgaris LAMk. (Fagus castanea RE-Lin.) - Castaneae fructus Off. - Castagnè vern. - Ovunque.

Albero notissimo. Giugno.

Castagno it. - Châtaignier commun fr. 


\section{Gen. 3. - Quereas LiN.}

Q. robur Lin. Fl. suec. - Quercus o Robur Off. - hôl vern. - Abbonda nelle selve. Corroborante stomatico.

Albero. Aprile, Maggio.

Farnia it. - Chêne-liége fr.

(Q. pedunculata Ehrh.).

Q. cerris Lix. - Anche nelle selve.

Albero. Maggio.

Cerro it. - Sarron vern. - Ch. cerris fr.

La corteccia di Quercia abbonda di acido tannico, e nella sua azione rassomiglia al tannino: ha pero un effetto più debole, ed inoltre presa internamente disturba oltremodo la digestione. Quindi il suo uso oggi è limitato ad applicazioni esterne in tutti quei casi in cui conviene il tannino. (Nothnagel).

\section{Gex. 4. - Coryllus Lin.}

C. avellana LiN. - Avellana Off. - Ninsole vern. - In tutte le selve.

Arbusto. Febbraio, Marzo.

Avellana it. - Coudrier aveline fr.

\section{Gen. 5. - Carpinans Lin.}

C. betulus Lrn. - Cherpo vern. - Nei siti ombrosi.

Arbusto. Aprile, Maggio.

Carpino it. - Charme commun fr. 
Ordine XCII - SALICINeE (Salicineae Rich.)

\section{GEN. 1. - alix Lin.}

Amenti laterali, cioè sviluppantisi da gemme lungo i rami.

1) Squame degli amenti piú o meno sbiadite non nereggianti all'apice.

S. pentandra LIN. - Cenisio.

Frutice. Maggio, Giugno. (Squame caduche).

Salcio odoroso it. - Saule à cinq étamines fr.

Cenisio presso il lago (Parlat.). Alpi di Exilles (Allioni).

S. alba Lin. - Salix vulgaris alba Off. - Lungo la Dora. Corteccia corroborante astringente e lodata come succedanea della china.

$\beta$ vitellina Parlat. (Salix vitellina Re-Lin.). - Nei siti umidi, e coltivata per attaccare le viti.

Albero. Aprile, Maggio. (Squame caduche).

Salcio salcio it. - S. blane fr.

S. fragilis Lin. - Nelle selve. La corteccia dei rami è amaro-stittica, corroborante astringente.

Arbusto. Aprile, Maggio. (Squame caduche).

Salcio fragile it. $-\mathrm{S}$, fragile fr.

S. amygdalina LrN. - Frequente lungo la Dora. $\beta$ concolor Parlat. (Salix triandra Re-Lin.). Lungo la Dora.

Arbusto. Aprile, Maggio. (Squame persistenti).

Salcio da far ceste it. - S. amandier fr.

$\grave{\mathrm{E}}$ appunto con questa specie che si fanno ceste. La varietà ha le foglie verdi nelle due pagine; la specie le ha glauche nella pagina inferiore. 
S. incana Schrank. (Salix viminalis Re-ViLL.). - Lungo la Dora.

Frutice. Marzo, Aprile. (Squame persistenti).

Vetrice bianca it. - $\mathrm{S}$. drapé fr.

E adoperata per far panieri, per vestire fiaschi, ecc. Non è buona a legare perchè fragile.

2) Squame degli amenti pit o meno colorate, e più di tutio all'apice ove spesso sono nereggianti.

S. purpurea Lin. (Salix Helix Re-Lin.). - Qua e ls presso la Dora.

Frutice. Marzo, Aprile.

Salicella it. - S. pourpré fr.

È adoperata a far panieri, culle, gabbie, etc.

* S. pontederana Willd. Parlat. - Cenisio. (Huguen. in Parlat.).

Frutice. Maggio.

Salcio tirolese it. - S. de Pontédéra fr.

S. hastata Lrv. ( Salix pontederae RE-VILL.). - Siti selvatici del Cenisio presso il lago.

Frutice. Giugno, Luglio.

Salcio silvano it. $-\mathrm{S}$. en fer de lance fr.

S. daphnoides Vill. - Lungo la ria nei prati del Cenisio.

Arbusto. Aprile, Maggio.

Salcio nero - S. Daphnê fr.

* S. lapponum LrN. - Cenisio (Parlat.). (Negri).

Frutice. Luglio.

Salcio salviastro it. - S. des Lapons fr. 
* S. glauca Lrs. - Cenisio (Balbis, Bonjean, Seringe in Bertol.). (Huguen. in Parlat.).

Frutice. Giugno, Luglio.

Salcio molle it. - S. glauque fr.

(Salix lapponum All, non Lin.).

S. myrsinites LIN. - Abbonda intorno al lago del Cenisio.

Frutice. Giugno, Luglio.

Mirsinito it. - S. myrte fr.

S. caesia VILl. (Salix myrtilloides Re-Wirtd. Alt. Auct.). - Intorno al lago del Cenisio.

Frutice, Giugno, Luglio.

Salcio cangiante it. - S. bleuâtre fr.

Nel testo S. myrtilloides Lin.

S. caprea Lin. - Ai Cenau presso Susa. Corteccia corroborante astring'ente.

Arbusto. Aprile, Maggio.

Salica it. - S. Marceau fr.

* S. arbuscula Lin. - Piano del Cenisio (Negri). Cenisio presso la vecchia strada che conduce dal $5^{\circ}$ ricovero alla Gr. Croce: e in abbondanza vicino al lago e al Reservoir (Parlat.).

Frutice. Giugno, Luglio.

S. arbuste fr.

* S. glabra Scop. - Cenisio presso il lago (Parlat.).

Frutice. Maggio, Giugno.

Salcio silvano it. - S. noircissant fr. 
Amenti terminali, cioł sviluppantisi all'apice dei rami.

Frutici nani distesi per terra.

\section{S. reticulata Lin. - Cenisio.}

Giugno, Agosto. (Foglie a peziolo lunghetto).

Salcio reticolato it. - S. reticulé fr.

Assietta, alpi di Bussolino alla Balmetta superiore.

S. retusa Lin. - In tutte le alpi.

Luglio, Agosto. (Foglie brevemente peziolate).

Salcio sermellino it. - S. émoussé fr.

Altipiano del Cenisio (Negri). A Ronche (Parlat.).

S. herbacea LrN. - Pascoli umidetti freddi del Cenisio.

Giugno, Luglio. (Foglie brevemente peziolate).

Salcio sassatile it. - S. en herbe fr.

Altipiano del Cenisio (Negri). A Ronche (Parlat.)

Questa specic rappresenta la vegetazione legnosa nelle altissime regioni alpine presso i ghiacciai e le nevi perpetue.

Principii attivi della corteccia di vari salici sono l'acido tannico e la salicina. Quest' ultima, scoperta nel 1825, fu creduta capace di sostituire i sali di china : ma ció non è, ed oggi è ritenuto che non si possono stabilire confronti trn la salicina e la chinina.

\section{Gen. 2. - Populus LiN.}

- Squame degli amenti cigliate.

P. alba LrN. e 0ff. - Arbron vern. - Presso le acque. Farmaco disusato.

Albero. Marzo, Aprile.

Pioppo bianco it. - Peuplier blanc fr.

P. tremula Lis. - Termo vern. - Intorno alle selve.

Albero. Aprile, Maggio.

Albarella it. - P. tremble fr. 
" Squame glabre.

P. nigra Lin. e Off. - Volgarissimo nelle selve lungo la Dora. Farmaco disusato.

Albero. Marzo, Aprile.

Pioppo nero it. - P. noir fr.

* P pyramidalis Parlat. Populus piramidalis Bertol.). Pioppo cipressino it. - P. pyramidal fr.

Con la specie.

Le gemme del P. alba e del nigra furono adoperate un giorno come rimedi contro la dissenteria e l'artritide, oggi sono disusate. Servirono pure a preparare il cosi detto Unguento di pioppo o populeo delle farmacie.

Le foglie dei pioppi bianco e nero sono mangiate con piacere dal bestiame, e danno un legname facile al lavoro ma non molto resistente alla umidità.

\section{Ordine XCIII - Betulinee (Belulinetle Rich.)}

\section{Gen. 1. - Befrala Lin.}

B. alba Lin. - Betula Off. - Biola vern. Ovunque nei siti montani ed alnestri: frequente al Cenisio. Il succo è rimedio dei mali di pelle.

Albero. Aprile, Maggio.

Betula, Bedollo it. - Bouleau blanc fr.

L'olio di Betula, che si ottiene dalla corteccia di quest'albero, è rimedio pregevole in talune malattie cutanee. (Can$\operatorname{tani).}$

* B. pubescens Eнrн. - Cenisio nella foresta di larici e di abeti che sovrasta a Lanslebourg ai confini della Flora italiana (Parlat.).

Alberetto. Aprile, Maggio.

Betula pelosa it. - B. pubescent fr. 


\section{Gen. 2. - Imams Tournf.}

A. glutinosa Gaertio. (Betula alnus a glutinosa RELin.) - Alnus 0ff. - Verna vern. - Abbonda nei siti umidi presso i torrenti e i fiumi. Farmaco disusato.

Albero. Marzo, Aprile.

Ontano it. - Aulne glutineux fr.

Il legname dell'Ontano resiste all'azione della umidità ; sotto terra in siti palustri permanet immortalis ad aeternitatem, scrisse Vitruvio.

Le foglie son mangiate dal bestiame.

A. viridis DC. (Betula viridis Re-BeLL.). - Nel Cenisio e sopra il Bosco nero.

Frutice. Aprile, Maggio.

Ontano verde it. - A. vert fr.

Assietta: Sacra di S. Michele.

\section{Ordine XCIV - Conifere (Coniferne Juss.) \\ * Gen. 1. - Epliedra Lin.}

* E. distachya Lin. - Su la Brunetta (Rostan in Parlat.).

Frutice. Maggio, Giugno.

Uva marina it. - Ephèdre à deux épis fr.

\section{Gen. 2. - Taxus Lin.}

T. baccata Lrs. e Off. - Nelle selve sopra la Novalesa. Velenosa.

Arbusto. Marzo, Aprile.

Tasso it. - If commun fr.

Le foglie sono mortali specialmente pei Solipedi. 


\section{GEN. 3. - Imiperus Lin.}

* Foglie con una glandola sul dorso.

J. sabina LiN. - Sabina Off. e vern. - Sopra Le Ferrieres. Stimolante, emenag’og’a, antielmintica.

Alberetto. Maggio, Giugno.

Sabina it. - Genévrier des alpes, Sabine fr.

La Sabina è velenosa, e non è questo il luogo di enumerare uno per uno i casi nei quali la terapia cauta e prudente può farne uso. È l'abortivo più sicuro ma il più pericoloso per le conseguenze che può produrre. Per lo passato in alcuni Stati d'Italia erane vietato lo spaccio anche ai farmacisti.

* Foglie senza glandola sul dorso.

J. communis Lin. - Juniperus Off. - Geneiver vern. - In tutti i pascoli sterili montani e intorno alle selve. Le bacche sono stimolanti, diuretiche, stomachiche.

Frutice. Aprile.

Ginepro it. - G. commun fr.

* $\beta$ nana (J. nana Willd.) - All'Assietta.

Le bacche di ginepro sono prescritte presentemente come digestive e carminative, sovra di tutto come diuretiche (Cantani).

J. oxycedrus LIN. - Nelle rupi tra Monpantero e Foresto.

Frutice. Marzo, Aprile.

Ginepro rosso it. - G. oxycèdre fr.

Tra Susa e Bussolino All. Auct., pag. 35. 


\section{GEN. 4. - Eimus Lin.}

* Foglie gemine.

P. sylvestris Lis. e Off. -- Volgare nei monti. Le gemme sono stimolanti diuretiche.

Albero. Maggio.

Pino comune it. - Pin sylvestre fr.

(Pinus Mughus Jacq.).

* P. montana Duror. - Cenisio, foresta sopra Lanslebourg vicino alla Ramasse (Parlat.).

Albero. Giugno, Luglio.

Pino di montagna it. - P. de montagne fr.

(Pinus mughus Scop. P. uncinata Ram.).

* Foglie riunite per cinque, rar. meno o pit, mai gremine.

P. cembra Lis. - Frequente nel Cenisio e nei monti di Oulx. Resina liquida stimolante.

Albero. Luglio, Agosto.

Pino zimbro it. - P. cembra, arole fr.

* Foglie fascicolate per molte.

P. Iarix Lin. - Larix 0ff. - Meleso veru. Ovunque nelle selve alpestri. Virtù del precedente. Albero. Maggio.

Larice it. - P. mélèze fr.

Albero assai noto, grandioso, bello ed utile per molti riflessi, ma che disgraziatamente la scure cieca e vandalica abbatte, e giorno per giorno rende più rado su le nostre alpi.

Dal larice inciso in basso del tronco sgorga la resina che vien chiamata in commercio e nelle farmacie terebentina di Venezia. 
Foglie solitarie.

P. abies Lrs. - Abies foemina Off. - Nel Cenisio e nei monti di Oulx. Le gemme sono stimolanti diuretiche, e la sua resina è conosciuta nelle officine sotto il nome di pece di Borgogna.

Albero. Aprile, Maggio.

Abete rosso it. - Sapin fr.

(Abies excelsa DC.).

P. picea Lin. - Abies mas Off. - Nelle selfr sopra la Novalesa. Resina stimolante.

Albero. Maggio, Giugno.

Abete maschio it. - Sapin blane fr.

(Abies pectinata DC.).

Liazione delle gemme dei pini è diurotica come di queile del Ginepro. Le resine sono stimolanti, ed è svariato il loro uso farmaceutico interno ed esterno, allo stato naturale. decomposte, o combinate con altre sostanze. 



\section{ClaSse II. - MONOCOTILEDONI}

(Monocotyledoneae)

Ordine XCV - Alismacee (Alismaceae Juss.)

Gen. Alisma Lin.

A. plantago Lin. - Volgare nelle acque stagnanti e alle sponde dei laghi.

Per. Giugno, Luglio.

Alisma it. - Alisma plantain fr.

Negli stagni da S. Ambrogio alla Chiusa, e di Caselette.

A. ranunculoides Lrs. - Vegeta nei siti medesimi. Per. Maggio, Giugno.

Petacciola ranunculina it. - A. renoncule fr.

Ordine XCVI - Butosiacee (Butomeae Rich.)

\section{Gen. Butomus Lin.}

B. umbellatus Lin. - Intorno ai laghi di Avigliana su la fede del ch. Allioni.

Per. Maggio.

Giunco fiorito it. -- Butôme en ombelle fr. 


\section{Ordine XCVII - Giuncaginacee}

(Juncagineae Rich.)

\section{Gen. 1. - Triglochim LIN.}

T. palustre Lin. - Siti palustri presso i laghi di Avigliana, presso la Dora e a Susa.

Per. Aprile, Maggio.

Giuncastrello it. - Troscart des marais fr.

* Gen. 2. - Sellenclizenia Lin.

S. palustris Lıx. - Siti paludosi del Cenisio (Huguen. in Parlat.).

Per. Maggio, Giugno.

Scheuchzérie des marais fr.

Ordine XCVIII - Potanacer (Potameae Juss.)

GEN. 1. - Potramogedom LIN.

- Foglie superiori galleggianti oppnste.

P. natans Lin. - Acque a lento corso ai laghi di Avigliana.

Per. Maggio, Luglio.

Lingua d'acqua it. - Potamot nageant fr.

"Foglie tutte sommerse di varia forma, dalla lanceolata alla sub-rotondia, salvo la lineare, alterne, le fiorali opposte.

P. lucens Liv. - Laghi di Avigliana.

Per. Luglio, Agosto.

Brasca it. - P. luisant fr. 
P. crispus Lis. -- Laghi di Avigliana.

Per. Maggio, Giugno.

Lattuca vanina it. - P. crispé fr.

Foglie tutte sommerse lineari, o tutte alterne o le fiorali opposte.

P. pusillus Lrs. - Lago del Cenisio.

Per. Luglio, Agosto. (Foglie fiorali opposte).

Gramigna acquatica sottile it. - P. fluet fr.

P. marinus Lin. - Trovato dal chiaro Allioni nel lago del Cenisio.

Per. Luglio, Agosto.

Fieno di palude it. - P. marin fr.

(P. filiformis Pers.).

... Foglie tutte sommerse, e tutte opposte lanceolate

o lanceolate lineari.

P. densus Lin. - Siti acquosi presso le fonti.

$\beta$ serratus ( $P$. serratus RE-LIN.). - Trovato dal chiaro Allioni nel lago del Cenisio.

Per. Maggio, Luglio.

Erba gala it. - P. à feuilles opposées fr.

Il Colla scrive che il P. serratus non fu più trovato, dopo Allioni, nel lago del Cenisio.

\section{Gen. 2. - Zamaielellia LIN.}

Z. palusiris Lin. - Nelle acque a lento corso.

Per. Maggio, Giugno.

Alga di chiana it. - Zannichelle des marais fr. 


\section{Ordine XCIX - Lemnacee (Lemnacede Dub.)}

\section{Gen. 1. - Lemna LIN.}

(Radici cellulari)

L. trisulca Lrs. - Nelle fonti e intorno ai laghi di Avigliana.

Ann. Aprile, Maggio. (Frondi lanceolate).

Erba pulla it. -- Lenticule prolifère fr.

L. minor LiN. - Frequente nelle fonti a lento corso.

Ann. Aprile, Maggio. (Frondi lenticolari).

Lente palustre it. - L. mineure fr.

\section{Gen. 2. - Ipirodella Schleid.}

(Radici percorse da un vaso spirale)

Sp. polyrrhiza Schleid. (Lemna polyrrhiza RE-LiN.). - Acque stagnanti.

Ann. Aprile, Maggio. (Frondi sub-orbicolari).

Lente palustre it. - Lenticule à plusieurs racines ìr.

\section{Ordine C - Tifacee (Typhaceae Juss.)}

\section{Gen. 1. - Typlea Lin.}

T. Iatifolia Lin. - Laghi di Avigliana.

Per. Maggio, Giugno.

Mazza sorda it. - Massette à larges feuilles fr.

T. angustifolia LIN. - Presso la Dora in siti sabbiosi umidetti.

Per. Giugno, Luglio.

Sala it. - M. à feuilles étroites fr. 
GEN. 2. -- Gpand

S. ramosum Hudson. ( Sp. erectum RE $\propto$ LiN.). Siti acquosi.

Per. Maggio, Giugno.

Sparganio it. - Rubanier rameux fr.

S. natans Lin. - Cenisio nel piccolo lago sopra la piana di S. Niccolà.

Per. Luglio, Agosto.

Sparganio minuto it. - $R$. flottant $f r$.

Cenisio al Baraccone (Parlat.).

Ordine CI - Aroidee (Aroideae Juss.)

GEN. A Ream Lin.

A. maculatum LiN. - Arum. Off. - Vidi questa specie lungo la via nei luoghi ombrosi e umidetti tra Almese e la Rivera. La radice fresca è acre, stimolante, epispastica.

Per. Aprile, Maggio.

Gichero it. - Gouet commun fi.

Le radici tuberose del Gichero hanno le qualità notate dall'autore, ma non sono più usate in farmacia.

A. dracunculus LIN. - Dracunculus, Dracontium - Serpentaria Off. - Alle Blaccie presso Susa.

Per. Giugno.

Dragonzio it. - G. serpentaire fr. 


\section{ORDiNe CII - ORCHIDEE (Orchideae Juss.)}

40.03. (cadicul6)

A) Labello speronato.

1) Ogni caudiculo provvisto di glandola (retinacolo) propria.

\section{GEN. 1. - Decthis LiN.}

Divisioni esterne iel perigenio conniventi e tignranti an elmo: tuberi interi.

a) Labello intero.

Q. papilionacea LiN. - $\beta$ rubra Parlatr. (0. rubra REAur. Auct.). - Nei pascoli sterili di Caselette e di Almese.

Per. Aprile, Maggio.

Cipressini it. - Orchis papilionacé fr.

Selve di Alpignano e del Musinè (Gras;.

Nel testo Orchis rubra Lin.

b) Labello trilobato o trifdo.

0. morio LrN. - Abbonda nei pascoli sterili intorno Susa.

Per. Aprile, Maggio.

- Gigli caprini it. - - 0. bouffon fr.

0. coriophora Lin. - Nei pascoli presso Caselette e sopra Monpantero.

Per. Aprile, Maggio.

Cipolla cimiciattola it. - 0 . punaise fr.

0. ustulata LrN. - Qua e là nei prati poco erbosi.

Per. Maggio, Giugno.

Giglio macchiettato it. - 0 . punaise fr. 
0. tridentata Scop. (0. variegata RE-AlL.). - Pascoli erbosi.

Per. Maggio, Giugno.

Giglio screziato it. - 0 . tridenté fr.

Sagra di S. Michele.

0. militaris LrN. Fl. suec. - Pascoli poco erbosi presso Susa: nei prati di Monpantero andando alla chiesa detta Madonna della Coà.

Per. Maggio, Giugno.

Giglio crestato it. - 0. militaire fr.

** Divisioni esterne del perigonio patenti.

1) Tuberi intieri.

* O. laxiflora Lamk. - Frequente nei prati presso Caselette (Re, Fl. Tor.).

Per. Giugno, Luglio.

Giglio di prato it. - o. à fleurs lâches fr.

0. pallens Lrn. - Cenisio: presso Susa, e nei monti di Giaveno.

Per. Maggio.

Giglio puzzolente it. - 0. pâle fr.

0. mascula LIN. - Prati alpini e montani : frequente alla Comba sopra Chianoc e nei prati alpini di Monpantero.

Per. Aprile, Maggio.

Giglio caprino it. - 0. mâle fr. 
2) Tuberi palmati.

0. sambucina Lrv. - Nei pascoli e nei prati pingui montani.

Per. Maggio, Giugno.

Giglio sambucino it. - 0 . ̀̀ odeur de Sureau fr.

Piano del Cenisio (Bouvier) Sagra di San Michele, Musiné (Gras).

0. latifolia Lin. -- Intorno al lago del Cenisio: nei prati di Codizar sopra Chiomonte, e presso Susa nei prati umidi di Monpantero superiore.

Per. Giugno, Luglio.

Orchide palmata, sconcordia it. - O. à larges feuilles fr'. Sagra di S. Michele nei prati (Gras).

0. maculata Lis. - Nei prati umidi montani.

Per. Giugno, Luglio.

Concordia, Palmachristi it. - o. tacheté fr.

0. incarnata LiN. Fl. suec. - Trovai in abbondanza questa specie nei prati montani di San Giorgio sotto la borgata detta dei Bonnet. Si rinviene parimenti nel Cenisio, nei monti di Giaveno e altrove.

Per. Giugno, Luglio.

Orchide carnicina it. - 0 . incarnat fr.

\section{Gen. 2. - Gymmademia R. Brown.}

G. odoratissima Ricr. (Orchis odoratissima Re-Lix.). - Intorno ai laghi di Avigliana e nei prati tra Foresto e San Giuliano.

Per. Giugno, Luglio.

Orchide odorosa it. - Gymnadénie très-odorante fr. 
G. conopsea R. Brown. (Orchis conopsea Re-Lin.). Abbonda nei prati del Collet andando a Fenestrelle. Per. Giugno, Luglio.

Orchide garofanata it. - G. moucheron fr.

\section{GeN. 3. - Pieclloia Parlat.}

R. albida Parlat. (Satyrium albidum Re-Lin.). Aipi di Giaveno.

Per. Luglio.

Testicolo fascicolato it. - Ricchia blanchâtre fr.

Altipiano del Cenisio (Negri sotto Gymnadenia albida Rich).

Gen. 4. - Nighoitella L. C. Rich.

N. angustifolia Rich. (Satyrium nigrum RE-LIN.): Abbonda nei prati alpini.

Per. Luglio, Agosto.

Palmachristi fragrantissima it. - Nigritelle à feuilles étroites fr.

N. globosa Reichs. Fl. exsc. (Orchis globosa Re-Lin.). - Fréquente nei prati alpini.

Per. Giugno, Luglio.

Orchide appendicolata it. - N. globuleuse fr.

Sagra di San Michele.

\section{Gen. 5. - Coeloglossum Hartu.}

G. viride Hartm. (Satyrium viride Re-Lin.). - Nei pascoli montani ed alpini.

Per. Giugno. (Sperone breve scrotiforme).

Testicolo di volpe it. - Orchis vert fr. 
GEN. 6. - Platanulaèra C. L. Rich.

P. bifolia Rich. (Orchis bifolia Re-LrN.). - Volgare nelle selve.

Per. Giugno, Luglio.

Cipolla di due foglie it. - Orchis à deux feuilles fr.

2) Una sola glandoli (retinacolo) comune ai due caudicoli.

\section{Gen. \%. - Amacamptis L. C. Rich.}

A. pyramidalis Rich. (Orchis pyramidalis RE-LiN.). - Nei pascoli presso Caselette : a Susa, alle Blaccie e nelle vigne di San Costanzo.

Per. Maggio, Giugno.

Orchide piramidale it. - Orchis pyramidal fr.

B) Labello non speronato.

1) Ogni caudicolo provvisto di glandola (retinacolo) propria.

\section{Gen. 8. - Ophrys Lin.}

a) Labello trifido o trilobo.

0. apifera Hudson. (Ophrys insectifera Re Intorno Susa.

Per. Maggio, Giugno.

Vesparia it. - Ophrys abeille fr.

Nei monti intorno ad Avigliana (Gras).

0. muscifera Hudson. (Ophrys myodes Re - Ophrys insectifera $\alpha$ Lin. - Intorno Susa.

Per. Maggio.

Pecchie it. - 0. mouche fr. 
b) Labello intero sub-quadro con l'apice smarginato-bilobo.

0. arachnites Host. (Ophrys arachnites Re - Ophrys insectifera $\eta$ LiN. - Intcrno Susa.

Per. Maggio.

Vesparia crestata it. - 0 . fausse araignée fr.

GEN. 9. - Tlenemintum R. Brown.

H. monorchis R. Brown. (Ophrys monorchis Re-LIN.). - Nei pascoli erbosi montani. Abbonda a Susa nei siti umidi tra la Brunetta e Monpantero.

Per. Maggio, Giugno. (Labello gozzato alla base).

Monorchide it. - Herminion caché fr.

GEN. 10. - Chamaedrehis C. L. Rich.

c. alpina Rich. (Ophrys alpina RE-LIN.). - Abbonda sul Cenisio.

Per. Luglio, Agosto.

Vesparia d'alpe it. - Chaméorchis des alpes fr.

Il Parlatore scrive invece (Fl. it., vol. 3, pag. 437) che questa specie è molto rara.

Cima di Ronche (Negri).

2) Una sola glandola (retinacolo) comune ai duo caudicoli.

GEN. 11. - Senpapias LIN.

S. longipetala Pollin. (Serapias lingua $\operatorname{RE} \propto$ LIN. Parlat.). - Nei prati macilenti.

Per. Maggio, Giugno.

Satirio incappucciato it. - Serapias a longs petales fr. 
Sez. $2^{2}$ - Mrasse polliniche sprovviste di pedicillo (caudicolo).

A) Labello lungamente speronato articolato.

\section{Gen. 12. - Limodorume Swartz.}

L. abortivum (Orchis abortiva RE-LIN.). - Intorno Susa.

Per. Maggio, Giugno.

Limodoro it. - Limodore à feuilles avortées $\mathrm{fl}_{\mathrm{l}}$.

B) Labello non speronato articolato.

Gev. 15. - Ceplealanthera L. C. RICH.

* C. ensifolia Rıch. -- Musiné nel sito detto Bric di Rivoli. (Re, Fl. Tor.).

Per. Maggio.

Elleborina bianca it. - Céphalanthère à feuilles en glaive $\mathrm{fr}$.

G. paliens Rrch. (Serapias lancifolia RE-Murr. Syst. - Siti montani presso le selve.

Per. Maggio, Giugno.

Elleborina giallognola it. - C. blanchâtre fr.

Nel testo S. lancifolia Lin.

C. rubra Rich. (Serapias rubra Re-Lin. Syst.). Abbonda nei castagneti di Meana presso la borgata detta delle Gran Traverse, e in luoghi simili è frequente nella contrada di Fossimagna.

Per. Maggio, Giugno.

Elleborina rosea it. - C. rose fr. 
Gen. 14. - Epipaetis L. C. Rich.

E. latifolia ALt. (Serapias latifolia RE-Lin. Mant.). - Pascoli montani intorno alle selve.

Per. Giugno, Luglio.

Elleborina crestata it. - Epipactis à larges feuilles fr.

E. palustris Rautz. (Serapias longifolia Re-Lin. Mant.)

- Frequente nei pascoli e nei prati umidi.

Per. Giugno, Luglio.

Mughetti pendolini it. - E. des marais fr.

C). Labello nè speronato nè articolato.

Gen. 15. - Listera Rob. Brown.

L. ovata Roв. Brown. (Ophrys ovata Re-Lin.). Ophrys o Bifolium Off. - Volgare nelle selve. La radice è nutriente.

Per. Maggio, Giugno.

Orchide di primavera it. - Listera ovale fr.

Nei tuberi di parecchie specie di Orchis esotiche ed indigene trovansi amido e sali, ma sopratutto una sostanza mucilaginosa, Salep delle farmacie.

È da marcare che la Listera ovata è l'Orchidea meno ricca di amido e di salep.

Gen. 16. - Neettia L. C. Rich.

N. nidus avis Rich. (Ophrys nidus avis Re-Lin.). Rinvenni questa specie nelle selve montane ombrose di Bussolino.

Per. Maggio, Giugno.

Nido d'uccello it. - Néottie nid-d'oiseau fr.

Detta nidus avis dalla forma delle fibre del rizoma che danno la figura di un nido d'uccello. 


\section{GeN. 1\%. - Spiramthes L. C. Rich.}

S. aestivalis Rich. (Ophrys aestiva Re-Balbis). Prati umidi di Caselette e intorno ai laghi di Avigliana.

Per. Luglio.

Viticcini di estate it. - Spiranthe d'été fr.

S. autumnalis Rich. (Ophrys autumnalis RE-BALBIS). - Pascoli aridi di Condove alla Roccea: presso Caselette e nei castagneti tra Villar di Basse e Avigliana.

Per. Luglio, Agosto.

Teśticolo odoroso it. - S. d'automne fr.

Testicolo fascicolato, di volpe, odoroso! Giudichi il lettore se questi siano nomi decenti e poetici, quali doviebberc essere quelli dei fiori spontanei.

\section{Gen. 18. - Goodyera R. Brown.}

G. repens Rob. Brown. (Satyrium repens Re-Lin.). - Trovata da Ignazio Molineri presso Oulx nel luogo detto le Sappé.

Per. Luglio, Agosto.

Elleborina serpeggiante it. - Godyère rampante fr.

\section{Gen. 19. - Corallorriliog Haller.}

C. innata Rob. Brown. - Cenisio (Bouvier). Cenisio verso Lanslebourg (Parlat.).

Per. Luglio, Agosto.

Radice corallina it. - Coralline parassite fr. 


\section{ORdine CIII - IRIDEE (Irideae Juss.)}

\section{Gen. 1. - Crocus Lin.}

C. vernus Alt. - In tutti i prati montani ed alpini.

Per. Maggio, Giugno.

Zafferano selvatico it. - Safran printanier $\mathrm{fr}$.

\section{Gen. 2. - Gladiolus LIN.}

G. segetum GawL. (Gladiolus communis RE-LiN. (?) ALr.). - Glayeul vern. - Nelle messi, e specialmente nei campi di Bussolino nella contrada detta S. Pietro.

Per. Maggio.

Gladiolo it. - Glayeul des moissons fr.

Gen. 3. - Irig Parlat.

i. germanica Lis. - Ireos nostratis radix Off. -Nelle rupi di S. Martino ad oriente di Susa. Acre, purgativa, diuretica.

Per. Maggio.

Giglio pavonazzo it. - Iris germanique, flambe fr.

Del rizoma dell'Iris germanica si tiene oggi poco o nessun conto in farmacia e in profumeria. Esso è cattivo succedaneo del rizoma dell'Iris florentina nota pel suo forte odore di violetta.

* I. pallida Lamk. - Musiné (Re, Fl. Tor.).

Per. Maggio, Giugno.

Ghiaggiolo odoroso it. 
330

\section{Gen. 4. - Xiplioion Parlat.}

X. pseudu-acarus ParL. (Iris pseudo-acarus Re-Lin.). - Acori palustris radix 0ff. - Presso i laghi di Avigliana.

Per. Maggio, Giugno.

Acoro falso.it. - Iris faux-acore fr.

\section{Ordine CIV. - AmarillideE (Amaryllideae Roz. Broron.) \\ Gen. 1. - Nareissus Lin.} (Perigonio coronato).

N. poeticus Lin. - Pantecoste vern. - Frequentissimo nei prati.

Per. Maggio.

Narcisso it. - Narcisse des poëtes fr.

N. pseudo-narcissus Lin. - Nelle selve apriche delle Blaccie e presso Susa nella contrada San Francesco.

Per. Maggio.

Narcisso trombato it. - N. faux-narcisse fr.

\section{Gen. 2. - Leucointm LIN.}

(Perigonio senza corona).

\section{L. vernum Lin. - Siti umidetti.}

Per. Marzo, Aprile.

Narcisso a campanelle it. - Nivéole printanière fr. 
Ordine CV. - Asparagacee (Asparageae Juss.)

A). Stili saldati tra loro.

* Gen. 1. - Asparagus Lin.

* A. officinalis Lrs. - Coltivato.

Per. Maggio, Giugno.

Asparago it. - Spars vern. - Asperge officinale fr.

Gen. 2. - Streptopens L. C. Rich.

S. amplexifolius DC. (Uuularia amplexifolia RE-LiN.). - Selve ombrose al lago del Cenisio.

Per. Luglio, Agosto.

Lauro alessandrino it. - Streptope embrassant fr.

Gen. 3. - Polygomatum Tournf.

P. verticillatum Alt. (Convallaria verticillata Re-Lin.) - Cenisio.

Per. Maggio, Giugno.

Ginocchietto stellato it. - Polygonatum verticillé fr.

P. officinale ALL. (Convallaria polygonatum RE-LIN.). - Polygonatum o Sigillum Salomonis Off. - Piuttosto frequente nei luoghi montuosi presso Susa, nelle rupi di S. Martino ed ai Cenau. Radice fatua, mucilaginosa.

Per. Aprile, Maggio.

Sigillo di Salomone it. - P. commun fr.

La radice di questa specie, adoperata un giorno allo stato secco come risolvente, \& di sapore dolcigno a cui corrisponde l'aggettivo fatua del Re. 
P. multiflorum AlL. (Convallaria multiflora Re-Lin.). - Ovunque nelle selve.

Per. Maggio, Luglio.

Erba poligonata it. - P. multiflore fr.

\section{Gen. 4. - Comvallaria Lin.}

C. maialis LrN. - Lilii convallii fllores Off. - Nughet vern. - Nelle selve montane. Acre, antispasmodica.

Per. Maggio, Giugno.

Giglio delle convalli, Mughetto it. - Muguet de mai fr. Fuori uso in farmacia.

\section{GeN. 5. - Mayamthemam WiGG.}

M. bifolium DC. - Siti montani.

Per. Maggio, Giugno.

Gramigna di Parnaso it. - Mayanthème à deux feuilles fr.

GEN. 6. - Remseus LiN.

R. aculeatus Lin. - Ruscus o Bruscus Off. Intorno Susa e lungo il torrente di Foresto, nè manca a Condove e presso Caselette. Radice cor roborante diuretica.

Per. Aprile, Maggio.

Pungiratto it. - Fragon piquant, Houx-frelon fr.

Poco o niente usata nella farmacia di oggi.

B) Stili distinti.

GEN. 7. - Pris LiN.

P. quadrifolia Lis. - Volgare nelle selve ombrose dei monti.

Per. Maggio.

Erba Paris it. - Parisette à quatre feuilles fr. 


\section{Gen. 8. - Tamus LiN.}

\section{T. communis Lin. -- Nelle selve.}

Per. Aprile.

Tamaro it. - Tamier commun fr.

\section{Ordine CVI - Gigliacee (Liliaceae $D C$.)}

Sez. 11. ${ }^{2}$ - Fidice bulbosa : peduncoli non articolati.

A) Perigonio a sci pezzi liberi, o più o meno saldati fra loro solo alla base.

1) Stimma sessile tripartito.

\section{GEN: 1. - Tulipa Lin.}

T. sylvestris LiN. - Tulipan vern. - Fu da me in abbondanza raccolto nei monti di San Giorgio nel sito detto il Mariage andando verso li Gontè. Trovasi pure al Cenisio e nelle alpi di Exilles.

Per. Luglio.

Tulipano selvatico it. - Tulipe sauvage fr.

Colle delle Finestre passate le antiche fortificazioni verso il monte Pintas.

2) Stimma trifido provvisto di stilo.

\section{Gen. 2. - Erythroniuma Lin.}

E. dens canis Lis. - Dens canis Off. - In abbondanza lungo la via che conduce dai Magnolé a Frassinere. Farmaco disusato.

Per. Aprile, Maggio.

Dente di cane it. - Erythrone dent-de-chien fr. 
334

3) Stimma indiviso, provvisto di stilo trilobo, trigono, o semplice (nel Gen. Allium).

\section{Gen. 3. - Lilliam Lin.}

L. bulbiferum Lis. -- Nei pascoli declivi e specialmente nei prati del Collet e nei pascoli della Roccea a Condove.

$\beta$ croceum (Lilium croceum Chatx) - A Villarfocchiardo, da Ciantabue scendendo al Gravi (Bruno).

Per. Giugno, Luglio. (Fiori eretti campanulati).

Giglio rosso it. - Lis bulbifèrè fr.

L. martagon LiN. - Nelle selve ombrose e ne: pascoli álpini.

Per. Luglio, Agosto. (Fiori pendenti : pezzi del perigonio curvi all'insù).

Bella montanara, Martagone it. - L. Martagon fr.

Gen. 4. - Llaydia Salisb.

I. serotina SALISB. (Anthericum serotinum RE-LIN.). - Abbonda negli alti gioghi dei monti che sovrastano al Forte di Fenestrelle e sopra il Vallon, nè manca al Cenisio e nei monti di Giaveno.

Per. Giugno, Agosto. (Fiore bianco con tre strie rosee interne).

Falangio tardivo it. - Loïdie tardive fr.

Cenisio a Ronche (Negri).

\section{GeN. 5. - Gagea SAlisb.}

G. lutea Roexi e Schult. (Ornithogalum luteum Re $\beta$ Lis. - Frequente presso la fabbrica del marmo e nei prati del Cenisio.

Per. Maggio, Giugno.

Cipollaccio giallo it. - Gagée grisâtre fr. 
* G. Lioftardi Roem e Schult. - Cenisio (Bonjean in Bertol.).

Per. Giugno, Luglio.

Cipollaccio porrione it. - G. flstuleuse fr.

\section{Gen. 6. - Ormithogalum Liv.}

0. umbellatum Lin. - Comunissimo nei campi.

Per. Aprile, Maggio.

Aglio selvatico it. - Ornithogale en ombelle fr.

O. pyrenaicum Lin. - Nei campi a piedi del Colle di San Costanzo presso Susa, e frequente nei prati montani.

Per. Giugno, Luglio.

Coda di scoiattolo it. - 0 . des Pyrénées fr.

\section{GeN. 7. - Seilla LiN.}

S. bifolia Lin. - Nei prati umidi alpestri e presso le selve.

Per. Maggio.

Giacinto carico it. - Scille à deux feuilles fr.

\section{Gen. 8. - Hidurer LiN.}

* Stami semplici.

A. ursinum LrN. - Abbonda nei siti ombrosi.

Per. Maggio, Giugno.

Aglio orsino it. - Ail des ours fr.

A. acutangulum Schrad. (Allium angulosum RE-AlL.). - Raccolsi questa specie nelle acque stagnanti 
dei prati presso il ponte della Cenisia non lungi da Susa.

Per. Giugno, Luglio.

Aglio falsocalamo it. - A. anguleux fr.

Nel testo A. angulosum Lin., specie diversa, della Siberia.

A. fallax Don. (Allium senescens RE-Alu.) - Cenisio. Per. Luglio, Agosto.

Presso il lago del Cenisio (Negri).

A. douteux fr.

A. schoenoprasum LiN. $\beta$ alpinum DC. - Frequente nei prati alpini.

Per. Giugno. Luglio.

Porro sottile it. - A. civette fr.

Cenisio (Bouvier, Negri).

A. oleraceum Lis. (Allium carinatum Re non altri sec. Bertor.) - Nel Cenisio e presso Fenestrelle.

Per. Luglio.

Aglio di ortaggio it. - A. des lieux cultivés fr.

Nel testo Allium carinatum Lin., che è una varietà de!l'Allium olcraceum, la quale, secondo il Bertoloni e il Parlatore, non ancora $\dot{e}$ stata rinvenuta in Italia. Il Cesati (vedi la nota nel Compend. Fl. it., pag. 141), opina invece su dati molto convincenti che la pianta, dagli autori italiani data col nome di Linneo, sia veramente tale.

A. paniculatum Lin. - In siti sassosi e caldi.

Per. Luglio, Agosto.

Aglio rosellino it. - A. paniculé fr.

A. pallens L. $\beta$ paniculatum (Comp. Fl. it., Cesati, ecc.). 
* Stami, almeno i tre interni, col filetto terminato da tre punte, la mediana portante l'antera.

* A. descendens Lrs. - Musiné (Re, Fl. Tor.). Per. Giugno.

Aglietto, Aglio di serpe it. - A. des vignes fr. Allium sphaerocephalum Lin. $\beta$ Bertol.

A. sphaerocephalum LiN. - Siti sassosi del Cenisio. Per. Luglio, Agosto.

Aglietto, Aglio di serpe it. - A. à tête ronde fr. Sagra di San Michele.

\section{A. vineale Lis. - Frequente nei campi.}

Per. Giugno, Luglio.

Aglio pippolino it. - A. des vignes fr.

A questa specie deve riferirsi Allium arenarium Re-Lin. che il Re scrive trovarsi in gran copia alla Novalesa.

L'aglio dei nostri orti è Allium sativum Lin. La cipolla che presenta molte varietà è Allium cepa Lin. Il porro, Allium porrum Lin.

B) Perigonio di un sol peszo diviso solo brevemente nel lembo.

\section{Gex. 9. - Museari TourNF.}

M. comosum HilL. (Hyacinthus comosus RE-LiN.) In tutti i campi argillosi.

Per. Maggio, Giugno.

Giacinto delle vigne it. - Muscari à toupet fr.

\section{M. racemosum Mir.t. (Hyacinthus racemosus RE-LiN.)} - Comune nei pascoli e presso le mura delle vigne di Monpantero.

Per. Aprile, Maggio.

Giacinto muscino it. - M. à grappe fr. 
338

M. botryoides DC. Fl. fr. (Hyacinthus botryoides RELin.). - Trovato da Ig'nazio Molineri nel Musiné presso Caselette.

Per. Aprile, Maggio.

Muscini selvatici it. - M. raisin ir.

\section{Sez. S $^{2}$ - Eadici fibrose of fibroso-tubercolose : peduscoli articolati, fiori bianchi.}
A) Peduncoli articolati verso la metà della loro lunghez a.

GEN. 10. - Asplnodelus LiN.

A. albus Willd. (Asphodelus ramosus Re-Alz. dal sito). - A Monpantero nella contrada detta di San Marciano ed alle Blaccie.

Per. Maggio, Giugno.

Porraccio it. - Asphodèle blane fr.

Musiné (Re, Fl. tor., Vol. I, pag. 196, sotto A. albus Vedi la nota alla stessa pagina).

Nel testo A. ramosus Lin. dall'Allioni.

B) Peduncoli articolati verso la loro base.

GEN. 11. - Liliastram Tourne.

I. album Lisi. (Anthericum liliastrum RE-LIN.).

Prati del Collet: alpi di Frassinere, nel sito detto i Tulevit. Frequente pure nel Cenisio e nelle alpi di Giaveno.

Per. Giugno, Luglio.

Giglio di monte it. - Paradisia fleur de lis fr. 
Gen. 12. - Thalamginum Kunth.

Ph. liliago Schreb. (Anthericum liliago Re-Lin.). Abbonda nei pascoli aridi e declivi fra le vigne di Monpantero: nei prati di Fossimagna, $€$ generalmente in tutti i pascoli subalpini.

Per. Maggio, Giugno.

Liliagine it. - Phalangère fleur de lis $\mathrm{fr}$.

Ordine CVII - Colchicacee (Colchicacede DC:)

A) Radici bulbose.

Gen. 1. - Colehicum Lin.

C. autumnale Lrx. - Colchicum Off. - Fraidolina vern. - In tutti i prati. Rarlice acre, drastica, diuretica.

Per. Agosto, Ottobre.

Colchico it. - Colchique dautomne, Tue-chien fr.

La moderna terapia tiene ancora in buon conto i bulbi del colchico, e li usa nelle idropisie, nei reumatismi e nella gotta. Il loro principio attivo è chiamato Colchicina.

C. alpinum DC. (Colchicum montanum RE-ALL.). Frequente nei prati montani ed alpini.

Per. Luglio, Agosto.

Colchico alpino it. - C. des alpes fr.

Cenisio presso il lago, alla Ramasse (Parlat.).

Nel testo C. montanum Lin. dall'Allioni, specie diversa (Colchicum Bertolonii Stev.) di siti meridionali d'Italia. 


\section{GEN. 2. - IBulbocodinu LIN.}

B. vernum Lis. (Merendera bulbocodium RE-BALBIS). - Abbonda sul bosco nero e nel Cenisio sopra la posta.

Per. Giugno, Luglio.

Bulbocodio it. - Bulbocode printanier fr.

Nel testo Merendera bulbocodium Ramond, pianta diversa, dei Pirenei.

B) Radici fibrose.

\section{GEN. 3. - Verateum Lin.}

\section{V. album Lis. - Veratrum o Helleborus albus 0 ff.} - Vraro vern. - In tutti i prati alpini pingui e freddi. Velenoso.

Per. Giugno, Luglio.

Elleboro bianco: giglio verde: veladro it. - Vératre blane fr.

Il Veladro senza dubbio è velenoso.

\section{GEN. 4. - Tofieldia Huds.}

T. calyculata WAнLB. (Anthericum calyculatum RELIN.). - Siti alpestri umidi.

Per. Luglio, Agosto.

Falso asfodelo it. - Tofieldie à collerette fr.

Cenisio alla Gran Croce (Parlat.).

* E glacialis Parlat. (Tofieldia glacialis Gaud.) - Presso la Gran Croce con la specie.

* $\gamma$ ramosa Parlat. (Tofieldia glacialis $\beta$ ramosa Gaud.).

- Cenisio (Bonjean in Parlat.). 
* T. palustris Huds. - Presso il lago del Cenisio (Parlat.). (Negri sotto T. borealis Wahlb.).

Per. Luglio, Agosto.

Falso astodelo palustre it. - T. des marais fr.

Ordine CVIII - Giuncacee (Juncaceae Bartl.)

A) Capsula triloba uniloculare contenente tre semi.

Gen. 1. - Luzula DC.

- Fiori solitari.

* L. flavescens Gaud. - Cenisio alla Ramasse. (Parlat.).

Per. Giugno, Luglio.

Luxule jaunâtre fr.

* L. Forsteri DC. - Paludi di Campagnola verso Caselette (Re, App. 1 ad Fl. perl.).

Per. Maggio, Giugno.

Erba lucciola it. - L. des Forster fr.

L. pilosa Willd. (Juncus pilosus Re-Liv.). - Frequente nei pascoli e nelle selve: siti sterili della Roccea a Condove.

Per. Maggio, Giugno.

Lucciola sparpagliata it. - L. poilue fr.

* Fiori agglomerati a due o pil insieme.

* L. spadicea DC. - Altipiano del Cenisio (Negri): ad Eau blanche (Bouvier).

Per. Luglio, Agosto.

Lucciola rossigna it. - L. brune fr. 
* L. sylvatica Gaud. 6 Congesta Bouviek. - Altipiano del Cenisio (Bouvier).

Per. Maggio, Giugno.

Erba lucciolona it. - L. des bois fr.

L. lutea DC. (Juncus luteus RE-LiN. - Abbonda sul Cenisio e nelle alpi di Giaveno.

Per. Giugno, Luglio.

Bugnamacolo giallo it. - L. jaune fr.

L. campestris DC. (Juncus campestris RE-LiN.). Ovunque nei colli e nei pascoli.

Per. Maggio, Giugno.

Giunco secco it. - L. champêtre $\mathrm{fr}$.

* L. multiflora LEJ. - Altipianu del Cenisio (Negri).

${ }^{*}$ C. sudetica Bovvier (Juncus sudeticus Willd.). Cenisio (Bouvier).

Per. Giugno, Luglio.

L. multiflore fr.

L. spicata DC. (Juncus spicatus RE-LIN.). - Pascoli secchi alpini.

Per. Maggio, Giugno.

Bugnamacola nera it. - L. en epi fr.

L. nivea DC. (Juncus niveus RE-LIN.). - Volgare nelle selve montane.

Per. Giugno, Luglio.

Lucciola bianca it. - L. blanc de neige fr.

L. pediformis DC. (Juncus pediformis RE-VILL.). Frequente nei pascoli del Cenisio.

Per. Luglio, Agosto.

Busgnamacolo maggiore it. - L. en grappe fr. 
B) Capsula trigona triloculare a semi numerosi.

\section{Gen. 2. - Juneus Lin.}

* Culmo senza foglie.

J. filiformis Lin. - Paludi verso il piccolo Cenisio.

Per. Luglio, Agosto.

Giunco sottile it. - Jone filiforme fr.

J. conglomeratus Lis. - Ovunque in siti paludosi.

Per. Maggio, Luglio.

Giunco da mazzocchi it.

1. effusus Lis. - Ovunque nei pascoli umidi.

Per. Giugno.

Giunco dei contadini it. - J. commun fr.

- Culmi con foglie radicali e cauline.

* J. alpinus Vilz. - Presso il lago del Cenisio (Bouvier, Negri).

Per. Luglio, Agosto.

Giuncherello alpino it. - J. des alpes fr.

J. triglumis LrN. - Nei prati umidi delle sommità alpine.

Per. Luglio, Agosto.

Giuncherello nudo it. - J. ì trois glumes fr.

Cenisio alla Gran Croce e presso il lago (Parlat.) a Ronche. (Negri).

J. jacquinii LIN. - Pascoli alpini freddi.

Per. Luglio, Agosto.

Giunco a mazzetto it. - J. de Jacquin fr.

Cenisio a Ronche (Negri) presso la Ramasse (Parlat.)

Nel testo J. Jacquinii Willd. 
J. trifidus Lrs. - Frequente nelle alpi.

Per. Luglio, Agosto.

Giunco trifogliato it. - J. à trois pointes fr.

Cima di Ronche (Negri).

J. bufonius LrN. - Ovunque in siti umidi e ombrosi: frequente lungo le vie nei siti sterili di Condove al punto detto Bialèra della Reisia.

Per. Aprile, Maggio.

Giunco delle botte it. - J. des crapauds fr.

J. sylvaticus REICHARD. (Juncus articulatus RE-LiN.).

- In quasi tutti i siti umidi e paludosi.

Per. Giugno, Luglio.

Giunco selvatico it. - J. des bois fr.

J. compressus JACQ. (Juncus bulbosus RE-LIN.). Siti sub-umidi montani ai margini delle vie.

Per. Giugno, Luglio.

Giunco degli acquitrini it. - J. comprimé fr.

Ordine CIX - Ciperacee (Cyperaecue Juss.). Sez. $\|^{2}$ - Fiori ermafroditi : seaglie forahi distiche. Gen. 1. - Cyperas Lin.

- Tre stimmi : acheni triangolari.

C. fuscus Lin. - Siti umidi.

Ann. Luglio, Settembre.

Cipero nero it. - Souchet brun fr.

C. Iongus Lin. e Off. - Siti paludosi lungo i fossati. Radice aromatica.

Per. Luglio, Agosto.

Cipero it. - S. long fr. 
* Due stimmi : acheni compressi.

C. flavescens Lis. - Ovunque nei siti umidi. Ann. Giugno, Agosto.

Bottacino it. - S. jaunâtre fr.

Gen. 2. - Sehoenus Lin.

Sch. nigricans Lrv. - In tutti i pascoli montani umidi, presso Susa in siti analoghi alle Blaccie.

Per. Giugno, Luglio.

Giunco nero it. - Choin noirâtre fr.

Sez, $\mathfrak{2}^{2}-$ Fiori ermafroditi : seaglie fiorali embrieiate in molti versi.

Gen. 3. - Cladium R. Brown.

Cl. mariscus R. Brown. (Schoenus mariscus Re-LIN.). - Intorno ai laghi di Avigliana.

Per. Maggio, Giugno.

Serrago it. - Cladie marisque fr.

GeN. 4. - Pliøma PANZ.

Bl. compressus $\mathrm{P}_{\mathrm{ANZ}}$. (Schoenus compressus e Carez uliginosa Re-Lin.). - Pascoli umidi alpestri e montani, frequente intorno al lago del Cenisio: nelle selve umidette sopra la Novalesa.

Per. Giugno, Luglio.

Giunco moscarino it. - Scirpe comprimé fr.

Nei prati umidi della Sagra scendendo ai laghi di Avigliana (De Filippi). 


\section{Gex. 5. - Eleocharig R. Browr.}

E. palustris R. Browr. (Scirpus palustris Re-LiN.). - Presso le acque stagnanti : abbonda a Susa nei siti palustri di prato Donio presso la Brunetta.

Per. Maggio, Giugno. (Stilo bifido).

Giunco tondo it. - Héléocharis des marais $\mathrm{fr}$.

Laghi di Caselette.

E. acicularis R. Brow. (Scirpus acicularis RE-LIN.). - Nelle acque stagnanti.

Per. Giugno. (Stilo trifido).

Capitelli it. - H. épingle fr.

\section{Gen. 6. - elrpars Lin.}

' Spighette solitarie terminali del culmo, o dei rami.

1) Guaine sprovviste di lamina fogliacea.

* S. parvulus Rowar. e Schllt. - Rive del lago grande di Caselette. (Gay in Parlat., Fl. it.).

Ann. Giugno, Luglio.

Giuncolino nano it. - Scirpe nain fr.

* S. pauciflorus Lightr. - Altipiano del Cenisio. (Negri).

Per. Giugno, Luglio.

S. pauciflore $\mathrm{fr}$.

2) Gnaine, almeno la superiore, provriste di lamina fogliacea.

S. caespitosus Lrx. - Prati umirletti e freddi delle alpi. Abbonda sulle rive del lago del Cenisio.

Per. Giugno, Agosto.

Giuncolino giallo it. - S. en gazon $\mathrm{fr}$.

Località medesima sul Cenisio. (Bouvier). 
* S. alpinus Schleich. - Siti elevati ed umidi del Cenisio. (Reut. in Parlat.). Altipiano del Ce. nisio. (Negri). Rive del lago. (Bouvier).

Per. Luglio, Agosto.

Giuncolino d'alpe it. - S: des alpes fr.

* Spighette laterali, gemine o parecchie in ombrelle.

S. setaceus LrN. - Rive della Dora in siti umidi. Ann. Giugno, Luglio. Giuncolino setaceo it. - S. sétacé fr.

S. supinus Lrs. - Pascoli sabbiosi presso la Dora, tra Bussolino e Foresto. Vedi Balbis, Elenc., pag. 84. Ann. Luglio, Settembre.

Giunco giacente it. - S. couchẻ fr. Nel testo S. supinus Willd.

S. holoschoenus Lin. - Frequente nei siti umidi. Per. Giugno, Settembre.

Giunco sottile it. - S. jonc fr.

*. Spighette in ombrelle terminali.

S. maritimus LIN. - Siti umidi lungo la Dor'a. Per. Maggio, Luglio.

Erba nocea it. - S. maritime fr.

Gen. 7. - Phymelhospora VAHL.

* R. alba VAhL. - Alle falde del Musiné (Re, Fl. Tor.).

Per. Luglio, Agosto.

Giunco tenero it. - Rhynchospora blane fi. 


\section{GEN. 8. - Eriophorum LIN.}

" Spighetta terminale solitaria eretta.

E. vaginatum LiN. - Presso le acque nelle alpi, e nelle sommità fredde del Cenisio.

Per. Luglio, Agosto.

Giunco sericeo it. - Linaigrette engainée fr.

* E. Scheuchzeri Hoppe. - Cenisio ad Fau blanche (Verlot.). Cenisio (Bertero, Colla).

Per. Luglio, Agosto.

Pennacchio rotondo it. - L. capitée fr.

* Spighette 3-molte pendenti alla maturita.

E. latifolium Hoppe. ( Eriophorum polystachyon $\mathrm{RE}$ $\beta$ LIN. - E. polystachyon ALL. . - Ovunque nei prati umidi alpini.

Per. Giugno, Luglio.

Pennacchi penzoli it. - L. à pédoncules rudes fr.

* E. gracile Koch. - Cenisio (Bonjean in Bertol.).

Per. Giugno, Luglio.

Pennacchio coda di volpe it. - L. d̀ pédoncules pube. scents fr.

Sez. $3^{2}$ - Fiori per lo piu monoici, raramente dioiei.

Gen. 9. - Elyma Schrad.

E. spicata Schrad. (Carex Bellardi Re-All.). Ovunque nei pascoli del Cenisio.

Per. Giugno, Luglio.

Carotto sottile it. - Elyne en épi fr. 
GEN. 10. - Kobrosia WILLD.

k. caricina Wilid. (Carex bipartita Re-All. ?). - Frequente intorno al lago del Cenisio.

Per. Luglio, Agosto.

Carotto vuginoso it. - Kobrésie carex fr.

Abbiamo messo con dubbio il sinonimo, poichè il Parlatore opina che la Carex bipartita All. debba piuttosto riferirsi a Carex approximata Hoppe che a questa specie, alla qualc molti autori, tra i quali il Colla e il Bertoloni, l'hanno rapportata (Parlat., Fl. it. Vol. 2, pag. 142.).

\section{Gen. 11. - Carex Lin.}

Sez. $a^{2}$ - Spiga terminale semplice solitaria dioica, o androgina (monoica).

1) Stilo bifido.

C. dioica Lrx. - Intorno al lago del Cenisio, e nei siti palustri di Coldemozzo.

Per. Maggio. (Dioica).

Carotto dritto it. - Carex diö́que fr.

${ }^{*}$ C. davalliana Smтth. - Siti montani palustri di Bussolino. ( Re, App. III ad. Fl. Ped.). Cenisio. (Bertero in Colla Herb.).

Per. Maggio. (Dioica).

Carotto falcato it. - C. de Davall. fr.

Presso il lago del Cenisio. (Negri).

2) Stilo trifido.

C. rupestris BeLL. - Fu trovata nelle rupi del Cenisio da Ignazio Molineri.

Per. Luglio, Agosto. (Androgina).

Carice rupina it. - C. des rochers fr. 
Nel testo C. rupestris All. sotto il di cui nome è riportata da quasi tutti gli autori, salvo dal Compendio della Flora ital. del Cesati, in cui troviamo la seguente nota:

"Fin qui le tre specie di C. rupestris, C. bicolor, C. nigra, furono attribuite all Allioni per una falsa interpretazione del segno $\div$ apposto nella Flora pedemontana, cio che il prof. Cesati si riserva di dimostrare in apposita dissertazione unche rispettivamente ad altra specie di quella Flora."

C. pauciflora LightF. - Intorno al lago del Cenisio.

Per. Giugno, Luglio. (Androgina).

Carotto lesinino it. - C. pauciflora fr.

Nel testo C. pauciflora Schk.

* C. microglochin Wahlesb. - Presso il piccolo lago del Cenisio. (Bonjean in Bertol., Balbis in Bertol.).

Per. Luglio, Agosto.

Carotto aristato it. - C. à utricules subulés fr.

\section{Sez. $\$^{2}-$ Spiga terminale}

conposta e formata di spighette androgine.

Stilo bifido.

C. foetida ALL. - Sui gioghi più alti e freddi del Cenisio ed all'Assietta.

Per. Luglio, Agosto.

Carice puzzolente it. - C. fétide fr.

C. incurva LightF. (CareX juncifolia RE-ALL.). - Siti umidi e freddi del Cenisio: rara.

Per. Giugno, Luglio.

Carice inarcata it. - C. courbé fr.

Dintorni del lago del Cenisio. (Negri). 
C approximata Horpe. (Carex bipartita RE-ALL., sec. Parlat.). - Frequente presso il lago del Cenisio.

Per. Luglio, Agosto.

Carice piè di lepre it. - C. ì épillets rapprochés fr.

C. brizoides Lin. - Siti erbosi incolti.

Per. Maggio, Giugno.

Carice brizolina it. - C. brize fr.

C. vulpina Lin. - Nei siti acquosi.

Per. Maggio, Giugno.

Carice compatta it. - C. jaunâtre fr.

* C. divulsa Good. - Dimenticata dal ch. Re nella Fl. di Susa, ma trovata dal ch. Avogradro in siti erbosi presso Susa. (Colla Herb., T. VII., p. 42.8).

Per. Maggio, Giugno.

Carice diradata it. -- C. écarté fr.

C. paniculata Lin. - Siti palustri del Cenisio, e in abbondanza presso Susa, nei prati di Croale.

Per. Maggio, Luglio.

Carice pannocchiuta it. - C. paniculé fr.

Presso il lago del Cenisio. (Negri).

C. remota Lrs. - Ovunque in siti umidi ed ornbrosi.

Per. Maggio, Giugno.

Carice ascellare it. - C. espace fr.

C. canescens Lin. - Nel Cenisio.

Per. Giugno, Luglio.

Carice cenericcia it. - C. blanchâtre fr. 
C. echinata Murr. Prod. (Carex stellulata Re-Willd.). - Siti paludosi, montani e submontani.

Per. Maggio, Giugno.

Giunco stellare it. - C. étoilé fr.

C. Ieporina Lrs. - Orunque nei siti umidetti. Per. Giugno, Luglio.

Carice ovata it. - C. des lièvres fr.

" stilo trifido.

C. curvula Ali. (Carex tripartita Re-AlL., sec. ParLATORE). - Comune nelle alpi.

Per. Luglio, Agosto.

Carice giuncolina it. - C. à feuilles courbées fr.

Dintorni del lago del Cenisio. (Negri).

\$ez. $3^{2}-$ piga terminale andiregina, maschile alla base. fenminile sll'apice, tutte le altre fenminili.

- Stilo bifido.

C. bicolor Bell. - Carex androgyna Balbis. - Nei siti freddi ed umidi del Genisio presso i ruscelli: rara.

Per. Luglio, Agosto.

Carice bianco-porporina it. - C. bicolore fr.

Dintorni del lago del Cenisio. (Negri).

- Stilo trifido.

C. nigra BeLL. - Nei pascoli alti umidetti del Cenisio.

Per. Luglio, Agosto.

Carice nereggiante it. - C. noir fr.

C. bicolor. e nigra All., nel testo, e negli altri autori. (V. la nota a pag. 350).

C. atrata Lis. - Cenisio: alpi di Giaveno.

Per. Luglio, Agosto.

Carice abbronzata it. - C. en deuil fr. 
Sez. 4" - Spighe di sesso distinto, una terminale maschile, due o tre superiori maschlli : le altre femminili; raramente maschili all'apice.

- Otricello fruttifero senza rostro o brevemente rostrato: stilo trifido.

C. montana Lin. Fl. suec. - $\beta$ procerior Gaud. Kock. Parlat. Carex conglobata Re-All.). - Raccolta dal ch. Allioni nell' ascendere all' Assietta.

Per. Maggio, Giugno.

Carice villosa it. - C. de montagne fr.

C. ericetorum Polich. - ( Carex approximata REALL.). - Cenisio.

Per. Luglio, Agosto.

Carice cigliata it. - C. des bruyères fr.

Cenisio a Patta Creusa (Bonjean in Parlat.).

Cirna d'Eeau blanche. (Negri).

* $\beta$ membranacea Koch. - (Carex membranacea Hoppe). - Collina presso le rive del lago del Cenisio. (Bouvier).

C. praecox JACQ. - In tutti i pascoli.

Per. Aprile, Maggio.

Carice primaticcia it. - C. précoce fr.

Nel testo Carex praecox All.

c. humilis Leyss. (Carex prostrata Re-ALL.). - Frequente ovunque nei pascoli montani.

Per. Aprile, Maggio.

Carice rossigna it. - C. bas fr.

C. digitata Lin. - Siti montani e sub-montani. Per. Maggio.

Carice digitata it. - C. digité fr. 
C. pilosa Scop. - Ovunque nelle selve montane. Per. Maggio.

Carice pelosa it. - C. poilu fr.

Nel testo Carex pilosa Vitm.

C. panicea Lrs. - Volgare nei siti umidi mon$\operatorname{tani}$ e sub-montani.

Per. Maggio, Giugno.

Carice migliacea it. - C. panic fr.

Dintorni del lago del Cenisio. (Negri).

* C. nitida Host. - Cenisio (Balbis, Elench.). Pascoli sterili e soleggiati tra Alpignano e Rivoli (Re, Fl. Tor.).

Per. Maggio, Giugno.

Carice lustra it. - C. luisant fr.

C. pallescens Lis. - Frequente ovunque nei prati e nei pascoli umidetti.

Per. Giugno, Luglio.

Carice verde pallida it. - C. pâle fr.

* C. capillaris Lis. - Cima di Ronche (Negri) : Cenisio (Bouvier, Bonjean in Colla e Colla).

Per. Giugno, Luglio.

Carice capillare it. - C. capillaire fr.

C. limosa Lix. --- Presso il lago del Cenisio. Per. Giugno, Luglio.

Carice fanghereccia it. - C. des fanges $\mathrm{fr}$.

G. vulgaris Fries. (Carex ceespitosa RE-ALL.). Intorno ai laghi di Avigliana e del Cenisio.

Per. Maggio, Giugno.

Carice flagellosa it. - C. commun fr. 
* C. ornithopoda Willd. - Cenisio, presso il lago nero, e rive meridionali del grande. (Verlot.). Per. Maggio, Giugno.

Carice piè d' uccello it. - C. pied-d'oiseau fr.

C. acuta Lin. - Lesca vern. - Comunissima presso le acque.

Per. Maggio.

Nocea it. - C. aigu fr.

c. stricta Good. (Carex elata Re-All.). - Siti paludosi della valle d' Oulx.

Per. Maggio.

Carice filacciosa it. - C. roide fr.

Troviamo ben due volte, per errore materiale, dall'autore segnata tra le Carici, Carex filiformis Lin., e sempre con la nota crescente in abbondanza (ubertim communis), intorno al lago del Cenisio. Or qui trattasi non già di Carex filiformis Lin., che non ancora è stata trovata per quanto sappiamo in Piemonte, ma di Carex filiformis All. che il Parlatore (Fl. it., T. II, pag. 219), rapporta a Carex tomentosa Lin. Mant., come già anteriormente l'avea riferita il Pollini. (Fl. veron., T. III, pag. 74).

* Otricelli fruttiferi pit o meno lungamente rostrati : stilo bifido in Carex mucronata, trifido nelle altre specie.

C. mucronata ALL. - Alpi di Bardonêche, e nel Cenisio verso la Novalesa nelle fenditure delle roccie.

Per. Giugno, Agosto.

Carice setacea it. - C. mucroné fr.

C. frigida ALL. - Frequente intorno al lago del Cenisio e i ruscelli freddi.

Per. Luglio, Agosto.

Carice divergente it. - C. des frimas fr. 
C. sempervirens VILL. (Carex saxatilis Re-Ar.t, non Lin. sec. Bertol.). - Nel Cenisio e nei monti più elevati della valle di Bardonêche.

Per. Giugno, Luglio.

Carice moltiforme it. - C. toujours vert fr.

Carex ferruginea Schrk.

C. Ilava Lin. - Ovunque nei siti incolti.

Per. Maggio, Giugno.

Carice rimbeccata it. - C. jaune fr.

o. polystachya Gaud. (Spighette terminali 5-6). (Carex foliosa RE-ALL.). - Siti paludosi alle falde dell'Assietta.

(Vedi Colla Herb. T. V, pag. 509).

${ }^{\star}$ C. ferruginea Scop. - Cenisio. (Balbis in Bertol.). Piccolo Cenisio (Bouvier).

Per. Giugno, Luglio.

Carice dimagrata it. - C. ferrugineux fr.

C. distans Lin. - Ovunque nei pascoli umidetti. Per. Maggio.

Panicastrella acquatica it. - C. distant fr.

¿. sylvatica Huds. - (Carex patula Re-Scop.). Selve montane in siti umidetti.

Per. Maggio.

Carice silvana it. - C. des bois fr.

Nel testo Carex patula Lin., crediamo per errore materiale.

* C. firma Host. - Cenisio ad Eau blanche (Balbis Misc. alt.).

Per. Luglio, Agosto.

Carice rigida it. - C. ferme fr. 
C. vesicaria Lrs. - Paludi di Caselette, intorno al lago del Cenisio ed altri simili siti.

Per. Míaggio, Giugno.

Carice vescicosa it. - C. en vessie fr.

C. hirta Lin. - Nei prati erbosi declivi non umidi.

Per. Maggio, Giugno.

Carice villosa it. - C. hérissé fr.

* C. ampullacea Good. - Dintorni del lago del Cenisio. (Balbis, Misc. bot. alt.). (Parlat.), (Negri).

Per. Giugno, Luglio.

Carice ampollacea it. - C. ampoulé fr.

* C. fulva Good. - C. Hornschuchiana Hoppe. - Dintorni del lagro del Cenisio. (Verlot).

Per. Maggio, Giugno.

Carice lionata is. - C. de Hornschuch fr.

Ordine CX - Graminacee (Gramineae Juss.)

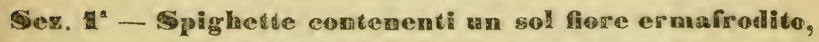
acconpagarto o mon dis mozziconi di fiori rudinentali, raranente (nel Gen. 䣶ordeum) talune delle spighette contenenti invere talvolta an fiore maschile o mextro.

- Una, o tre spighette (Gen. Hordeum) inserite su denti della rachide e addossate a rispettive incervature della stessa.

\section{Gen. 1. - Nardus Lin.}

N. stricta Lin. - Pascoli e prati montani.

Per. Giugno, Luglio. (Stilo, e stimma unico).

Fieno di monte it. - Nard roide fr. 


\section{Gen. 2. - Psillarug Trin.}

P. nardoides Trin. (Nardus aristata RE-LiN.). Pascoli macilenti di Foresto.

Ann. Maggio.

Erba setolina it. - Psilure faux-nard fr.

\section{Gen. 3. - Hordean Lin.}

H. murinum Lin. - Ovunque presso le vie e le case.

Ann. Giugno, Luglio. (Spighette laterali maschili o neutre). Erba codola it. - Orge queue-de-sonris fr.

* H. vulgare Lin. - Coltivato.

Ann. Maggio.

Orzo it. - Ordi vern. - O. commune fr.

* Spighette-mai inserite su denti delle rachide, nè addossate ad incavature della stessa.

\section{Gen. 4. - Cymodom Rich.}

C. dactylon Pers. (Panicum dactylon Re-LiN.). Gramen officinarum - Gramon vern. - Nei pascoli e nei campi ovunque. Radice refrigerante la quale sostituisce la radice dell'Agropyrum repens.

Gramigna it. - Chiendent commun fr.

Il rizoma del Cynodon dactylon fu molto commendato un giorno in decotto e in altre forme come antiflogistico purgativo del sangue, ecc.: oggi il suo uso è di molto scemato. 


\section{Gen. 5. - Alopecurus LiN.}

- Glume mutiche: una sola glumetta.

A. pratensis LiN. - Frequente nei prati umidi.

Per. Maggio, Giugno.

Coda di volpe it. - Vulpin des prés fr.

Buon pascolo, sia fresco, sia allo stato di fieno.

A. agrestis Lin. - Comunissimo nei campi.

Ann. Maggio.

Erba topina it. - V. des champs fr.

Pascolo eccellente fresco e secco.

A. geniculatus Lin. - Nei siti acquosi.

Per. Aprile, Maggio.

Codino aquatico it. - V. genouillé fr.

* Glume aristate: due glumette.

A. Gerardi Vill. (Phleum Gerardi Re-All.). - Prati elevati del Cenisio.

Per. Luglio, Agosto.

Capolino bianco it. - V. de Gérard fr.

Gen. 6. - Deyenxia Palis de Beauv.

D. sylvatica Kunth. (Agrosłis arundinacea Re-Lin.). - Siti sassosi alpestri del Cenisio.

Per. Giugno, Agosto.

Cannella di selva it. - Deyeuxie des bois fr.

\section{GeN. 7. - Callamogrostis Adans.}

G. epigejos Rотн. (Arundo epigejos Re-Lin.). - Nei siti sabbiosi.

Per. Giugno, Luglio.

Calamo scrittorio it. - Calamagrostide commune fr. 
360

\section{Gen. 8. - Lasiagroostis Link.}

L. calamagrostis Link. (Agrostis calamagrostis RELin.) - Siti caldi e sassosi montani, specialmente presso i torrenti.

Per. Giugno, Agosto.

Cannella ingannavena it. - Lasiagrostide calamagro. stide fr.

Siti alpestri da Susa al Cenisio (Negri).

\section{Gen. 9. - grostis Kunth.}

- Gluma inferiore piu grande della superiore: due glumette.

A. alba Lin. - Siti sterili di Condove alla Grangietta.

Per. Giugno, Agosto.

Capellini it. - Agrostide blanche fr.

Questa specie è variabilissima, ed è stata divisa e suddivisa in gran numero di altre specie, considerate oggi giustamente corne altrettante forme dipendenti dalle località diverse.

* A. vulgaris Wither. - Cenisio (Bonjean in Parlat.) e altrove in siti così di pianura che di montagna.

* $\gamma$ pumilaParlat. - (Agrostis pumila Lin.). - Siti umidi di Caselette (Balbis addit. ad Fl. Ped. sotto Agrostis intermedia).

Per. Luglio, Agosto.

Capellini it. - A. vulgaire fr.

A. stolonifera Lis. - Ovunque in siti umidi: abbonda nei prati di Venaus.

Per. Giugno, Luglio. Miglio d'acquitrino it. - A. verticillée fr. Agrostis verticillata Vill. 
- Gluma inferiore pit grande della superiore: uua glumetta.

A. canina Lin. - Siti umidi presso la Dora.

$\beta$ mutica Parlat. (Agrostis capillaris Re-AlL. non LIN.). - Nei prati umidi.

Per. Giugno, Luglio.

Capellini it. - Plon vern. - A. des chiens fr.

Agrostis rubra Re non è che una forma poco stabile della canina, con la quale coabita. Malamente dall'autore fu creduta Agrostis rubra Lin. la quale è specie diversa affatto, della Svezia e della Lapponia (Parlat., Fl. it., vol. I, pag. 187).

* A. alpina Scop. - Ad Eau blanche sul Cenisio (Bouvier).

$\beta$ flavescens (Avena auraîa Re-Alu.). - Nei pascoli più elevati del Cenisio.

Per. Luglio, Agosto.

Capellini dorati d'alpe it. - A. des alpes fr.

A rupestris Alt. - Nel Cenisio.

Per. Luglio, Agosto.

Capellini violetti it. - A. des rochers fr.

Ad Eau blanche (Bouvier).

- Gluma inferiore pilu piccola della superiore: due glumette.

A. spica venti LiN. - Comunissima nei campi.

Ann. Maggio, Giugno.

Spica venti it. - Lionza, bianchet vern. - A. jouet du vent $\mathrm{fr}$.

Tutte le specie di Agrostis sono appetite dal bestiame specialmente A. vulgaris, canina e alpina. 
362

\section{Gen. 10. - Stipa Lin.}

S. pennata Lin. - Siti aprici delle Blaccie, e sassosi del Musiné.

Per. Luglio, Agosto.

Lino delle fate it. - Stipe plumeux fr.

S. capillata Lin. - Presso Susa, e nelle rupi di S. Michele della Chiusa.

Per. Giugno, Luglio.

Stuzzichella crespa it. -- S. chevelue fr.

Gen. 11. - Bryza AL. Brown

0. clandestina AL. Brown. (Phalaris orizoides RELin.). - Presso le acque.

Per. Luglio, Agosto.

Asperella it. - Leersie à fleurs de riz fr.

Leersia oryzoides Swartz.

\section{Gen. 12. - Milium Lin.}

M. effusum Lin. - Selve montane ed alpine.

Per. Maggio, Luglio.

Gramigna cedrata it. - Millet étalé fr.

\section{Gen, 13. - Plo ala a is IAN.}

Ph. arundinacea Lin. - Ai margini dei fossi. Per. Giugno, Luglio. Scagliola cannella it. - Phalaris bigarrée fr. 


\section{Gen. 14. - Phleum Lin.}

- Spighette senza mozzicone di fiore rudimentale.

Ph. alpinum Lin. - Frequente nelle alpi.

Per. Giugno, Luglio.

Codina violetta it. - Phléole des alpes 1r.

Altipiano del Cenisio. (Negri).

A questa specie deve rapportarsi Phleum geniculatum Bell. che il Re scrive trovarsi nei siti sterili del Cenisio. (Colla, Herb., T. VI, pag. 159).

Ph. pratense Lin. - Orunque nei prati.

$\beta$ nodosum Gavd. (Ph. nodosum Re-Lin.). - Presso i campi e nei pascoli montani.

Per. Maggio, Giugno.

Codolina it. - P. des prés fr.

È molto gradito al bestiame.

* Spighette con rudimento di un secondo fiore.

Ph. asperum $J_{A C Q}$. (Ph. viride RE-AlL.). - Campi sabbiosi.

Ann. Aprile, Maggio.

Fleo ramoso it. - P. rude fr.

Nel testo $\mathrm{Ph}$. viride Bell.

Ph. Michelii All. - Trovato da Ignazio Molineri sul Musiné.

Per. Maggio, Giugno.

Codolina it. - P. de Micheli fr.

Nelle vigne di Caselette (Re, Fl. Tor.).

Le specie di Phleum sono mangiate con piacere dal bestiame. 
Sez. $\Im^{\mathrm{a}}-\$$ pighette contenenti un salo figre ermafrodito accompagnato da uno o due flori maschili, ovvero da una of da due glumette di fiori mentri.

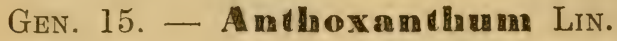

A. odoratum Lin. - Ovunque nei prati.

Per. Maggio, Giugno.

Paleino odoroso it. - Flouve odorante fr.

Le radici e le foglie comunicano al fieno un gratissimo odore.

\section{Gen. 16. - Holeus LiN.}

H. Ianatus Lrs. -- In tutti i prati.

Per. Maggio, Giugno.

Fieno bianco it. - Houlque laineuse fr.

H. mollis Lin. - Intorno ai campi montani.

Per. Giugno, Luglio.

Fieno canino it. - H. molle fr.

Gen. 17. - Setaria Palis de Beauv.

S. verticillata Palis de Beauv. (Panicum verticillatum Re-Lin.). - Abbonda nei campi.

Ann. Luglio, Agosto.

Fieno stellino it. - Sétaire verticillée fr.

S. viridis Palis de Beauv. (Panicum viride Re-Lin.). - Baraval vern. - Nei campi e nei prati.

Ann. Luglio, Agosto.

Panico falso it. - S. verte fr. 


\section{GEN. 18. - Tragug Desf.}

T. racemosus Desf. (Cenchrus racemosus RE-LiN.). - Ovunque nei pascoli umidi.

Ann. Giugno, Luglio.

Gramigna lappola it. - Bardanette en grappe fr.

\section{GEN. 19. -}

P. crus-galli Lrs. - Ovunque nei prati umidi e nei campi pingui.

Ann. Luglio, Agosto.

Panico selvatico it. - Panic pied-de-coq fr.

Buon pascolo.

Il miglio, mei vern., originario delle Indie orientali che è coltivato per i suoi semi, onde cibare uccelli di gabbia e domestici, è Panicum miliaceum Lin.

Gen. 20. - Oplismeras Palis de Beauv.

0. undulatifolius Roem e Schult. (Panicum Burmanni Re-Balbis). - Nei pascoli delle selve ombrose, frequente nei boschi di Condove lungo la Dora.

Ann. Luglio, Agosto.

Raspellone irsuto it. - Oplismène à feuilles ondulées $\mathrm{fr}$. Nel testo Panicum Burmanni, Wild.

\section{GEN. 21. - Digitaria Scop.}

D. sanguinalis Scop. (Panicum sanguinale RE-LIN.). - Abbonda nei campi.

Ann. Giugno, Luglio.

Sanguinella it. - Digitaire sanguinole, manne terrestre fr. 
366

GEN. 22. - Arrilnengutherum PALIS DE BEAUV.

A. elatior Mert, e Kock. (Avena elatior RE-Lin.). In tutti i prati.

Per. Maggio, Luglio.

Vena maggiore it. - Arrhénathère avoine fr.

Ottimo pascolo, fresco e secco, per le bovine e pei cavalli.

Sez. $3^{2}-$ Spighette contenenti due o piờ fiori ermafrediti, accompagmati o non da fori maschali neutri - rudimentali.

- Una o pit spighette sessili o quasi inserite sopra denti della rachide, e addossate a rispettive iucavature della stessa.

Gen. 23. - Brachellapodian Palis de Beauv.

B. pinnatum P. DE BeAuv. -- (Bromus pinnatus REAlu.). - Abbonda nei prati e nei pascoli macilenti.

Per. Giugno, Luglio.

Palèo it. - Brachypode corniculé fr.

B. sylvaticum Roem e Schult. (Bromus dumosus ReVill.). - Ovunque nei dumeti.

Per. Maggio, Giugno.

Palèo peloso it. - B. des forêt fr.

* Gen. 24. - Seande Lin.

S. cereale Lin. - Coltivata.

Ann. Maggio.

Segale it. - Sceil vern. - Seigle cultivé fr.

La Segale è il prezioso cereale dei siti elevati e freddi delle alpi : essa, per tutta la durata della sua vegetazione, assorbe la somma di 1900 gradi di calore, nel mentre il frumento comune ne richiede 2:39 (Cantoni), e daltronde la sua composizione poco differisce da quella del frumento. 


\section{* Gen. 25. - Triticum Lin.}

\section{T. vulgare VILL. - Coltivato.}

Ann. Maggio, Giugno.

Frumento it. - Gran, frument, vern. - Frument commun francese.

La cultura del frumento rimonta nell' antico continente alle originistesse dell' agricoltura. Nella China era coltivato 2822 anni prima dell' êra volgare: del suo luogo rativo primitivo si sa poco o nulla. Le specie e le varietá di frumento possono dividersi in due gruppi: frumenti teneri, frumenti da pane nei quali le sostance amilacee preponderano sul glutine, e frumenti duri, grani duri di Sicilia, di Puglia, di Tangarok, grani da far paste, maccheroni, ecc., nei quali viceversa, il glutine predomina.

\section{GEN. 26. - egilops Lin.}

A. ovata Lin. - Pascoli sterili a Susa, e sopra Foresto.

Ann. Maggio.

Gramigna stellata it. - Egilope ovale fr.

Gen. 27. - Amopremang Palis de Beauv.

A. caninum Roem e Schult. - (Elymus caninus RELin.). - Pascoli e dumeti.

Per. Maggio.

Grano cattivo it. - Agropyre des chiens fr.

A. repens Palis. de Beauv (Triticum repens Re-Lin.). - Triticum caninum o Gramen Off. - Luugo i campi e le siepi. Radice refrigerante.

De te canino it. - A. rampant fr.

Per la virtù medicinale della specie ci riportiamo a quanto abbiamo scritto a pag. 35็ sotto a Cyuodon dactylon. 


\section{Gen. 28. - Loliam LIN.}

L. perenne Lin. - Comunissimo nei prati e nei pascoli.

Per. Giugno, Agosto.

Gioglio selvatico it. -- Ivraie vivace fr.

L. temulentum Lin. - Nei campi.

Ann. Giugno, Luglio.

Loglio, gioglio cattivo it. - - I. enivrante fr.

I semi di questa specie sono velenosi : producono coliche, delirio, e, in grandi dosi, la morte: essi, quando sono mescolati ai granelli del frumento, e con questi macinati, rendono il pane malsano.

Gen, 29. - Namelarems Buuff. e Fing.

N. tenelius Rerchis. (Triticum tenellum RE-LiN.). Siti sabbiosi presso i torrenti.

$\beta$ unilateralis (Triticum biunciale Re-VILL.). Raccolto nei pascoli aridi di Foresto e lungo i campi da Ignazio Molineri.

Ann. Maggio, Giugno.

Grano paleino it. - Nardure délicat fr.

- Spighette mai inserite su denti del'a rachide, nè addossate ad incavatura della stessa.

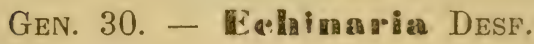

E. capitata Dess. (Cenchrus capitatus RE-LrN.). Sopra Foresto, e nei siti incolti intorno alle vigne di Monpantero.

Ann. Maggio, Giugno.

Gramigna calcotreppola it. - Echinaire en tête fr. 
Gen. 31. - Mellen Lin.

M. Magnolii Gren. e Godr. (Melica ciliata Re-Vill. non Lin. sec. Gren. e Godr.). - Frequente nei siti sassosi montani: qua e là nelle mura delle vigne di Monpantero.

Per. Aprile, Maggio. (Glumetta inferiore barbata).

Gramigna barbata it. - Mélique ciliée fr.

M. nutans Lin. - Nelle selve : presso Susa nella contrada detta di Croale.

Per. Maggio, Giugno. (Glumetta inferiore imberbe).

Melica penzola it. - M. penchée fr.

M. uniflora Retz. (Melica Lobelii Re-VILL.). - Nelle selve: presso Susa nella contrada delle Gorgie.

Per. Maggio, Giugno. (Glumetta inferiore imberbe).

Vena rossa it. - M. uniflore fr.

Gen. 32. - Deschampsia PaLis de Beauv.

D. coespitosa P. de Beauv. (Aira coespitosa Re-Lin.). - Nei prati umidetti lungo la Dora: e presso Codizar sopra Chiomonte.

Per. Luglio, Agosto.

Migliarino it. - Deschampsie en gazon fr.

Specie variabile a seconda dei luoghi, specialmente per la statura, la quale nei siti alpini è molto più bassa che in pianura.

D. flexuosa Trin. (Aira montana Re-All.). - Frequentissima nei monti di Giaveno e sul Cenisio.

Per. Giugno, Agosto.

Panico capellino it. - D. flexueuse fr.

Aira flexuosa Lin. Avenella flexuosa Parlat.

Nel testo A. montana Lin., specie diversa non italiana. 


\section{Gen. 33. - Plhagmites Trin.}

Ph. communis Trin. (Arundo phragmites RE-LIN.). Ovunque in siti palustri.

Per. Luglio, Agosto.

Canna di palude it. - Roseau commun fr. Appetita dalle bovine.

\section{Gen. 34. - A irm LIN.}

A. caryophyllea LiN. -- Ovunque nei pascoli sterili.

Ann. Maggio, Giugno.

Pabbio it. - Canche caryophyllée fr.

\section{Gen. 35. - Serleria Scop.}

- Glumetta inferiore 3-5 dentato-aristata, o dentato-mucronata.

S. coerulea Arduin. (Cynosurus coeruleus Re-LiN.). ...- In tutti i pascoli montani ed alpestri.

Per. Giugno, Luglio.

Codino azzurro it. -- Seslérie bleuâtre fr.

Altipiano del Cenisio (Negri).

S. argentea SAv1. (Cynosurus cylindricus Re BALBIS). - Trovai in abbondanza questa rara specie nei dirupi della cateratta di Foresto, e mi ebbi la certezza che la stessa fosse il Cynosurus cylindricus dallo stesso ch. prof. Balbis, al quale comunicai gli esemplari.

Per. Giugno, Luglio.

Sesleria argentina it. - S. argentée fr. 
* S. sphaerocephala Arduin. - A Ronche sul Cenisio (Bouvier). Località medesima (Bellardi, App. ad. Fl. ped.).

Per. Giugno, Luglio.

Codino capolino it. - $\mathrm{S}$. à tête ronde $\mathrm{fr}$.

** Glumetta inferiore intera.

S. disticha Pers. (Poa disticha Re-JACQ. Poa seslerioides ALL.). - Ignazio Molineri osservò che questa specie nasce in abbondanza nei monti di Giaveno presso il colle della Russa.

Per. Luglio, Agosto.

Fienarola cerulea it. - S. distique fr.

Monti di Giaveuo (Re in Bertol.).

Poa seslerioides All. fu confusa con la disticha dall'Allioni (Auct. pag. 40). Essa però se non vuolsi considerare come specie distinta (Sesleria pedemontana Reut.) è senza dubbio una varietà ( $\mathrm{S}$. disticha Pers. $\beta$ pedemontana Parlat.). Ha le foglie del culmo piane e la radice stolonifera; nella specie tutte le foglie sono filiformi, e la radice non è stolonifera.

Il Compendio delle Fl. it. del Cesati, etc. non fa cenno della località Giavenese notata dall' Allioni (Auct.) e dal nostro autore. nè dei monti del Pellice e del Chisone ove la rinvenne il dott. Rostan (vedi Guida alle Alpi Cozie, ecc., Pinerolo, Tip. Chiantore, 1879, pag. 104).

\section{Gen. 36. - Fo weria Pers.}

* Glumetta inferiore mutica o quasi.

K. cristata PERs. (Poa cristata Re-Willd). - Volgare nei prati e nei pascoli.

Per. Giugno, Luglio.

Paleo argentino gentile it. - Keulérie à crêtes fr.

Nel testo Poa cristat ${ }^{\mathrm{a}}$ Lin. 
* K. valesiaca GAud. - Su la Brunetta (Negri). Cenisio (Bouvier).

Per. Maggio.

K. sétacée fr.

K. setacea Pers. ¿. glabra Gren e Godr.

- Glumetta inferiore aristata.

ih. phleoides Pers. (Festuca phleoides Re-Vill.). Pascoli sterili e secchi.

Ann. Maggio, Giugno.

Forasacco comune it. - K. phléole fr.

Gen. 37. - Mlolimia Schrad.

M. coerulea Moencr. (Melica coerulea Re-Lin. Mant.). - In tutti i pascoli umidi montani.

Per. Luglio, Agosto.

Gramigna liscia it. -- Molinie blene fr.

M. serotina Mert. e Koch. (Agrostis serotina Re-Lin. Mant.). - Siti caldi a San Giorgio ed a Condove nella contrada detta la Roccea.

Per. Luglio, Agosto.

Paleo tardivo it. - M. tardive fr.

Diplachne serotina Link. Parlat.

Gen. 38. - Cymosurus Kunth.

C. cristatus Lin. - Prati montani e submontani. Per. Giugno, Luglio.

Gramigna canajuola it. - Cynosure à crêtes fr.

C. echinatus LrN. - Nei campi di segale montani. Ann. Maggio.

Ventolana it. - C. hérissé fr. 
Gen. 39. - Selerochlor Palis de Beauv.

S. dura Palis de Beauv. (Cynosurus durus Re-Lin.). - Presso Susa lungo la via fuori Porta de Lard. Ann. Maggío, Giugno.

Fienarola dura it. - Sclérochloa dure fr.

S. rigida Link. (Poa rigida Re-Lin.). - Nei pascoli sterili e caldi.

Ann. Giugno, Agosto.

Fienarola dei muri it. - S. roide fr.

GEN. 40, - Daetylis Lin.

D. glomerata LIN. - In tutti i prati.

Per. Giugno, Luglio.

Erba mazzolina it. - Dactyle aggloméré fr.

Gen. 41. - Mriza Lin.

B. media Lin. - Ovunque nei prati.

Per. Giugno, Luglio.

Tamburini, Tremolino it. - Brize intermédiaire fr.

Gen. 42. - Eraggrogtis Palis de Beauv.

E. megastachya LinK. (Briza eragrostis RE-LiN.). Frequentissima nei campi.

Ann. Giugno, Agosto.

Gramignone it. - Eragrostyde à longs épillets fr.

F. pilosa P. de Beaut. (Poa pilosa Re-Lin.). - Ne pascoli.

Ann. Luglio, Agosto.

Fienarola sottile it. - E. à manchettes fr. 
374

\section{Gen. 43. - Crlyeeria R. Brown.}

G. aquatica WAHLB. (Poa aquatica Re LiN.). - Intorno al lago di Caselette.

Per. Giugno, Luglio.

Fienarola acquatica it. - Glycèrie aquatique fr.

Offre buon pascolo alle pecore ed alle vacche.

G. Iluitans R. Brown. (Festuca fluitans RE-Lin.). Nei fossi e nelle acque fluenti.

Per. Giugno, Luglio.

Gramigna olivella it. - G. flottante fr.

Gen. 44. - Vulpia Gmel.

V. myuros GmeL. - (Festuca myurus Re-LiN.).

Ovunque nei pascoli sterili, ed ai margini dei campi.

Ann. Maggio, Giugno.

Paleo sottile it. - Vulpie queue-de-rat fr.

* V. ciliała Linז. - Musiné (Re, Fl. Tor. Festuca ciliata DC.).

Ann. Maggio.

Palèo barbuto it. - V. ciliée fr.

Gen. 45. - BBromans Parlat.

B. sterilis Lin. - Ovunque presso le case e nei pascoli.

Ann. Maggio, Luglio. (Culmo glabro).

Forasacco rosso it. -- Brome sterile fr.

B. tectorum Lin. - Abbonda nei siti aridi, caldi e declivi.

Ann. Maggio, Giugno. (Culmo pubescente).

Forasacco dei tetti it. - B. des toits fr. 
B. erectus Huds. (Bromus perennis Re VILL.). - Fu da me raccolta nei pascoli montani di Chianoc e sopra Monpantero quando percorsi questi luoghi in compagnia d' Ignazio Molineri.

Per. Maggio, Giugno. (Culmo pubescente).

Forasacco di corte barbe it. - B. dressé fr.

Volgare al Musiné (Re, Fl. Tor.)

È pascolo eccellente.

B. asper LiN. fil. - Intorno alle selve.

Per. Maggio, Giugno. (Culmo pubescente).

Ventolana ruvida it. - B. rude fr.

Gen. 46. - Serrofuleus Parlat.

S. squarrosus BAB. - (Bromus squarrosus RE. LIN.). - Ovunque nei prati aridi, declivi.

Ann. Maggio, Giugno.

Forasacco squarroso it. - Serrafalcus raboteux fr.

S. mollis ParLat. (Bromus mollis RE-LiN.). - Ann. Maggio, Giugno.

Forasacco peloso it. - S. mollet fr.

Gen. 4\%. - wen Lin.

"Specie perenni : glume 1-3 nervate: spighette non pendenti.

A. sempervirens Vill. - Nelle cave di gesso del Cenisio presso la Posta. Trovasi pure sopra le Ferrieres.

Per. Giugno, Luglio.

Vena zolfina it. - Avoine toujours verte fr.

Alpi di Bussolino nelle rupi presso il cosi detto Pian de Paris. 


\section{6}

* A. montana Vill. - Cenisio. (Parlat.). Cima dell'Assietta presso le antiche fortificazioni.

Per. Luglio, Agosto.

A. de montagne fr.

* A. Scheuchzeri All. - Cenisio alla G. Croce. (Bouvier). Musiné. (Re, Fl. Tor. sotto A. versicolor Vill.).

Per. Luglio, Agosto.

Vena bronzina it.

A. pratensis Lin. - Prati del Cenisio.

Per. Luǵlio, Agosto.

Vena pratajuola it. - A. des prés fr.

Musiné (Re, Fl. Tor.).

* A. pubescens Lin. - Cenisio. (Bonjean in Bertol.).

Per. Giugno, Luglio.

Vena pelosa it. - A. pubescente fr.

* A. amethystina DC. - Cenisio alla G. Croce. (Bouvier). Cenisio. (Parlat.).

Per. Maggio, Giugno.

A. améthyste fr.

* Specie annue: glume plurinervate : spighette pendenti.

A. sterilis Lin. - Nei campi.

Ann. Aprile, Maggio.

Vena selvatica it. - A. stérile fr.

* A. sativa Lin. - Coltivata.

Ann. Maggio, Giugno.

Avena it. - Biava vern. - A. cultivée fr.

Le avene in generale offrono un buon pascolo al bestiame. 
Gen. 48. - Tarimeram Parlat.

- Specie perenne: racemo a forma di spiga.

T. subspicatum P. de Bearvv. (Aira subspicata RE-Lin.). Nei prati più alti del Cenisio.

Per. Luglio, Agosto.

Avena dorata it. - Trisétum en épi fr.

- Specie perenni : pannocchia pit o meno aperta.

1) Resta uguale alla sua gluma.

T. flavescens P. DE Beauv. (Avena flavescens Re-Lin.). Volgare nei prati.

Per. Maggio, Luglio.

Gramigna bianca it. - T. jaunâtre fr.

Costituisce un ottimo pascolo.

T. disticophyllum P. DE Beauv. (Avena distycophyla RE-VıLL.). - Abbonda nei pascoli del Cenisio e segnatamente fra i macigni salendo alla cima di Ronche.

Per. Luglio, Agosto.

Gramigna screziata it. - T. à feuilles distiques fr.

Località medesime. (Negri).

2) Resta pith lunga della sua gluma.

* T. alpestre P. DE Beauv. - Alla Gran Croce sul Cenisio. (Bouvier, sotto Avena alpestris Host.).

Per. Luglio, Agosto.

Gramigna d'alpe it. - T. des alpes fr.

- Specie annua : pannocchia in forma di racemo cilindrico.

T. Gaudinianum Borss. (Avena Loefflingiana $R_{\mathrm{E}-\text { BaLBIS.). }}$ - Questa specie è stata trovata presso Foresto da Ignazio Molineri. Vedi Balbis, Miscel. bot., p. 13.

Anr. Aprile, Maggio.

T. negligée fr.

Su la Brunetta. (Negri). 
378

\section{Gen. 49. - Damenthomia DC.}

D. provincialis DC. (Avena spicata Re-BeLr.). - Siti mortani; frequente al Musiné.

Per. Maggio, Giugro.

Vena spigata it. - Darthonie de Provence fr.

Nel testo Avena spicata Lin., dal sinonimo di Bellardi.

D. decumbens DC. (Festuca decumbens Re-Lin.). Presso i fossi e le acque fluenti.

Per. Giugno, Luglio.

Gramigna logliarella it. - D. inclinée fr.

\section{Gen. 59. - Mage Lin.}

- Glumetta inferiore con cinque nervi appena visibili.

a) Linguetta (ligula) almeno in una fog'ia superiore allungata, raramente breve, e in tal caso, acuta, o arrotondita all' apice.

P. alpina Lin. - Frequente nelle Alpi.

* $\beta$ vivipara Parlat. (P. alpina vivipara Lin.). - Piccolo Cenisio (Bouvier), e altrove con la specie su le alpi.

${ }^{*} \gamma$ brevifolia Parlat. (Poa alpina $I^{a}$ brevifolia Gaud.). Piccolo Cenisio (Bouvier).

* o badensis Parlat. (Poa badensis Haenk.). - Cenisio, rupi di Patte creuse. (Chevalier in Bouvier).

Per. Giugno, Luglio.

Fienarola alpina it. - Paturin des alpes fr.

* P. coesia Smrte. - Cenisio ad Eau blanche. (Bouvier).

Per. Giugno, Luglio.

P. bleuâtre fr.

P. bulbosa Lin. - Ovunque nei pascoli declivi.

Per. Maggio, Giugno.

Fienarola scalogna it. - P. bulbeux fr. 
* P. concinna Gaud. $\beta$ Cenisia Bouvier. - Cenisio (Bouvier).

Per. Maggio, Giugño.

P. cenisia All. Auct. - Siti sassosi del Cenisio.

Per. Luglio, Agusto.

Fienarola serpeggiante it. - P. à feuilles distiques fr.

Poa distichophylla Gaud..

* P. Iaxa Haenk. - Alpi di Giaveno (Parlat.). Cima di Ronche (Negri).

Per. Luglio, Agosto.

Fienarola ciondola it. - P. élégant fr.

* P. minor Gaud. - Cenisio a Ronche (Bouvier).

Per. Luglio, Agosto.

P. mineur fr.

P. laxa (Balbis).

P. annua Lis. - Frequente intorno alle case, nelle vie e nei campi.

Ann. Aprile, Maggio.

Gramigna delle vie it. - P. annuel fr.

b) Linguetta (iigula) di tutte le foglie brevissima, troncata o quasi nulla, mai acuta, o arrotondita all' apice.

P. compressa LiN. - Abbonda nei campi e nei pascoli sassosi.

Per. Giugno, Luglio.

Ruba lana it. - P. comprimé fr.

P. nemoralis LiN. - Nelle selve.

Per. Giugno, Agosto.

Fienarola dei boschi it. - P. des bois fr.

Specie variabilissima e distinta dagli autori in moltissime sotto specie o varietà : A. vulgaris, B. firmula, C. rigidula, 
D. coartata, E. glauca, F. montana. (Parlat., Fl. it., V. 1, pag. 358. Gaudin synop. Fl. Helv., pag. 58-59. Comp. Fl. it., Cesati, ecc., pag. 70. Koch., Syn., ed. III, p. 698).

* Glumetta inferiore con 3-5 nervi vísibili e prominenti.

P. trivialis Lin. - Ovunque nei prati.

Per. Giugno, Luglio.

Granigna dei prati it. - P. commun fr.

P. sudetica Haenk. (Poa sylvatica Re-Vill.). - Nel Cenisio ai margini delle selve.

Per. Giugno, Luglio.

Fienarola grande it. - P. aplati fr.

P. pratensis Lin. - Ovunque nei prati.

$\beta$ angustifolia Sмıтн. (Poa angustifolia RE-LiN.) con la specie.

Per. Aprile, Maggio.

Fienarola it. - P. des prés fr.

Tutte le Poe offrono buon pascolo al bestiame, in ispecie la pratensis e la trivialis.

\section{GeN. 51. - Festuen LiN.}

1) Foglie, almeno le radicali, capillari o setacee.

- Foglie tutte capillari.

* F. violacea Gaud. - Cenisio a Ronche (Negri). Assietta alla cima.

Per. Luglio, Agosto.

Fetugue violette fr.

F. duriuscula Bertol. Comp. Fl. it., Cesati, etc. (Festuca duriuscula Re-Lin. in parte e Festuca amethystina RE-ALL. ?) - In tutti i pascoli e nei dumeti.

${ }^{\star} \beta$ cinerea (Glume pubescenti) F. cinerea BeLL. 
r ovina (Festuca ovina $R_{E}$-LIN.). (Culmi e foglie assai sottili, glumette aristate).

* ò tenuifolia (Culmi e foglie come nell'ovina: glumette mutiche) Festuca tenuifolia Pers.

${ }^{*} \varepsilon$ vivipara (Fiori trasformati in gemma). Festuca vivipara Wilid.

Tutte queste varictà coabitano con le specie tanto in pianura che su i monti.

Per. Maggio, Luglio.

Gramigna pavonazza it. - F. glauque fr.

- Foglie radicali capillari : quelle del culmo piane.

F. heterophylla Vilz. - Nelle selve montane.

Per. Maggio, Giugno.

Paleo delicato it. - F. à feuilles variées fr.

* F. nigrescens Lamk. - Cenisio a Ronche (Negri).

Per. Luglio, Agosto.

F. pumila Vill. - Trovata dal ch. Bellardi nei siti secchi del Cenisio.

Per. Luglio, Agosto.

Paleo pavonazzo it. - F. élégante $\mathrm{fr}$.

A Ronche (Negri, Bouvier).

F. flavescens Bell. - Frequente nel Cenisio e nei prati alpini di Chianoc.

Per. Luglio.

F. jaunâtre fr.

* F. varia Haenk. - Cenisio a Ronche (Negri) Cenisio su le roccie umide della montagna del Crin (Bouvier).

Per. Luglio, Agosto.

F. variable fr. 
* F. pilosa Haller fil. - Cenisio (Bouvier).

Per. Giugno, Luglio.

F. poilue fr.

2) Foglie piane pith o meno larghe.

F. spadicea Lin. (Poa Gerardi RE-ALL.). - In tutti i pascoli alpestri: frequente nelle alpi di Chianoc. Per. Giugno, Luglio.

Paleo dorato it. - F. dorée fr.

Sul Musiné (Re, FI. Tor.) prati del Colle della Portia (Re, in Bertol.). Cenisio a Ronche (Negri).

* F. sylvatica Vill. - Intorno a Bussolino (Bertero in Colla).

Per. Giugno, Luglio.

F. des bois fr.

Festuca altissima All. Auct. Balbis.

F. pratensis Huds. (Festuca elatior RE-Lin. Fl. suec.). - Nei prati pingui umidetti.

Per. Maggio, Giugno.

Paleo dei prati it. - F. des prés fr.

F. gigantea Vill. (Bromus giganteus Re Lin.). Presso i ruscelli nei siti ombrosi.

Per. Maggio, Giugno.

Ventolana gracile it. - F. élancée fr.

Le Festuche offrono buon pascolo al bestiame grosso e minuto. 
Sex. $4^{2}$ - Spighette a fiori ermafroditi distiate ala allece a fori masehili, o meutri mella stesar pampechis

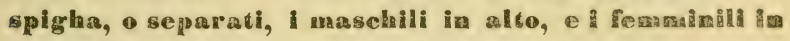
basso (Geu. Zea).

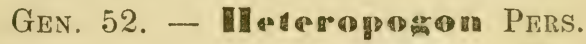

H. Allionii Rokm. e Schult. (Andropogon contorlum Re-Alz.). - Rupi calde di Monpantero e di Foresto. Per. Luglio, Agosto.

Barbone attorcigliato it. - Hétêropogon d'Allioni fr.

Nel testo Andropogon contortum Lin. dall'Allioni.

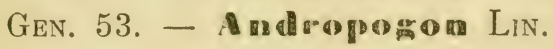

A. Ischaemum Lin. - Frequente nei luoghi sterili ed incolti.

Per. Luglio, Agosto.

Erba luciola it. - Barbon pied de poule fr.

\section{Gen. 54. - Cleryenpogen Trin.}

C. Grillus Trin. (Andropogon Grillus Re-Lin.). Qua e là nei prati macilenti e nei pascoli.

Per. Giugno, Luglio.

Erba da spazzole 1t. - Chrysopogon grillon fr.

GeN. 55. - Zege Lin.

Z. Mays Lin. - Coltivato: dell'America meridionale.

Ann. Giugno, Luglio.

Granturco it. - Melia vern. - Maïs cultivé fr.

L' introduzione del granturco in Europa data dalla scoperta d'America. 



\section{Classe III.}

\section{ACOTILEDONI VASCOLARI ${ }^{(*}$ (Acotyledoneae vascolares).}

A) Foglie (frondi) saldate insieme, e figuranti delle guaine dentate.

Ordine I. - Equisetacee (Equisetaceae $D C$ ). Gen. Equaisotum Lin.

* Cauli fertili e sterili difformi.

E. arvense Lin. - Equisetum minus o Cauda equina minor Off. - Frequente nei campi. Astringente.

Per. Marzo, Aprile. (Spiga ottusa).

Brusca, Coda di cavallo it. - Prêle des champs, Queue de rat fr.

* * Cauli fertili e sterili uniformi.

E. palustre LrN. - Nei prati umidi. Virtù del precedente.

Per. Giugno, Agosto. (Spiga ottusa).

Corregiola minore it. - P. des marais, queue de cheval fr.

(') Le piante acotiledoni cellulari della Fl. segusiensis non sono da noi riportate: esse dovrebbero per molte ragioni far parte di un volume separato. 
E. limosum Lin. - Intorno al lagº del Cenisio. $\beta$ ramosum (E. fluviatile RE-LIN.). - Equisetum majus - Cauda equina major. - Siti umidi presso i ruscelli. Virtù dei precedenti.

Per. Maggio, Luglio. (Spiga ottusa).

Fisalice it. - P. de bourbiers fr.

E. hyemale Lin. - Rasparella vern. - Lungo le acque nei siti sabbiosi e nei dumeti umidetti.

Per. Maggio, Luglio. (Spiga acuta mucronata!.

Asprella, Equiseto it. - P. d'hiver, P. des tourneurs fr.

Come rimedi gli equiseti non sono piu in uso.

B) Foglie distinte.

Ordine II. - Feldi (Fitices Juss.)

1) Sporangi sprovvisti di anello elastico, disposti in pannocchia nella parte superiore della foglia modificata.

Gen. 1. - Mpotryehinam Stwartz.

B. Iunaria Swartz. (Osmunda lunaria Re-Lin.). - Lunaria Off. - Nei prati montani ed alpini. Farmaco disusato.

Per. Giugno, Agosto.

Argentina it. - Botryche lunaire fr.

GEN. 2. - suatumato LIN.

0. regalis LIN. - Osmunda regalis o Filix florida Off. - Osmunda veru. - Nei monti di Giaveno.

Osmunda it. - Osmunde royale fr. 
2) Sporangi provvisti di anello elastico, disposti nella pagina inferiore delle foglie o sul margine delle stesse.

* Sori nudi cioè senza indusio, nè coperti dal margine reflesso della foglia.

\section{Gen. 3. - Ceterall Adans.}

G. officinarum WiLLD. (Asplenium ceterach RE-LiN.). - Asplenium o Ceterach Off. - Erba dorà vern. Frequente su le mura. Subastringente mucilaginosa.

Per. Aprile, Agosto.

Erba dorata, Cetracea it. - Ceterach, herbe à dorer fr.

Usata un giorno come rimedio nell'asma, nell'idrope, ecc., oggi del tutto dimenticata.

\section{Gen. 4. - Nothoelamea R. Brown.}

N. Marantae R. Brown. (Acrostichum Marantae RELiv.). - Volgarissima nelle rupi di Condove e specialmente nel sito detto la Grangietta.

Per. Aprile, Maggio.

Acrostico cetracea it. - Nothoclène de maranta fr.

Nelle rupi della Sagra di S. Michele scendendo ai laghi di Avigliana. (De Filippi).

Gen. 5. - Palypodium Lin.

P. vulgare Lin. - Polypodium o Polypodium quercinum Off. - Frequente nelle selve montane e ai piedi degli alberi. Radice dolce espettorante eccoprotica.

Per. Gitigno, Agosto.

Felce delle quercie it. - Argalizia servaia vern. - Polypode commun, Polypode de chêne fr.

Disusato in farmacia. 
P. Rethicum Lin. (Polypodium mollo RE-AlL.). Siti ombrosi del Cenisio.

Per. Giugno, Luglio.

Aspidio alpestre it. - P. des Grisons fr.

P. Phegopteris LiN. - Nei monti e specialmente presso la piana di S. Niccolà.

Ann. Giugno, Luglio.

Polipodio fagino it. - P. cilié fr.

P. Dryopteris Lin. - Nei siti montani ombrosi, sassosi e umidetti.

Per. Giugno, Agosto.

Driopeteri it. - P. dryoptère fr.

Alle Blaccie ed a Gravere (Negri).

* * Sori provvisti d'indusio.

Gen. 6. - Aspidilum Swartz.

A. Lonchitis Swartz. (Polypodium Lonchitis Re-LiN.). - Frequente nel Cenisio e sul bosco nero.

Per. Luglio, Agosto.

Lonchite it. - Aspidie en cimeterre $\mathrm{fr}$.

A. aculeatum Swartz. (Polypodium aculeatum ReLiN.). - Volgare nelle selve ombrose.

Per. Giugno, Agosto.

Felce maschia minore it. - Aspidic à cils roides fr.

Gen. \%. - Polysticum Roth.

(Rephrodium Mrchx)

P. Oreopteris DC. (Polypodium limbospermum REAlr.). - Frequenti nei castagneti di Giaveno e lungo il Sangone.

Ann. Luglio.

Aspidio montano it. - Polystic glanduleux fr. 
P. Thelypteris Rотн. (Polypodium Thelipteris RE-LIN. Mant.). - Intorno ai laghi di Avigliana.

Per. Giugno, Agosto.

Aspidio palustre it. - P. à bords roulés fr.

P. filix-mas Roтн. (Polypodium filix-mas RE-LiN. Filix-mas 0ff. - Nelle selve e nei siti ombrosi. Radice antielmintica, e raccomandata specialmente contro la tenia.

Per. Giugno, Agosto.

Felce maschia it. - P. fougère mâle fr.

Il rizoma di questa specie di felce è antico ed eccellente rimedio vermifugo in generale ed in particolare contro la tenia larga. (Cantani, Nothnagel).

P. cristatum Roth. (Polypodium crislatum Re-LiN.). Nel Cenisio.

Per. Luglio, Agosto.

P. ̀̀ crêtes fr.

P. rigidum DC. (Polypodium Villarii RE-BELI.). Siti sassosi nella piana di S. Niccolà.

Per. Luglio, Agosto.

Aspidio rigido it. - $\mathrm{P}$. roide $\mathrm{fr}$.

Gen. 8. - Cystopteris Bernh.

G. fragilis Bernh. (Polypodium fragile RE-LIN.). Presso le mura e fra i macigni.

Per. Giugno, Agosto.

Felce delicata it. - Cystoptère fragile fr.

Altipiano del Cenisio (Negri). 
c. alpina Link. (Polypodium alpinum RE-WLLF, ). Nelle rupi più alte del Cenisio.

Per. Giugno, Agosto.

Felce sottile it. - C. des alpes fr.

Nel testo Polyp. alpinum. Lamk. dall'Allioni.

C. montana Link. (Polypodium montanum RE-ALL.). - A Sestrières.

Per. Luglio, Agosto.

Felce cicutaria it. - C. de montagne fr.

Altipiano del Cenisio. (Negri). (Bonjean in Bertol.).

C. regia Косн. (Polypodium regium RE-LiN.). Presso le fonti tra Giaveno e Coazze.

Per. Giugno, Agosto.

Felce delicata it.

GEN. 9. - spleniam LIN.

* Foglie (frondi) pennate o bi-tripennate con segmenti pit o meno numerosi.

A. filix foemina Schrad. (Polypodium filix foemina RELin.). - Filix foemina Off. - Nei siti freddi umidetti montani ed alpini. Farmaco disusato.

Per. Giugno, Agosto.

Felce femina it. - Doradille fougère femelle fr.

A. Halleri DC. (Polypodium fontanum RE-LIN.). Volgare intorno Susa, e nella contrada di Fossimagna nei siti ombrosi ed umidi.

Per. Giugno, Agosto.

Felce dei fonti it. - D. de Haller fr. 
A. trichomanes Lis. - Polytrichum, Adianthum rubrum o Trichomanes Off. - Frequente nelle mura. Subastringente.

Per. Maggio, Agosto.

Tricomane it. - D. polytric fr.

Nel testo P. trichomanoides dall'Allioni.

A. viride Swartz. (Asplenium viride Re-Bell.) Siti ombrosi e sassosi del Cenisio e dei monti di Giaveno.

Per. Maggio, Agosto.

Tricomane verde it. - D. verte fr.

A. ruta muraria LiN. - Rula muraria o Paronychia Off. - Ovunque nelle mura. Farmaco disusato.

Per. Maggio, Agosto.

Ruta muraria it. - D. rue des murailles fr.

A. adianthum nigrum LIN. - Adianthum nigrum Off. - Nei siti sassosi montani.

Per. Maggio, Agosto.

Adianto nero it. - D. noire fr.

A. germanicum Weiss. (Asplenium alternifolium REWulf., Murr. Syst.). - Nei monti di Giaveno.

Per. Giugno, Agosto.

Paronichia bislunga it. - D. Germanique fr.

Nel testo A. alternifolium Lin.

* Foglie bi-tripartite con 2-5 segmenti.

A. septentrionale Swartz. (Acrostichum septentrionale Re-Lin.). - Nei siti ombrosi e nelle fenditure delle rupi.

er. Giugno, Agosto.

Falcetta sassatile it. - D. septentrionale fr. 
392

* Gen. 10. - Seolopemalliummer Swartz.

S. officinarum Swartz. - Sul Musiné (Re, Fl. Tor.)

Per. Giugno, Agosto.

Lingua cervina it. - Scolopendre officinale tr.

\section{GeN. 11. - Pleclingerin Roth.}

B. spicant Rotн. (Osmunda spicant Re-LiN.). - Nei castagneti elevati.

Per. Giugno, Agosto.

Lonchite minore it.

* * Sori coperti dal margine assottigliato e reflesso delle foglie.

Gen. 12. - Perois Swartz.

P. aquilina Lin. - Frequentissima nei pascoli montani e nei castagneti. Antielmintica.

Per. Giugno, Agosto.

Felce, felce grande it. - Ptéris aigle impérial fr.

\section{Gen. 13. - diamthum LIN.}

\section{A. capillus Veneris Lrn. - Capillus Veneris 0 ff.}

- Capelvenere vern. - Volgare nei siti umidi e sassosi tra Foresto e Bussolino, come alle Blaccie e Monpantero.

Per. Giugno, Luglio.

Capelvenere it. - Capillaire cheveux de Vénus fr.

Tenuto una volta come rimedio capace di promuovere le mestruazioni, oggi poco usato. 


\section{Gen. 14. - Allosorems Bernh.}

A. Crispus Bernh. (Osmunda Crispa Re-LiN.). Trovato da Ignazio Molineri nei luoghi sassosi dei monti di Giaveno e nel Cenisio sopra la Valle della Novalesa.

Per. Luglio, Agosto.

Felce coriandolina it.

\section{Gen. 15. - Chreilamthes Swartz.}

Ch. Odora Swartz. (Pteris acrosticha Re-Balbis). Frequente nelle mura delle vigne di Monpantero e di Croale.

Per. Maggio, Giugno.

Adianto odoroso it. - Cheilanthe odorant fr.

\section{GEN. 16. - Struthiopteris WILLD.}

S. germanica WiLld. (Osmunda siruthiopteris RE-LIN.). - Presso le acque nei siti ombrosi.

Per. Giugno, Luglio.

Felce penna di struzzo it.

Ordine III - Licopodiacee (Lycopodiaceae Rich.)

\section{Gen. 1. - Lyeopodium LIN.}

* Sporangi all'ascella di brattee simili alle foglio non disposti a spiga.

L. selago LiN. - Siti ombrosi alpestri.

Per. Luglio, Agosto.

Licopodio abietino it. -- Lycopode sélagine fr.

Cenisio (Bonjean in Bertol.). 
* Sporangi all'ascella di brattee diverse dalle foglie disposti a 2-6 spighe e portati da un lungo peduncolo.

L. clavatum Liv. - Braje d'ours vern. - Nei monti di Giaveno.

Per. Giugno, Agosto.

Musco clavato Lin. it. - L. à massue fr.

L. complanatum Lin. - Nei monti di Giaveno Per. Giugno, Agosto.

Licopodio spianato it. - L. apiati fr.

* + Spighe sessili solitarie all' apice dei rami: brattee diverse dalle foglie.

L. alpinum LiN. - Nei pascoli del Cenisio.

Per. Giugno, Agosto.

Licopodio alpino it. - L. des alpes fr.

L. annotinum Lis. -- Nei pascoli del Cenisio. Per. Giugno, Agosto.

Licopodio gineprino it. - L. à feuilles de genévrier fr.

\section{Gen. 2. - Sellaginella Spring.}

- Spighe peduncolate.

S. Helvetica Spring. (Lycopodium helveticum Re-Lin.). - Frequentissima nei pascoli dei monti e dei rolli.

Per. Aprile, Maggio.

Licopodio lenticolare it. - Sélagine de Suisse fr.

* "Spighe sessili, o quasi.

S. spinulosa A. Brown. (Lycopodium selaginoide RELiN.). - Siti umidi delle Alpi.

Per. Giugno, Agosto. (Foglie e brattee spinolose).

Licopodio spinuloso it. $-\mathrm{S}$. denticulée fr. 


\section{INDICE ALFABETICO}

\section{DEGLI ORDINI E DEI GENERI}

Acer Lin.

70 Amarantus Lin.

287

Acerrinee.

70

Achillaea Lin.

Anarillidee.

330

197

12

Ambrosiaces

221

Aconitum Lin.

A mididalee

105

13

Adenostyles Cass.

A mpatidacee

72

173

392

Adianthum Lin.

Ampelopsis Mich. . . 72

Amygdalus Lin. . . . 105

Adoxa Lin. . . . . 159

Aegilops Lin.

Aegopodium Lin.

367

145

Aesculus Lin.

71

Aethionema R. Brown

Anacamptis Rich.

324

Anagallis Lin.

282

Andropogon Lin. . . 383

Androsace Lin. . . . 280

Aethusa Lin.

Anemone Lin.

Angelica Lin. . . . 149

153

2,32

Anthemis Lin.

201

Anthoxanthum Lin. . . 364

119 Anthriscus Hoff. . . . 156

367 Anthyllis Lin. . . . 84

360 atiramee . . . 248

370 Antirrhinum Lin. . . 248

265 Apium Lin. . . . 145

120 pocines . . . . 233

315 Aquilegia Lin. . . , 10

315 Arabis Lin. . . . . 19

335 aragliacee . . . . 158

393 Arctostaphylos Adans. . 229

310 Arenaria Lin. . . . . 59

359 Aristolochia Lin. . . 296

126 sistolochiacee . . 296

56 Armeria Wild. . . . 284

56 Arnica Lin. . . . . 196

66 roidee... . . . 319

30 Aronia Pers. . . . 122

287. Aronicum Necker . . J95 
Arrhenatherum P. B. . Artemisia Lin. Arum Lin

Aselepiadee. Asparagacee Asparagus Lin. Asperugo Lin. Asperula Lin. Asphodelus Lin. Aspidium Swartz. Asplenium Lin. . Aster Lin.

Asterolinum Link. . Astragalus Lin.

Astrantia Lin. Athamanta Koch. . Atragene Lin. Atriplex Lin. Avena Lin. Azalea Lin. Ballota Lin.

\section{Halsaminee.}

Barbarea R. Brown Bartsia Lin. Bellidiastrum Cass. Bellis Lin.

Berardia Vill.

\section{IBerberidee .}

Berberis Lin. Betonica Lin.

\section{Hetulinee}

Betula Lin.

Bidens Lin.

Biscutella Lin.

Blechnu:is Roth

Blismus Panz.

Blitum Tourfn.

\section{Borraginee.}

Borrago Lin.

Botrychium Swartz. Brachypodium P. B. Brassica Lin.

Braya Sternb e Hopp. Briza Lin. Bromus Lin.
Pag.

Pag.

366

178

319

233

331

331

240

162

338

388

390

200

283

95

143

154

375

230

274

75

19

261

200

201

180

14

14

274

390

309

175

34

392

345

287

259

241

386

366

27

26

373 374

Brunella Lin.

276

Bryonia Lin. . . . . 128

Buffonia Lin. . . . . 56

Bulbocodium Lin. . . 340

Bunias Lin. . . . . 39

Buphthalmum Lin. . . 196

Bupleurum Lin. . . . 143

Butomacee . . . . . 315

Butomus Lin. . . . . 315

Buxus Lin. . . . . 300

Calamintha Bentham. . 269

Calamogrostis Adans. . 360

Calepina Desv. . . . . 38

Callitrichimee . . . 126

Callitriche Lin. . . . 126

230

10

32

223

221

301

Caprifogliacee. . . 159

Capsella Medik. . . 36

Cardamine Lin. . . . 22

Carduus Lin. . . . . 189

Carex Lin. . . . . 349

Carlina Lin. . . . . 185

Carpinus Lin. . . . . 304

Carum Lin. . . . . 146

Castanea Tourfn. . . 303

Caucalis Lin. . . . . . 155

Celastrinee. . . . . 78

Celtis Lin. . . . . 302

Centaurea Lin. . . . 182

Centhranthus DC. . . 168

Cephalanthera Rich. . 326

Cephalaria Schrad. . . 170

Cerastium Lin. . . . 62

Ceratofillacee . . . . 127

Ceratophyllum Lin. . . 127

Cercis Lin. . . . . . 104

Cerinthe Lin. . . . 243

387 
Pag.

Chaerophyllum Lin. . . 157

Chamaeorchis Rich.

325

Cheilanthes Swartz

393

Chelidonium Tourfn.

Chenopodiacee.

Chenopodium A. Meyer. 288

Cherleria Lin.

Chlora Lin.

56

Chondrilla L

234

Chrysopogon Trin.

Chrysosplenium Lin.

Cicer Lin. .

Cichorium Lin.

\section{Ciperacee}

210

383

141

Crocifere

Pag.

Circaea Lin

Cirsium Tournf.

98

204

314

125

186

Cistinee

Cistus Lin.

Cladium R. Brow.

Clematis Lin.

Crocus Lin. . . . . 329

Cucubalus Lin. . . . . 51

Cucumis Lin. . . . 129

Cucurbita Lin. . . . 328

Cucurbitacee . . . 128

Cupolifere . . . . 303

Cuscuta Lin. . . . . 238

Cyclamen Lin. . . . 280

233

189

372

358

239

344

81

389

294

373

378

32 Datura Lin. . . . 245

323 Daucus Lin. . . . . . 149

339 Delphinium Lin. . . 12

339 Dentaria Lin. . . . 24

92 Deschampsia P. B. . . 369

115 Deyeuxia P. B. . . . . 359

172 Dianthus Lin. . . . 48

310 Dictamnus Lin.. . . 77

156 Digitalis Lin. . . . 250

332 Digitaria Scop. . . . 365

238 Diplotaxis DC. . . . 28

238 dipsacee . . . . 170

328 Dipsacus Lin. . . . . 170

159 Doronicum Lin. . . . 195

159 Doryenium Tournf . . 91

97 Draba Lin. . . . . 31

279 Dracocephalum Lin. . . 268

16 Drosera Lin. . . . . 46

304 Droseracee . . . . 46

121 Dryas Lin. . . . . . 110

133 Echinaria Desf. . . . 368

132 Echinops Lin. . . . 185

Crataegus Lin. . . . 121
Crepis Lin. . . . . 213 Echinospermum Swartz . 239

Crataegus Lin. . . . 121
Crepis Lin. . . . . 213 Echinospermum Swartz 239
Echium Lin. . . . . 242 
Eleagnee

Eleocharis R. Brown.

Elyna Schrad.

Empetracee

Empetrum Tourn.

Ephedra Lin.

Epilobium Lin. .

Epipactis Rich.

Eugrisetacee

Ericinee.

Equisetum Lin.

Eragrostis P. B.

Erigeron Lin.

Eriophorum Lin.

Eritrichium Schrad.

Erodium L'Her

Eruca DC.

Erucastrum Schimper e Spen. . . . .

Ervum Lin. .

Eryngium Lin.

Erysimum Lin.

Erythraea Rich.

Erytronium Lin.

Eupatorium Lin.

Euphorbia Lin. .

Euforbiace

Euphrasia Lin.

Evonymus Lin.

Faba Tournf.

Fagus Lin.

\section{Telei}

Festuca Lin.

Ficus Lin.

Filago Lin.

Foeniculum Hoff.

Fragaria Lin.

Fraxinus Lin.

Fumaria Lin.

Fumariacee

Gagea Salisb.

Galega Lin. .

Galeopsis Lin.

Galium Lin. .

Gaya Gaud.

\section{Pag}

295

346

348

296

296

310

123

327

385

229

$38 \pi$

373

199

348

240

75

29

28

101

142

26

237

333

172

297

279

262

78

101

303

386

380

302

176

152

115

233

17

16

334

92

273

163

153
Genista Lin.

Gentiana Lin.

Grenzianace

Gereraniacee

Geranium Lin. . . . . 72

Geum Lin. . . . . 110

Gigliacee

Givncacee

Giuncaginacee

Gladiolus Lin.

Globularia Lin.

folobulariacee.

Glyceria R. Brown.

Gnaphalium Lin.

Goodyera R. Brown.

\section{Ceranimacee}

Gratiola Lin.

Gregoria Duby.

333

341

316

329

283

283

374

176

328

357

251

279

Gymnadenia R. Brown . 322

Gypsophila Lin.

50

Cúpossalariee . . . . 136

Hedera Lin. . . . . 158

Helianthemum Tournf. . 40

Heliotropium Iin. . . 243

Hellcborus Lin.

10

Helminthia Juss. . . . 206

Heracleum Lin. . . . 150

Herminium R. Brown. . 325

Herniaria Lin. . . . 150

Hesperis Lin. . . . . 24

Heteropogon Pers. . . 333

Hieracium Lin. . . . 216

Hippocrepis Lin. . . . 93

Hippophae Lin. . . . 295

Hippuris Lin. . . . . \$26

Holcus Lin. . . . . 364

Holosteum Lin.

Homogyne Cass.

59

Hordeum Lin.

174

358

Hugueninia Reichnb. . 26

Humulus Lin. . . . 301

Hutchinsia R. Brown. . 36

Hydrocotyle Lin. . . . 142

Hyoscyamus Lin. . . 245

Hypericum Lin. 
Pag. Hyssopus Lin. Iberis Lin. Ilex Lin.

Impatiens Lin. Imperatoria Lin. Inula Lin.

\section{Ipericinase}

\section{耳ppocastanee}

Eppuridee

\section{面idiee.}

Iris Parl.

Isatis Lin.

Isnardia Lin.

Jasione Lin.

\section{Duglamalec}

Juglans Lin.

Juncus Lin.

Juniperus Lin.

Kentrophyllum Neck.

Knautia Coult.

Kobresia Willd.

Koeleria Pers.

Labiate

Lactuca Lin.

Lamium Lin.

Lappa Tournf.

Lapsana Lin.

Laserpitium Lin.

Lasiagrostis Link.

Lathraea Lin.

Lathyrus Lin.

Lavandula Lin.

Lemna Lin.

Eemanacee

Leatibaมี่อ

Leontodon Lin.

Leonurus Lin.

Lepidium Lin.

Leucanthemum Tournf. . 202

Leucoium Lin.

Leuzea DC.

Libanotis Crantz.

\section{Lieopodiacee}

Ligustrum Lin.
209

271

34

232

75

149

193

68

71

126

329

329

37

125

221

303

303

343

311

190

171

349

371

263

211

272

IS1

204

147

360

257

102

268

318

318

¿77

205

272

35

330

181

153

393

232
Liliastrum Tournf.

Lilium Lin.

Limodorum Swartz.

Linee

Linaria Juss.

Linosyris DC.

Linum Lin.

Listera R. Brown.

Lithospermum Lin.

Eitrar'ie

Lloydia Salisb.

Lolium Lin.

Lonicera Lin.

Lotus Lin.

Lunaria Lin.

Luzula DC.

Lychnis Lin.

Lycopodium Lin.

Lycopsis Lin.

Lycopus Lin.

Lysimachia Lin.

Lythrum Lin.

Malachium Fries.

Malva Lin.

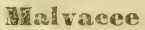

Marrubium Lin.

Matricaria Lin.. . . 201

Matthiola R. Brown. . . 18

Mayanthemum Wigg. . 332

Medicago Lin.

85

Melampyrum Lin. . . 258

Melica Lin.

369

Melilotus Tournf. . . 86

Melissa Lin.

270

Melittis Lin.

275

Mentha Lin. . . . . 266

Menyanthes Lin. . . 234

Mercurialis Lin. . . . 300

Mespilus Lin. . . . 122

Meum Tournf. . . . 152

Micropus Lin. . . . 174

Milium Lin. . . . . 362

Moenchia Ehrh. . . 62

Moerhingia Lin. . . . 60

Molinia Schrad. . : 372 


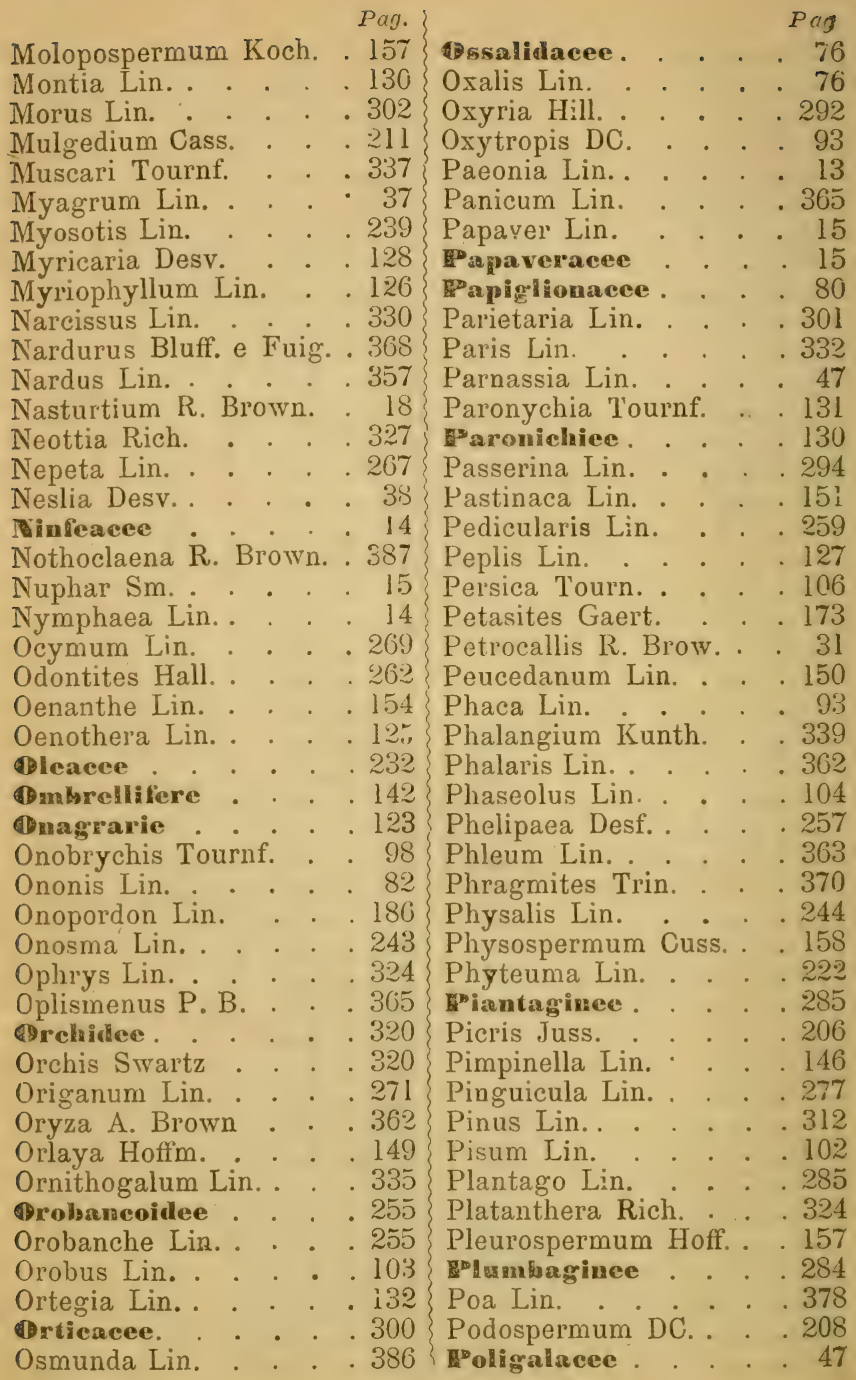


Polycarpon Lin.

Polycnemum Lin.

Polygala Lin.

Polygonatum Tournf. .

Polygonum Lin.

Polypodium Lin.

Polysticum Roth.

\section{Eomifere}

Populus Lin.

Portulaca Lin.

\section{다욤olacee}

Fotamacee

Potamogeton Lin.

Potentilla Lin.

Poterium Lin.

Prenanthes Lin.

Primula Lin.

\section{Erimulacee.}

Prunus Tournf.

Psilurus Trin.

Pteris Swartz.

Ptychotis Koch. .

Pulicaria Gaert. .

Pulmonaria Lin.

Pyrethrum Sm. .

Pyrola Tournf.

Fyrolacee.

Pyrus Lin.

Quercus Lin. .

Homnacee

Fihanunculacee

Ranunculus Lin.

Raphanus Lin.

Rapistrum Boerh.

Reseda Lin.

IFesedlacee

Rhamnus Lin.

Rhinanthus Lin.

Rhodiola Lin. . . . . . 132

Rhododendron Lin. . . 230

Rhus Lin.

Rhynchospora Vahl.

Ribes Lin.

Ftimantacee
Pag.

132

287

47

331

292

387

388

121

308

129

129

316

316

111

121

210

278

278

106

358

392

147

194

242

203

230

$2 ; 30$

122

304

79

1

5

39

39

45

45

79

261

78

347

136

258
Ricchia Parl.

Robinia DC.

Rosa Lin.

IBesacee

Rosmarinus Tournf $\quad 264$

Prubiacee .

Rubus Lin.

Rumex Lin.

Ruscus Lin.

Ruta Lin.

metrucee

Sagina Lin.

Sallicinee

Salix Lin.

Salvia Lin.

Sambucus Lin.

Samolus Lin.

Sanguisorba Lin.

Sanicula Lin. .

Santalacee

Santolina Lin

Saponaria Lin.

Satureia Lin. .

Saussurea DC.

Sassifragee.

Saxifraga Lin.

Scabiosa Lin.

Scandix Lin.

Scheuchzeria Lin.

Schoenus Lin.

Scilla Lin.

Scirpus Lin.

Silerantee

Scleranthus Lin.

Sclerochloa P. B.

Scolopendrium Swartz. . 392

Scorzonera Lin. .

Scrophularia Lin.

Scutellaria Lin. .

Secale Lin

Sedum DC.

Selaginella Spring.

Selinum Lin.

Sempervivum Lin.

Senebiera Pers.
Pag.

323

92

117

109

162

116

290

332

77

77

58

305

305

263

160)

282

120

143

295

175

50

269

180

137

137

171

155

316

345

335

346

132

132

373

208

247

375

366

133

394

149

135

38 


\section{Silenee}

Silene Lin.

Sinapis Lin.

Sison Lin.

Sisymbrium Lin. Sium Lin.

\section{Solanacee}

Solanum Lin.

Soldanella Lin.

Solidago Lin. .

Sonchus Lin. .

Sorbus Lin.

Sparganium Tournf.

Specularia Heister.

Spergula Lin.

Spergularia Pers.

Spinacia Lin.

Spiraea Lin.

Spiranthes Rich.

Spirodela Schleid.

Stachys Lin

Stellaria Lin.

Stenactis Cassin.

Stipa Lin.

Streptopus Rich.

Struthiopteris Willd.

Tetragonolobus Scop.

Teucrium Lin.

Thalictrum Lin.

Theligonum Lin.

Thesium Lin.

264

Thlaspi Lin.

5

Thuincia Roth.

Thymus Lin.

\section{Tifacee}

33

Tigliaces.

Tilia Lin. .

Tommasinia Bertol.

Tordylium Lin.

Triticum Lin.

Succisa Mertens et Koch

Swertia Lin.

331

Tulipa Lin.

$33: 9$

Tunica Scop. .

Turgenia Hoffm.

Symphitum Lin.

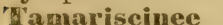

Tussilago Lin.

$19: 3$

Typha Lin.

318

Ulmus Lin. . . . . . . 302

Urtica Lin. . . . . 300

Utricularia Lin. . . . . 277

Eaccince. . . . . . 228

128 Vaccinium Lin... . . 228

Tamus Lin.

Tanacetum Lin.

333

175

Taraxacum Juss.

Taxus Lin.

210

Valeriana Lin.

Valerianella Pollich. 
$4(13$

Pag. \{

Pag.

Verbascum Lin... . . 245 Vitis Lin.... . . 72

Verbena Lin. . . . 276 Vulpia Gmelin. . . . 374

Werbenacee . . . 276 Xanthium Lin. . . . 221

Veronica Lin. . . . . 251 Xeranthemum Lin. . . 184

Vesicaria Lamk. . . . 29 Xiphion Parl. . . . 330

Viburnum Lin. . . . . 160 Zacyntha Tournf. . . . 211

Vicia Lin . . . . . 99 Zanichellia Lin. . . . 317

Vinca Lin. . . . . . 233 Zea Lin. . . . . . 383

Viola Lin. . . . . 42 Zigofllee. . . . 76

giolacee . . . . . 42$\}$

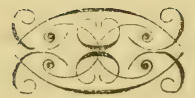





\section{ERRATA-CORRIGE}

Pag. III v. 18 ingiura

" XI " 14 cinqui

" 13,9 della quale

๑ 22,17 Ferrere

" 24 " 26 Arabis. Thaliana

" 29 "20 Gen. 18 - Vescicaria Lin.

> 29 "26 Vescicaire

" 40 "20-21 opposte stipulate, eccetto nell'H. Italicum.

^ 46 " 4 Ros. 48 \% 21 ( D. atrorubens RE-Alt. - Pascoli e pratimacilenti

" 53 "291876

" 57 " 26 (A. tenuifolia RELin.)

" 58 " 21 (Spergula saginoides Lin.)

> 61 20 Rossa

" 64 "21 Rocca

" 75 "22 teuchez

" $80 " 15$ Actragalus

" 110 " 14 Cametrio gentile

" 111 " 13 Voli-Chiapusso

" 139,14 Airoides

, 149 " 9 Carci

" 154 " 12 Calmonte

$" 175,22$ altielmintica leggi ingiuria

" cinque

" del quale

" Ferriere

" Arabis Thaliana

" Gen. 18 - Vesicaria Lin.

"Vesicaire

" opposte stipulate I senza stipule nell' $H$. Italicum).

$"$ Ros

" (D. Atrorubens REAlL. - Pascoli e prati macilenti.

, 1866

” (Aren. tenuifolia RELin.)

" Spergula saginoides Lin.

" Russa

" Roccea

" touchez

"Astragalus

" Camedrio alpino

" Chiapusso-Toli

" Aizoides

" Carvi

" Chiomonte

" antielmintica 
Pag. 178 v. 18 Brunetta pressn

" 199 " 24 ne

" 206 " 18 Pieris

" 219 " 15 Moliner

" 230 " 12 Gen. Rhododen. dron

" 262 " 1 Euphrasia Link.

" 267 " 12 Cultera

" 272,3 stami paralleli

" 279 " 10 D'Unmbert

" 313 " 12 P. sapin blanc

Ai generi $\left\{\begin{array}{l}\text { Erysimum } \\ \text { Ortegia } \\ \text { Aegopodium } \\ \text { Menyanthes } \\ \text { Heliotropium }\end{array}\right.$

Al genere Cerastium ed alle specie $\left\{\begin{array}{l}\beta \text { Bryoides } \\ \text { S. exarata }\end{array}\right.$

Alla pag. 296 dopo (Aristolochieae Juss.) si aggiunga in un nuovo capoverso il nome dell' unico genere: Aristolochia LiN.
" Brunetta e presso

$"$ nel

" Picris

" Molineri

" Gen. 4.- Rhododendron

" Euphrasia Lin.

" Cullera

" D) Stami paralleli

" D'Umbert

" Sapin blane

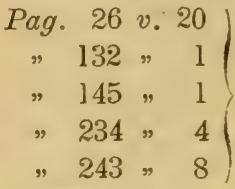

Si aggiunga LIN.

" $62 " 21$

" $140 " 13$ (l'asterisco
" 139,9$\}$ Si tolga 



Prezzo: I. 5 


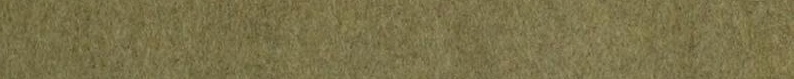
T.

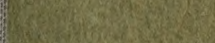

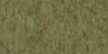

\subsection{0.} 3.

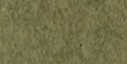

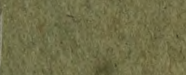
$\log _{5} \log ^{3}$
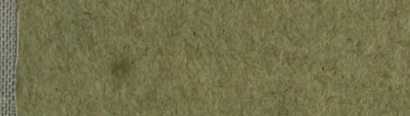

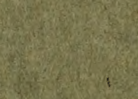
15.

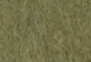

- 350 or

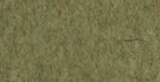

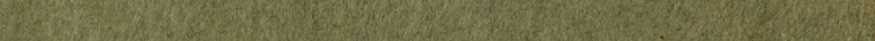
20. W.

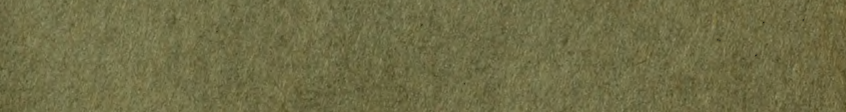
Q3. W. 
(3)

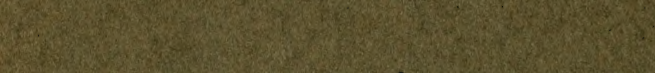

3.

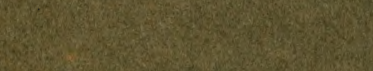

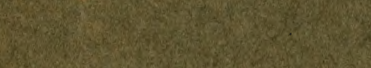

1.

(16)

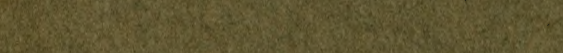

W.

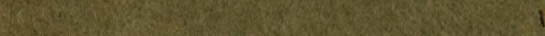

3.

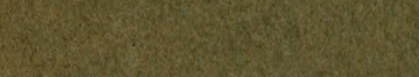

(5)

(6)

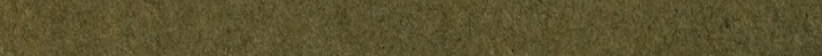


